\title{
MIMO Transceiver Architectures for Coded Communication with Limited CSI Feedback
}

by

\author{
Matthew Dorrance \\ A thesis submitted to \\ the Faculty of Graduate Studies and Research \\ in partial fulfilment of \\ the requirements for the degree of \\ Master of Applied Science in Electrical Engineering
}

Ottawa-Carleton Institute for

Electrical and Computer Engineering

Department of Systems and Computer Engineering

Carleton University

Ottawa, Ontario, Canada

September 29, 2008

Copyright @2008 - Matthew Dorrance 


$\begin{array}{ll}\begin{array}{l}\text { Library and } \\ \text { Archives Canada }\end{array} & \begin{array}{l}\text { Bibliothèque et } \\ \text { Archives Canada }\end{array} \\ \begin{array}{l}\text { Published Heritage } \\ \text { Branch }\end{array} & \begin{array}{l}\text { Direction du } \\ \text { Patrimoine de l'édition }\end{array} \\ \begin{array}{l}\text { 395 Wellington Street } \\ \text { Ottawa ON K1A 0N4 } \\ \text { Canada }\end{array} & \begin{array}{l}\text { 395, rue Wellington } \\ \text { Ottawa ON K1A 0N4 } \\ \text { Canada }\end{array}\end{array}$

Your file Votre référence ISBN: 978-0-494-44038-4 Our file Notre référence ISBN: 978-0-494-44038-4

NOTICE:

The author has granted a nonexclusive license allowing Library and Archives Canada to reproduce, publish, archive, preserve, conserve, communicate to the public by telecommunication or on the Internet, loan, distribute and sell theses worldwide, for commercial or noncommercial purposes, in microform, paper, electronic and/or any other formats.

The author retains copyright ownership and moral rights in this thesis. Neither the thesis nor substantial extracts from it may be printed or otherwise reproduced without the author's permission.
AVIS:

L'auteur a accordé une licence non exclusive permettant à la Bibliothèque et Archives Canada de reproduire, publier, archiver, sauvegarder, conserver, transmettre au public par télécommunication ou par l'Internet, prêter, distribuer et vendre des thèses partout dans le monde, à des fins commerciales ou autres, sur support microforme, papier, électronique et/ou autres formats.

L'auteur conserve la propriété du droit d'auteur et des droits moraux qui protège cette thèse. $\mathrm{Ni}$ la thèse ni des extraits substantiels de celle-ci ne doivent être imprimés ou autrement reproduits sans son autorisation.
In compliance with the Canadian Privacy Act some supporting forms may have been removed from this thesis.

While these forms may be included in the document page count, their removal does not represent any loss of content from the thesis.
Conformément à la loi canadienne sur la protection de la vie privée, quelques formulaires secondaires ont été enlevés de cette thèse.

Bien que ces formulaires aient inclus dans la pagination, il n'y aura aucun contenu manquant.

\section{Canada}




\section{Abstract}

Multiple-input and multiple-output (MIMO) wireless communication systems have been the focus of intense study for over a decade because they promise to deliver very high spectral efficiencies. Additionally, in recent years rate-compatible (RC) codes have drawn much attention because they are capable of approaching channel capacity for a small amount of system overhead. In this thesis we combine the RC Quasi-Cyclic (RC/QC) LDPC codes of the 3GPP2 standard with rate-constrained linear MIMO transceivers that have limited channel state information (CSI) feedback to implement $\mathrm{RC} / \mathrm{QC}-\mathrm{MIMO}$ communication systems. Out of the combination of these $\mathrm{RC} / \mathrm{QC}$ LDPC codes with a CSI-feedback-limited, rate-constrained MIMO system, evolves new implementation issues. To understand the implementation issues that face each $\mathrm{RC} / \mathrm{QC}-\mathrm{MIMO}$ transceiver we provide a comprehensive study of the performance of each linear transceiver described and implemented in this thesis. In attempting to solve the implementation issues that exist for a RC/QC-MIMO system we develop new signal processing algorithms including a new iterative water-filling algorithm for rate-constrained MIMO and new soft-symbol and log-likelihood ratio estimators that have low average computational complexity. Also, a Hybrid transceiver that allows RC/QC-MIMO to quickly converge on its maximum nominal rate, while achieving good overall throughput performance is proposed. Furthermore, because the MIMO system is assumed to have limited CSI feedback a parameterized quantization strategy is designed to quantize the parameters of and represent a semi-unitary matrix. The 
new algorithms and Hybrid transceiver coupled with the combination of $\mathrm{RC} / \mathrm{QC}$ LDPC codes and MIMO are the main contributions of this thesis. 
Firstly, I dedicate this thesis to my parents. Their patience and sacrifice were a great help in the writing process.

Secondly, I wish to dedicate this thesis to anyone who has ever dared to try and come out better for it. The process of writing this thesis was much like a marathon. Those who've ran one know that it is a two-tiered race - the first 20 miles, and the last 6 miles - where the last 6 miles require the most dedication, persistence and faith. 


\section{Acknowledgments}

First, I would like to thank my supervisor, Dr. Ian Marsland, for his guidance, teaching and friendship over the past couple of years. Dr. Marsland gave me the opportunity to research those problems that interested me, guiding me through the research process. He was a great help in writing and organizing this thesis, and taught me a great deal on how to express and formulate my ideas.

A great deal of thanks goes to my parents for their help, patience and sacrifice not only these past two years, but throughout my entire life. I am deeply indebted to them.

I am grateful to Dr. Shahram Yousefi for giving me the opportunity to research with him as an undergraduate student in the summer of 2006 . The research done that summer inspired the work in this thesis.

A thank you to my sisters, Erin and Kristin for their support and encouragement over all the years that I've known them. In addition, I would like to also thank my extended family (Hagan and Dorrance) for all their support.

To my lab-mates, mostly Furuzan Atay and Basak Can. I thank Furuzan for her friendship and providing me with a sounding board for many of my ideas and 
problems. I thank Basak for her friendship and for demonstrating to me how far belief and persistence can take you.

To all of my friends, but mostly Andrew Mackinder. Andrew has been an invaluable friend through this entire process, encouraging me and listening to some of my "high-in-the-sky" ideas.

A thank you goes to my girlfriend Isabelle Palad. Isabelle helped me get through those last 6 miles (see dedication) with my sanity intact, showing faith in me, as well as understanding these last three months.

I would also like to thank my defense committee of Dr. Claude D'Amours, Dr. Amir Banihashemi and Dr. Adrian Chan for their time and recommendations. 


\section{Table of Contents}

Abstract

Acknowledgments $\quad$ v

Table of Contents vii

List of Tables xiii

List of Figures xiv

List of Acronyms xix

List of Mathematical Operators and Symbols xxii

1 Introduction 1

1.1 Background and Motivation ................. 1

1.1.1 MIMO: The Metamorphosis . . . . . . . . . . . . . 1

1.1.2 Joint Transceiver Design: The Channel Decomposition Method 3

1.1.3 Achieving Throughput Close to Capacity: An Adaptable ErrorControl Code ....................... 4

1.1.4 A Combined Approach: A Low-Complexity MIMO Architecture for Achieving High Throughput . . . . . . . . . . 6

1.2 List of Contributions . . . . . . . . . . . . . . . . . . 7 
1.3 List of Publications Resulting from this Work . . . . . . . . 8

1.4 Thesis Outline . . . . . . . . . . . . . . . . . . . . . 8

\section{A Comprehensive Study of Channel Decomposition-} $\begin{array}{lr}\text { Based MIMO Transceivers } & 11\end{array}$

2 MIMO Systems $\quad 12$

2.1 MIMO System Model . . . . . . . . . . . . . . . . . . . . . . . 12

$2.1 .1 \quad$ Symbol Mapping . . . . . . . . . . . . . . . . . . . . . . 12

2.1 .2 Precoder . . . . . . . . . . . . . . . . . . 13

2.1.3 Discrete-time MIMO channel . . . . . . . . . . . . . . 14

$2.1 .4 \quad$ Spatial Filter . . . . . . . . . . . . . . . . 14

2.1 .5 Detector . . . . . . . . . . . . . . . . 15

2.1 .6 Decision Device . . . . . . . . . . . . . . . . . . 15

2.2 MIMO with an Uninformed Transmitter . . . . . . . . . . . 15

2.2.1 Maximum Likelihood Detector . . . . . . . . . . . . . 16

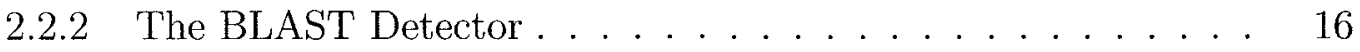

2.2.3 The QR Implementation of the BLAST Detector . . . . . 20

2.3 MIMO Transceivers with a Fully Informed Transmitter . . . . . . . 23

2.3.1 Capacity Achieving Design: Singular Value Decomposition with the Water-Filling Algorithm . . . . . . . . . . . 24

2.3.2 The Geometric Mean Decomposition: Equal Gain Subchannels 26

2.3.3 Antenna Selection Geometric Mean Decomposition . . . . . 28

2.3.4 Uniform Channel Decomposition: A Capacity Achieving Solution with Equal SINR Subchannels . . . . . . . . . . . 28

2.3 .5 GMD-MMSE . . . . . . . . . . . . . . . . 30 
3 Evaluating MIMO System Performance 32

3.1 MIMO Capacity . . . . . . . . . . . . . . . . . . . . . . . 32

3.1.1 Instantaneous Unconstrained Capacity of MIMO . . . . . . . 32

3.1.2 Ergodic Unconstrained Capacity of MIMO . . . . . . . . . . 34

3.2 Unconstrained Capacity of MIMO Transceivers . . . . . . . . 36

3.2.1 Unconstrained Capacity of UT Transceivers . . . . . . . 36

3.2.2 Unconstrained Capacity of IT Transceivers . . . . . . . . . . 37

3.3 BER performance of the Channel Decomposition Methods with Fixed

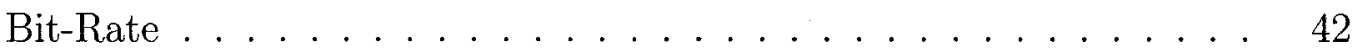

3.3.1 BER Performance of UT MIMO Systems . . . . . . . . . . 43

3.3.2 BER Performance of IT MIMO Systems $\ldots \ldots \ldots$

\section{Design of a Discrete Rate-Constrained Coded MIMO System}

4 Discrete Rate-Constrained MIMO System Design 53

4.1 System Model . . . . . . . . . . . . . . . . . . . . 53

4.1 .1 Encoder . . . . . . . . . . . . . . . . . 55

$4.1 .2 \quad$ Symbol Mapper . . . . . . . . . . . . . . . . . . 57

4.1 .3 Detector . . . . . . . . . . . . . . . . . 57

4.1 .4 Type-II H-ARQ . . . . . . . . . . . . . . . . . . . 64

4.1.5 Multiple Versus Single Codeword Demultiplexing of Data Stream 65

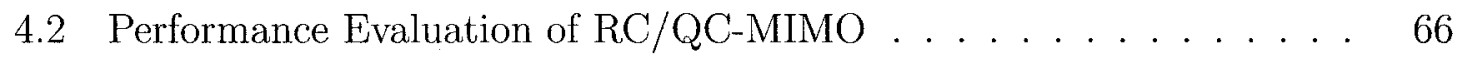

4.2.1 The Average Realizable Rate of RC/QC-MIMO . . . . . . 66

4.2.2 Experimental Performance of RC-QC LDPC Codes in an AWGN Channel . . . . . . . . . . . . . . . . . . . . . 68 
4.2.3 Theoretical Constrained Throughput Performance of MIMO Transceivers ....................... 71

4.2.4 Experimental Throughput Performance of RC/QC-MIMO . . 75

5 Power and Bit-Rate Allocation for the Rate-Constrained MIMO $\begin{array}{ll}\text { Subchannel } & 80\end{array}$

5.1 CWFA: Power and Bit-Rate Allocation Algorithms . . . . . . . 81

5.2 Rate-Constrained Antenna Selection Algorithm for ASGMD . . . . 87

5.3 Performance Evaluation of CWFAs . . . . . . . . . . . 87

5.3.1 Theoretical Throughput Performance of CWFAs . . . . . . . 87

5.3.2 Experimental Throughput Performance of RC/QC-MIMO with CWFA .......................... 93

6 Tunable Soft-Symbol Detection for Layered BLAST Detection $\quad 97$

6.1 The Fully-Tuned SS/LLRE . . . . . . . . . . . . . . . . . . . 98

6.2 Tunable SS/LLRE . . . . . . . . . . . . . . . . . . 101

6.3 BER Performance of the MMSE-ISF Detector with T-SS/LLRE . . . 103

6.4 Experimental Throughput Performance of RC/QC-MIMO with TSS/LLRE . . . . . . . . . . . . . . . . . 111

7 Compromising Between SVD and GMD: A Hybrid Transceiver 115

7.1 Hybrid Transceiver Design . . . . . . . . . . . . . . . . 115

7.2 Experimental Throughput Performance of the Hybrid Transceiver . . 118

\section{MIMO Transceiver Design for Systems with Partial CSI Feedback}


8.1 Classification of the Types of Quantizable Information and the Amount of Feedback Required by each MIMO Transceiver . . . . . . . . . . 124

8.1.1 Feedback Requirements of SVD-CWFA . . . . . . . . . . . . 124

8.1.2 Feedback Requirements of SVD . . . . . . . . . . . . . 125

8.1.3 Feedback Requirements of GMD . . . . . . . . . . . . 125

8.1.4 Feedback Requirements of ASGMD . . . . . . . . . 126

8.1.5 Feedback Requirements of UCD . . . . . . . . . . . 126

8.1.6 Feedback Requirements of Hybrid . . . . . . . . . . 127

8.1.7 Comparison of the Feedback Requirements of the Different MIMO Transceivers . . . . . . . . . . . . . . . . 127

8.2 The Impact of Quantization Errors and Detector Design for a PIT MIMO System . . . . . . . . . . . . . . . . . . . . 130

8.2.1 An Example of the Impact of Quantization Errors: PIT GMDSIC . . . . . . . . . . . . . . . . .

8.2.2 Equivalent Channel Matrix-Matched MMSE-SIC and MMSEISF Filters for PIT MIMO Transceivers . . . . . . . . . . . . 132

8.3 Quantization of a Semi-Unitary Matrix . . . . . . . . . . . 133

8.3.1 Characterizing a Semi-Unitary Matrix by a Set of Parameters 134

8.3.2 Parameter Quantization Strategy for a Semi-Unitary Precoder Matrix . . . . . . . . . . . . . . . 136

8.4 Uncoded BER Performance of PIT MIMO . . . . . . . . . . . 138

8.5 Experimental Throughput Performance of PIT RC/QC-MIMO . . . 147

9 Conclusions

9.1 Conclusions . . . . . . . . . . . . . . . . . . . 151

9.2 Future work . . . . . . . . . . . . . . . . 153

$\begin{array}{ll}\text { List of References } & 155\end{array}$ 
Appendix A UCD Properties and Algorithm

A.1 Reduced Complexity UCD Algorithm . . . . . . . . . . . . . 158

A.2 UCD SINR Properties $\ldots \ldots \ldots \ldots \ldots \ldots$

Appendix B RC/QC LDPC Codeword Puncturing Technique 161 


\section{List of Tables}

8.1 Comparison of the instantaneous feedback requirements of different MIMO transceivers. . . . . . . . . . . . . . . . . . 128 


\section{List of Figures}

2.1 MIMO system model . . . . . . . . . . . . . . . . . . . 13

3.1 The unconstrained capacity of UT and IT MIMO systems with different numbers of receive and transmit antennas. . . . . . . . . . . 35

3.2 The unconstrained capacity of QR-based BLAST detectors with different numbers of receive and transmit antennas. . . . . . . . . 37

3.3 The unconstrained capacity of GMD-based BLAST detectors with different numbers of receive and transmit antennas. . . . . . . . . . 40

3.4 The unconstrained capacity of ASGMD-based BLAST detectors with different numbers of receive and transmit antennas. . . . . . . . . 41

3.5 BER performance of $2 \times 2$ UT MIMO systems using 16-QAM. . . . . 44

3.6 BER performance of $3 \times 3$ UT MIMO systems using 16-QAM. . . . . . 44

3.7 BER performance of $4 \times 4$ UT MIMO systems using 16-QAM. . . . . 45

3.8 BER performance of UT MIMO systems with linear detectors using 64-QAM. ...................... 46

3.9 BER performance of $2 \times 2$ IT MIMO systems using 16-QAM. . . . . 47

3.10 BER performance of $3 \times 3$ IT MIMO systems using 16-QAM. . . . . . 48

3.11 BER performance of $4 \times 4$ IT MIMO systems using 16-QAM. . . . . 48

3.12 BER performance of $2 \times 2$ IT MIMO systems using 64-QAM. . . . . . 50

3.13 BER performance of $3 \times 3$ IT MIMO systems using 64-QAM. . . . . . 50

3.14 BER performance of $4 \times 4$ IT MIMO systems using 64-QAM. . . . . . 51 
4.1 Coded MIMO system model. . . . . . . . . . . . . . . . . 55

4.2 The Turbo-BLAST detector $\ldots \ldots \ldots \ldots \ldots \ldots$

4.3 Throughput performance of RC/QC LDPC code using different codeword lengths and BPSK in an AWGN channel. . . . . . . . . . . . 69

4.4 Throughput performance of RC/QC LDPC code using different codeword lengths and 4-QAM in an AWGN channel. . . . . . . . 70

4.5 Throughput performance of RC/QC LDPC code using different codeword lengths and 16-QAM in an AWGN channel. . . . . . . 70

4.6 Throughput performance of RC/QC LDPC code using different codeword lengths and 64-QAM in an AWGN channel. . . . . . . . . . 71

4.7 Theoretical throughput performance of rate-constrained $2 \times 2$ MIMO transceivers. . . . . . . . . . . . . . . . 73

4.8 Theoretical throughput performance of rate-constrained $3 \times 3$ MIMO

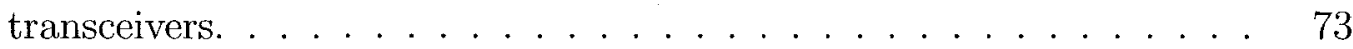

4.9 Theoretical throughput performance of rate-constrained $4 \times 4$ MIMO

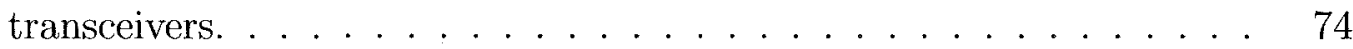

4.10 Theoretical throughput performance worst subchannel of $4 \times 4$ MIMO

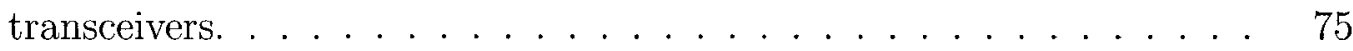

4.11 Experimental throughput performance of $2 \times 2 \mathrm{RC} / \mathrm{QC}-\mathrm{MIMO}$ with dif-

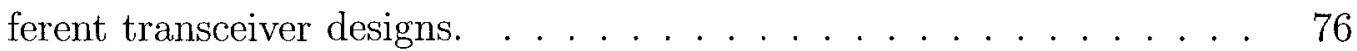

4.12 Experimental throughput performance of $3 \times 3 \mathrm{RC} / \mathrm{QC}-\mathrm{MIMO}$ with dif-

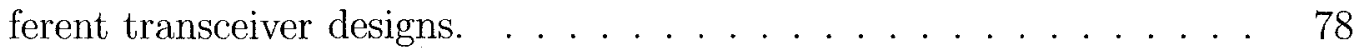

4.13 Experimental throughput performance of $4 \times 4 \mathrm{RC} / \mathrm{QC}-\mathrm{MIMO}$ with different transceiver designs. . . . . . . . . . . . . . 79

5.1 Theoretical constrained capacity of $M$-QAM in an AWGN channel. . 82

5.2 Experimental throughput of 'short' RC/QC LDPC codes with different $M$-QAM constellations in an AWGN channel. . . . . . . . . . . 83 
5.3 Theoretical constrained capacity of $2 \times 2$ SVD-based transceivers using different power and bit-rate allocation algorithms. . . . . . . . . .

5.4 Theoretical constrained capacity of $3 \times 3$ SVD-based transceivers using different power and bit-rate allocation algorithms. . . . . . . . . . 90

5.5 Theoretical constrained capacity of $4 \times 4$ SVD-based transceivers using different power and bit-rate allocation algorithms. . . . . . . . .

5.6 Theoretical constrained capacity of $2 \times 2$ ASGMD-based transceivers using different antenna selection algorithms. . . . . . . . . . . . 91

5.7 Theoretical constrained capacity of $3 \times 3$ ASGMD-based transceivers using different antenna selection algorithms. . . . . . . . . . . . 92

5.8 Theoretical constrained capacity of $4 \times 4$ ASGMD-based transceivers using different antenna selection algorithms. . . . . . . . . .

5.9 Experimental throughput of $2 \times 2 \mathrm{RC} / \mathrm{QC}-\mathrm{MIMO}$ transceivers with different transceiver designs. . . . . . . . . . . . . . . . 94

5.10 Experimental throughput of $3 \times 3 \mathrm{RC} / \mathrm{QC}-\mathrm{MIMO}$ transceivers with different transceiver designs. . . . . . . . . . . . . . . .

5.11 Experimental throughput of $4 \times 4$ RC/QC-MIMO transceivers with different transceiver designs. . . . . . . . . . . . . . .

6.1 BER performance of an uncoded $2 \times 2$ MIMO system using MMSE detectors and 16-QAM. . . . . . . . . . . . . . 104

6.2 Number of exponential computations performed by the $2 \times 2$ MIMO system using T-SS/LLRE and MMSE-ISF detector and 16-QAM for $N_{I}=1$.

6.3 BER performance of an uncoded $3 \times 3$ MIMO system using MMSE detectors and 16-QAM. . . . . . . . . . . 107

6.4 BER performance of an uncoded $4 \times 4$ MIMO system using MMSE detectors and 16-QAM. . . . . . . . . . . . . . 107 
6.5 BER performance of an uncoded $2 \times 2$ MIMO system using MMSE detectors and 64-QAM. . . . . . . . . . . . . 108

6.6 Number of exponential computations performed by the $2 \times 2$ MIMO system using the T-SS/LLRE and MMSE-ISF detector and 64-QAM for $N_{I}=1 \ldots \ldots \ldots \ldots \ldots$

6.7 BER performance of an uncoded $3 \times 3$ MIMO system using MMSE detectors and 64-QAM. . . . . . . . . . . . . . . . 110

6.8 BER performance of an uncoded $4 \times 4$ MIMO system using MMSE detectors and 64-QAM. . . . . . . . . . . . . . . 111

6.9 Simulated throughput performance of $2 \times 2$ IT MIMO systems when coupled with RC/QC LDPC codes. . . . . . . . . . . . . . . . . 112

6.10 Simulated throughput performance of $3 \times 3$ IT MIMO systems when coupled with RC/QC LDPC codes. . . . . . . . . . . . . . 113

6.11 Simulated throughput performance of $4 \times 4$ IT MIMO systems when coupled with RC/QC LDPC codes. . . . . . . . . . . . . . 114

7.1 Simulated throughput performance of $2 \times 2$ RC/QC-ASSVD and -ASGMD for a normalized channel. . . . . . . . . . . . . . 117

7.2 Experimental throughput performance of $2 \times 2$ IT Hybrid transceiver when coupled with RC/QC LDPC codes. . . . . . . . . . . . . . 119

7.3 Experimental throughput performance of $3 \times 3$ IT Hybrid transceiver when coupled with RC/QC LDPC codes. . . . . . . . . . . . . . .

7.4 Experimental throughput performance of $4 \times 4$ IT Hybrid transceiver when coupled with RC/QC LDPC codes. . . . . . . . . . . . . . 120

8.1 BER performance of $2 \times 2$ MIMO systems with different quantization strategies and SIC done using the ideal equivalent channel matrix. . .

8.2 BER performance of a $2 \times 2$ GMD-SIC transceiver with different quantization strategies and SIC done using the equivalent channel matrix. 
8.3 BER performance of a $2 \times 2$ GMD-MMSE with different levels of quantization with hard MMSE-SIC nulling vectors matched to the equivalent channel matrix. ...................... . . 14

8.4 BER performance of a $3 \times 3$ GMD-MMSE with different levels of quantization with hard MMSE-SIC nulling vectors matched to the equivalent channel matrix. . . . . . . . . . . . . . . . . . 142

8.5 BER performance of a $4 \times 4$ GMD-MMSE with different levels of quantization with hard MMSE-SIC nulling vectors matched to the equivalent channel matrix. . . . . . . . . . . . . . . . . 143

8.6 BER performance of a $2 \times 2$ GMD-MMSE with a MMSE-SIC detector matched to the equivalent channel, comparing the importance of magnitude versus phase information. . . . . . . . . . . . . . 145

8.7 BER performance of a $3 \times 3$ GMD-MMSE with a MMSE-SIC detector matched to the equivalent channel, comparing the importance of magnitude versus phase information. . . . . . . . . . . . 14

8.8 BER performance of a $4 \times 4$ GMD-MMSE with a MMSE-SIC detector matched to the equivalent channel, comparing the importance of magnitude versus phase information. . . . . . . . . . . . . 146

8.9 Experimental throughput performance of $2 \times 2$ SVD throughput performance with PQ quantizer. . . . . . . . . . . . . . . . 148

8.10 Performance degradation for $2 \times 2$ transceivers using PQ quantizer. . . 149

8.11 Experimental throughput performance of $2 \times 2$ transceivers using $\mathrm{PQ}$ quantizer. . . . . . . . . . . . . . . . . . 149

8.12 Experimental throughput performance of $3 \times 3$ transceivers using $\mathrm{PQ}$

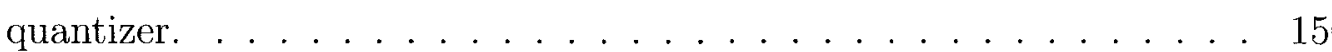




\section{List of Acronyms}

Acronym

3GPP2

AEQ

APP

ARR

ASGMD

BLAST

BER

BP

bps

CCAS

CRC

CSI

CWFA

D-BLAST

ECC

EDC

FDD

FT-SS/LLRE

GMD
Definition

3rd Generation Partnership Project 2

Page

All Entries Quantized

13

A Posteriori Probability

138

Average Realizable Rate

63

66

Antenna Selection Geometric Mean Decomposition

28

Bell Labs Layered Space-Time

Bit Error Rate

2

Belief Propagation

57

Bits Per Second

1

Constrained Capacity Antenna Selection 87

Cyclic Redundancy Check

55

Channel State Information

3

Constrained Water-Filling Algorithm

7

Diagonal Bell Labs Layered Space-Time 3

Error-Control Coding

4

Error-Detection Code

55

Frequency Division Duplex 4

Fully Tuned SS/LLRE

100

Geometric Mean Decomposition 
H-ARQ Hybrid Automatic-Repeat-Request 5

$\begin{array}{lll}\text { HS-SIC Hard Symbol SIC } & 17\end{array}$

$\begin{array}{lll}\text { i.i.d. } & \text { Independent Identically Distributed }\end{array}$

$\begin{array}{lll}\text { IT } & \text { Informed Transmitter }\end{array}$

$\begin{array}{lll}\text { LCU Look-Ahead Carry Unit } & 57\end{array}$

LDPC Low Density Parity Check 5

$\begin{array}{lll}\text { LLR } & \text { Log-Likelihood Ratio }\end{array}$

$\begin{array}{lll}\text { MAI } & \text { Multiple Access Interference } & 7\end{array}$

MCW Multiple Codeword $\quad 65$

MIMO Multiple Input Multiple Output 1

ML Maximum Likelihood 2

MMSE $\quad$ Minimum Mean Squared Error 21

$\begin{array}{lll}\text { NCWFA } & \text { Numerical Constrained Water-Filling Algorithm } & 83\end{array}$

$\begin{array}{lll}\text { pdf } & \text { Probability Density Function } & 97\end{array}$

$\begin{array}{lll}\text { PIT } & \text { Partially Informed Transmitter } & 38\end{array}$

$\begin{array}{lll}\text { PoI } & 18\end{array}$

$\begin{array}{lll}\mathrm{PQ} & \text { Parameterized Quantizer } & 6\end{array}$

$\begin{array}{lll}\text { PQL } & \text { Parameterized Quantizer Long Interval }\end{array}$

QAM Quadrature Amplitude Modulation 12

RC Rate-Compatible 5

RC/QC Rate-Compatible Quasi-Cyclic 5

SCW Single Codeword $\quad 65$

$\begin{array}{lll}\text { SIC Successive Interference Cancellation } & 17\end{array}$

SINR Signal-to-Interference and Noise Ratio 25

SISO $\quad$ Single-Input Single-Output 1

SM Spatial Multiplexing 2

SNR Signal-to-Noise Ratio 1 
SS/LLRE

STBC

SVD

T-SS/LLRE

TDD

UCAS

UCD

UT

V-BLAST

WFA
Soft-Symbol and LLR Estimator

Space-Time Block Code

2

Singular Value Decomposition

24

Tunable SS/LLRE

7

Time Division Duplex 4

Unconstrained Capacity Antenna Selection

91

Uniform Channel Decomposition

3

Uninformed Transmitter

23

Vertical Bell Labs Layered Space-Time

3

Water-Filling Algorithm 


\title{
List of Mathematical Operators and
}

\section{Symbols}

\author{
Operators \\ Description \\ $1 \cdot 1$ \\ Magnitude operator. \\ $\|\cdot\|_{F}^{2}$ \\ Frobenius norm. \\ $\langle\cdot, \cdot\rangle$ \\ Dot product of two vectors. \\ $\angle$ \\ Angle of complex number. \\ $\mathrm{E}[\cdot]$ \\ Expectation operator. \\ $f_{A}(A \mid B)$ \\ Likelihood function for an event $A$ given $B$ occurred. \\ $\mathrm{G}_{a}$ \\ Augmented matrix of a matrix $\mathbf{G}$. \\ $\operatorname{Pr}\{A\}$ \\ Probability of an event A. \\ $\operatorname{Tr}\{\cdot\}$ \\ Trace of a matrix.
}

$\underline{\text { Symbol }}$

$\eta_{\min }$

$\gamma$

$\gamma_{d B}$

$\gamma_{\max }$

$\lambda$

$\Lambda$
Description

Minimum nominal rate of a RC/QC-MIMO system.

SNR.

SNR in $\mathrm{dB}$.

SNR for which rate of RC/QC LDPC code is six. $\quad 86$

Log-likelihood ratio.

Diagonal matrix of SVD.
85

62

$\underline{\text { Page }}$

80

1 86 


\begin{tabular}{|c|c|c|}
\hline$\nu$ & Spatially filtered noise vector. & 14 \\
\hline$\Omega$ & The number of feedback bits for the magnitudes of $\mathrm{PQ}$. & 137 \\
\hline$\Omega_{1}$ & Semi-unitary precoder matrix of SVD, GMD and UCD. & 4 \\
\hline$\Omega_{2}$ & Second semi-unitary precoder matrix of UCD. & 9 \\
\hline$\Phi$ & Power allocation matrix. & 4 \\
\hline$\phi_{i}$ & Power allocated to the $i^{t h}$ subchannel. & 55 \\
\hline$\Psi$ & Phase matrix of PQ method. & 134 \\
\hline$\rho_{i}$ & SINR of the $i^{t h}$ subchannel. & 5 \\
\hline$\sigma_{i}$ & The $i^{t h}$ singular value of a matrix. & 5 \\
\hline $\bar{\sigma}$ & The geometric mean of the singular values of a matrix. & 27 \\
\hline$\sigma_{\star}^{2}$ & The variance of a random variable $\star$. & 5 \\
\hline$\Theta$ & The number of feedback bits for the phases of PQ. & 137 \\
\hline$\Upsilon$ & Set of Givens rotation matrix of PQ method. & 134 \\
\hline $\mathcal{B}$ & The set of constellation sizes used in adaptive modulation. & 1 \\
\hline $\mathcal{C}$ & The set of considered interferers for T-SS/LLRE. & 101 \\
\hline $\mathbf{c}$ & The LDPC codeword. & 13 \\
\hline $\mathrm{C}$. & The instantaneous unconstrained capacity of a system. & 32 \\
\hline $\bar{C}$ & The ergodic unconstrained capacity of a system. & 34 \\
\hline$C_{C N}$ & The instantaneous constrained capacity of a system. & 72 \\
\hline $\mathrm{D}$ & The MMSE nulling matrix. & 22 \\
\hline \multirow[t]{2}{*}{$\mathrm{d}_{i}$} & The $i^{t h}$ MMSE nulling vector. & \\
\hline & Average energy of a QAM symbol. & \\
\hline $\mathbf{F}$ & The precoder matrix of a MIMO system. & \\
\hline G & The equivalent channel matrix. & \\
\hline $\mathbf{G}_{c}$ & The LDPC code generator matrix. & \\
\hline $\mathbf{G}_{I T}$ & The equivalent channel matrix of an IT. & \\
\hline $\mathbf{G}_{P I T}$ & The equivalent channel matrix of a PIT. & \\
\hline
\end{tabular}


The rank of channel matrix $\mathbf{H}$.

The bit length of message word $\mathbf{m}^{\prime}$.

The number of subchannels used.

Incremental redundancy block size.

Number of iterations used by the BP decoder.

The QAM constellation size used for subchannel $i$.

The total number of bits transmitted for one channel use. 13

$\mathbf{m}^{\prime}$

The message word.

The CRC padded message word.

The noise power spectral density.

The bit length of codeword, $\mathbf{c}$.

The total number of considered MAI realizations.

Number of Givens rotations for PQ method.

Number of receive antennas in a MIMO system.

Number of successfully detected codewords. 


$\begin{array}{lll}\mathbf{Q}_{s} & \text { The symbol correlation matrix. } & 33 \\ \mathbf{Q} & \text { The left-hand unitary matrix of GMD. } & 26 \\ \mathbf{Q}_{z} & \text { The unitary matrix of the QR-decomposition. } & 20 \\ \mathbf{r} & \text { The received samples for the MIMO system. } & 14 \\ \mathbf{R} & \text { The upper triangular matrix of GMD. } & 26 \\ \mathbf{R}_{c} & \text { The code rate of a LDPC code. } & 56 \\ \mathbf{R}_{c}^{(m i n)} & \text { The minimum code rate of a RC/QC LDPC code. } & 67 \\ \mathbf{R}_{z} & \text { The upper triangular matrix of the QR-decomposition. } & 20 \\ \mathbf{S} & \text { The modulated symbol vector. } & 13 \\ s_{i} & \text { The } i^{t h} \text { modulated symbol. } & 13 \\ \bar{s}_{i} & \text { The expectation of symbol } s_{i} . & 59 \\ \hat{s}_{i} & \text { The estimate of symbol } s_{i} . & 17 \\ \mathbf{s}_{i, l} & \text { A MAI realization for symbol } s_{i} . & 99 \\ \mathcal{S}_{i} & \text { The constellation that } s_{i} \text { is selected from. } & 59 \\ \mathbf{U} & \text { The left-hand unitary matrix of SVD. } & 25 \\ \mathbf{V} & \text { The right-hand unitary matrix of SVD. } & 25 \\ \mathbf{W} & \text { The spatial filtering matrix. } & 14 \\ \mathbf{w}_{i} & \text { A row vector of W. } & 15 \\ \mathbf{x} & \text { The precoded symbol vector. } & 15 \\ \mathbf{y} & \text { The spatially filtered received sample vector. } & 14 \\ & \text { The decision variable vector. } & \\ & & \end{array}$




\section{Chapter 1}

\section{Introduction}

\subsection{Background and Motivation}

\subsubsection{MIMO: The Metamorphosis}

For over a decade, wireless communication systems that employ multiple-input and multiple-output (MIMO) antennas at the transmitter and receiver have been the focus of intense research. This is mostly due to the promised increase in spectral efficiency that MIMO systems have over their single-input single-output (SISO) counterparts. For example, a SISO additive white Gaussian noise (AWGN) channel is well-known to have a capacity of

$$
C_{A W G N}(\gamma)=\log _{2}(1+\gamma) \mathrm{bps} / \mathrm{Hz}
$$

where $\gamma$ is the signal-to-noise ratio (SNR); and bps stands for bits per second. By comparison, the instantaneous spectral efficiency of a $N \times N$ MIMO system, can be as large as [1]

$$
C_{M I M O}(\gamma)=N \log _{2}\left(1+\frac{\gamma}{N}\right) \text { bps } / \mathrm{Hz}
$$

Although the term inside the logarithm of (1.2) grows slower than the term inside (1.1) as $N$ increases, the factor of $N$ outside of the logarithm has a larger impact on the spectral efficiency of the wireless system because this term grows linearly, 
which is faster than logarithmic growth. For example, a $2 \times 2$ MIMO system can have a spectral efficiency of $6.92 \mathrm{bps} / \mathrm{Hz}$ at a SNR of $20 \mathrm{~dB}$. By contrast the SISO AWGN transceiver only achieves a spectral efficiency of $4.39 \mathrm{bps} / \mathrm{Hz}$. The addition of extra transmit and receive antennas comes at no extra bandwidth cost, it just requires appropriate spacing between antennas such that they have uncorrelated fading channels. This makes MIMO an even more attractive enhancement for the wireless system. Of course, there are space limitations for applications such as cellular devices operating near the $1 \mathrm{GHz}$ range. However, there are devices such as laptops, wireless local area networks (WLANs), satellites, wireless base stations, etc. for which MIMO systems can be feasibly deployed.

Armed with this theoretical capacity formula, engineers have been emboldened to explore this "new frontier" in wireless communications. Recognizing that typical solutions for the SISO wireless system did not directly translate into the MIMO system, new solutions were suggested. A major problem identified in implementing MIMO systems is in the signal detection process. The optimal detector for a SISO system is the maximum likelihood (ML) detector. The same is true for the MIMO system; however, the number of computations involved for MIMO ML grows exponentially with the number of and constellation size of the symbols transmitted. This is just one problem that needed to be addressed; and it was, from two perspectives: the first came with the introduction of the space-time block code (STBC) of Alamouti [2] and was followed by numerous other papers on the subject (for example [3-5]); the second allows for spatial multiplexing (SM), where all transmit antennas send a different signal and these signals are detected separately using linear filters $[6,7]$. The major drawback of using STBCs is that they reduce the achievable throughput of a MIMO system in order to achieve better bit error rate (BER) by obtaining higher signal diversity through multiple copies of the same 
signal. MIMO systems operating on the principle of SM are able to attain the promised spectral efficiency of (1.2). This was shown using the D-BLAST (Diagonal Bell Labs Layered Space-Time) algorithm [6]; however, the system is very complex to implement and a reduced-complexity version called V-BLAST (Vertical BLAST) was suggested. However, implemented on its own, the V-BLAST algorithm suffers from low diversity and, as a result, poor BER performance. An effort had to be made to reconcile the diversity gains of STBCs with the spectral efficiency of V-BLAST.

\subsubsection{Joint Transceiver Design: The Channel Decomposition Method}

In [8], it was shown that the diversity and multiplexing gain can be achieved simultaneously. This finding forced researchers to re-think their design criteria and search for a model that jointly achieves a good tradeoff between diversity and SM. One direction taken was the inclusion of channel state information (CSI) at the transmitter. This CSI could then be jointly used by the transmitter and receiver to design a joint transceiver that would have a good diversity and SM tradeoff. A capacity achieving method is proposed by Raleigh et. al. [9], but this solution is not designed with the assumption that the MIMO transceiver has constrained bit-rate. In his Ph.D. dissertation [10], Jiang explores channel-decomposition-based techniques for jointly designing transceivers that meet the diversity/SM tradeoff. One of his proposals, uniform channel decomposition (UCD), can achieve full MIMO diversity and is capacity achieving. However, this system is designed under the pretence that full CSI is available to the MIMO transmitter. Depending upon the implementation of a wireless system this is not always true without some sort of feedback mechanism from the receiver. Given a slow-fading channel, a time 
division duplex (TDD) wireless system the channel seen by both the transmitter and receiver are assumed to be the same, and in this case no feedback would be required. However, for a frequency division duplex (FDD) system this is not the case. For a non-TDD wireless system to be able to send CSI feedback information back to the transmitter, the channel must not change much over the time for which this information is relayed back. Practically, it is better that the channel not change over many multiple transmissions so that there is low feedback overhead.

For wireless systems with a frequency flat slow-fading channel model, using jointly designed transceivers, the amount of feedback sent back to the transmitter in order to fully construct the transmitter's precoder is infinite. However, in real-world systems the amount of feedback must be limited as the channel will surely have changed over an infinite amount of time, not to mention the delay that would be incurred in waiting to transmit the signal. It is obvious that the amount of CSI feedback must be capped. There are no limited-CSI designs for the transceivers described in [9] and [10]. Developing a methodology for building their precoders using a limited amount of feedback is an open problem.

\subsubsection{Achieving Throughput Close to Capacity: An Adapt- able Error-Control Code}

Joint transceivers are rarely designed with error-control coding (ECC) in mind. Most transceivers are designed to optimize some set of parameters such as the SNR, BER or capacity of the MIMO system. In the case where the transceiver design attempts to optimize the capacity of a MIMO system, an unconstrained bit-rate and the availability of some perfect ECC, capable of achieving capacity for all instantaneous SNRs is assumed. Some MIMO systems have been combined with ECC codes (for 
example [11-14]), but in most cases these only achieve one rate or a very small set of different rates. Of these papers, [13] and [14] can achieve multiple coding rates.

In [13] MIMO STBCs are combined with LDPC codes. Using a variety of modulation schemes and by puncturing a single LDPC "mother" code, a method for achieving a small set of nominal rates is proposed. However, this system does not specify how to select the appropriate rate, nor does it use any incremental redundancy, so that if there is a decoder failure, the entire codeword must be retransmitted. In addition, the use of a STBC does not allow the MIMO system to achieve the highest possible spectral efficiency.

In [14] MIMO is combined with a set of LDPC codes and adaptive modulation. A Type-I hybrid automatic-repeat-request (H-ARQ) scheme for incremental redundancy. The appropriate LDPC code and modulation on each subchannel are specified by a selection algorithm that operates on the receiver SNR of the MIMO system. This system requires extra memory to store every LDPC code and can only achieve a small set of nominal rates. The use of a Type-I H-ARQ leads to extra overhead for decoder failures as the entire codeword must be re-transmitted. In addition, the transmitter is not aware of the channel statistics and, as a result, is not able to take full advantage of the MIMO system.

The rate-compatible Quasi-Cyclic (RC/QC) low-density parity-check (LDPC) codes of [15], combined with adaptive modulation and implemented using Type-II H-ARQ provide a flexible, low-overhead method to obtain a large set of discrete rates. In addition, MIMO with CSI available to the transmitter is a good way to improve the spectral efficiency of a communication system. The combination of RC/QC LDPC codes with MIMO (RC/QC-MIMO) and adaptive modulation is studied in 
this thesis.

\subsubsection{A Combined Approach: A Low-Complexity MIMO Ar- chitecture for Achieving High Throughput}

Some major implementation issues indentified as obstacles to the MIMO system's ability to attain high throughput are: the diversity/SM tradeoff of a MIMO system with constrained rate; limited feedback strategies for building a MIMO precoder of a jointly designed transceiver; and the need for a methodology for achieving a wide variety of rates. In this thesis we address these design issues and attempt to design a communication system with low computational complexity and overhead that is able to achieve a high overall throughput.

The transceivers that we implement are based on those proposed in $[9,10]$. We make some small modifications to these transceivers, where needed, in order to implement them in a MIMO system with constrained capacity. In addition, we propose a simple low-complexity parameterized quantization (PQ) method for quantizing the precoder information necessary for implementing these transceivers. Using $\mathrm{RC} / \mathrm{QC}$ LDPC codes of [15] with a MIMO system that employs adaptive modulation allows for a communication system that is adaptable to the channel fluctuations of the wireless medium. The RC LDPC code operation is adapted to be integrated into the MIMO system using a Type-II H-ARQ protocol. This coded MIMO system is evaluated for a variety of transceivers and from this evaluation a new Hybrid transceiver is proposed. The combination of the Hybrid transceiver with $\mathrm{RC} / \mathrm{QC}$ LDPC codes and a $\mathrm{PQ}$ quantizer produces a real-world ready MIMO transceiver capable of achieving high spectral efficiency. 


\subsection{List of Contributions}

The following is a list of what are considered to be the most significant contributions to scientific knowledge made by this thesis.

\section{1) CWFA: A new rate constrained power and bit-rate allocator}

The constrained water-filling algorithm (CWFA) is a recursive version of the wellknown water-filling algorithm (WFA). It is designed for use in rate-constrained MIMO systems. The results in this thesis show that the CWFA has better constrained throughput performance than WFA and as a result is better suited than WFA for the RC/QC-MIMO system.

\section{2) T-SS/LLRE for layered BLAST detection}

A Tunable soft-symbol and log-likelihood ratio estimator (T-SS/LLRE) is designed for layered BLAST detection, where there is multiple access interference (MAI) in the decision variables. Typically, most systems model MAI as a Gaussian random variable, but this is not actually the case. The T-SS/LLRE is able to adaptively model the MAI statistics. Using T-SS/LLRE with iterative soft MMSE filtering in a RC/QC-MIMO system is shown to give better performance than the Gaussian SS/LLRE. In addition, T-SS/LLRE is shown to add diversity to a layered BLAST detector, which usually has none.

\section{3) Hybrid transceiver}

Given the experimental throughput results given in this thesis a Hybrid transceiver is proposed. This Hybrid transceiver selects between two transceivers and then does antenna selection. The RC/QC-Hybrid system is shown to have the best overall throughput performance of all transceivers in this thesis that do not require the 
feedback of a power allocation matrix.

\section{4) Parameterized quantizer for semi-unitary matrices}

A parameterized quantization method is developed to characterize a semi-unitary precoder matrix. This quantization method is based upon a set of Givens rotations that are represented by a phase and magnitude parameter. Use of this method was shown to greatly reduce the amount of feedback needed to achieve BER performance near that of a MIMO system with perfect CSI knowledge.

\subsection{List of Publications Resulting from this Work}

\section{CCECE 2008 Paper}

- A paper, [16], was accepted and published in the conference proceedings of the 2008 Canadian Conference on Electrical and Computer Engineering. The paper proposed the parameterized quantization strategy discussed in this thesis and also gave some suggestions on the type of detection that ought to be employed given quantization errors occur in the precoder matrix.

\subsection{Thesis Outline}

The opening portion of this thesis, Part I, is composed of two chapters and is dedicated to giving a comprehensive study of channel- decomposition-based MIMO transceivers. In Chapter 2, a general MIMO system model is described. For this system model many MIMO transceivers with uninformed and informed transmitter are described. In Chapter 3, the transceivers described in Chapter 2 are studied in terms of their unconstrained capacity and BER performance. 
Part II describes the implementation of a rate-constrained coded MIMO system where the transmitter has full knowledge of the CSI. Part II is composed of four chapters. In Chapter 4, an implementation of the RC/QC-MIMO system model is described in detail. In addition, the transceivers described in Chapter 2 are evaluated in terms of their theoretical constrained capacities. Chapter 4 also describes the throughput metric that is used to quantify experimental results for the different RC/QC-MIMO systems. In Chapter 5, a new rate-constrained power and bit-rate allocation algorithm is proposed. Further, an antenna selection algorithm for the rate-constrained MIMO system is introduced. These new algorithms are then evaluated with the different RC/QC-MIMO transceivers in terms of their theoretical constrained capacity and experimental throughput performance. In Chapter 6, a new soft-symbol and log-likelihood ratio estimator (SS/LLRE) is designed for layered BLAST detectors that use soft nulling vectors. The BER and experimental throughput performance of the new SS/LLRE is also given in this chapter. Using the experimental results given in Chapters $4-6$, a new Hybrid transceiver is proposed for the RC/QC-MIMO system in Chapter 7. The performance of this new RC/QC-Hybrid system is evaluated in terms of its experimental throughput performance.

Part III contains one chapter, which is dedicated to studying the effects that limited CSI feedback has on RC/QC-MIMO. Chapter 8 focuses on MIMO transceivers that do not require the feedback of a power allocation matrix. A parameterized quantization strategy for quantizing a semi-unitary matrix is designed and evaluated using BER, to find out how many bits of feedback are required to attain near-perfect CSI performance. In addition, Chapter 8 gives the experimental throughput performance when a limited amount of feedback is used to quantize the semi-unitary precoder matrix of a MIMO transceiver. 
Chapter 9 gives a summary of the work performed and results obtained in this thesis, as well as suggests some topics for future work that this thesis inspires. 


\section{Part I}

A Comprehensive Study of

Channel Decomposition-Based

MIMO Transceivers 


\section{Chapter 2}

\section{MIMO Systems}

In this chapter we introduced a system model for MIMO. Layered detection is elaborated upon as a low-computational-complexity method to separate and detect symbols for a SM MIMO system. Several transceiver designs are described as components of the system model.

\subsection{MIMO System Model}

In this thesis we consider a MIMO system with $N_{r}$ receive and $N_{t}$ transmit antennas, where the channel is modelled by frequency flat Rayleigh fading. Figure 2.1 gives the general structure of our MIMO system.

\subsubsection{Symbol Mapping}

The transmitter combines $L$ different quadrature amplitude modulation (QAM) symbols for simultaneous transmission during each MIMO channel use. The modulation order (bits per each QAM symbol) is adaptive, based on CSI as measured by the receiver and delivered to the transmitter via a feedback channel. For $i \in\{1,2, \ldots, L\}$, let $M_{i}$ denote the constellation size of the $i^{\text {th }}$ QAM symbol. 


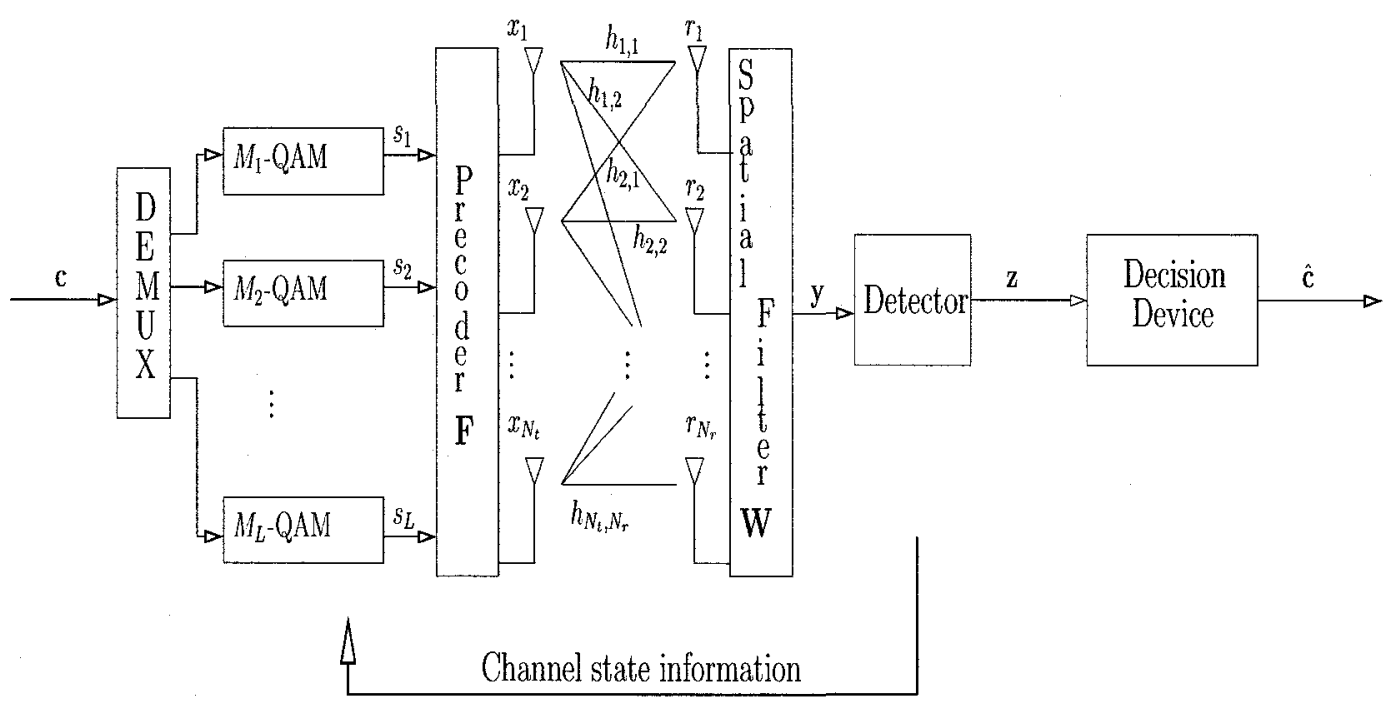

Figure 2.1: MIMO system model

A message vector, $\mathbf{c}$, with a length of $m_{T}=\sum_{i=1}^{L} \log _{2}\left(M_{i}\right)$ bits is partitioned into $L$ sub-blocks, and each sub-block is mapped to a QAM symbol. In particular, the $i^{\text {th }}$ sub-block (which consists of $\log _{2}\left(M_{i}\right)$ bits) is mapped to a point $s_{i}$, in a Gray mapped $M_{i}$-QAM symbol constellation. Although each constellation has the same fixed average energy, $E_{s}$, adaptive power control for each symbol can be supported as part of the precoder. The mappings for the QAM constellations used in this thesis are defined in the 3GPP2 (3rd Generation Partnership Project 2) standard [15].

\subsubsection{Precoder}

The QAM symbol vector, $\mathbf{s}=\left[s_{1}, s_{2}, \ldots, s_{L}\right]^{T}$ (where the superscript $T$ denotes the matrix transpose), filtered by a $N_{t} \times L$ precoder matrix, $\mathbf{F}$, to generate the transmitted vector $\mathbf{x}=\left[x_{1}, x_{2}, \ldots, x_{L}\right]^{T}$ according to

$$
\mathrm{x}=\mathbf{F} \text {. }
$$


The precoder matrix $\mathbf{F}$ is subject to a power constraint so that the total energy per channel use is

$$
\begin{aligned}
\operatorname{Tr}\left\{\mathrm{E}\left[\mathbf{x x}^{+}\right]\right\} & =\sum_{j=1}^{N_{t}} \mathrm{E}\left[\left|x_{j}\right|^{2}\right] \\
& =N_{t} E_{s}
\end{aligned}
$$

where $\operatorname{Tr}\{\cdot\}$ denotes the trace of a matrix, $\mathrm{E}[\cdot]$ denotes expected value, and the superscript + denotes the Hermitian transpose.

\subsubsection{Discrete-time MIMO channel}

The received samples from one MIMO channel use is

$$
\mathbf{r}=\mathbf{H x}+\mathbf{n}
$$

where $\mathbf{r}$ is the $N_{r} \times 1$ received sample vector; $\mathbf{H}$ is the $N_{r} \times N_{t}$ channel matrix with all entries $\left\{h_{i, j}\right\}$ being independent identically distributed (i.i.d.) circularly complex zero-mean Gaussian random variables with unit variance; and $\mathbf{n}$ is an $N_{r} \times 1$ AWGN noise vector with all entries $n_{i}$ being i.i.d. circularly complex zero-mean Gaussian random variables with variance $N_{0}$.

\subsubsection{Spatial Filter}

The received sample vector is spatially filtered to produce

$$
\begin{aligned}
\mathbf{y} & =\mathrm{Wr} \\
& =\mathrm{WHFs}+\nu \\
& =\mathrm{Gs}+\nu
\end{aligned}
$$

where $\mathbf{W}$ is the $N_{r} \times N_{r}$ spatial filter; $\mathbf{G}$ is the $N_{r} \times N_{t}$ equivalent channel matrix; and $\nu$ is the $N_{r} \times 1$ filtered noise vector with each entry $\nu_{i}$ being a complex zero-mean 
Gaussian random variable with variance $\sigma_{\nu_{i}}^{2}=\left|\mathbf{w}_{i}\right|^{2} N_{0}$; where $\mathbf{w}_{i}$ is the $i^{\text {th }}$ row vector of W.

\subsubsection{Detector}

The detector takes as input the spatially filtered received vector $\mathbf{y}$ and generates decision variables for the transmitted QAM symbols, $\mathbf{z}$. Let $\mathbf{z}=\left[z_{1}, z_{2}, \ldots, z_{L}\right]^{T}$ denote these estimates. Some MIMO symbol detection methods are described in the next section.

\subsubsection{Decision Device}

The decision device uses the decision variable vector, $\mathbf{z}$, to find the closest mapping in Euclidean space that corresponds to the estimate of the bits in the transmitted message $\mathbf{c}$.

In this chapter we introduce some suggested designs for MIMO systems that manipulate parameters $\mathbf{F}$ and $\mathbf{W}$ to obtain better BER and capacity performance.

\subsection{MIMO with an Uninformed Transmitter}

In this section we investigate MIMO transceivers where the transmitter is not aware of the channel conditions. In such an environment the transmitter cannot be adaptive, so there is no precoding, power or bit loading (i.e. $\mathbf{F}=\mathbf{I}_{N_{t}}, L=N_{t}$ and $M_{i}$ is the same for all $i$ ). This also means that the transceiver complexity is mainly at the receiver. 


\subsubsection{Maximum Likelihood Detector}

The ML detector is the optimal detector for minimizing the probability of symbol detection error. It jointly detects the entire symbol block of a single channel. For the ML detector, the decision variable vector $\mathbf{z}=\mathbf{y}$. The decision device of Figure 2.1 uses the decision variable vector to find a symbol vector that minimizes

$$
\hat{\mathbf{s}}=\arg \min _{\hat{\mathbf{s}} \in \mathbf{s}}\|\mathbf{z}-\mathbf{G} \hat{\mathbf{s}}\|_{F}^{2}
$$

where $\hat{\mathbf{s}}=\left[\hat{s}_{1}, \hat{s}_{2}, \ldots, \hat{s}_{L}\right]^{T}$ is an estimate of the transmitted symbols and $\|\cdot\|_{F}^{2}$ is the Frobenius norm. The decision device then produces $\hat{\mathbf{c}}$ from the bit message that would create $\hat{\mathbf{s}}$ in the symbol mapping process. The computational complexity of calculating the most likely block of symbols is $\prod_{i=1}^{L} M_{i}$, where $M_{i}$ is the number of points in the constellation used for $s_{i}$. The computational complexity of this algorithm is intractable and difficult to implement practically, should we wish to at rates that exceed single digits. As a result we consider using sub-optimal linear detector techniques.

\subsubsection{The BLAST Detector}

The BLAST detector was first introduced by Foschini et. al. in [7]. It involves sequential symbol-by-symbol detection, combining linear techniques to remove MAI and improve BER performance. These techniques are:

1. Interference cancellation: remove the interference caused by already detected symbols from the received sample,

2. Interference nulling: attempt to suppress interference from yet to be detected symbols, and

3. Ordering: detect symbols that have larger post-filtering SNRs first. 
We describe these techniques in the order that they are given above as it makes for a convenient description. The detector actually uses these techniques in a different order than that which appears above. The order is given at the end of this subsection. This sequential technique of detecting each symbol is often called layered detection. This is because the detection problem can be imagined as a set of layers. At each layer a symbol is concealed by interference and noise. An estimate is made of the symbol at each layer and it is used in detecting the symbols at lower layers.

Interference Cancelling Supposing that the detector has already made a decision on the first $i-1$ symbols of the block, the detector cancels those $i-1$ symbols from the spatially filtered received samples $\mathbf{y}$ to create a pre-filtering variable $\mathbf{u}_{i}$, with

$$
\mathbf{u}_{i}=\mathbf{y}-\sum_{j=1}^{i-1} \mathbf{g}_{j} \hat{s}_{j}
$$

where $\hat{s}_{j}$ is the estimate of the $j^{\text {th }}$ symbol of the block and $\mathbf{g}_{j}$ is the $j^{\text {th }}$ column of $\mathbf{G}$. By substituting (2.3) for $\mathbf{y}$ in (2.5) we see that $\mathbf{u}_{i}$ can be expressed as

$$
\mathbf{u}_{i}=\underbrace{\mathbf{g}_{i} s_{i}}_{\text {desired symbol }}+\underbrace{\sum_{j=1}^{i-1} \mathbf{g}_{j}\left(s_{j}-\hat{s}_{j}\right)}_{\text {canceled interference }}+\underbrace{\sum_{j=i+1}^{L} \mathbf{g}_{j} s_{j}}_{\text {remaining interference }}+\boldsymbol{\nu}
$$

The process of subtracting out previously detected interference has led this detection method to come to be referred to as a successive interference cancellation (SIC) detector [17]. The estimate of $s_{j}$ can be produced using either hard- or soft- symbol decisions. In [7] a hard-symbol interference canceller is used, however in [18] it is shown that the use of a soft-symbol interference canceller can lead to BER performance gain. For simplicity we will focus on describing the hard-symbol SIC (HS-SIC). Soft-symbol will be discussed in Part II.

Hard Symbol Interference Nulling With the assumption of a HS-SIC detector we also assume that all cancelled symbols have been correctly detected. If an 
error is made in detecting a symbol at a higher layer then there will most likely be catastrophic error propagation into the lower layers, making it improbable that correct symbol detection will take place. With soft-symbol SIC detection error propagation is not as likely and the certainty of each estimate can be determined. This leads to different nulling techniques for each system which is why it will be discussed in subsequent chapters.

In the case of HS-SIC, we need only consider the remaining interference in the nulling step. We begin by characterizing the plane of interference (PoI) by the last $i+1$ column vectors of the matrix $\mathbf{G}$. That is, the Gram-Schmidt procedure is used to form an orthonormal basis, $\left\{\mathbf{e}_{i+1}, \mathbf{e}_{i+2}, \ldots, \mathbf{e}_{L}\right\}$, which characterizes the PoI, from the vectors $\left\{\mathbf{g}_{i+1}, \mathbf{g}_{i+2}, \ldots, \mathbf{g}_{L}\right\}$. Then, a vector $\boldsymbol{\eta}_{i}$, orthogonal to the PoI in the direction of $\mathrm{g}_{i}$ is generate by

$$
\boldsymbol{\eta}_{i}=\mathbf{g}_{i}-\sum_{j=i+1}^{L}\left\langle\mathbf{g}_{i}, \mathbf{e}_{j}\right\rangle \mathbf{e}_{j}
$$

where $\langle\cdot, \cdot\rangle$ is the inner product. The filtered received sample vector, $\mathbf{u}_{i}$, is projected onto a normalized version of $\boldsymbol{\eta}_{i}$ to yield the decision variable

$$
z_{i}=\frac{\left\langle\mathbf{u}_{i}, \boldsymbol{\eta}_{i}\right\rangle}{\sqrt{\left\langle\boldsymbol{\eta}_{i}, \boldsymbol{\eta}_{i}\right\rangle}} .
$$

The decision variables are then fed into a symbol-by-symbol ML detectors, within the decision device, which determines the most likely symbol at each layer

$$
\hat{s}_{i}=\arg \min _{s_{i} \in \mathcal{S}_{i}}\left\|z_{i}-s_{i}\right\|^{2}
$$

where $\mathcal{S}_{i}$ is the $M_{i}$-QAM constellation used by the transmitter. Again, the decision device uses $\hat{\mathbf{s}}$ to determine $\hat{\mathbf{c}}$. Separating the traditional blockwise ML detector into a bank of $L$ symbolwise ML detectors reduces the number of computations required to perform symbol detection. The complexity of detection is reduced from exponential to linear with respect to the number of symbols (i.e., from $\prod_{j=1}^{L} M_{j}$ to $\sum_{j=1}^{L} M_{j}$ ), which 
makes it feasible to transmit and detect at higher spectral efficiencies.

It should be noted that when there is no error propagation from incorrect symbol estimation at higher layers of HS-SIC detection, the final symbol to be detected will have full receive diversity. This is because there is no PoI for this symbol. It should also be noted that all symbol energy is conserved since there is no need for projecting the vector. This may not be the case if the actual PoI is considered without assuming perfect interference cancellation. Of course, the danger lies in the naive assumption that no errors will be made at the higher layers of detection. In fact, because all subsequently detected symbols depend upon successful cancellation of interference from previously detected symbols, their BER performance will depend on the accuracy of each previously detected symbol. As a result, the BER performance of all symbols is dependent upon the accuracy of the first symbol to be detected.

Hard Symbol Ordering To achieve the best possible performance the authors of [7] suggest using an ordering algorithm at each layer of detection. They suggest a myopic optimization algorithm. The myopic algorithm detects the symbol that provides the maximum post-projection SNR at the current detection layer. 'That is, given a set $\mathcal{A}$ of remaining indices, symbol

$$
i=\arg \max _{j \in \mathcal{A}}\left|\boldsymbol{\eta}_{j}\right|^{2}
$$

should be detected next.

Choosing the index that maximizes the post-projection SNR at layer $i$ can be done after the interference cancellation step. This way, for each channel realization, $\mathbf{G}$, we will get a different detection ordering. So the order in which detection proceeds is: i) choose the index, $i$, using the myopic ordering algorithm; ii) cancel all previously 
detected symbols; iii) null the remaining interference and decode the symbol using the symbol estimate $\hat{s}_{i} ;$ iv) remove index $i$ from set $\mathcal{A}$.

\subsubsection{The QR Implementation of the BLAST Detector}

\section{Zero Forcing QR BLAST Detection: QR-SIC}

A simple implementation of the BLAST detection algorithm is one that uses the QRdecomposition. The QR decomposition involves decomposing the channel matrix into a $N_{r} \times N_{r}$ unitary matrix, $\mathbf{Q}$, and a $N_{r} \times N_{t}$ upper triangular matrix, $\mathbf{R}$, such that

$$
\mathbf{H}=\mathbf{Q}_{z} \mathbf{R}_{z}
$$

Setting the spatial filter $\mathbf{W}$ to be $\mathbf{W}=\mathbf{Q}_{z}^{+}$and omitting any precoding then $\mathbf{G}=$ $\mathbf{W H F}=\mathbf{R}_{z}$. Recalling that $N_{t}=L$ in the absence of precoding, we can express the resulting detector inputs as

$$
y_{i}=r_{i, i}^{(z)} s_{i}+\sum_{j=1, j \neq i}^{L} r_{i, j}^{z} s_{j}+\nu_{i}
$$

where $\left\{r_{i, j}^{(z)}\right\}$ are the elements of $\mathbf{R}_{z}$. Since $\mathbf{R}_{z}$ is upper triangular, (2.10) reduces to

$$
y_{i}=r_{i, i}^{(z)} s_{i}+\sum_{j=i+1}^{L} r_{i, j}^{(z)} s_{j}+\nu_{i} .
$$

The noise samples, $\nu_{i}$, retain the statistics of $n_{i}$ due to the unitary property of $\mathbf{W}$. We note that the $L^{\text {th }}$ row vector of $\mathbf{R}_{z}$ has only one non-zero entry: $r_{L, L}^{(z)}$. This means that the $L^{\text {th }}$ symbol experiences no MAI from other symbols. Obviously we should begin by detecting this symbol first, and we make this the first layer. We can then cancel its effects from the received samples from other layers. Following this technique, the detector creates decision variables according to

$$
z_{i}=y_{i}-\sum_{j=i+1}^{L} r_{i, j}^{(z)} \hat{s}_{j}
$$


where the detector has the option of being categorized as a HS-SIC or soft-symbol SIC detector, depending on how $\hat{s}_{j}$ is determined. Again, in this chapter we focus on HS-SIC. The QR-BLAST detector is based on the same principles as the BLAST detector. They both cancel the interference caused by previously detected symbols. They both use vector nulling based upon the Gram-Schmidt method ${ }^{1}$. In fact, the QR-SIC detector is an unordered BLAST detector. If we wished to do ordered QR-SIC detection we would have to recalculate the QR decomposition on the undetected layers after each symbol is detected. The ordering would follow that governed by the same myopic algorithm used in ordered BLAST detection.

The main advantage of the QR-SIC detector is its simplicity. Vector nulling is only performed once and decision variable calculations are easily performed using (2.12). The QR decomposition can be calculated using a set of $2 \times 2$ Givens rotations, which has a computation complexity of $\mathrm{O}\left(N_{r}^{2}\right)$. This has relatively low computational complexity even for a large array of antennas. The major drawback of using the QR decomposition is that it yields poor performance at low SNRs and does not achieve receive diversity [18].

\section{Minimum Mean Squared Error QR BLAST Detection: MMSE-SIC}

The minimum mean squared error (MMSE) detector is classically computed using a set of matrix multiplications and inversions. Matrix multiplications and inversion have a computational complexity of $\mathbb{O}\left(N_{r}^{3}\right)$; a number that gets fairly large for larger numbers of receive antennas. In 2000, Hassibi devised a reduced complexity algorithm for calculating the MMSE based upon the BLAST detection algorithm [19]. He devised the scheme by making use of the QR decomposition in order to reduce the order of computational complexity in detection from three

\footnotetext{
${ }^{1}$ The QR decomposition of a matrix can be calculated using the Gram-Schmidt method.
} 
to two. We refer to the QR-based MMSE BLAST detection algorithm as MMSE-SIC.

For the MMSE-SIC detector we set the spatial filter $\mathbf{W}$ to be $\mathbf{W}=\mathbf{I}_{N_{r}}$, which means that $\mathbf{y}=\mathbf{r}$ and $\mathbf{G}=\mathbf{H F}$. The MMSE-SIC detector filters the received samples using an interference nulling matrix $\mathbf{D}$ to create decision variables. The $i^{\text {th }}$ decision variable is computed using

$$
z_{i}=\mathbf{d}_{i}\left(\mathbf{y}-\sum_{j=1, j \neq i}^{L} \mathbf{g}_{j} \hat{s}_{j}\right)
$$

where $\mathbf{d}_{i}$ is the $i^{\text {th }}$ row vector of $\mathbf{D}$; called a nulling vector. To find the nulling matrix, D, the MMSE-SIC algorithm follows the QR-SIC algorithm used to find a spatial filter, with one exception: it considers the noise variance in the QR decomposition. The first step for computing $\mathbf{D}$ is to generate an augmented matrix, $\mathbf{G}_{a}$, based upon the equivalent channel matrix, G. The augmented channel matrix is created to have the following structure

$$
\mathbf{G}_{a}=\left[\begin{array}{c}
\mathbf{G} \\
\sqrt{N_{0}} \mathbf{I}_{N_{t}}
\end{array}\right]
$$

where $\mathbf{G}_{a}$ is a $\left(N_{t}+N_{r}\right) \times N_{t}$ matrix. This allows for a solution that is matched to not only the channel matrix coefficients, but also the noise vector. The next step in the MMSE-SIC algorithm is to perform a QR decomposition on the augmented channel matrix

$$
\begin{aligned}
\mathbf{G}_{a} & =\mathbf{Q}_{G_{a}} \mathbf{R}_{G_{a}} \\
& =\left[\begin{array}{c}
\mathbf{Q}_{G_{a}}^{u} \\
\mathbf{Q}_{G_{a}}^{l}
\end{array}\right] \mathbf{R}_{G_{a}}
\end{aligned}
$$

where $\mathrm{Q}_{G_{a}}^{u}$ is a $N_{r} \times\left(N_{r}+N_{t}\right)$ semi-unitary matrix; $\mathbf{Q}_{G_{a}}^{l}$ is a $N_{t} \times\left(N_{r}+N_{t}\right)$ semiunitary matrix; and $\mathbf{R}_{a}$ is a $\left(N_{t}+N_{r}\right) \times N_{t}$ upper triangular matrix. Please note that 
$\mathrm{Q}_{G_{a}}^{u} \neq \mathrm{Q}$. It was shown in [19] that by setting the nulling vectors $\mathbf{d}_{i}$ to be

$$
\mathbf{d}_{i}=\frac{1}{R_{G_{a}}(i, i)}\left[\mathbf{Q}_{G a}\left(1: N_{r}, i\right)\right]^{+}
$$

gives the same MMSE filters as would be computed using traditional approach for calculating MMSE nulling vectors. Note that here we have used MATLAB notation to represent the rows and columns to select from in each matrix.

Using the MMSE-SIC spatial filter we produce decision variables using (2.13). Unlike QR-SIC, MMSE-SIC has MAI to cancel from all transmitted symbols (besides the desired symbol), as the MMSE-SIC nulling matrix produces residual MAI for symbols $\{1, \ldots, i-1\}$. However, it should be noted that as the SNR gets larger, this MAI interference disappears because the augmented QR decomposition of the MMSE-SIC detector produces nulling vectors that are asymptotically equal to the QR-SIC spatial filter row vectors at high SNR.

\subsection{MIMO Transceivers with a Fully Informed Transmitter}

With the availability of CSI at the transmitter, the jointly designed MIMO transceiver promises throughput and BER performance advantages over the uninformed transmitter (UT) MIMO system [1]. Of course, these advantages come at the cost of additional transmitter complexity; however, the addition of a more complex transmitter can download some of the system complexity from the receiver. If designed properly, the joint transceiver can reduce the overall system complexity while still approaching the theoretical capacity of the MIMO system. This is accomplished in $[9,20,21]$. Even though it is more feasible to implement MIMO systems with only partial CSI available at the transmitter, it is still instructive to study systems where 
we have an informed transmitted (IT) that is fully aware of the channel in order to get a benchmark for performance. In this section we examine MIMO systems which are fully aware of the channel conditions at both the transmitter and receiver to design the precoder matrix, F, jointly with the spatial filter W. Systems with partial CSI at the transmitter will are investigated in subsequent chapters.

\subsubsection{Capacity Achieving Design: Singular Value Decompo- sition with the Water-Filling Algorithm}

In [9] a solution is presented that achieves the theoretical unconstrained capacity of the IT MIMO system. It takes the singular value decomposition (SVD) of the channel matrix and decomposes it into a set of $K$ parallel subchannels; where $K$ is the rank of matrix $\mathbf{H}$. As a result $L \leq K$ QAM symbols are transmitted with each channel use. The precoder, $\mathbf{F}$, is composed of a $N_{t} \times N_{t}$ semi-unitary precoding matrix, $\Omega_{1}$, and a $N_{t} \times L$ diagonal power allocation matrix, $\boldsymbol{\Phi}$; where $\mathbf{F}=\Omega_{1} \boldsymbol{\Phi}$. The power allocation matrix has the form

$$
\boldsymbol{\Phi}=\left[\begin{array}{cccc}
\phi_{1} & 0 & \cdots & 0 \\
0 & \phi_{2} & \ddots & 0 \\
\vdots & & \ddots & \vdots \\
0 & 0 & \cdots & \phi_{L} \\
0 & 0 & \cdots & 0 \\
\vdots & \vdots & & \vdots \\
0 & 0 & \cdots & 0
\end{array}\right]
$$


where $\phi_{i}$ is the power allocation to the $i^{\text {th }}$ subchannel. For each subchannel a power and rate is allocated based upon the singular values of the channel matrix and the power constraint of the transmitter. This solution assumes that the system has unconstrained rate and a method to specifically produce the rate assigned by the WFA.

The SVD of the channel matrix is

$$
\mathbf{H}=\mathbf{U} \Lambda^{\frac{1}{2}} \mathbf{V}^{+}
$$

where $\mathbf{U}$ is $N_{r} \times N_{r}$ a unitary matrix; $\boldsymbol{\Lambda}$ is a $N_{r} \times N_{t}$ diagonal matrix with each nonzero entry being an eigenvalue of $\mathbf{H}$; and $\mathbf{V}$ is a $N_{t} \times N_{t}$ unitary matrix. If we set $\mathbf{W}=\mathbf{U}^{+}, \Omega_{1}=\mathrm{V}$, then the equivalent matrix, $\mathbf{G}$, becomes $\mathbf{G}=(\boldsymbol{\Lambda} \Phi)^{\frac{1}{2}}$, which is a diagonal matrix with diagonal elements $g_{i, i}=\sigma_{i} \sqrt{\phi_{i}}$; where $\left\{\sigma_{i}\right\}$ are the singular values of $\mathbf{H}$. As a result of the diagonal nature of $\mathbf{G}$, the detector inputs are

$$
y_{i}=\sigma_{i} \sqrt{\phi_{i}} s_{i}+\nu_{i}
$$

Since there is no MAI, the detector need not do anything, so its outputs are $z_{i}=y_{i}$. A power $\phi_{i}$ is assigned to each subchannel using the WFA, so

$$
\phi_{i}=\max \left(\mu-\frac{1}{\rho_{i}}, 0\right)
$$

where

$$
\mu=\frac{1}{L}\left(\sum_{i=1}^{L} \frac{1}{\rho_{i}}+N_{t}\right)
$$

and

$$
\rho_{i}=\sigma_{i}^{2} \frac{E_{s}}{N_{0}}
$$

where $\rho_{i}$ is the signal-to-interference and noise ratio (SINR) of subchannel $i$. Note that the power allocation is normalized so that $\sum_{i=1}^{L} \phi_{i}=N_{t}$. The bit-rate, $\kappa_{i}$, to use 
on the $i^{\text {th }}$ subchannel is

$$
\kappa_{i}=\log _{2}\left(1+\phi_{i} \rho_{i}\right)
$$

This transceiver is referred to as SVD-WFA in this thesis. An advantage of the SVD-WFA transceiver is in the reduced computational complexity of its detector. Since all symbols can be detected independently without having to worry about the effects of MAI, the detector's computational complexity is low. This is because in addition to the linear computational complexity in symbol-wise detection, SIC is not required. This removes the need for the hard- or soft- symbol estimation methods needed in the QR-SIC and MMSE-SIC detectors. However, the added complexity of

computing the SVD of a matrix is $O\left(N_{t}^{3}\right)$, which has a higher degree of complexity than the computation of QR-SIC and MMSE-SIC. This means that the computation of the SVD-WFA spatial filter and precoder have higher complexity than those of QR-SIC and MMSE-SIC.

\subsubsection{The Geometric Mean Decomposition: Equal Gain Sub- channels}

The geometric mean decomposition (GMD) was first proposed by Jiang et. al. [22] in 2005 and suggested for use as a MIMO transceiver in [20]. It is a matrix decomposition that is based upon the SVD of a matrix and an additional set of Givens rotations. As a result its computational complexity is not much more than that of a SVD (i.e. it has the same degree of computational complexity).

The GMD of channel matrix $\mathbf{H}$ can be expressed

$$
\mathbf{H}=\mathbf{Q R P}^{+}
$$


where $\mathbf{Q}$ is a $N_{r} \times N_{r}$ unitary matrix; $\mathbf{R}$ is a $N_{r} \times K$ upper triangular matrix with all diagonal entries being equal to the geometric mean of $\mathbf{H} ; \mathbf{P}$ is a $N_{t} \times K$ semi-unitary matrix; and where $K$ is the rank of the channel matrix, $\mathbf{H}$. The upper triangular matrix $\mathbf{R}$ has the following structure

$$
\mathbf{R}=\left[\begin{array}{cccc} 
& & & \\
\bar{\sigma} & r_{1,2} & \cdots & r_{1, K} \\
0 & \bar{\sigma} & \ddots & r_{2, K} \\
\vdots & & \ddots & \vdots \\
0 & \cdots & 0 & \bar{\sigma}
\end{array}\right]
$$

where $\bar{\sigma}$ is the geometric mean of the singular values of $\mathbf{H}$, given by

$$
\bar{\sigma}=\sqrt[K]{\prod_{i=1}^{K} \sigma_{i}}
$$

GMD-SIC Setting the spatial filter $\mathbf{W}=\mathbf{Q}^{+}$and $\boldsymbol{\Omega}_{\mathbf{1}}=\mathbf{P}$ we obtain $\mathbf{G}=\mathbf{R}$ as our equivalent channel matrix. We note that the equivalent matrix, being upper triangular, allows us to use the SIC detection method. That is, we use (2.11) and (2.12) to obtain symbol estimates for any transmitted symbols. We refer to this transceiver as GMD-SIC. The GMD-SIC transceiver achieves receive diversity, a result derived by Jiang et. al. in [21].

The power allocation matrix for the GMD-SIC transceiver of [20] is set to send the same power on all $K$ subchannels. That is,

$$
\phi_{i}=\frac{N_{t}}{K}
$$


The GMD-SIC of [20] also asks that all subchannels send the same constellation. This is because the GMD is designed to reduce some of the transmitter complexity added by the SVD-WFA transceiver such as power and bit-rate allocation [20].

\subsubsection{Antenna Selection Geometric Mean Decomposition}

In addition to the GMD-SIC transceiver an algorithm is also provided in [20] that uses antenna selection to select the subset, $L$, from the $K$ subchannels that will achieve the highest unconstrained throughput; we denote this technique ASGMD. The ASGMD technique can be deployed using the same SIC detector as the GMD. Assuming that the singular values of $\mathbf{H}$ are ordered from largest to smallest, the ASGMD-SIC algorithm uses

$$
L=\arg \max _{L \in[1, K]} L \log _{2}\left(1+\frac{\bar{\sigma}_{L}^{2} N_{t} E_{s}}{L N_{0}}\right)
$$

subchannels, where

$$
\bar{\sigma}_{L}=\sqrt[L]{\prod_{i=1}^{L} \sigma_{i}}
$$

That is, we find the number of subchannels to use in the GMD algorithm such that the instantaneous capacity is maximized for a GMD on the subset of subchannels.

\subsubsection{Uniform Channel Decomposition: A Capacity Achiev- ing Solution with Equal SINR Subchannels}

The UCD was proposed by Jiang et. al. [21] as method of attaining the optimal capacity while using the same rate on all subchannels. This is accomplished by creating a set of subchannels that have the same receive SINR. The UCD method proposes a combination of techniques that have already been described in this chapter. It makes use of SVD, WFA, GMD and MMSE filtering to achieve the 
maximum attainable rate [21].

The UCD algorithm requires that the precoder be composed of two unitary matrices $\left(\Omega_{1}, \Omega_{2}\right)$ and a power allocation matrix $(\Phi)$, so that $\mathbf{F}=\Omega_{1} \Phi^{\frac{1}{2}} \Omega_{2}$. The spatial filter is based upon the QR-based MMSE filtering presented in Section 2.2. A mathematical description of UCD follows.

The UCD algorithm is designed such that $L \geq K$ can be used in transmitting a symbol block. In this thesis we prefer to set $L=K$ when implementing the UCD algorithm. This fact is reflected in our presentation of the UCD algorithm. The channel matrix $\mathbf{H}$ has a SVD based on (2.17), namely $\mathbf{H}=\mathbf{U} \Lambda^{\frac{1}{2}} \mathbf{V}^{+}$. Thus, we set the first precoder matrix $\Omega_{1}$ to equal the right-hand unitary matrix of the singular value decomposition of $\mathbf{H}$. Using the WFA, a power allocation matrix is found for $\boldsymbol{\Phi}$ based upon (2.19). The precoder is then

$$
\mathbf{F}=\mathbf{V} \Phi^{\frac{1}{2}} \Omega_{2}
$$

The resulting pre-coded channel matrix, which results from the matrix multiplication of the channel matrix and precoder is

$$
\Gamma=\mathbf{H F}=\mathrm{U}(\Lambda \Phi)^{\frac{1}{2}} \Omega_{2}=\mathrm{U} \Sigma \Omega_{2}
$$

where $\boldsymbol{\Sigma}=(\boldsymbol{\Lambda} \Phi)^{\frac{1}{2}}$. We then compute the augmented matrix of $\boldsymbol{\Gamma}$

$$
\Gamma_{\mathbf{a}}=\left[\begin{array}{c}
\mathrm{U} \Sigma \Omega_{2} \\
\sqrt{N_{0}} \mathbf{I}_{K}
\end{array}\right]
$$

where we know that applying a QR decomposition to this matrix yields an augmented upper triangular matrix. Further, according to Jiang et. al. in [21] for any augmented matrix as given in (2.27) there exists an $\Omega_{2}$ such that the diagonal entries of the 
augmented upper triangular will be equal. We can rewrite $\Gamma_{a}$ as

$$
\boldsymbol{\Gamma}_{\mathbf{a}}=\left[\begin{array}{cc}
\mathbf{I}_{N_{r}} & 0 \\
0 & \Omega_{2}^{+}
\end{array}\right]\left[\begin{array}{c}
\mathrm{U} \boldsymbol{\Sigma} \\
\sqrt{N_{0}} \mathbf{I}_{K}
\end{array}\right] \mathbf{\Omega}_{2}
$$

using the unitary property of $\Omega_{2}$. From (2.23) we are able to express the second matrix in (2.28) in terms of its GMD

$$
\mathbf{J}=\left[\begin{array}{c}
\mathrm{U} \Sigma \\
\sqrt{N_{0}} \mathbf{I}_{N_{t}}
\end{array}\right]=\mathbf{Q}_{J} \mathbf{R}_{J} \mathbf{P}_{J}^{+}
$$

If we set the second precoder matrix to equal the right-hand unitary matrix from the GMD of $\mathbf{J}\left(\boldsymbol{\Omega}_{2}=\mathbf{P}_{J}\right)$ and substitute the result of (2.29) into (2.28), the following result is obtained

$$
\boldsymbol{\Gamma}_{\mathbf{a}}=\left[\begin{array}{cc}
\mathbf{I}_{N_{r}} & 0 \\
0 & \Omega_{2}^{+}
\end{array}\right] \mathbf{Q}_{J} \mathbf{R}_{J}=\mathbf{Q}_{\Gamma_{a}} \mathbf{R}_{J}
$$

which resembles the result for the MMSE-SIC augmented channel matrix. In fact, the spatial filter, $\mathbf{W}$, is computed using the same nulling matrix as is defined in (2.16), but substituting $\mathbf{Q}_{\Gamma a}$ for $\mathbf{Q}_{G_{a}}$. So the row vectors of the spatial filter are computed as

$$
\mathbf{w}_{i}=\frac{1}{\mathbf{R}_{\Gamma_{a}}(i, i)}\left[\mathbf{Q}_{\Gamma_{a}}\left(1: N_{r}, i\right)\right]^{+} .
$$

A reduced complexity algorithm for UCD is given in Appendix A.1. Following the seven steps outlined there a UCD can be computed at the same computational complexity degree as the SVD and GMD algorithms, since it is based upon them.

\subsubsection{GMD-MMSE}

In [20] Jiang et. al. suggests implementing the GMD transceiver with a SIC BLAST detector, which can be represented by the QR-SIC detector of Section 2.2.3. This is 
the GMD-SIC transceiver described in Section 2.3.2. To our knowledge the GMD transceiver has not been implemented with any other detector. In this section we propose further improvement to the design of the GMD-based MIMO system by applying an additional MMSE filter at the receiver. We call this transceiver GMD-MMSE.

GMD-MMSE is implemented by calculating the MMSE nulling matrix, $\mathrm{D}$, based upon the upper triangular matrix, R, using (2.14) - (2.16).

The GMD-MMSE transceiver can be computed for little extra cost over GMD-SIC, since the MMSE nulling matrix has smaller degree computational complexity than the GMD algorithm. To compute the MMSE-SIC nulling matrix requires a degree computational complexity of two, whereas a GMD of a channel matrix requires a degree computational complexity of three. In addition, we expect the GMD-MMSE to have better throughput and BER performance than the GMD-SIC transceiver. This is because the MMSE-SIC detector is computed using more statistical information than QR-SIC. 


\section{Chapter 3}

\section{Evaluating MIMO System Performance}

In this chapter we evaluate the UT and IT transceiver models using two measures: ergodic unconstrained capacity and BER. This chapter is meant to give the reader an appreciation of the performance of the different MIMO transceivers described in Chapter 2.

\subsection{MIMO Capacity}

In this section we introduce measures for the instantaneous and ergodic capacity of a MIMO system. The ergodic unconstrained capacity of a MIMO system is used in later sections of this chapter to evaluate the performance of the transceivers described in Chapter 2.

\subsubsection{Instantaneous Unconstrained Capacity of MIMO}

Because $\mathbf{G}=\mathbf{W H F}$ the instantaneous unconstrained capacity of the MIMO channel given in (2.3) is

$$
\begin{aligned}
C & =\log _{2}\left(\frac{\operatorname{det}\left(\mathrm{E}\left[\mathbf{y} \mathbf{y}^{+}\right]\right)}{\operatorname{det}\left(\mathrm{E}\left[\boldsymbol{\nu} \boldsymbol{\nu}^{+}\right]\right)}\right) \\
& =\log _{2}\left(\frac{\operatorname{det}\left(\mathbf{I}_{N_{r}} N_{0}+\mathbf{G Q}_{\mathbf{s}} \mathbf{G}^{+}\right)}{\operatorname{det}\left(\mathbf{I}_{N_{r}} N_{0}\right)}\right)
\end{aligned}
$$


where $\mathbf{Q}_{\mathbf{s}}=\mathrm{E}\left[\mathbf{s s}^{+}\right]$. Given our assumption that each symbol component of symbol vector, $\mathbf{s}$, is selected independently, but with the same symbol energy, then

$$
\mathbf{Q}_{\mathbf{s}}=\mathbf{I}_{L} E_{s}
$$

and we can further reduce (3.1) to

$$
\begin{aligned}
C & =\log _{2}\left(\operatorname{det}\left(\mathbf{I}_{N_{r}}+\frac{E_{s}}{N_{0}} \mathbf{G G}^{+}\right)\right) \\
& =\log _{2}\left(\operatorname{det}\left(\mathbf{I}_{N_{r}}+\frac{E_{s}}{N_{0}} \boldsymbol{\Xi} \boldsymbol{\Phi} \boldsymbol{\Xi}^{+}\right)\right) \\
& =\log _{2}\left(\operatorname{det}\left(\mathbf{I}_{N_{r}}+\frac{E_{s}}{N_{0}} \boldsymbol{\Phi} \boldsymbol{\Xi} \boldsymbol{\Xi}^{+}\right)\right)
\end{aligned}
$$

where $\Xi=\mathbf{W H} \Omega_{1}$ and the above solution follows from the determinant identity property $\operatorname{det}(\mathbf{I}+\mathbf{A B})=\operatorname{det}(\mathbf{I}+\mathbf{B A})$. To further simplify (3.2) we express each spatially filtered received sample in terms of its SINR

$$
\rho_{i}=\frac{E_{s}\left|\xi_{i, i}\right|^{2} \phi_{i}}{E_{s} \sum_{j=1, j \neq i}^{L}\left|\xi_{i, j}\right|^{2} \phi_{j}+\left|\mathbf{w}_{i}\right|^{2} N_{\mathrm{o}}}
$$

where $\left\{\xi_{i, j}\right\}$ are the elements of $\Xi$, so (3.2) becomes

$$
C=\sum_{i=1}^{L} \log _{2}\left(1+\phi_{i} \rho_{i}\right)
$$

This formulation is simple, yet very instructive. Given optimal detection methods, the subchannel SINRs and power allocations fully determine the achievable unconstrained capacity of the MIMO system. The IT MIMO system's unconstrained capacity problem can be expressed as a maximization problem centered on the power allocation matrix $\Phi$

$$
C_{I T}=\sum_{i=1}^{L} \max _{\phi_{i}} \log _{2}\left(1+\phi_{i} \rho_{i}\right)
$$

subject to the total power constraint of $\sum_{i}^{L} \phi_{i}=E_{s} N_{t}$. The UT MIMO has no CSI at the transmitter so it must set its power allocation matrix to the identity matrix. 
The UT MIMO system's instantaneous unconstrained capacity is

$$
C_{U T}=\sum_{i=1}^{K} \log _{2}\left(1+\rho_{i}\right)
$$

\subsubsection{Ergodic Unconstrained Capacity of MIMO}

Earlier in this section we introduced formula for computing the instantaneous capacity of UT and IT MIMO transceivers. However, because the MIMO system is deployed in a wireless environment for which channel conditions are in constant change the instantaneous unconstrained capacity of a MIMO system is not an appropriate performance measure. A better indicator for MIMO performance is the average unconstrained capacity, measured over all possible channel realizations. This is the ergodic unconstrained capacity, given by

$$
\bar{C}=\mathrm{E}\left[\sum_{i=1}^{L} \log _{2}\left(1+\phi_{i} \rho_{i}\right)\right]
$$

where the expectation is taken over all all possible realizations of $\mathbf{H}$. For brevity we refer to the ergodic capacity as simply the capacity of a MIMO system throughout this thesis.

Figure 3.1 shows unconstrained capacity of UT and IT MIMO systems. We note that the gains made by the IT are not very significant, even at low SNRs, although the gains seen at lower SNRs are more pronounced for a larger set of antennas. In the case where CSI is not readily available to the transmitter, it must be sent back to the receiver using an alternate feedback channel. For such a small improvement in throughput at low SNRs, it may not seem worthwhile to use an IT at the cost of increased transmitter complexity. However, to attain the performance of the UT an optimal ML detector must be used. The computational complexity of the ML detector increases exponentially with the number of subchannels used, $L$, and the 


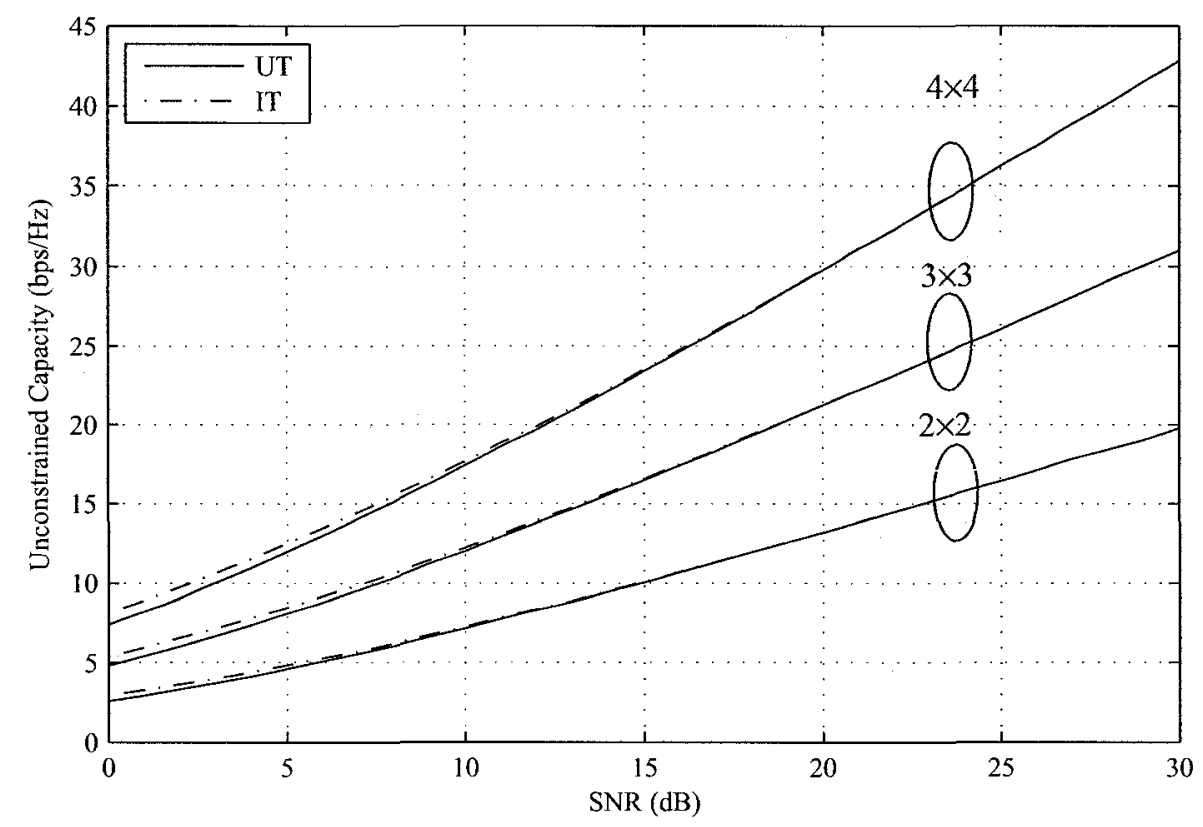

Figure 3.1: The unconstrained capacity of UT and IT MIMO systems with different numbers of receive and transmit antennas.

size of the constellations used, limiting the practical throughput achievable by the MIMO system. Since the main advantage of using MIMO is the promise of higher data rates, sub-optimal detectors have been suggested to reduce the computational complexity of the receiver of detecting each transmitted symbol. The use of a suboptimal detector will most likely lead to a loss in BER and, as a result, throughput performance. In this case it may be worthwhile to upload some complexity to the transmitter to improve the throughput performance of the MIMO system. So the problem becomes the design of a transceiver whose complexity is shared at both ends of the communication structure in order to make an implementable system that is capable of approaching the theoretical capacity. The sections that follow investigate the unconstrained capacity and BER performance of the various previously proposed UT and IT MIMO systems introduced in Chapter 2. 


\subsection{Unconstrained Capacity of MIMO Transceivers}

This section is dedicated to evaluating the unconstrained capacity of all MIMO transceivers introduced in the previous chapter. The unconstrained capacity of each transceiver is determined with the assumption that there is no error propagation, which leads to optimistic results. Thus, the capacity curves shown in this section are upper bounds for the performance of each MIMO transceiver.

\subsubsection{Unconstrained Capacity of UT Transceivers}

In this subsection we evaluate the theoretical unconstrained capacity of the UT transceivers described in Chapter 2. For all detectors any symbols that are detected in upper layers are considered to be fully cancelled by the detector when detecting the current layer. That is, we assume that there is no error propagation in detecting all layers. Figure 3.2 demonstrates the theoretical throughput advantage that the MMSE-SIC detector holds over the QR-SIC detector - especially at low SNR. The MMSE-SIC holds a $2 \mathrm{~dB}$ SNR advantage over the QR-SIC detector for the $4 \times 4$ MIMO system in achieving a spectral efficiency of $10 \mathrm{bps} / \mathrm{Hz}$. This shows that the performance improvement of MMSE-SIC over QR-SIC detection is not negligible. In addition it can be verified that the MMSE-SIC curves of Fig. 3.2 match those of the UT system from Fig. 3.1. This indicates that if error propagation is ignored, the MMSE-SIC MIMO system is capacity lossless; a result confirmed in [17]. The QRSIC curves converge with those of the MMSE-SIC detector at higher SNR because the nulling vectors of the MMSE-SIC asymptotically become those of the QR-SIC detector. These results suggest that it is worthwhile to implement the MMSE-SIC detector for the small amount of additional computational complexity that is required 


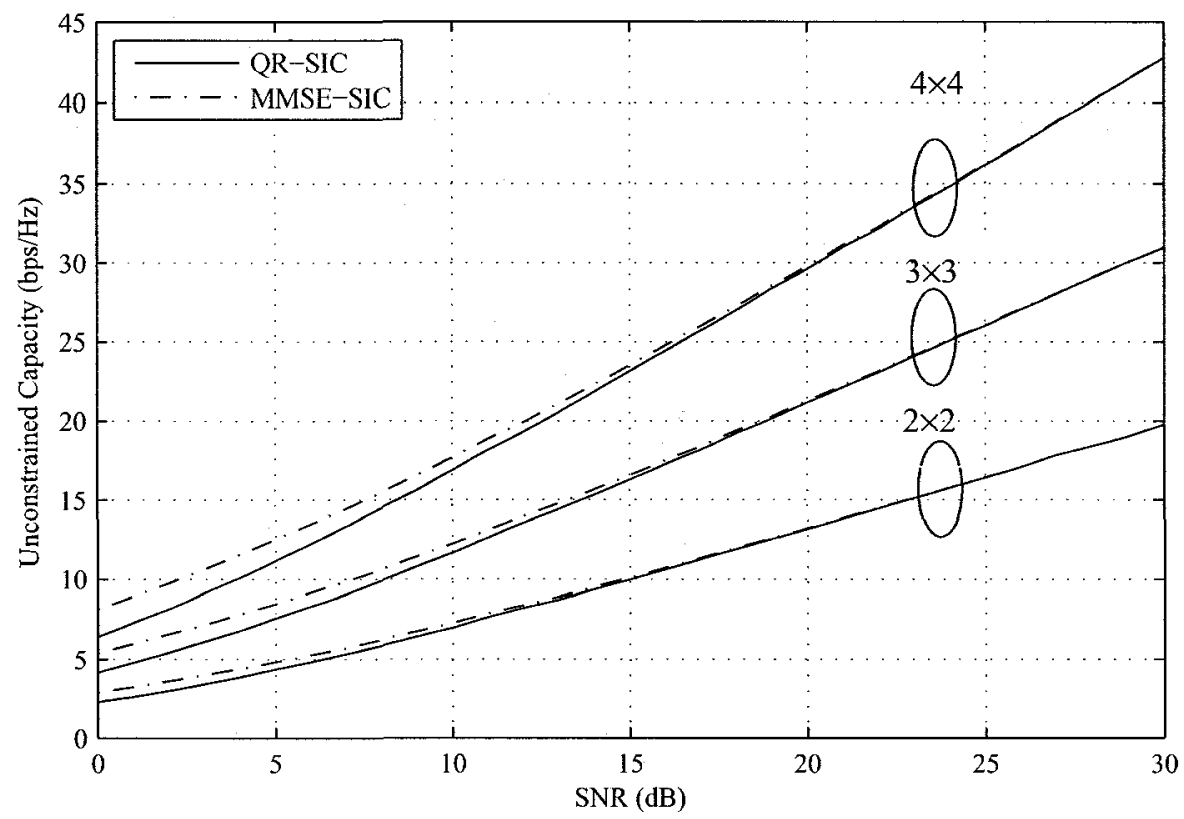

Figure 3.2: The unconstrained capacity of QR-based BLAST detectors with different numbers of receive and transmit antennas.

over an implementation of QR-SIC.

\subsubsection{Unconstrained Capacity of IT Transceivers}

The previous subsection showed the unconstrained capacity of a MIMO system with UT. This subsection is devoted to determining the unconstrained capacity of each IT MIMO system.

\section{Unconstrained Capacity of SVD-WFA}

The SVD-WFA transceiver introduced in Chapter 2 is the IT MIMO system discussed in Section 3.1. Therefore the instantaneous unconstrained capacity of SVD-WFA is

$$
C_{S V D-W F A}=\sum_{j=1}^{L} \log _{2}\left(1+\phi_{j} \rho_{j}\right) .
$$


We also know the SINR of each subchannel from Equation (2.21), allowing us to expand (3.8) to

$$
C_{S V D-W F A}=\sum_{j=1}^{L} \log _{2}\left(1+\phi_{j} \sigma_{j}^{2} \frac{E_{s}}{N_{0}}\right) .
$$

SVD-WFA would thus produce the same unconstrained capacity curves as those given for the IT MIMO system in Figure 3.1.

This linear transceiver is energy lossless as all energy pertaining to the $i^{\text {th }}$ signal is contained in the $i^{\text {th }}$ column of the resulting channel matrix $\mathrm{G}$. That is, no energy is lost in MAI cancellation or nulling. This makes the SVD-WFA transceiver capacity lossless. However, this only holds for the case where perfect CSI is available at the transmitter. For the case where the IT has only partial information (we call this a partially informed transmitter (PIT)) there are quantization errors in the precoder matrix, which will lead to MAI being contained in the decision variables and signal energy lost due to MAI cancellation and nulling operations made by the layered detector. Yet, it must be re-emphasized that for transceivers with perfect channel estimation at both ends and unconstrained spectral efficiency, this is an optimal solution.

SVD-WFA has better unconstrained capacity performance than the UT MIMO systems, at the cost of additional computational complexity at the transmitter. For an ideal IT MIMO system with perfect power and unconstrained bit-rate allocation the SVD-WFA is widely accepted as the optimal transceiver solution. However, in practice systems have design limitations, and given that SVD-WFA does not consider these limitations it is not yet clear whether it is the best transceiver to deploy for a more realistic scenario. In fact, in [20] it is claimed that the SVD-WFA is a very poor transceiver for MIMO systems limited to using the same constellation size at all transmit antennas. In this case, the WFA is said to do "inverse water-filling" [20] 
where most of the transmit power is loaded onto the most ill-conditioned subchannel at the expense of better subchannels. This "inverse water-filling" means that the SVD-WFA's performance becomes limited by the worst subchannel.

\section{Unconstrained Capacity of GMD-based Transceivers}

The instantaneous channel capacity of a GMD-SIC transceiver as presented in [20] (assuming no error propagation in the detector) is

$$
C_{G M D-S I C}=K \log \left(1+\frac{\bar{\sigma}^{2} E_{s} N_{t}}{K N_{\mathrm{o}}}\right) .
$$

The instantaneous capacity of the ASGMD-SIC transceiver where only the $L$ best subchannels are used is

$$
C_{A S G M D-S I C}=L \log _{2}\left(1+\frac{\bar{\sigma}_{L}^{2} N_{t} E_{s}}{L N_{\mathrm{o}}}\right) .
$$

Analytical solutions for the instantaneous unconstrained capacity of the GMDMMSE and ASGMD-MMSE transceivers are not easily reduced, but it is possible to find their ergodic unconstrained capacities through Monte Carlo integration. Figure 3.3 gives the upper bounds for the unconstrained capacity of the GMD-SIC and GMD-MMSE transceivers. The GMD-MMSE is shown to have a large throughput advantage over the GMD-SIC detector with a SNR gain of $1.3 \mathrm{~dB}$ over GMD-SIC at a throughput of $10 \mathrm{bps} / \mathrm{Hz}$ for the $4 \times 4 \mathrm{MIMO}$ system. It is clear that the GMD transceiver ought to be deployed with the MMSE-SIC detector. In addition, it can be verified that the unconstrained capacity curves for GMD-MMSE are the same as those for the MMSE-SIC UT MIMO system. This raises the question as to why we would need the GMD transceiver at all; however the question is deceiving. These results are for an ideal unconstrained MIMO system. The GMD transceiver is designed for MIMO systems with constrained bit-rate [20], so it would be fairer to compare the constrained capacities of these systems. 


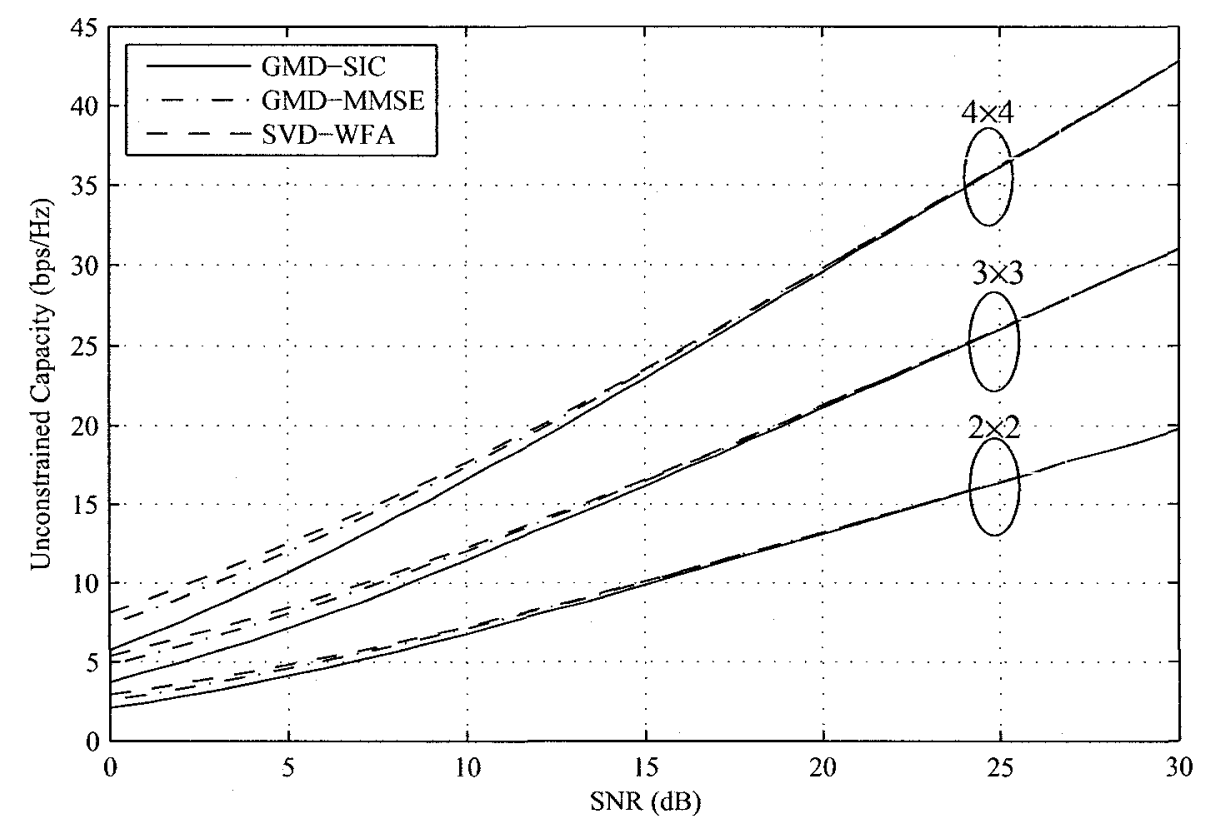

Figure 3.3: The unconstrained capacity of GMD-based BLAST detectors with different numbers of receive and transmit antennas.

Figure 3.4 shows that the ASGMD-SIC and -MMSE schemes can close the unconstrained capacity gap with SVD-WFA. It is also apparent that it does not matter whether a QR-SIC or MMSE-SIC detector is used with ASGMD, as their performances are almost exactly the same. The use of an ASGMD scheme comes at very little cost, even for a PIT. The receiver need only calculate (2.25) in order to obtain the number of subchannels, $L$, to be use in transmission and inform the transmitter. Since the receiver needs to inform the transmitter of the rank of the channel anyway, there is no additional feedback information required. Of course if we consider an IT then this calculation can be done at the transmitter as it has perfect knowledge of the channel. 


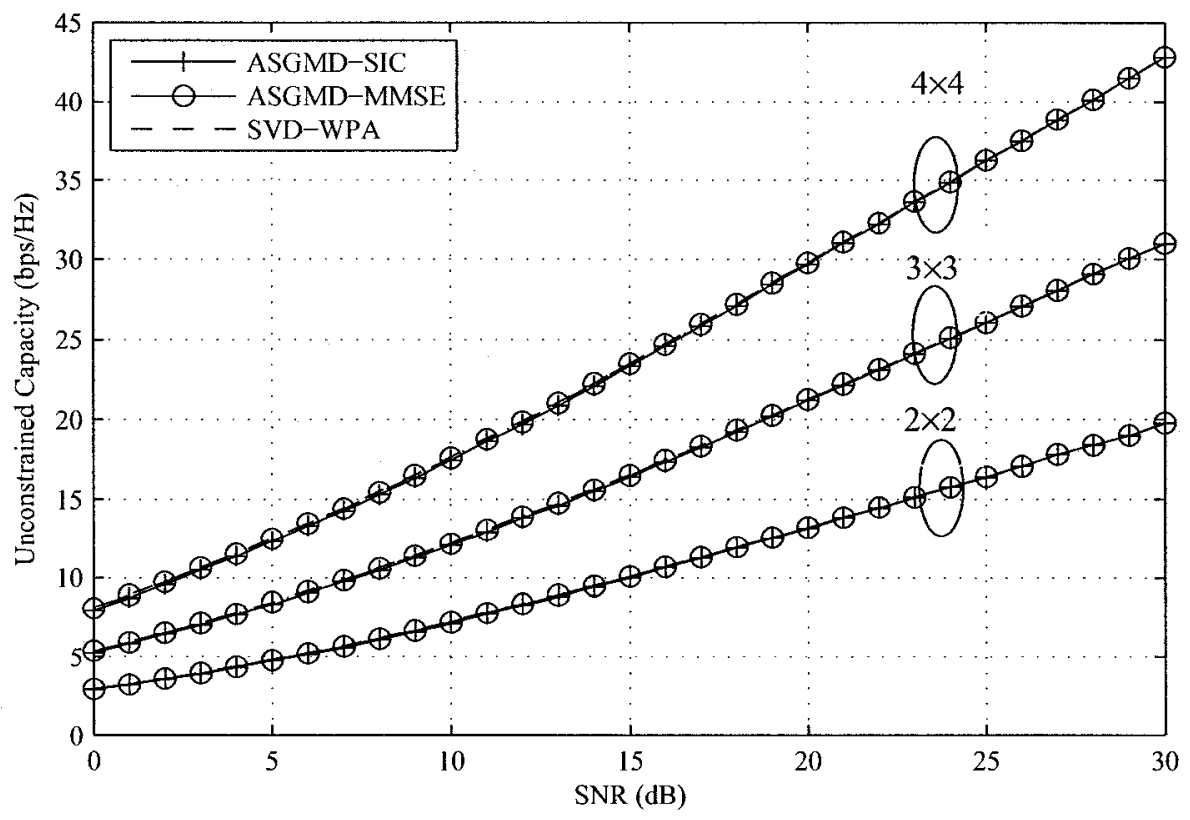

Figure 3.4: The unconstrained capacity of ASGMD-based BLAST detectors with different numbers of receive and transmit antennas.

\section{Unconstrained Capacity of UCD Transceiver}

The UCD promises to achieve the unbounded capacity of the MIMO channel with equal SINR subchannels [21]. To see if the algorithm delivers on its promise, we examine the SINR of a UCD subchannel. Using properties A.2.I and A.2.II given in Appendix A.2 the SINR of a subchannel can be expressed as

$$
\rho_{i}=\bar{\rho}=\frac{r_{J, i i}^{2}}{N_{0}}-1=\frac{\left(\prod_{j=1}^{K} \sigma_{j}^{2} \phi_{j}+N_{0}\right)^{\frac{1}{K}}}{N_{0}}-1=\left(\sqrt[K]{\prod_{j=1}^{K} \sigma_{j}^{2} \phi_{j}+1}\right)-1
$$

The instantaneous unconstrained capacity of the UCD MIMO system (given that there is no error propagation) is expressed as

$$
C_{U C D}=\sum_{j=1}^{K} \log _{2}(1+\bar{\rho})
$$


and substituting (3.12) into (3.13) gives

$$
\begin{aligned}
& C_{U C D}=\sum_{l=1}^{K} \log _{2}\left(1+\sqrt[K]{\prod_{j=1}^{K} \frac{\sigma_{j}^{2} \phi_{j}}{N_{0}}+1}\right) \\
& C_{U C D}=K \log _{2}\left(\sqrt{\prod_{j=1}^{K} \frac{\sigma_{j}^{2} \phi_{j}}{N_{o}}+1}\right) \\
& C_{U C D}=\sum_{j=1}^{K} \log _{2}\left(1+\phi_{j} \frac{\sigma_{j}^{2}}{N_{0}}\right) \\
& C_{U C D}=\sum_{j=1}^{K} \log _{2}\left(1+\phi_{j} \rho_{j}\right)
\end{aligned}
$$

which is the same capacity that is derived for the IT in Section 3.1.1 and where the SINR for each subchannel is the same as in (2.21), which is the SINR of each SVD-WFA subchannel. So the UCD is capable of creating equal SINRs on each subchannel while remaining capacity lossless, given perfect MAI cancellation.

For the SVD-WFA if all channels employed the same rate, the worst subchannel would limit the performance of the entire system [21]. From the equal SINR property, we can say that the UCD MIMO system is no longer limited by the worst subchannel.

\subsection{BER performance of the Channel Decomposi- tion Methods with Fixed Bit-Rate}

We have seen the unconstrained capacity of each IT and UT MIMO system. However, unconstrained capacity is not a great measure for a system's performance, since it is not attainable in practice. In the absence of ECC, the BER is a better measure for system performance. It gives and idea of whether or not the constrained performance of a system is good. In this section we give and discuss results for the BER performance of each MIMO system. 


\subsubsection{BER Performance of UT MIMO Systems}

In Section 2.2 we presented three detectors: ML, QR-SIC, and MMSE-SIC. The first detector has exponential and the last two have linear computational complexity. While, the ML and MMSE-SIC detectors are said to be information lossless in terms of the unconstrained capacity, they have very different BER performances. The diversity experienced by a signal is attributed to the slope of its BER curve. Typically, for a single-input single-output (SISO) transceiver with linear detection the BER curve decays proportionally to $\frac{1}{\gamma}$; or decays inverse proportionally to the SNR. With an ML detector, because the symbols are jointly detected the BER decays with a slope of $\frac{1}{(\gamma)^{N_{r}}}$. This behaviour can be attributed to the receive diversity experienced by the system. Diversity as a property of some physical attribute (time, frequency, space) is obtained by having multiple independent copies of the same information. The diversity order for linear detection without pre-coding has a tendency to be poor. This is because a linear detector sacrifices its degrees of freedom, or the number of independent received symbol copies to create a single symbol estimate. In fact, in [17] it was found that the diversity of the linear detectors presented in this thesis is $\left(N_{r}-\min \left(N_{r}, N_{t}\right)\right)+1$.

Figures $3.5-3.8$ demonstrate the BER performance of the different MIMO systems for a variety of transmitter arrays and constellation sizes. For Figures 3.5 - 3.7 all transmitters send an independently selected signal chosen from a 16-QAM constellation. Each receiver uses either a QR-SIC, MMSE-SIC or ML detector. It is easy to see that the best detector is ML, which is demonstrated in all figures. In addition to better BER performance, the ML detector's performance curves exhibit a steeper slope (indicating a higher diversity) as is promised in the previous paragraph. The slope of the QR-SIC and MMSE-SIC curves is $\frac{1}{\gamma}$. That is, these 


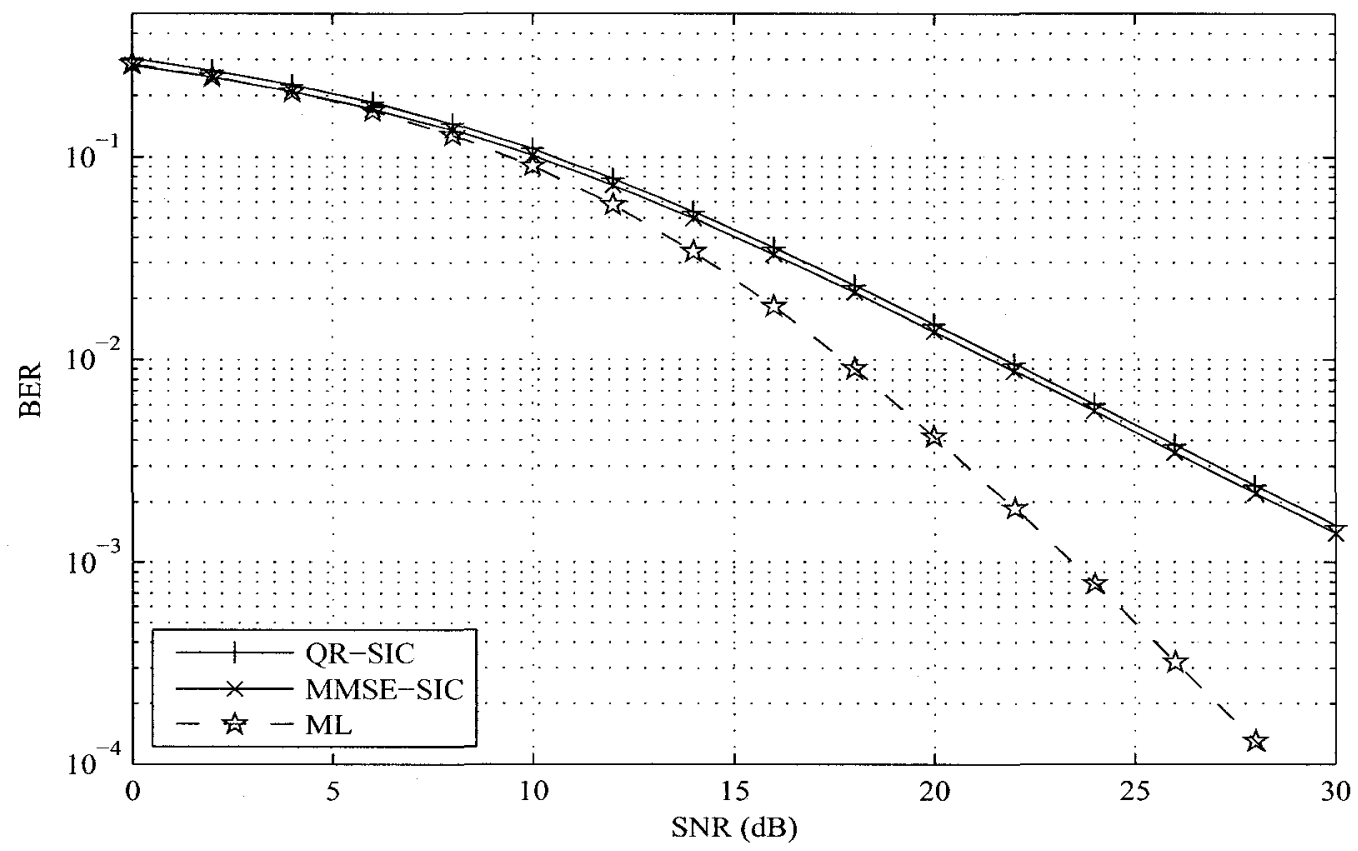

Figure 3.5: BER performance of $2 \times 2$ UT MIMO systems using 16-QAM.

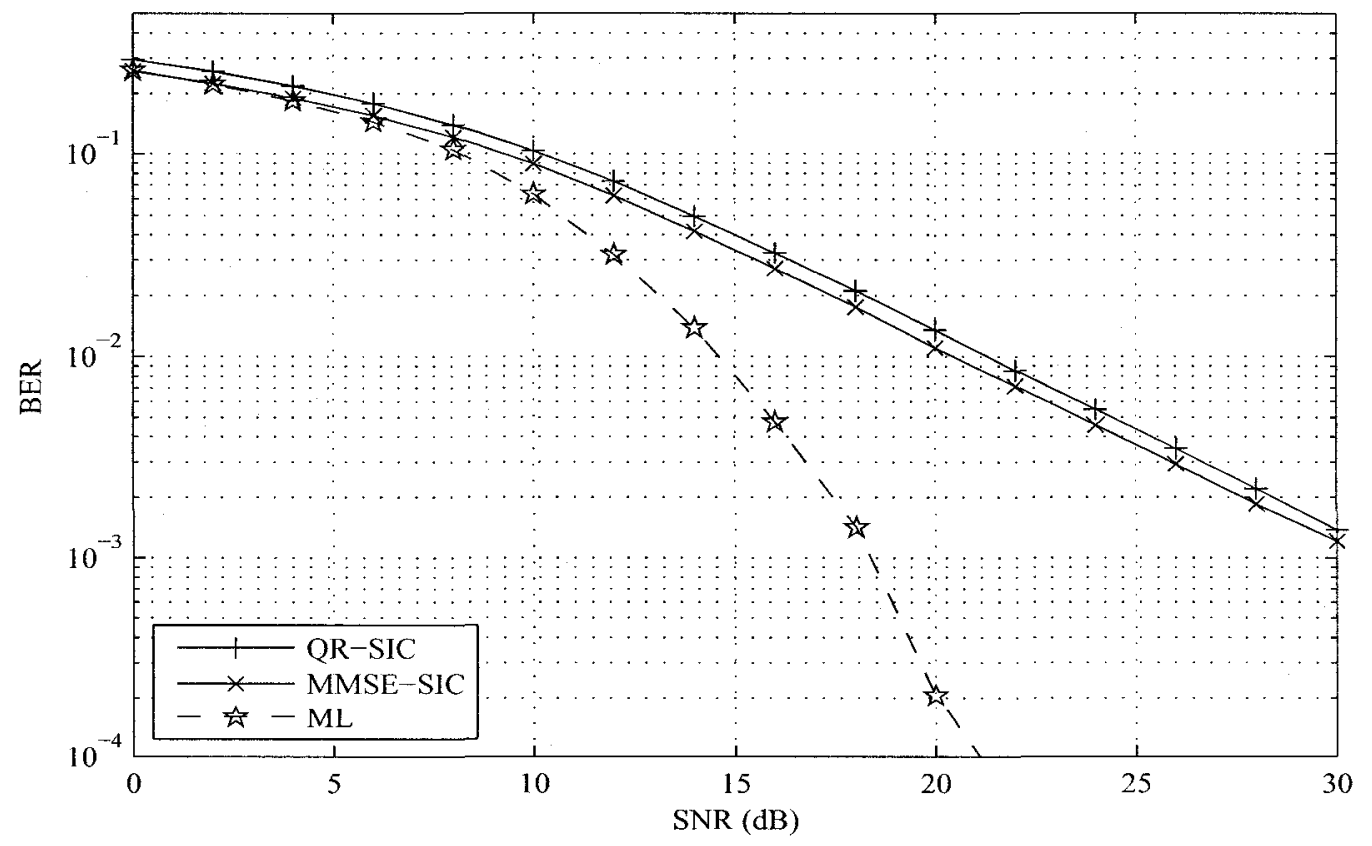

Figure 3.6: BER performance of $3 \times 3$ UT MIMO systems using 16-QAM. 


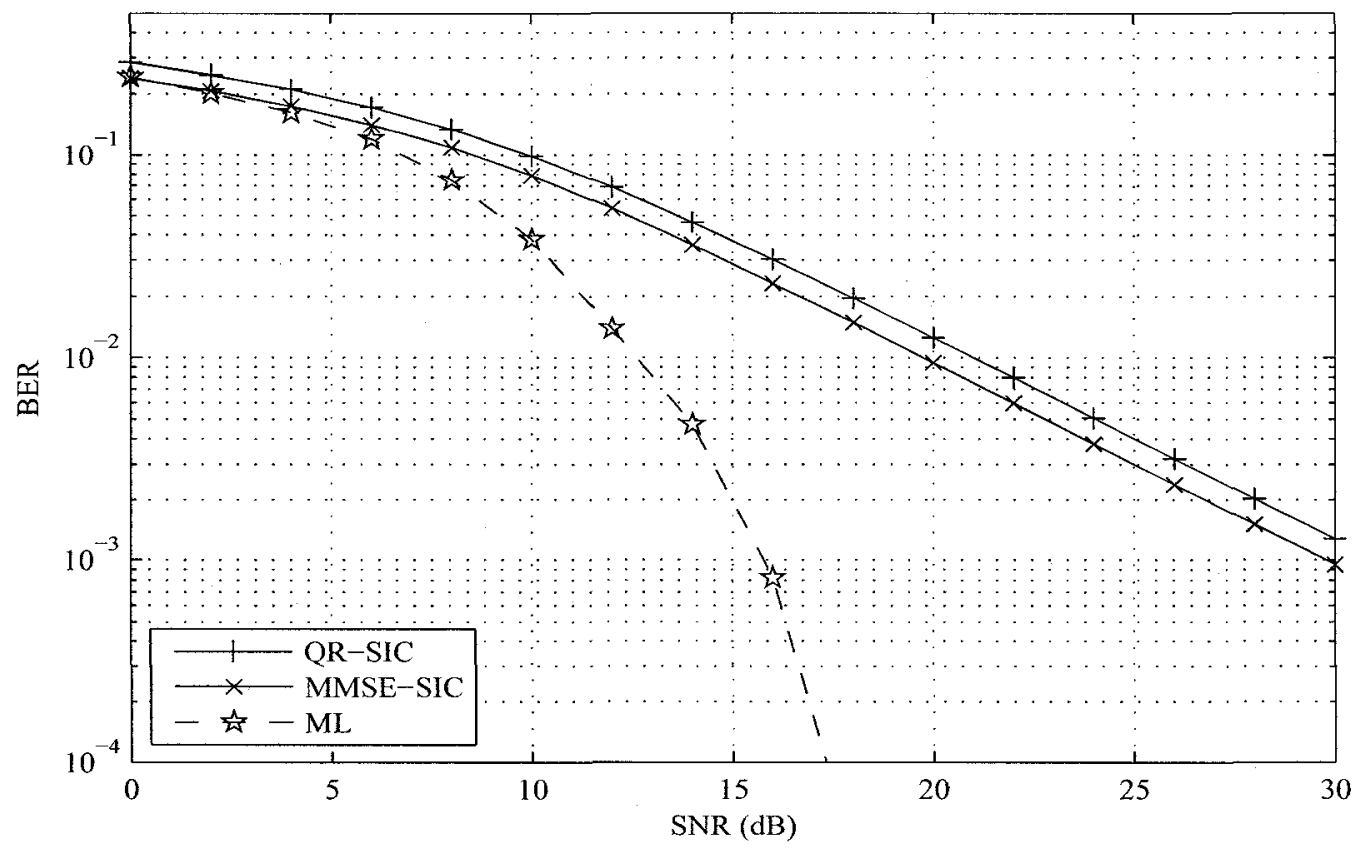

Figure 3.7: BER performance of $4 \times 4$ UT MIMO systems using 16-QAM.

detectors experience no diversity, or a diversity order of one - as is predicted in [17]. The MMSE-SIC detector outperforms the QR-SIC detector for every MIMO system employing 16-QAM at each transmitter.

Figure 3.8 shows the performance of QR-SIC and MMSE-SIC when a 64-QAM constellation is transmitted on all subchannels ${ }^{1}$. The results are similar to the case where 16-QAM is used, except for the increased BER expected for a system using higher spectral efficiencies. Though there is only a slight coding gain for the MIMO systems using larger numbers of antennas with linear detection, there is an increase in the spectral efficiency with each additional transmitter. This shows that though more data is being transmitted, there is no loss in BER performance - as long as the number of transmit and receive antenna increase equally.

\footnotetext{
${ }^{1}$ The performance of the ML detector is not shown due to the detector's high computational complexity when higher order modulation schemes are used.
} 


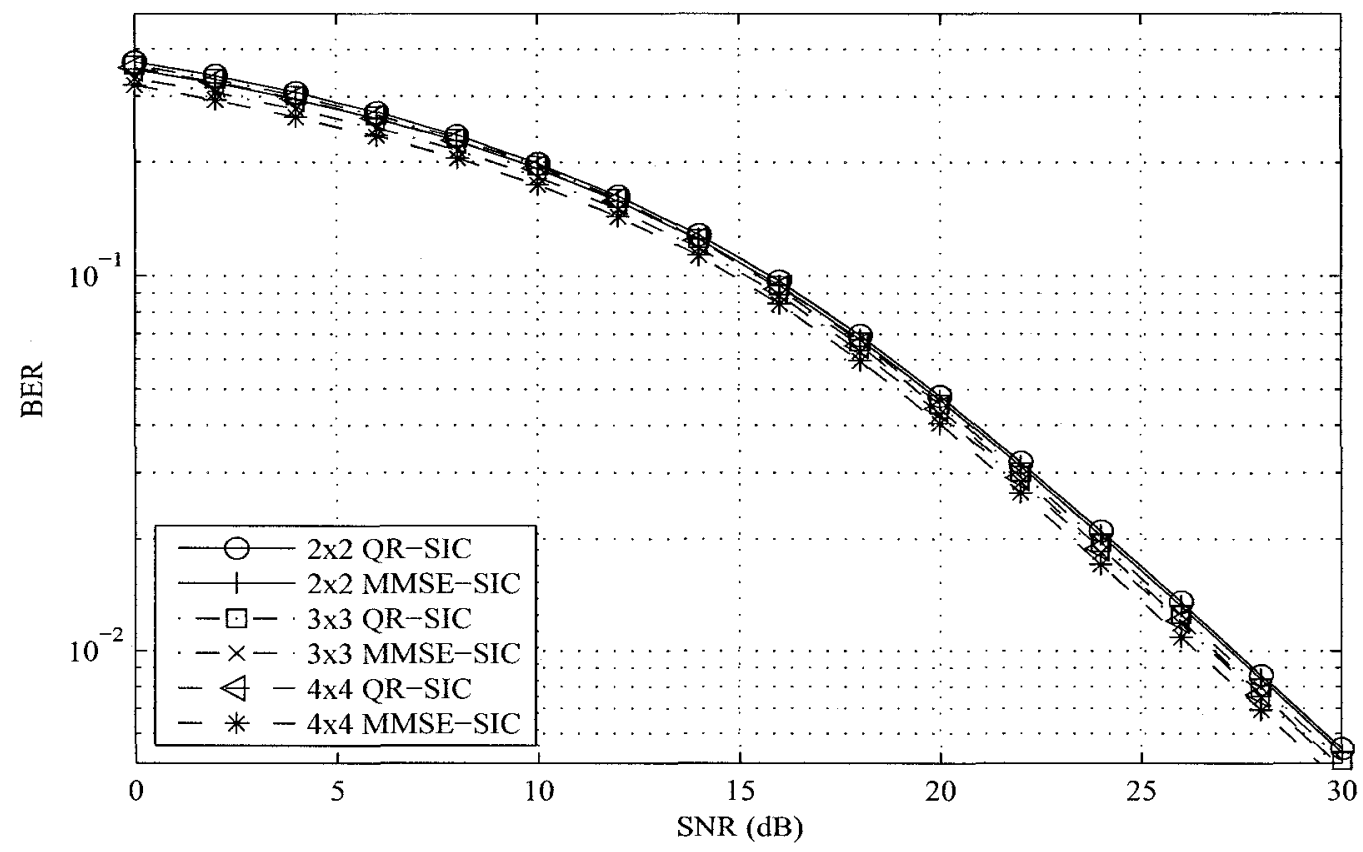

Figure 3.8: BER performance of UT MIMO systems with linear detectors using 64-QAM.

In Section 3.2.1 results calculated for the unconstrained capacity of MMSE-SIC suggested that it was information lossless, like the ML detector. Of course, the assumption that there would be no error propagation from previously detected layers was naive and we have seen this in the BER figures presented in this section. It is clear that the ML detector can achieve much better BER performance than the MMSESIC detector. Though the BER curves do not directly correlate to the constrained capacity performance of a MIMO system, they give an idea of the impact that error propagation has on layered linear detection schemes. These results demonstrate that the unconstrained capacity curves obtained in the previous section are optimistic upper bounds. 


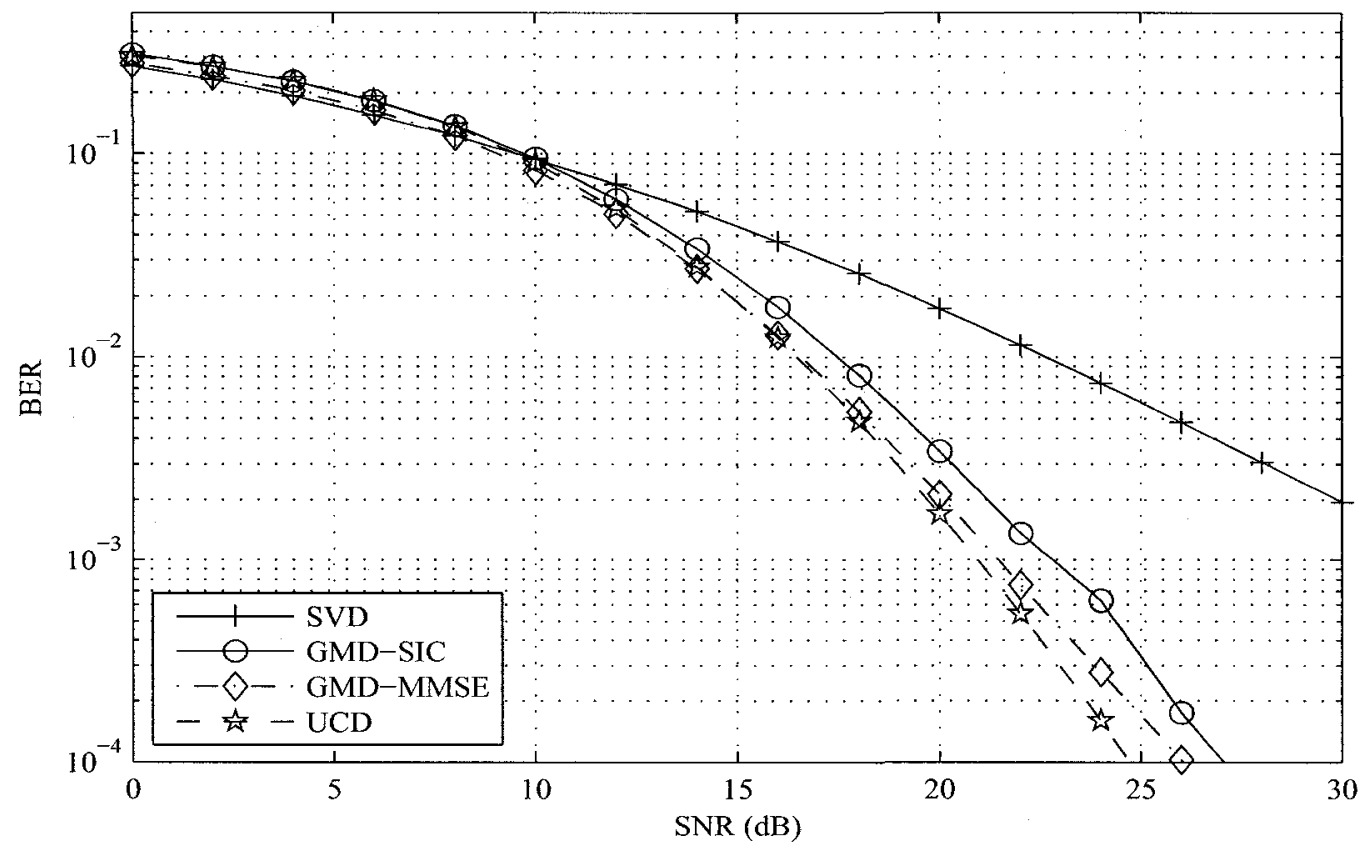

Figure 3.9: BER performance of $2 \times 2$ IT MIMO systems using 16-QAM.

\subsubsection{BER Performance of IT MIMO Systems}

In Section 2.3 we presented some channel decomposition methods: SVD, GMD and UCD. Each channel decomposition was then paired with a linear detector - either QR-SIC or MMSE-SIC. This section gives the BER performance of all detectors and their pairings. We have not included any bit-rate or power allocation for these schemes, with the exception of UCD. That is, each system has only their precoder matrices as feedback. We allow the UCD precoder to have power allocation since it would be a regular GMD otherwise.

Figures 3.9-3.11 give the performance of each IT transceiver for different numbers of antennas and with each transmitter sending a 16-QAM constellation. It is not immediately apparent that the performance of SVD-based MIMO systems are poor for the rate-constrained system. Although it has better performance than all other transceivers at low SNRs, its diversity is one and it is quickly outperformed. The 


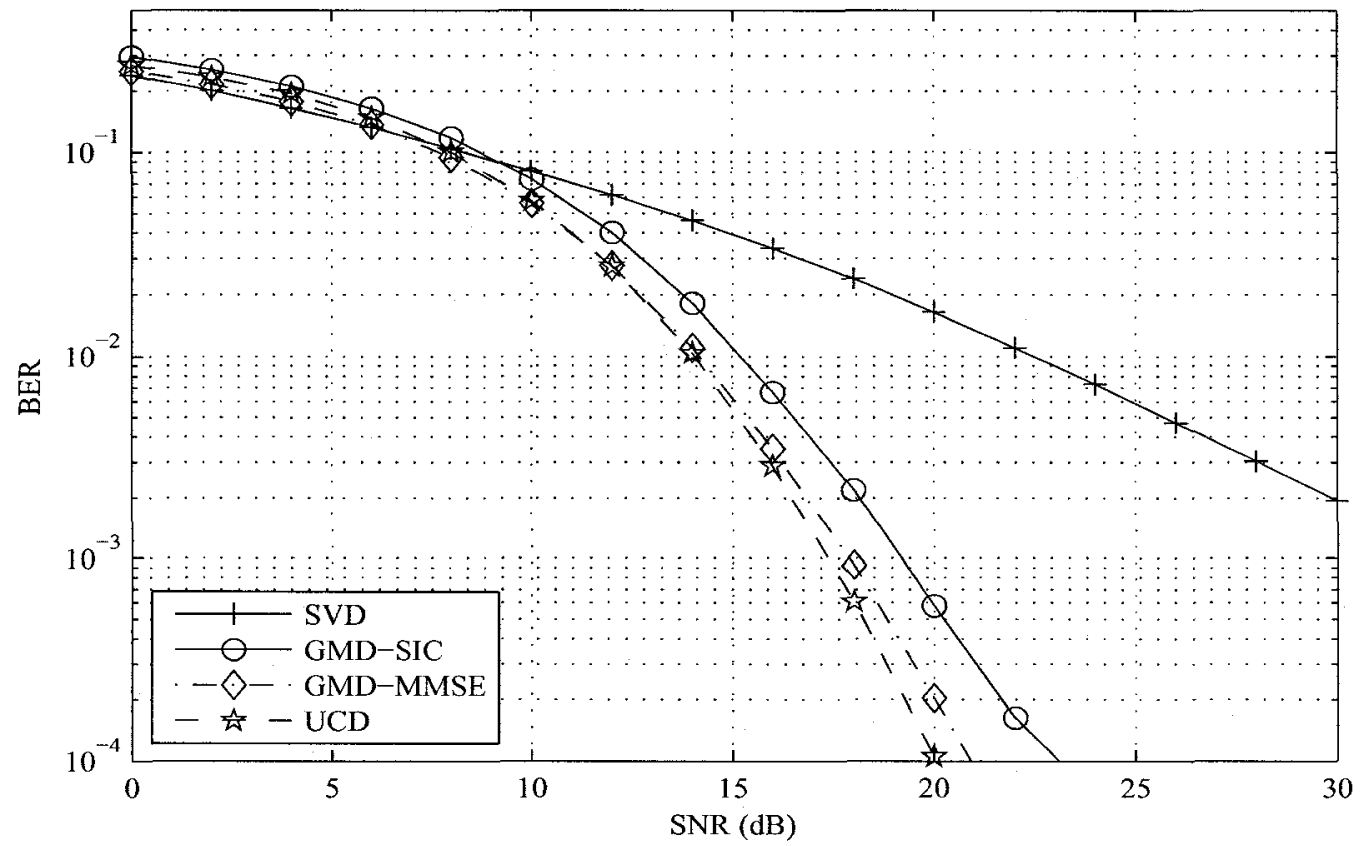

Figure 3.10: BER performance of $3 \times 3$ IT MIMO systems using 16-QAM.

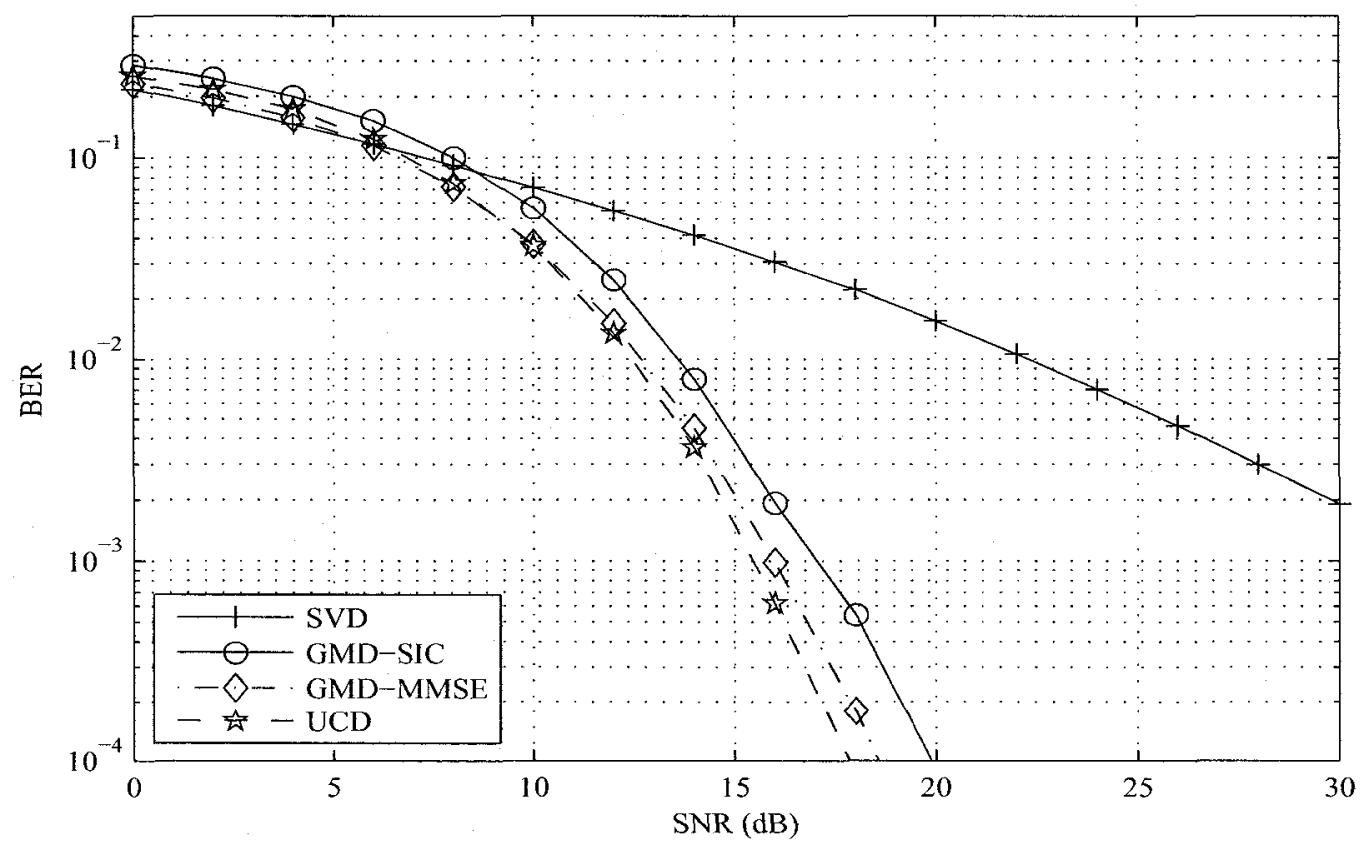

Figure 3.11: BER performance of $4 \times 4$ IT MIMO systems using 16-QAM. 
low-SNR performance gains begin to disappear for a larger number of antennas, as it can be seen that SVD is outperformed by all other transceivers at $10 \mathrm{~dB}$ for the $2 \times 2$ system; whereas it is outperformed by all other systems at about $8.5 \mathrm{~dB}$ for the $4 \times 4$ MIMO system. A GMD-MMSE implementation is better than the simple GMD-SIC detector. Figure 3.9 shows a $1 \mathrm{~dB}$ SNR gain at a BER $10^{-4}$ for the GMD-MMSE over the GMD-SIC. Figure 3.10 shows a $1.25 \mathrm{~dB}$ and Figure 3.11 a $1.5 \mathrm{~dB}$ gain at the same BER. This suggests that the gains of the MMSE method increase as the number of antennas increase. In [21] the diversity order of GMD-SIC and UCD is derived. The diversity order of GMD-SIC is determined to be $N_{r}$ and that of UCD is $N_{r} N_{t}$ - or full diversity. Figures $3.9-3.11$ demonstrate that derivations of [21] are accurate. That is, the diversity of GMD-SIC and UCD in Figure 3.9 are 2 and 4; in Figure 3.10 are 3 and 9; and in Figure 3.11 are 4 and 16 respectively. It should also be noted that UCD is outperformed by GMD-MMSE at lower SNRs. This property will be relevant in later chapters when we evaluate the constrained throughput of each system.

Figures 3.12 - 3.14 give the performance of IT transceivers for different antenna array sizes when a 64-QAM constellation is transmitted on all active subchannels. The behaviour that these figures exhibit is the same as that in Figures $3.9-3.11$, described in the previous paragraph. Most notable is the underperformance of the UCD transceiver when compared with GMD-MMSE. Though UCD has better unconstrained capacity, it is evident that with the MMSE-SIC detector, GMD has better BER at lower SNRs for MIMO systems with constrained rate. This is most evident in Figure 3.12 where GMD-MMSE outperforms UCD until the SNR is $29 \mathrm{~dB}$. This suggests that UCD is not as capable at removing MAI as GMD-MMSE in the presence of significant noise. As the number of antennas increases UCD begins to outperform 


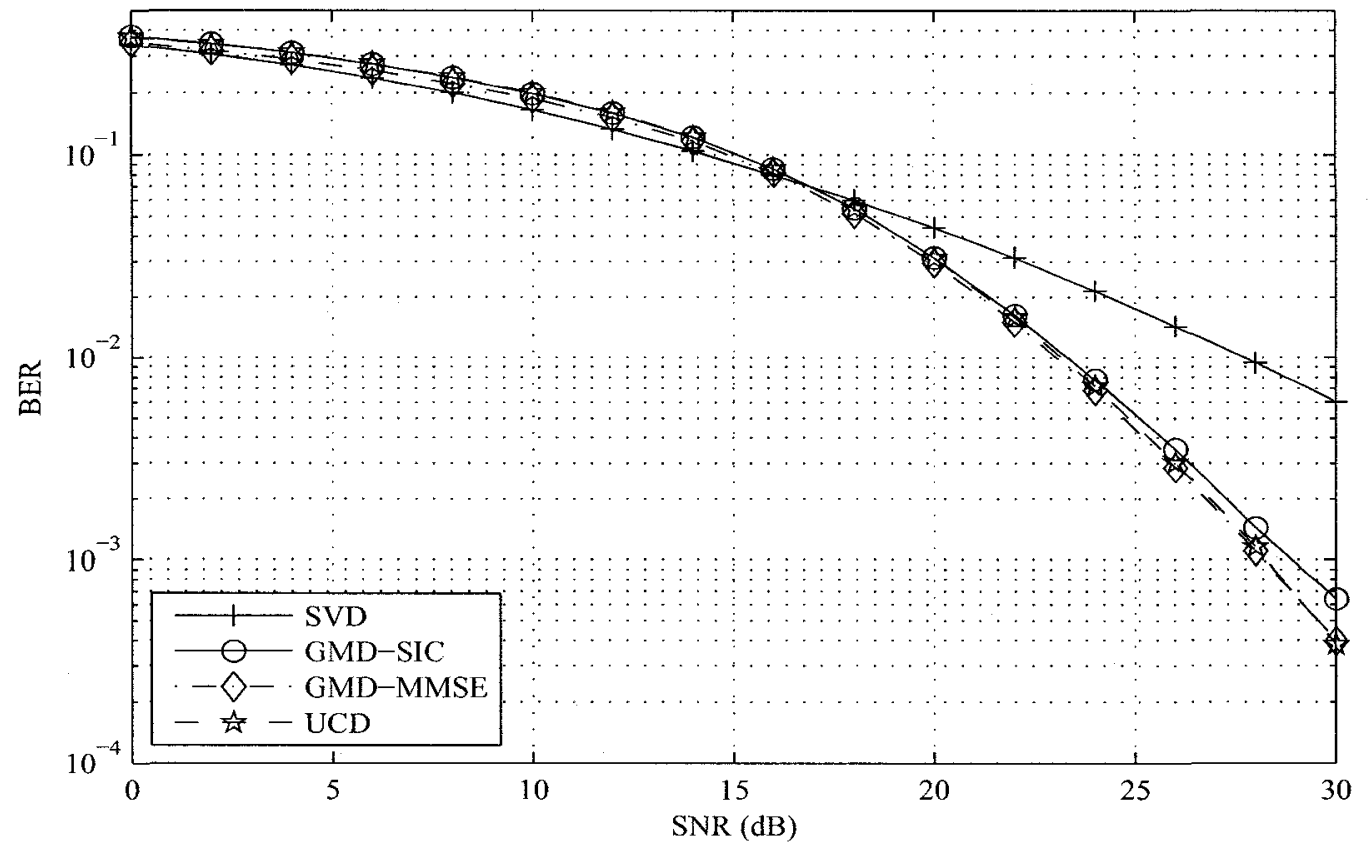

Figure 3.12: BER performance of $2 \times 2$ IT MIMO systems using 64-QAM.

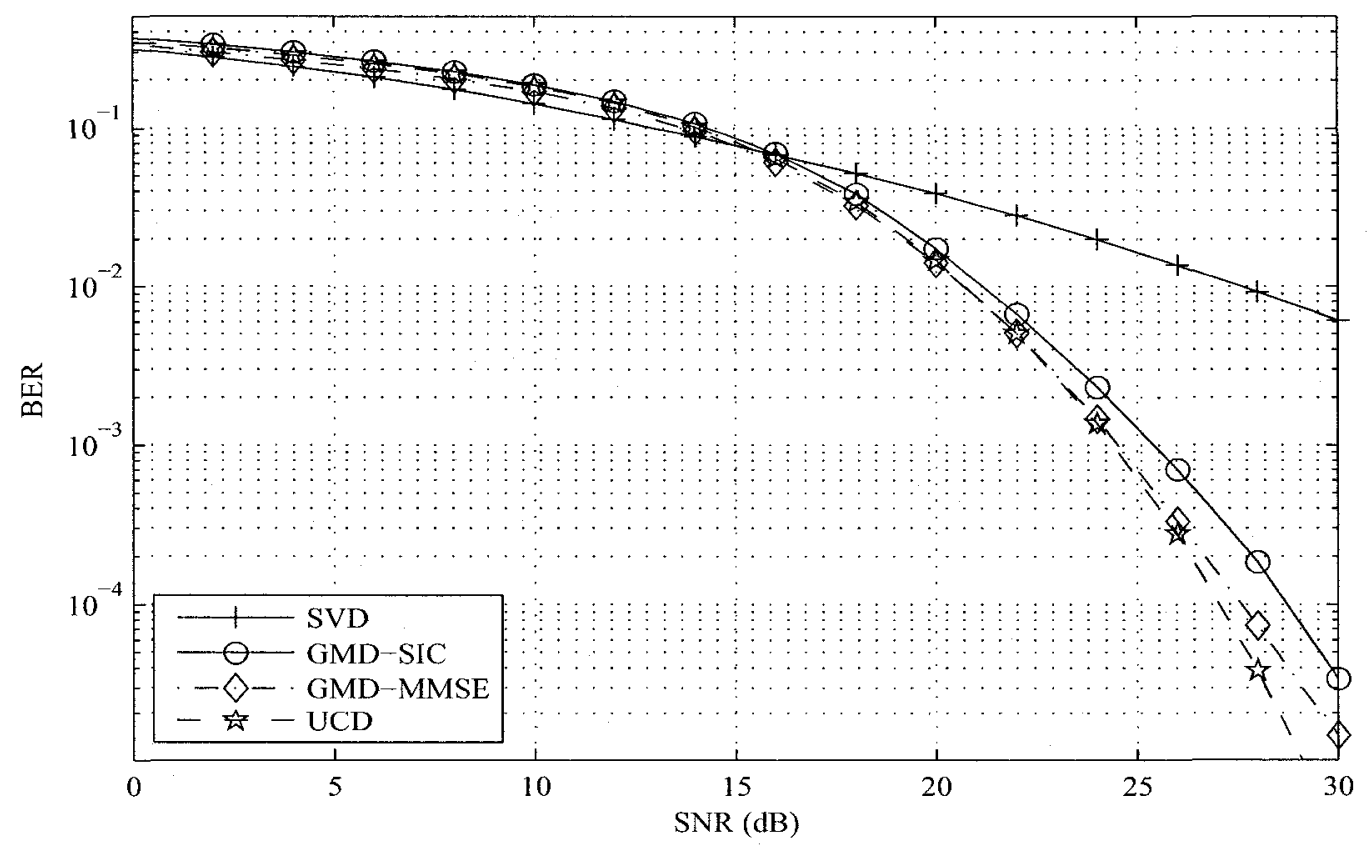

Figure 3.13: BER performance of $3 \times 3$ IT MIMO systems using 64-QAM. 


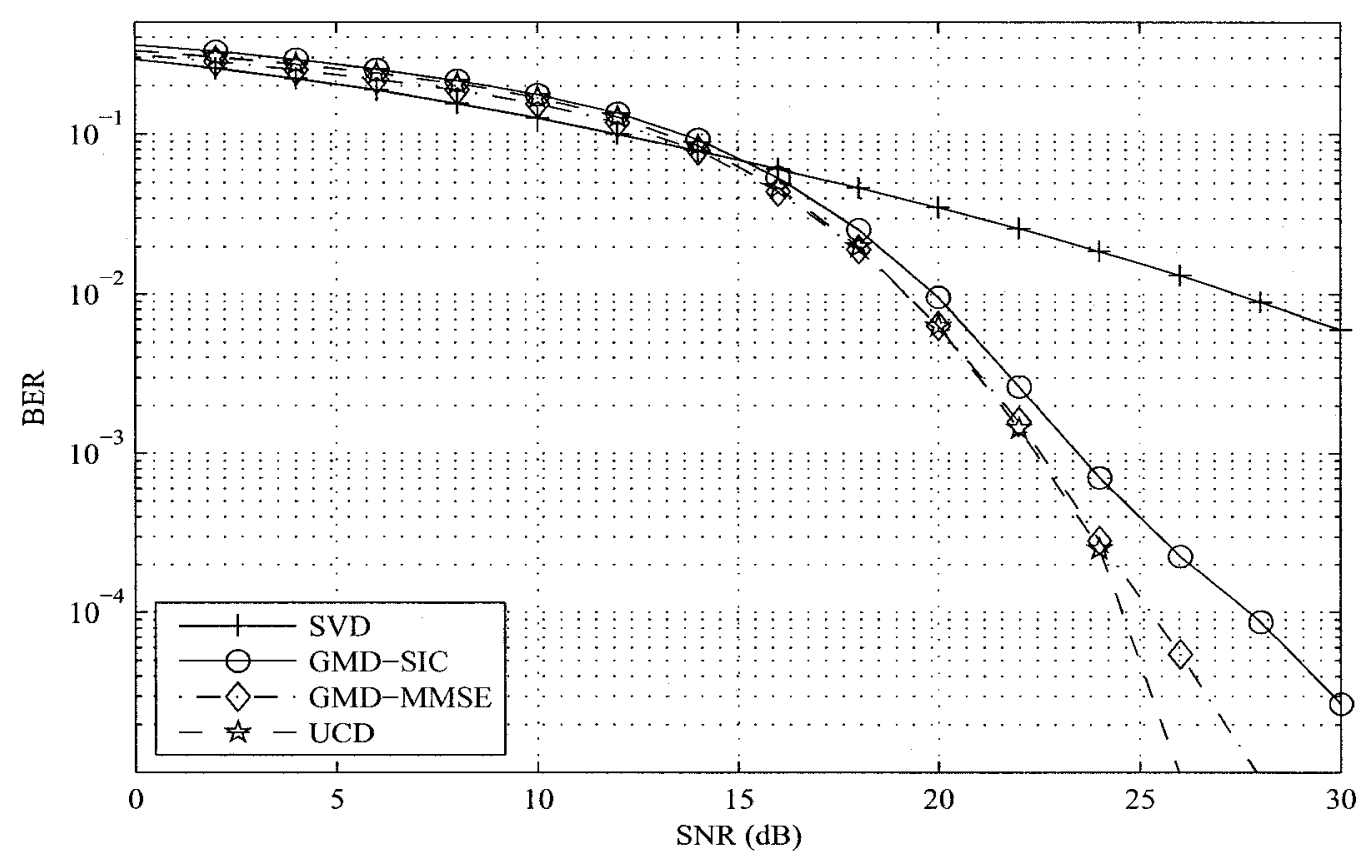

Figure 3.14: BER performance of $4 \times 4$ IT MIMO systems using 64-QAM.

GMD-MMSE at lower and lower SNRs; however we will see later that the poor lowSNR performance of UCD will have a large impact on its constrained throughput. In Figure 3.12 we see that the SVD now outperforms all other IT transceivers until roughly $17 \mathrm{~dB}$. In Figures 3.13 and 3.14 this number reduces to $16 \mathrm{~dB}$ and $15 \mathrm{~dB}$ respectively. It is an important observation since it implies that for larger constellation sizes and lower SNRs SVD is better. The main advantage that the SVD transceiver has over all others is that it experiences no MAI. Therefore, each subchannel can be detected independently of all others. This suggests that as the constellation size employed increases so does the error propagation in the SIC detectors. Of course, this stands to reason since the error probability of any individual symbol will be higher for larger constellation sizes. 


\title{
Part II
}

\section{Design of a Discrete}

\section{Rate-Constrained Coded MIMO}

\author{
System
}




\section{Chapter 4}

\section{Discrete Rate-Constrained MIMO System Design}

In this chapter we discuss how the use of RC/QC LDPC codes allow for a MIMO system that is capable of adjusting to instantaneous channel conditions. We also discuss the design and implementation issues that come with the addition of $\mathrm{RC} / \mathrm{QC}$ LDC codes to the MIMO system and evaluate the throughput performance of our designed coded RC/QC-MIMO system.

\subsection{System Model}

In Part I we presented a variety of MIMO systems and compared them in terms of their unconstrained capacity and BER performance. While these are good indicators for the achievable performance of a MIMO system they ignore some of critical practical considerations. In particular, they assume the transmitter can transmit at any rate within a continuous range, and furthermore that this range is unbounded at the upper end. In practice, the transmitter can only select from a discrete set of rates even if an adaptive coding and modulation scheme is used. The resolution of this set impacts throughput performance in that the throughput diminishes as the 
granularity becomes coarser. Furthermore, any implementation will have a largest usable constellation size, thereby putting a cap on the maximum possible rate. Both of these issues not only limit throughput to be below the unconstrained capacity, they also affect the design of the transceiver.

To provide fine resolution in the set of achievable rates it is desirable to support a large number of ECCs, each with a different code rate. Although it is possible to find good codes for any desired rate, a code that has been optimized for a given rate is likely to be unrelated to a code that has been optimized for a different rate. As a result, supporting a large number of optimized fixed-rate channel codes is likely to be infeasible, as the memory requirements to store the code definitions would be enormous, particularly if powerful codes such as LDPC codes are used. A preferable, albeit likely suboptimal approach is to use a $\mathrm{RC}$ code, where a wide range of rates can be realized by either puncturing or extending a single fixed rate mother code.

One type of RC LDPC code is the RC/QC LDPC code proposed by the 3GPP2 as a forward error correcting code for the forward data channel, forward superposed data channel and reverse OFDMA data channel [15]. They are also proposed for implementation in the IEEE 802.20 (Mobile Broadband Wireless Access) standard [23]. In this thesis we implement 3GPP2 RC/QC LDPC codes to study MIMO system performance in rate-constrained MIMO systems.

Figure 4.1 gives the system model for a MIMO system that implements RC/QC LDPC codes; a system that we refer to as RC/QC-MIMO. The precoder and spatial filter are modelled by the transceivers described in Chapter 2. The rest of the components of the RC/QC-MIMO system are described at length in the remainder of this section. 


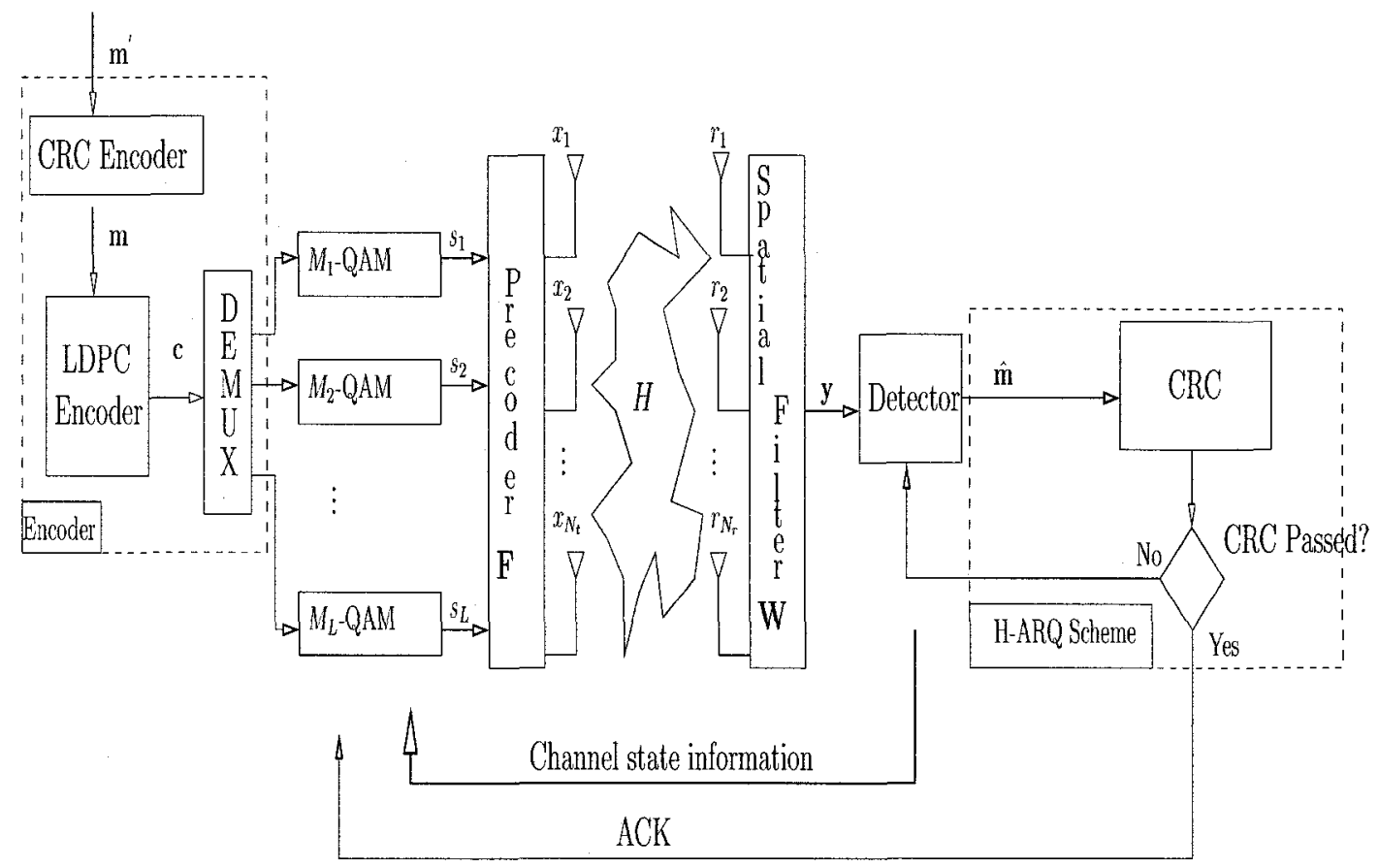

Figure 4.1: Coded MIMO system model.

\subsubsection{Encoder}

The encoder of a message word, $\mathbf{m}^{\prime}$, of length $K_{c}^{\prime}$ bits has three components as given in Figure 4.1: A cyclic redundancy check (CRC) encoder; a LDPC encoder; and a codeword demultiplexer.

\section{CRC Encoder}

The CRC encoder calculates an appropriate CRC for $\mathbf{m}^{\prime}$ and appends it to the end of this message word to create a new CRC padded message word, $\mathbf{m}$, of length $K_{c}$ bits. This CRC padding is an error-detection code (EDC) meant to determine whether or not the decoded message is valid. 


\section{LDPC Encoder}

An LDPC code is characterized by an $\left(N_{c}-K_{c}\right) \times N_{c}$ parity check matrix $\mathbf{H}_{c}$, which is the dual of the $K_{c} \times N_{c}$ generator matrix $\mathbf{G}_{c}$. These two matrices have the property that

$$
\mathbf{G}_{c} \mathbf{H}_{c}^{T}=\emptyset
$$

where $\emptyset$ is a $K_{c} \times\left(N_{c}-K_{c}\right)$ null matrix. A codeword is generated from the message word $\mathbf{m}$ using the generator matrix

$$
\mathbf{c}=\mathbf{m G}_{c}
$$

where $\mathbf{c}$ is a vector codeword of length $N_{c}$ bits. The LDPC code rate is $R_{c}=\frac{K_{c}}{N_{c}}$. For a fixed-rate LDPC code, the code- and message word lengths are fixed. However, for a $\mathrm{RC} / \mathrm{QC} \mathrm{LDPC}$ code the codeword length is variable, while the message word length remains fixed. The codeword length is adapted by puncturing some of the codeword bits, creating a shortened codeword. The codeword puncturing technique used in this thesis is described in Appendix B.

\section{Demultiplexer}

The demultiplexer splits the codeword into $L$ bit streams to be fed into the symbol mappers. The multiplexing is performed based on the sizes of the QAM constellations used by the symbol mappers. For $i$ increasing from 1 to $L$, the demultiplexer delivers $\log _{2}\left(M_{i}\right)$ bits to symbol mapper $i$. Once all the symbol mappers have received the bits they need for one channel use, the demultiplexer delivers the bits needed for the next channel use. 


\subsubsection{Symbol Mapper}

The $M$-QAM symbol mappers of Figure 4.1 have their rates specified by the CSI feedback relayed from the receiver. Normally these rates could be specified by the rateallocation portion of the WFA; however the WFA works under the assumption that the bit-rate is unconstrained. A discrete bit-rate allocation algorithm is not specified for the WFA. As a result the $M$-QAM symbol mappers are given two options: map a 64-QAM constellation or map no constellation (in the case where the transmit power of a subchannel is zero). A better constrained-rate power and bit-rate allocation algorithm is proposed and studied in Chapter 5.

\subsubsection{Detector}

Figure 4.2 gives the detector architecture, which is the iterative Turbo-BLAST detector of [11]. Adaptive soft filters are applied to $\mathbf{y}$ to null the MAI, using the most up-to-date statistical information. The MAI is estimated using soft-symbol estimators and cancelled from the filtered signals at each layer using the statistical information available at that particular layer to create decision variables, $\left\{z_{i}\right\}$. The log-likelihood ratio (LLR) of each code bit is estimated by feeding the decision variables into the bank of look-up carry units (LCUs). These LLRs are then fed into a code bit multiplexer to re-order the code bit LLRs for codeword decoding. The decoder then takes the LLRs and does iterative decoding using the belief propagation (BP) algorithm. Two outputs are produced by the BP decoder: an estimate of the systematic portion of the codeword and the new code bit LLRs that resulted at the end of the decoding attempt. The new code bit LLRs are used in updating the statistical information of each symbol. The CRC of the message word estimate, $\hat{\mathbf{m}}$, is verified to check if the received message is valid. There is a maximum number of iterations, $L_{z}$, for which the detector will run. If the maximum number of iterations in the detection process 


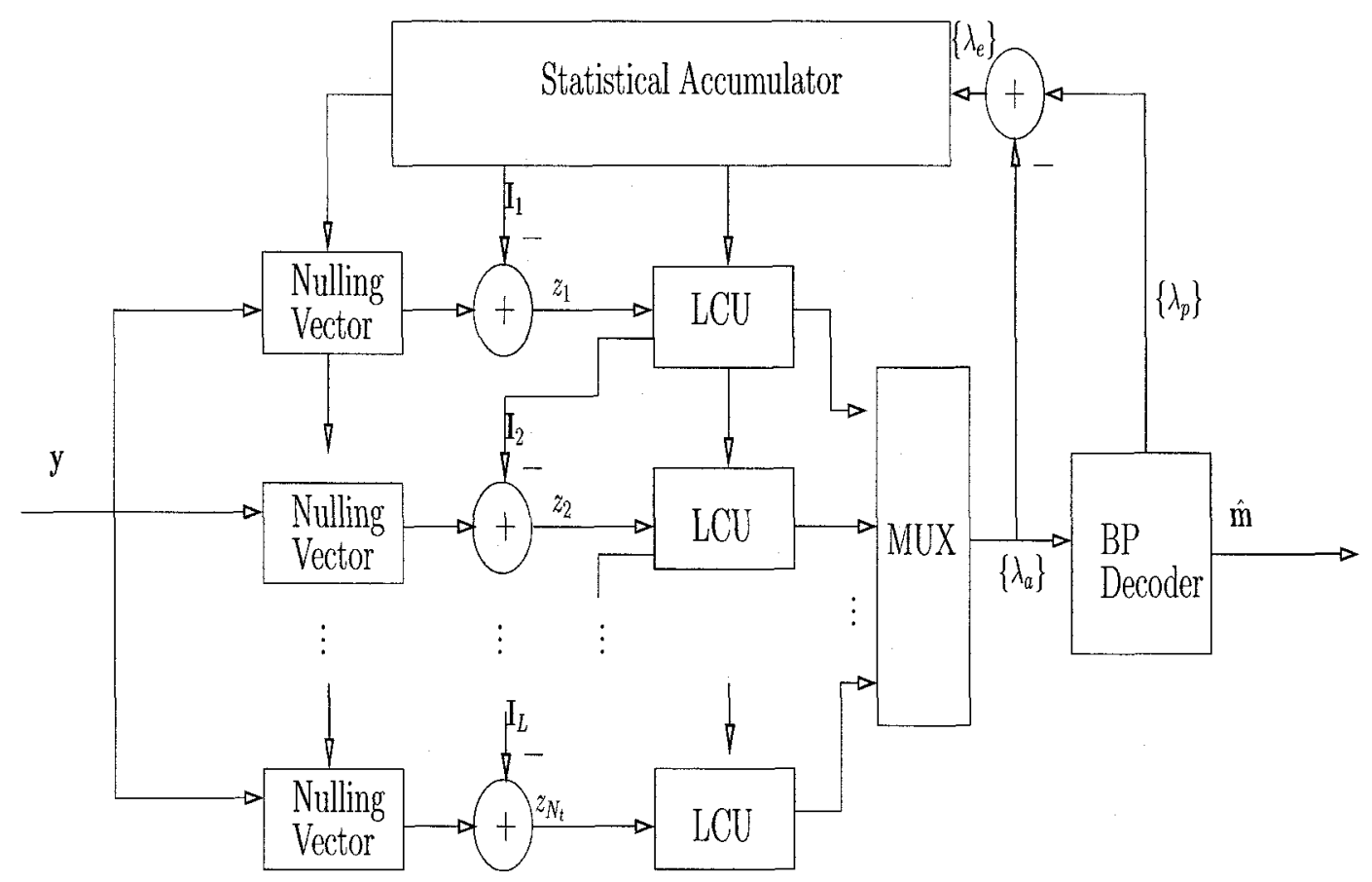

Figure 4.2: The Turbo-BLAST detector

is exceeded or the CRC on the message word estimate passes, the detection process stops.

\section{Iterative Soft Filtering}

To obtain decision variables from the received signal vector, a set of nulling vectors, $\left\{\mathbf{d}_{i}\right\}$, is required. In Chapter 2 these nulling vectors where obtained with some statistical knowledge of the noise, the assumption that no a priori information is available to the detector about the MAI and the assumption that there is no error propagation from MAI cancellation. However, because of the structure of the BLAST detection, a priori information on the MAI is available to the detector at lower detection layers in the form of a posteriori information from higher layers, and from previous detector iterations. Using this information, a soft filter - called a nulling vector - adapted to the MAI and noise statistics can be designed for each detection layer. 
To understand how the detector performs soft-symbol interference cancellation, suppose that the transmitted symbols, $\left\{s_{i}\right\}$, are transmitted with an arbitrary a priori probability distribution, $\operatorname{Pr}\left\{s_{i}=s\right\}$ for all $s \in \mathcal{S}_{i}$; where $\mathcal{S}_{i}$ is the constellation that $s_{i}$ is selected from. If no other information about $s_{i}$ were known, a hard-symbol estimate of $s_{i}$ could be

$$
\hat{s}_{i}=\arg \max _{s \in \mathcal{S}_{i}} \operatorname{Pr}\left\{s_{i}=s\right\} .
$$

In contrast, a soft-symbol estimate of $s_{i}$ could be

$$
\bar{s}_{i}=\mathrm{E}\left[s_{i}\right]=\sum_{s \in \mathcal{S}_{i}} s \operatorname{Pr}\left\{s_{i}=s\right\} .
$$

Whereas $\hat{s}_{i}$ is guaranteed to be in $\mathcal{S}_{i}, \bar{s}_{i}$ is unlikely to be. However, $\bar{s}_{i}$ has a smaller mean squared estimation error,

$$
\sigma_{s_{i}}^{2}=\mathrm{E}\left[\left|s_{i}-\bar{s}_{i}\right|^{2}\right]=\sum_{s \in \mathcal{S}_{i}}\left|s-\bar{s}_{i}\right|^{2} \operatorname{Pr}\left\{s_{i}=s\right\}
$$

than $\hat{s}_{i}$, and is therefore likely to be closer to $s_{i}$. Equations (4.3) and (4.4) are used by the detector to assist with MAI cancellation, but, in the context of iterative BLAST detection, our information regarding $s_{i}$ comes, not from the a priori probabilities, but from a posteriori probabilities calculated when detecting higher layers, or from previous decoding iterations.

To detect $s_{i}$, the detector first attempts to cancel the MAI using the soft symbol estimators, giving

$$
\begin{aligned}
\mathbf{y}_{i} & =\mathbf{y}-\sum_{j=1, j \neq i}^{L} \mathbf{g}_{j} s_{j} \\
& =\mathbf{g}_{i} s_{i}+\sum_{j=1, j \neq i}^{L} \mathrm{~g}_{j}\left(s_{j}-\bar{s}_{j}\right)+\boldsymbol{\nu} .
\end{aligned}
$$

The detector then attempts to suppress the residual MAI (the second term of the right-hand-side of (4.5) by using an interference nulling vector, $\mathbf{d}_{i}$. The detector 
output is then $z_{i}=\mathbf{d}_{i} \mathbf{y}_{i}$.

The nulling vectors are computed using the MMSE criteria. An appropriate filter for the $i^{\text {th }}$ transmitted symbol attempts to minimize the minimum squared error $\left(J_{i}\right)$ between the symbol and the detector output $z_{i}$. The MSE is

$$
\begin{aligned}
J_{i} & =\mathrm{E}\left[\left|s_{i}-z_{i}\right|^{2}\right]=\mathrm{E}\left[\left|s_{i}-\mathbf{d}_{i} \mathbf{y}_{i}\right|^{2}\right] \\
& =\mathrm{E}\left[\left|s_{i}-\mathbf{d}_{i}\left(\mathbf{g}_{i} s_{i}+\sum_{j=1, j \neq i}^{L} \mathbf{g}_{j}\left(s_{j}-\bar{s}_{j}\right)+\boldsymbol{\nu}\right)\right|^{2}\right] .
\end{aligned}
$$

Setting the partial derivative, $\partial J_{i}$, with respect to the Hermitian of nulling vector, $\mathbf{d}_{i}$, to zero gives a solution for the $i^{\text {th }}$ MMSE nulling vector

$$
\mathbf{d}_{i}=E_{s} \mathbf{g}_{i}^{+}\left(E_{s} \mathbf{g}_{i} \mathbf{g}_{i}+\sum_{j=1, j \neq i}^{L} \mathbf{g}_{j} \mathbf{g}_{j} \sigma_{s_{j}}^{2}+\mathbf{I}_{L} N_{\mathrm{o}}\right)^{-1}
$$

where $\sigma_{s_{j}}^{2}$ is the variance of the $s_{j}$ based on (4.4). This solution is adaptable to the reliability of the MAI estimates used for MAI cancellation. That is, the variances of the current estimates of the MAI, $\left\{\sigma_{s_{j}}^{2}\right\}$, are used to determine the filter coefficients. This allows the filters to be used in an iterative manner. Note that by using these soft filters, instead of hard ones, the degree of computational complexity for calculating each nulling vector increases from two to three due to the matrix inversion and multiplication required in their computation. However, the results presented in this thesis demonstrate that sizeable gains in performance are realized by using this iterative method of creating soft nulling vectors, which we call iterative soft filtering (ISF).

\section{Soft-Symbol Estimation}

The detector output, $z_{i}$, is used to generate the soft-symbol estimates used by the lower layers of the BLAST detector. These are based on the a posteriori probabilities 
of $s_{i}$

$$
\operatorname{Pr}\left\{s_{i}=s \mid z_{i}\right\}=\frac{f_{z_{i}}\left(z_{i} \mid s_{i}=s\right) \operatorname{Pr}\left\{s_{i}=s\right\}}{f_{z_{i}}\left(z_{i}\right)}=\frac{1}{K_{z}} f_{z_{i}}\left(z_{i} \mid s_{i}=s\right)
$$

where $f_{z_{i}}\left(z_{i} \mid s_{i}=s\right)$ is the likelihood function of $z_{i}$ given that $s_{i}=s$ was transmitted, and $K_{z}$ is a normalization constant chosen so that $\sum_{s \in \mathcal{S}_{i}} \operatorname{Pr}\left\{s_{i}=s \mid z_{i}\right\}$ equals one. These a posteriori probabilities are used in place of the a priori probabilities used in (4.3) and (4.4) when calculating $\bar{s}_{i}$ and $\sigma_{s_{i}}^{2}$ for detecting lower layers.

To evaluate the likelihood function of (4.8), it is widely assumed that the residual MAI after filtering can be modelled as a Gaussian random variable, so the likelihood function has the form

$$
f_{z_{i}}\left(z_{i} \mid s_{i}=s\right)=\frac{1}{\pi \sigma_{z_{i}}^{2}} \exp \left(\frac{\left|z_{i}-\mathrm{E}\left[z_{i} \mid s_{i}=s\right]\right|^{2}}{\sigma_{z_{i}}^{2}}\right)
$$

where the conditional mean of $z_{i}$ is

$$
\mathrm{E}\left[z_{i} \mid s_{i}=s\right]=\mathbf{d}_{i} \mathbf{g}_{i} s
$$

and the conditional variance is

$$
\begin{aligned}
\sigma_{z_{i}}^{2} & =\mathrm{E}\left[\left|z_{i}-\mathbf{d}_{i} \mathbf{g}_{i} s\right|^{2} \mid s_{i}=s\right] \\
& =\sum_{j=1, j \neq i}^{L}\left|\mathbf{d}_{i} \mathbf{g}_{j}\right|^{2} \sigma_{s_{j}}^{2}+\left|\mathbf{d}_{i}\right|^{2} N_{0} .
\end{aligned}
$$

\section{LLR Calculation}

A BP decoder requires LLRs for each code bit to do iterative decoding. The LCUs in Figure 4.2 compute these LLRs using the decision variables created by the detector. If BPSK is used there is a one-to-one relationship between code bits and transmitted symbols, so the LLRs are easy to compute. However, when QAM is used, calculation of the LLRs requires marginalization over the a posteriori probabilities of (4.8). Let $\mathbf{c}_{i}=\left[c_{i}^{(1)}, c_{i}^{(2)}, \ldots, c_{i}^{\left(\log _{2}\left(M_{i}\right)\right)}\right]$ denote the code bits mapped to $s_{i}$, 
and $\boldsymbol{\lambda}_{i}=\left[\lambda_{i}^{(1)}, \lambda_{i}^{(2)}, \ldots, \lambda_{i}^{\left(\log _{2}\left(M_{i}\right)\right)}\right]$ denote the corresponding LLRs. The LLRs are defined as

$$
\lambda_{i}^{(k)}=\ln \left(\frac{\operatorname{Pr}\left\{c_{i}^{(k)}=1 \mid z_{i}\right\}}{\operatorname{Pr}\left\{c_{i}^{(k)}=0 \mid z_{i}\right\}}\right)
$$

and are calculated with

$$
\lambda_{i}^{(k)}=\ln \left(\frac{\sum_{s \in \mathcal{S}_{i}} \operatorname{Pr}\left\{s_{i}=s \mid z_{i}\right\} \operatorname{Pr}\left\{c_{i}^{(k)}=1 \mid s_{i}=s\right\}}{\sum_{s \in \mathcal{S}_{i}} \operatorname{Pr}\left\{s_{i}=s \mid z_{i}\right\} \operatorname{Pr}\left\{c_{i}^{(k)}=0 \mid s_{i}=s\right\}}\right)
$$

where, for $\alpha \in\{0,1\}, \operatorname{Pr}\left\{c_{i}^{(k)}=\alpha \mid s_{i}=s\right\}=1$ if the $k^{t h}$ bit of the code bits corresponding to the point $s$ in the QAM constellation is $\alpha$, and zero otherwise. Although the computational complexity of (4.12) is quite high, the complexity can be reduced for Gray-coded QAM by splitting the real and imaginary part of $\mathcal{S}_{i}$ into two $\sqrt{M_{i}}$-PAM constellations [23]. Various suboptimal, yet effective, reduced complexity techniques have also been proposed, but these are beyond the scope of this thesis.

\section{LLR Multiplexer}

The LLR multiplexer takes the LLRs created by the LCUs and organizes them into the order that the corresponding code bits had when they were produced as a codeword. This sequence of LLRs is then passed to the decoder of the LDPC code.

\section{Belief Propagation Decoder}

Due to the structure of LDPC codes an efficient message passing algorithm (MPA), otherwise known as BP in [24] can be used to iteratively correct errors in the received LLRs. The parity-check matrix, $\mathbf{H}$, can be represented by a bipartite graph - called a Tanner graph [25] - composed of variable and parity-check nodes. The variable nodes are initialized with the LLRs produced from the decision variables and share information with the parity-check nodes that they are connected with, which can be 
viewed as the passing of a message. The parity-check nodes, having collected information from all variable nodes that they are connected with, process this information and return the result as messages to their respective variable nodes. The variable nodes then process the parity-check messages that they receive to form a new LLR. This process encompasses one iteration of $\mathrm{BP}$ decoding. With each iteration more messages are exchanged, increasing the likelihood that the algorithm will converge on the transmitted codeword. The BP decoder is allowed to run a maximum of $L_{m}$ iterations when, if convergence has not occurred, decoding is considered to have failed. The CRC code embedded in the message can be checked to verify whether or not decoding was successful.

\section{Statistical Accumulator}

If decoding is unsuccessful, the LLRs produced by the BP decoder $\left(\left\{\lambda_{p}\right\}\right)$ are used with the LLRs produced by the LCUs $\left(\left\{\lambda_{a}\right\}\right)$ to update the symbol statistics. The extrinsic LLRs

$$
\left\{\lambda_{e}\right\}=\left\{\lambda_{p}\right\}-\left\{\lambda_{a}\right\}
$$

which represent the information derived by the BP decoder, are passed back to the detector. These extrinsic LLRs are combined to produce revised a posteriori probabilities (APPs) of the transmitted symbols, $\left\{s_{i}\right\}$. These APPs are more reliable than the ones produced by the detector since they incorporate constraints imposed by the LDPC code. The APPs are used to calculate new soft symbols, $\left\{\bar{s}_{i}\right\}$, and the associated variances $\left\{\sigma_{s_{i}}^{2}\right\}$, using (4.3) and (4.4). These quantities, in turn, are used for interference cancellation until newer APPs are produced by the BLAST detector as each layer is processed. 


\subsubsection{Type-II H-ARQ}

A Type-II H-ARQ is used by our RC/QC-MIMO system. The incremental redundancy adaptive-feedback coding scheme specified in [26] is the Type-II H-ARQ. Type-II H-ARQ requires that a communication system encode message words using an EDC and a RC systematic ECC.

In Figure 4.1 the CRC represents the EDC and the RC/QC LDPC code the ECC. H-ARQ functions by first having the communication system transmit the systematic portion of a codeword (the CRC padded message). If the decision on the received message word, $\hat{\mathbf{m}}$, successfully passes the CRC then the receiver sends an acknowledgement that the message was successfully received; otherwise if the transmitter fails to receive an acknowledgment from the receiver, it proceeds to send a block of parity bits of length $L_{b}$. The receiver, with this additional information then attempts to correct the message word using at most $L_{m}$ iterations in the LDPC decoder to produces a new received message word. After each BP iteration a CRC is done on the received message word and BP stops if the CRC passes. If the CRC fails on the $L_{m}^{t h}$ iteration, the detector enters its next iteration. If, after $L_{z}$ joint iterations between the detector and the decoder, the CRC still fails, a decoding error is declared and the receiver waits for the next block of parity bits. Upon the receipt of another block of $L_{b}$ parity bits the receiver attempts to correct the message word again, using this extra information. This procedure continues until either the CRC check passes on the received message or the maximum number of parity bits for a codeword is exceeded.

The best H-ARQ system would use $L_{b}=1$ and $L_{m}=\infty$ in order to preserve rate granularity and give the best possible ECC decoding result. However, these 
parameters would be impossible to implement as the number of detector computations would be extremely large. Moreover, the delay between decoding of an ECC codeword would exceed the time to produce an acknowledgment before the transmitter begins transmitting the next block of parity bits. In this thesis we use $L_{m}=80$ and $L_{b}$ as specified in Appendix B. Furthermore, for simplicity we assume an ideal error-free CRC. That is, the CRC only ever passes if the correct message is received. The CRC code is also assumed to have a rate of one.

\subsubsection{Multiple Versus Single Codeword Demultiplexing of Data Stream}

Figure 4.1 gives an RC/QC-MIMO implemented using just one codeword - referred to as a single codeword (SCW) implementation. A SCW implementation uses one ECC code to encode the input data stream. As an alternative we could have used $L$ multiple CRC and LDPC encoders (one per used subchannel) to encode the input data stream - referred to as a multiple codeword (MCW) implementation. This implementation would require that the input data be demultiplexed into $L$ message words and encoded independently using $L$ CRC and LDPC encoders. This means that for the MCW implementation, the demultiplexer of Figure 4.1 precedes the CRC and LDPC encoders as opposed to succeeding them.

The advantage of using a MCW implementation is that the power and bit loading of one subchannel can be assumed independent of all other subchannels. The same cannot be said for a SCW implementation. One disadvantage of using a MCW implementation is operational issues that arise when coding over individual subchannels. Each subchannel will have different decoding times and this will lead either to synchronization problems, if subchannel are allowed to immediately begin transmitting 
their next codeword upon successful decoding, or throughput loss, if a successful subchannel must wait for all others subchannels to finish transmitting their messages. The disadvantages of MCW outweigh those of SCW, and as result, we use a SCW implementation in this thesis.

\subsection{Performance Evaluation of RC/QC-MIMO}

This section is dedicated to evaluating the throughput performance of the RC/QCMIMO system described in Section 4.1. We first define a metric for the throughput of the $\mathrm{RC} / \mathrm{QC}$ communication system and then evaluate system performance in an AWGN channel. The two subsections that follow examine the theoretical and experimental throughput performance of RC/QC-MIMO.

\subsubsection{The Average Realizable Rate of RC/QC-MIMO}

Using the SCW implementation an average realizable rate (ARR) is calculated to determine the throughput of the MIMO system. The ARR is calculated as the amount of time spent sending message data over the total time spent sending codeword information. In calculating the ARR a few assumptions must be made about how the system behaves in the event of a decoder failure. Classically, when a decoding failure occurs the codeword would be re-transmitted until there is successful detection. This is done without considering any of the information contained in the previous transmissions of the same codeword. This means that the realized rate $\mathrm{RR}_{j}$ of the $j^{\text {th }}$ transmission using an SCW implementation can be calculated as

$$
\mathrm{RR}_{j}=m_{T}^{(j)} \frac{K_{c}}{N_{c}^{(j)}}
$$


where $m_{T}^{(j)}$ is the total bit rate during the $j^{\text {th }}$ channel use, or given that $\left\{M_{i}^{(j)}\right\}$ are the constellations used by each subchannel during channel use $j, m_{T}^{(j)}=\sum_{i=1}^{L} \log _{2}\left(M_{i}^{(j)}\right)$; and $N_{c}^{(j)}$ is the number of transmitted code bits of the $j^{\text {th }}$ codeword. Now, if the channel is very badly conditioned, it may take several re-transmissions before the codeword is properly decoded; if it is decoded at all. Since we study a frequencyflat slow-fading channel, it is possible that the channel will have a capacity that is lower than $R_{c}^{(m i n)}$, which is define in Appendix B as the minimum rate of the RC/QC LDPC code. While it is still possible to decode a codeword that has been transmitted through a channel with extremely low gain, it is theoretically possible that it may never be decoded. That is, at least for the time it takes for the channel to change, which is unsuitably long in slow-fading systems. In our implementation of RC/QCMIMO we assume that the system will attempt to transmit through any channel. However, if a codeword is not properly decoded the user of this channel is assumed to be dropped from the network that is serving it. The impact of that this user has on the overall throughput is included only once. Otherwise, if the channel is very poor and the network continued trying to serve this user, the overall ARR may drop significantly. Based on this assumption, the ARR is computed as follows

$$
\mathrm{ARR}=\frac{K_{c} N_{S}}{\sum_{j=1}^{N_{T}} N_{c}^{(j)} \frac{1}{m_{T}^{(j)}}}=\frac{N_{S}}{\sum_{j=1}^{N_{T}} R R_{j}^{-1}}
$$

where $N_{S}$ is the number of successfully transmitted codewords and $N_{T}$ is the total number of transmitted codewords. For each transmitted codeword an independent channel realization is used. The ARR is measured in bps/Hz. 


\subsubsection{Experimental Performance of RC-QC LDPC Codes in an AWGN Channel}

We run Monte Carlo simulations for different modulation schemes using RC/QC LDPC codes in an AWGN channel and compare their throughput (ARR) performance to the theoretical constrained capacity of each modulation scheme. The constrained AWGN channel capacity when given a $M$-QAM constellation is used can be computed using numerical integration as

$$
C_{M-Q A M}=\sum_{s \in \mathcal{S}} \frac{1}{M} \int_{-\infty}^{\infty} f_{r}(r \mid s) d r
$$

where $f_{r}(r \mid s)$ is the likelihood function of $r$ being received given symbol $s$ was transmitted. Note that for $M=2$ a BPSK constellation is used. Figures $4.3-4.6$ give the throughput performance for BPSK, 4-QAM, 16-QAM and 64-QAM given a 'short', 'medium' and 'long' maximum codeword size $\left(N_{c}^{(\max )}\right)$, which are 264, 1056, 4224 code bits, corresponding to a message size of 48, 192 and 768 bits respectively. Figure 4.3 shows the throughput performance of the RC/QC LDPC codes with BPSK. There is a performance loss when compared with the theoretical capacity of BPSK since the code is not infinite or perfect. The penalty is at most $1 \mathrm{~dB}$. Figure 4.3 also shows that the shortest LDPC codes outperform the longer ones at higher SNRs. Better performance of shorter LDPC codes goes against conventional knowledge, since well-constructed long LDPC codes typically give better performance. In this case, since all the codes are built from the same generator matrix it would make more sense if the longer LDPC codes performed better than the shorter ones; however if we pay attention to the fact that an infallible rate- $1 \mathrm{CRC}$ is used to verify the correctness of the systematic portion of the codeword, these results begin to make more sense. A shorter codeword is less likely to have an error in it than a longer one before decoding. This means that the resolvability of the EDC occurs at lower SNRs for the shorter RC/QC LDPC codes. Of course, in practice, the CRC is neither 


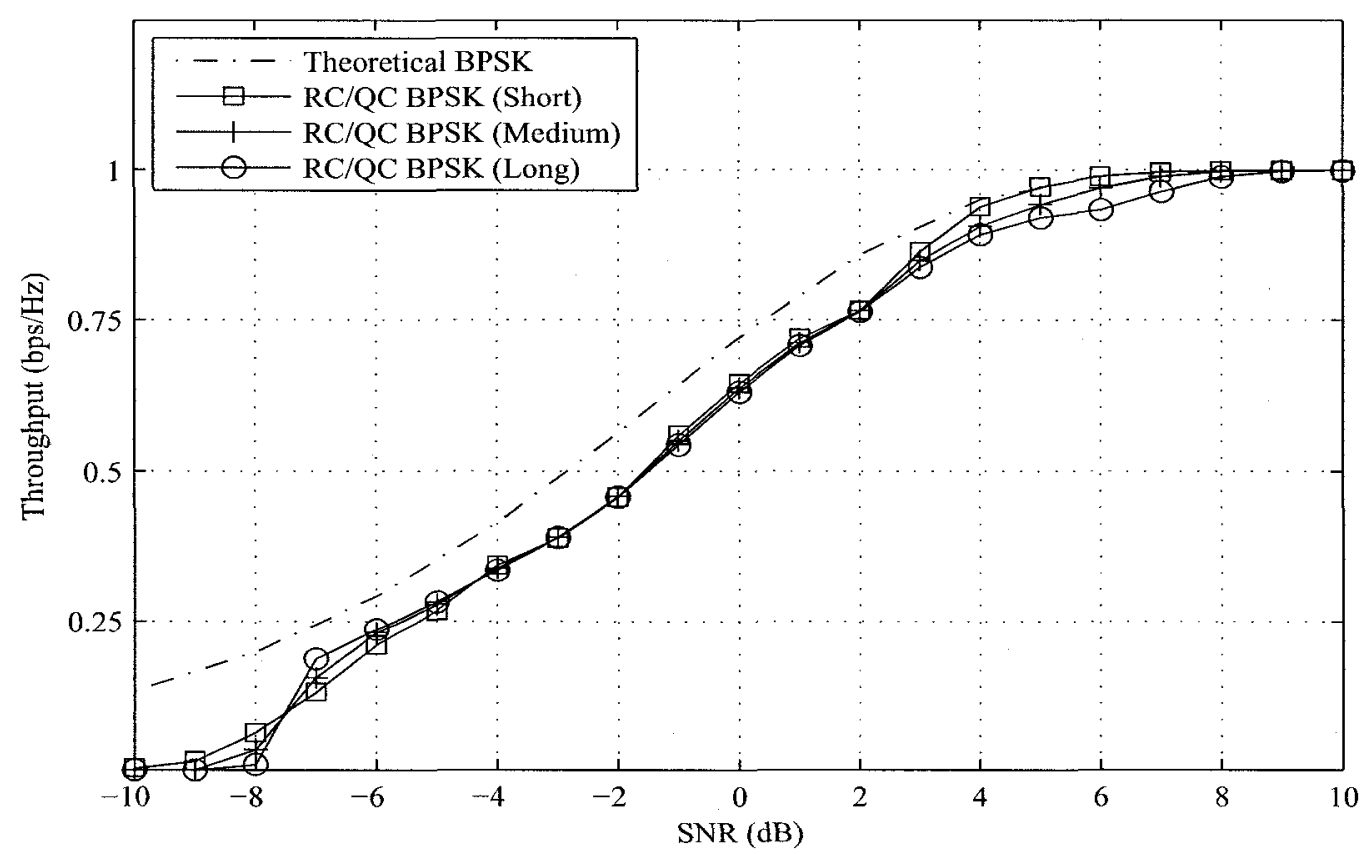

Figure 4.3: Throughput performance of RC/QC LDPC code using different codeword lengths and BPSK in an AWGN channel.

infallible nor rate-1, so in practice worse performance is expected, particularly for the shorter code. The exact degradation depends on the actual implementation of the CRC, and such analysis is beyond the scope of this thesis.

Figure 4.4 gives the performance of RC/QC LDPC codes when 4-QAM is used. The results are similar to those found in Figure 4.3, with a worst-case performance loss of about $1 \mathrm{~dB}$. We observe that the shortest code again outperforms longer ones. Figures 4.5 and 4.6 give the throughput performance of RC/QC LDPC codes using 16- and 64-QAM. These curves confirm the observations made from Figures 4.3 and 4.4, although the performance loss increases to about $2 \mathrm{~dB}$ with 16 -QAM.

Figures 4.3 - 4.6 demonstrate the adaptability of RC/QC codes to the instantaneous SNR. This shows that RC/QC codes are well suited for use in fading channels, 


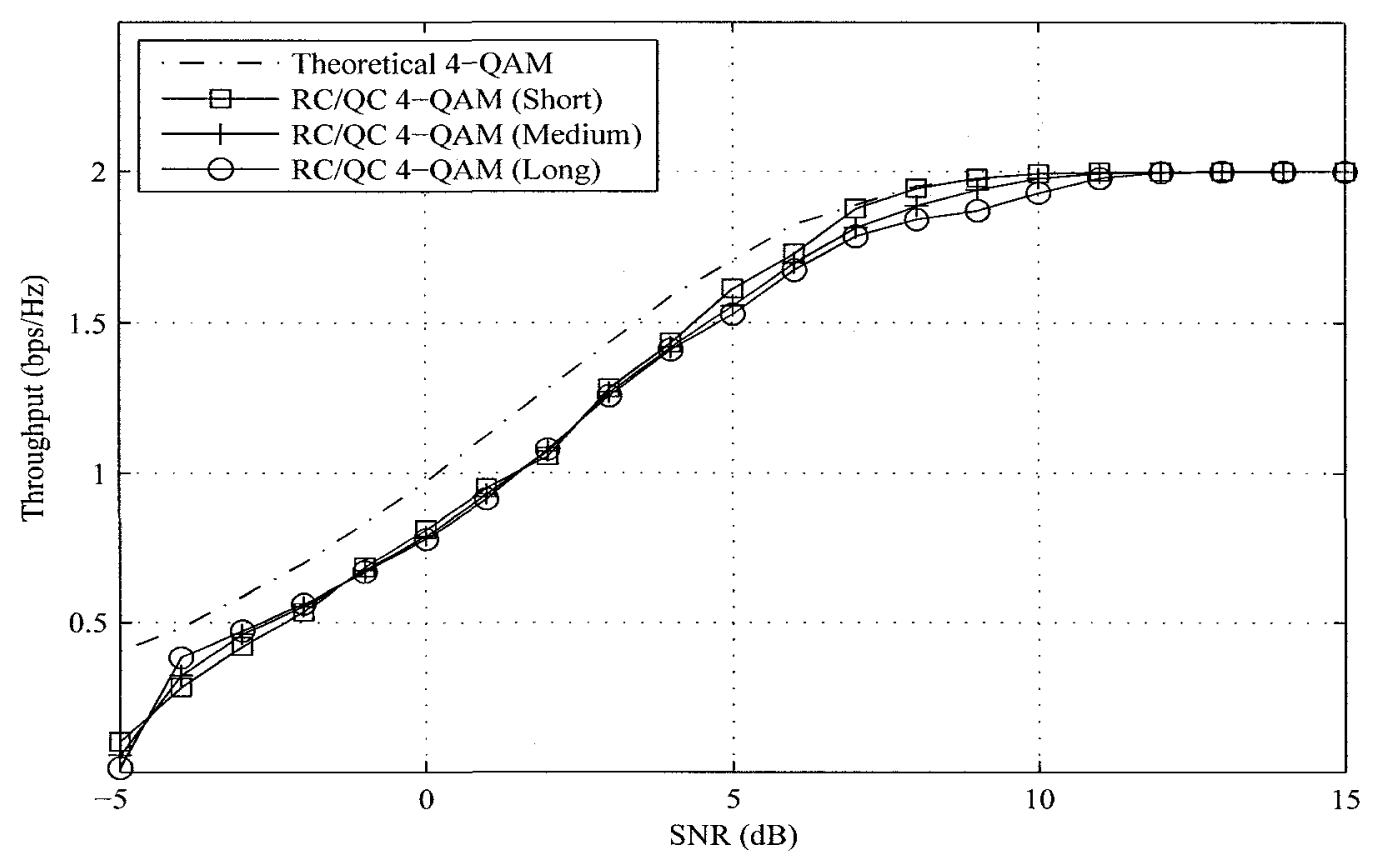

Figure 4.4: Throughput performance of RC/QC LDPC code using different codeword lengths and 4-QAM in an AWGN channel.

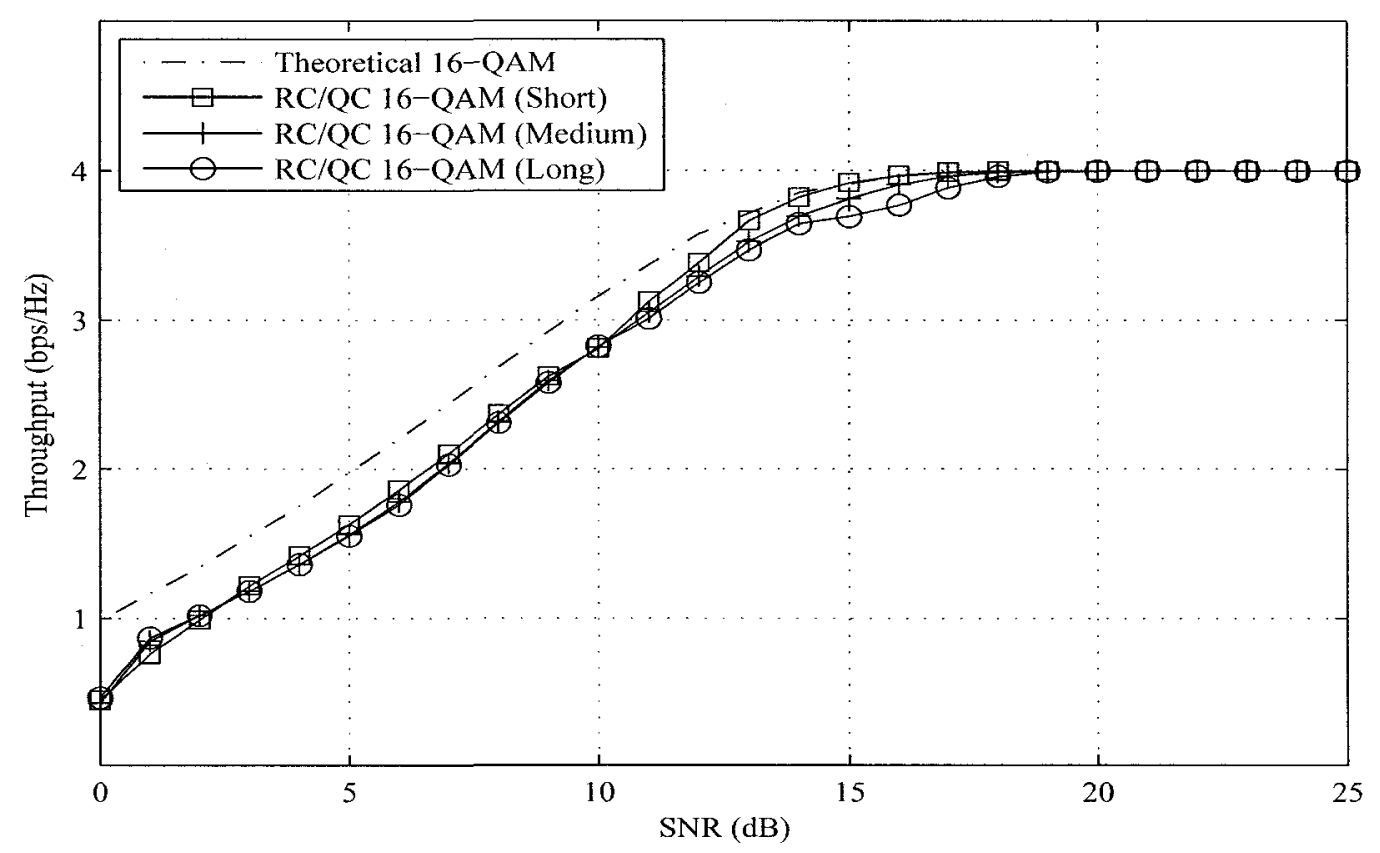

Figure 4.5: Throughput performance of RC/QC LDPC code using different codeword lengths and 16-QAM in an AWGN channel. 


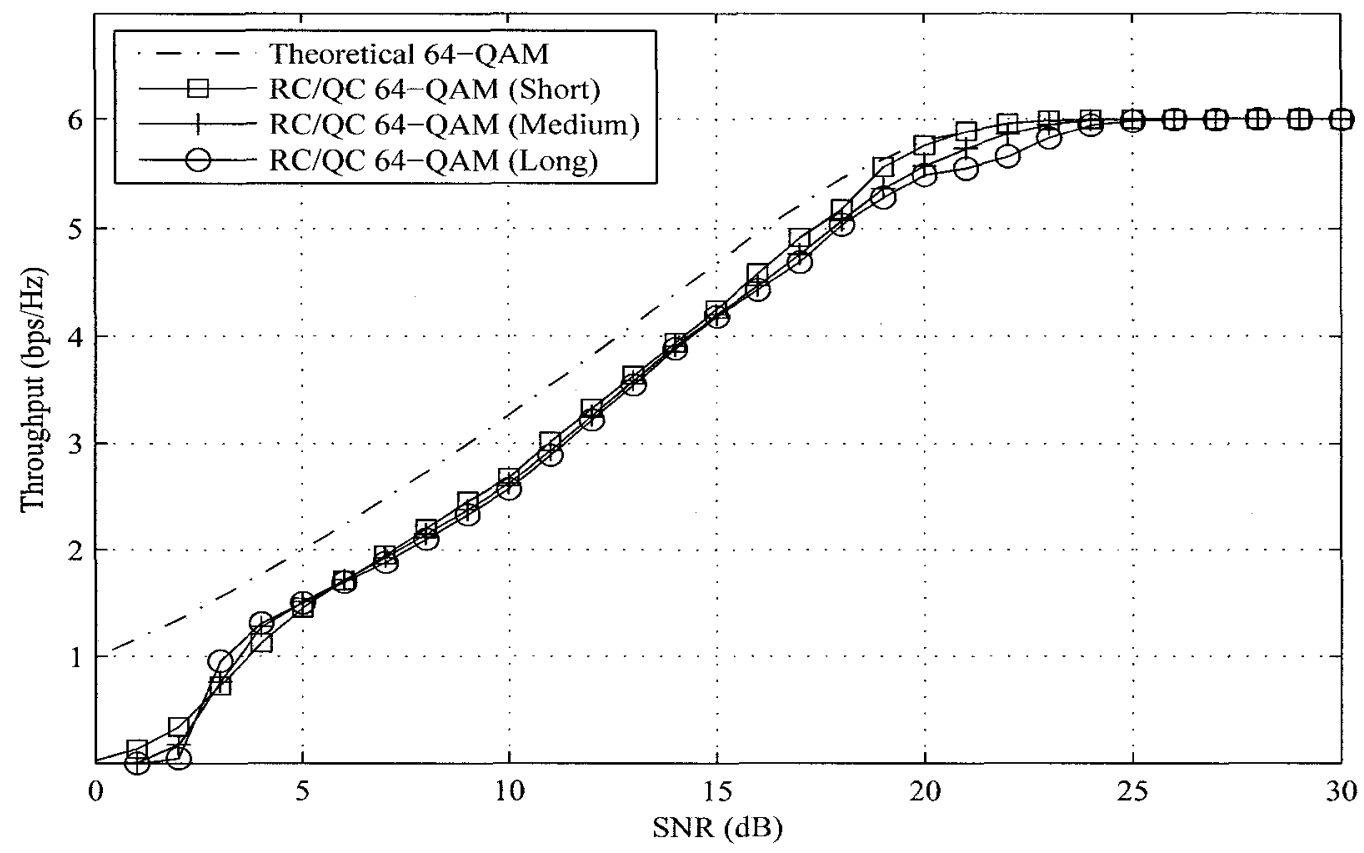

Figure 4.6: Throughput performance of RC/QC LDPC code using different codeword lengths and 64-QAM in an AWGN channel.

for which the instantaneous SNR fluctuates and depends on more than just the transmit and noise power. For convenience the 'short' length RC/QC LDPC codes are used in all future experimental simulations.

\subsubsection{Theoretical Constrained Throughput Performance of MIMO Transceivers}

In Chapter 3 we obtain curves for the unconstrained capacity of MIMO systems employing several different transceiver designs. In order to be able to evaluate the throughput performance of the MIMO systems described in this chapter we obtain curves for the constrained capacity of each MIMO transceiver. The constrained capacity curves are obtained using a look-up table made from (4.15) for the constrained 
capacity of a 64-QAM constellation. That is for a given channel realization we compute the instantaneous constrained capacity to be

$$
\mathrm{C}_{C N}=\sum_{i=1}^{L} \mathrm{C}_{64}\left(\phi_{i} \rho_{i}\right)
$$

where $\mathrm{C}_{C N}$ is the instantaneous constrained capacity of any given MIMO system; and $\mathrm{C}_{64}(\gamma)$ is the constrained capacity of 64 -QAM for an SNR $\gamma$. We run Monte Carlo simulations over 5000 channel realizations in order to obtain the average theoretical constrained capacity at each SNR. These results should be a foreshadowing to the results obtained for throughput performance of RC/QC-MIMO systems. They also allow us to gauge how much degradation in throughput performance is incurred by our implementation of the MIMO systems presented in Chapter 2.

For the purposes of fairness, all MIMO systems that require power allocation is done using the WFA. We also assume perfect MAI interference cancellation of already detected symbols. The rest of the interferers are considered Gaussian contributors to the SINR of each subchannel.

Figures 4.7 - 4.9 show the theoretical constrained throughput performance of IT transceivers for various numbers of antennas and a maximum constrained bit-rate of $6 \mathrm{bps} / \mathrm{Hz}$ per subchannel. From Figures $4.7-4.9$ we see that UCD has the best throughput performance followed very closely by ASGMD-MMSE. The theoretical throughput performance of the MMSE UT transceiver is included to give the reader an idea on the performance improvement that comes with using an IT when the rate is constrained. The MMSE transceiver is outperformed by all transceivers except the SVD-based ones. The SVD-WFA is no longer optimal due to the constrained rate of each subchannel as it wastes power on subchannels that have already reached the maximum rate. In fact, due to the waste of this energy the SVD-WFA has nearly the 


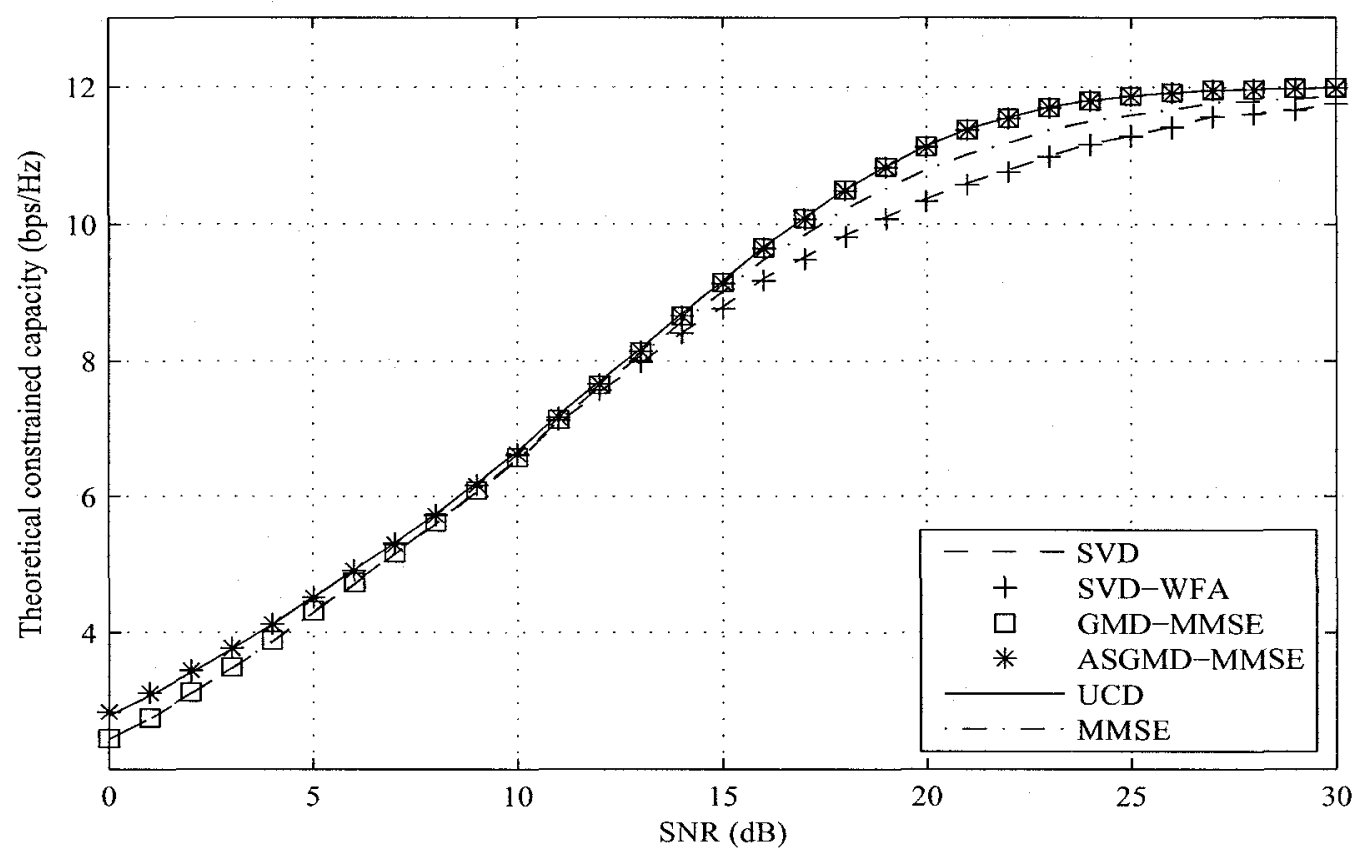

Figure 4.7: Theoretical throughput performance of rate-constrained $2 \times 2$ MIMO transceivers.

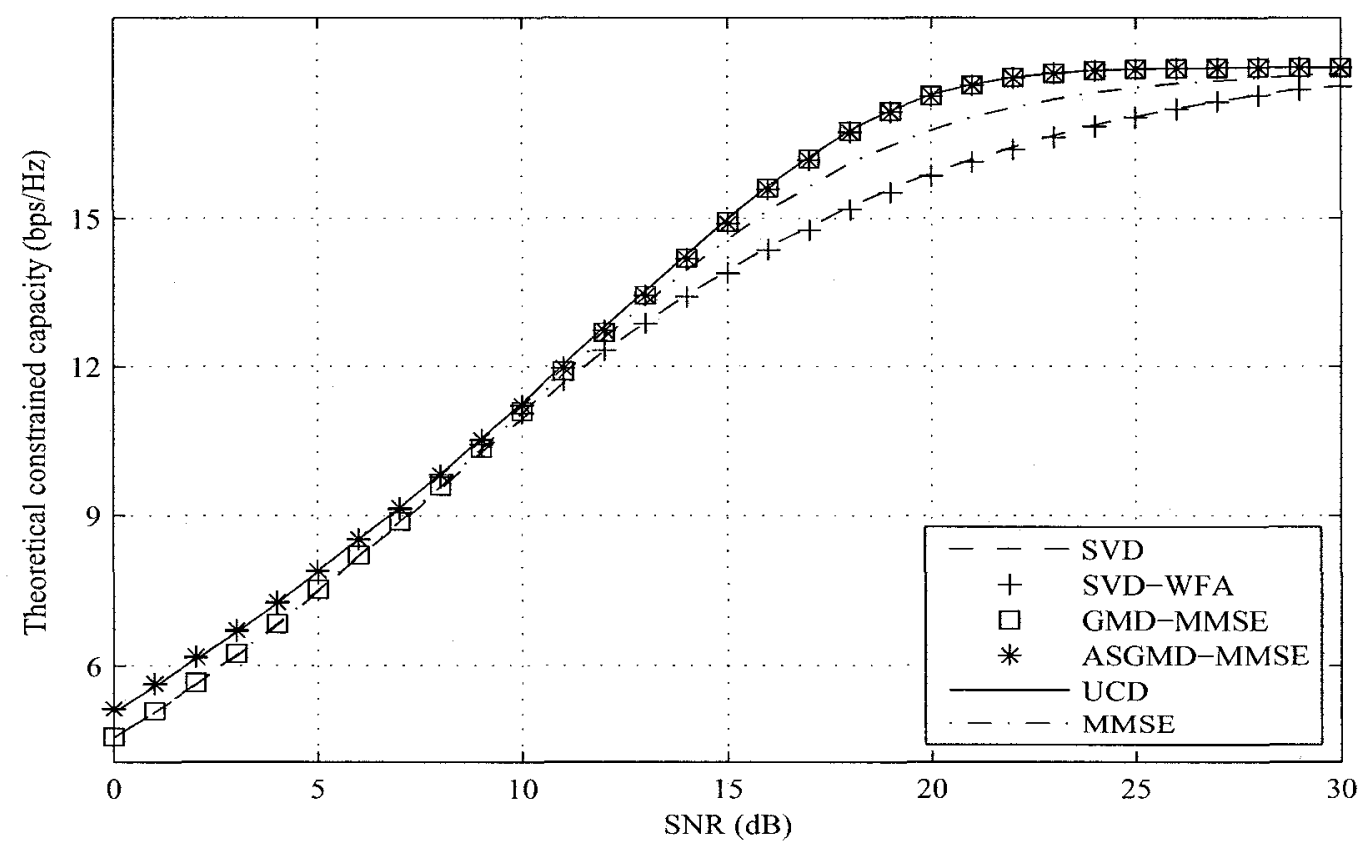

Figure 4.8: Theoretical throughput performance of rate-constrained $3 \times 3$ MIMO transceivers. 


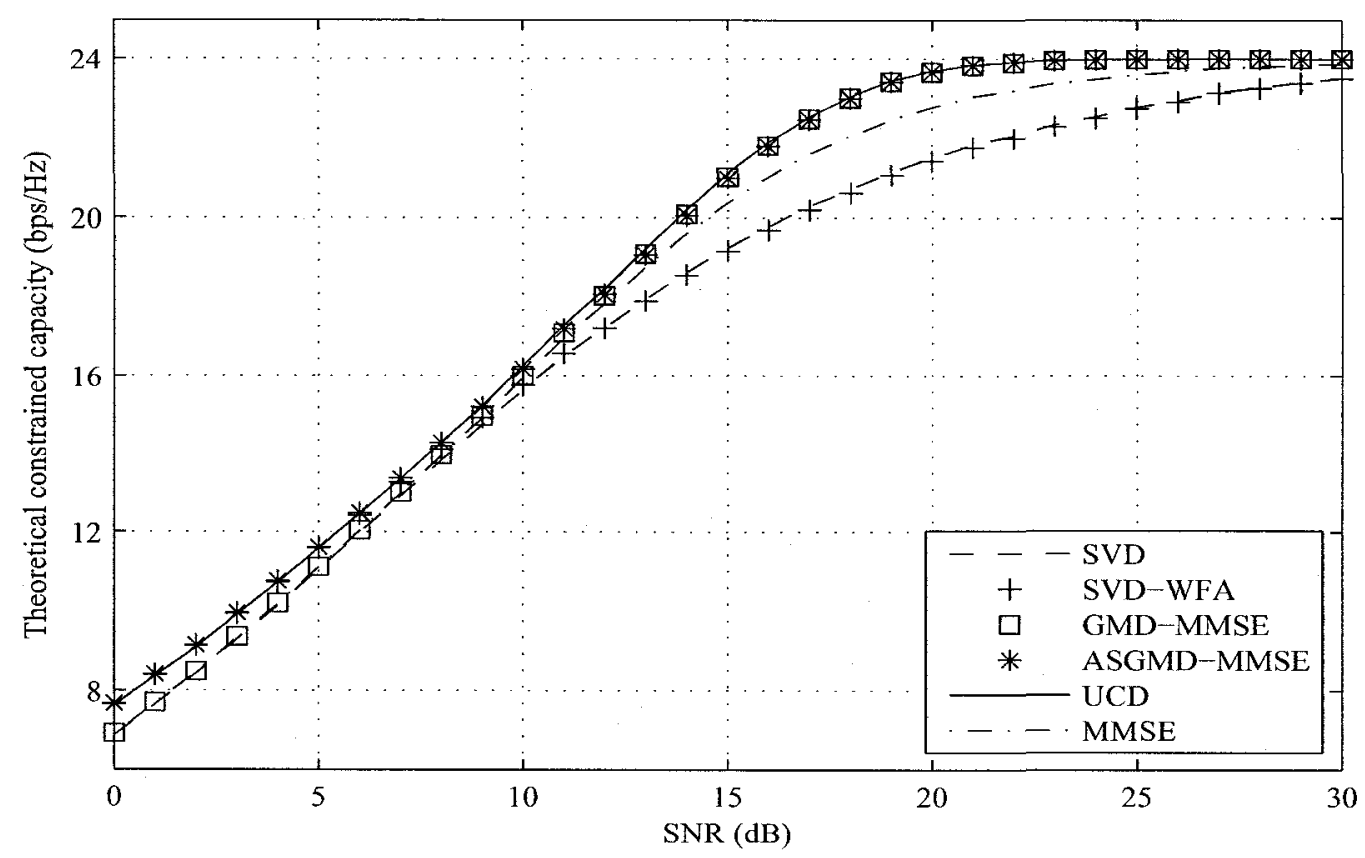

Figure 4.9: Theoretical throughput performance of rate-constrained $4 \times 4$ MIMO transceivers.

same performance as regular SVD. The SVD-based transceivers struggle to converge on the maximum rate due to the slow convergence of the poorest subchannel. The UCD transceiver has the same SINR on all subchannels and as a result suffers the least from this problem. That is to say, that all transceivers are throughput limited by the performance of their worst subchannel. A SVD decomposition has a large variance in its SINRs when compared with all other transceivers, and as a result has a tendency to have at least one poor subchannel. This is why a SVD transceiver saw no diversity for the BER curves shown in Chapter 3. On the other side of the coin, GMD- and UCD-based transceivers attempt to make all subchannels have the same gain and SINR respectively. This leads all subchannels to improve simultaneously, allowing these transceivers to keep their diversity as can be seen in Figures 4.7-4.9. Figure 4.10 demonstrates the aforementioned behavior. The plot is of the worst subchannels for each IT $4 \times 4$ MIMO transceivers. The worst subchannel of the GMD-based and 


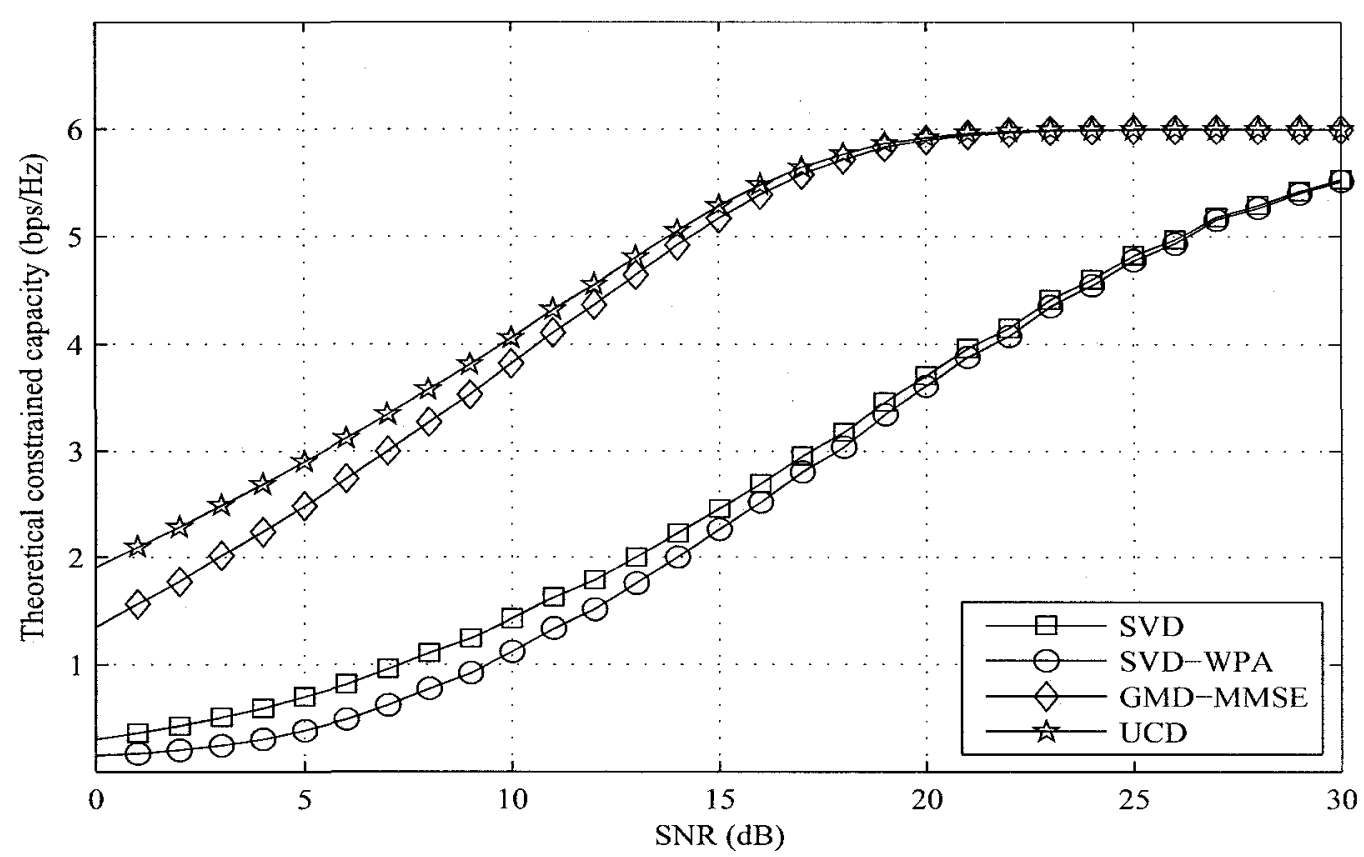

Figure 4.10: Theoretical throughput performance worst subchannel of $4 \times 4$ MIMO transceivers.

UCD far outperform those of the SVD-based transceivers - even with WFA. This shows how important equal gain subchannels are for rate-constrained MIMO systems. Yet, the results for MMSE, GMD- and UCD-based transceivers are optimistic. The assumption of no error propagation and perfect MAI cancellation is unrealistic as it is impossible to always be able to cancel MAI - otherwise we'd have a zero BER. However, these results do give a good idea of what can be achieved if a good detector is employed.

\subsubsection{Experimental Throughput Performance of RC/QC- MIMO}

In this subsection we evaluate the throughput performance of the RC/QC-MIMO system described earlier in this chapter using the ARR ${ }^{1}$. To re-iterate some of the

\footnotetext{
${ }^{1}$ For simplicity, in our simulations we did not consider the effects of packetization in decoding using incremental redundancy. That is, in simulation we computed all received samples for the
} 


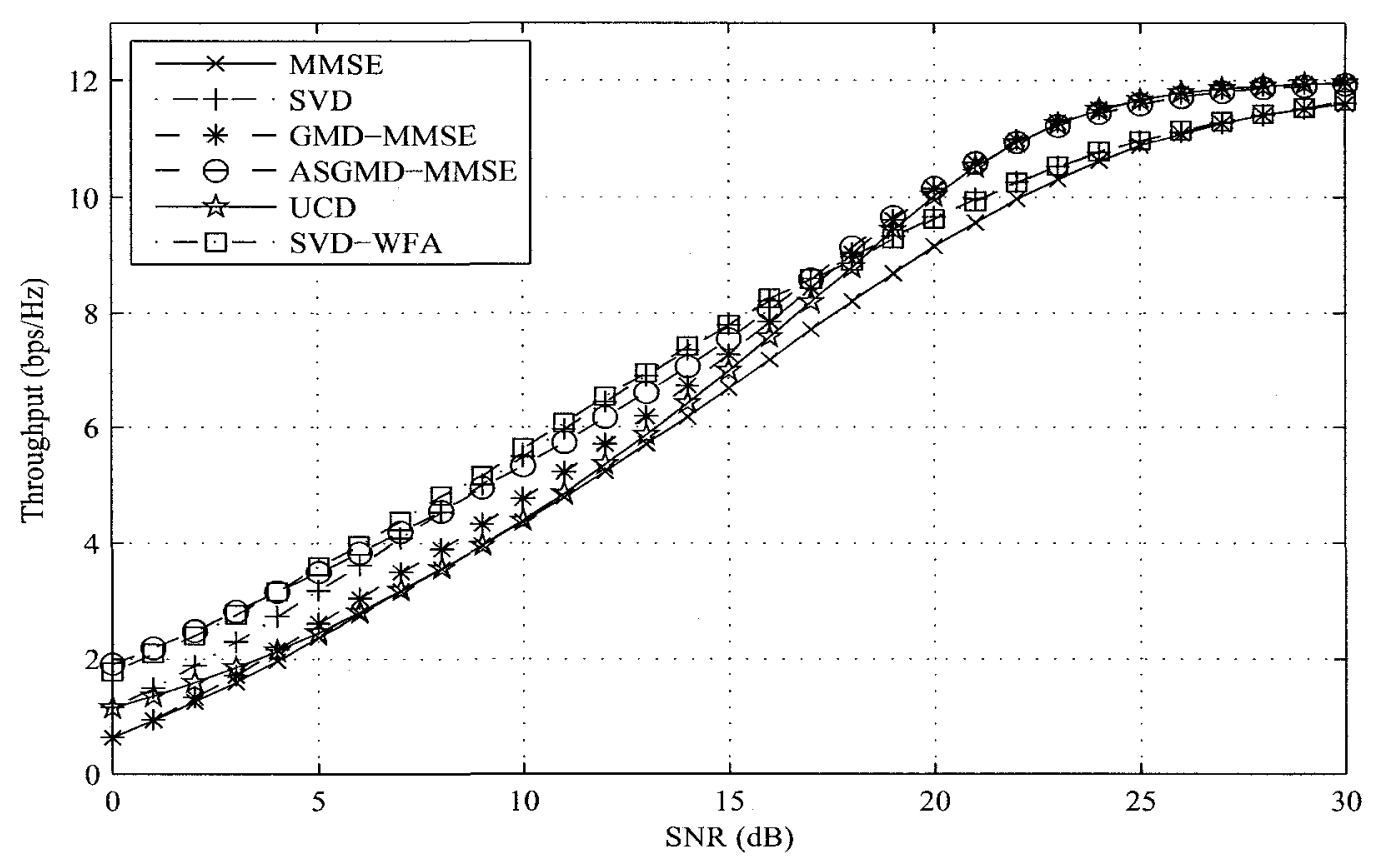

Figure 4.11: Experimental throughput performance of $2 \times 2$ RC/QC-MIMO with different transceiver designs.

operating parameters of the RC/QC-MIMO system: the symbol mappers are limited to using 64-QAM constellations; the detector operates under the assumption that all residual MAI has Gaussian statistics; and the WFA and antenna selection algorithms operate under the assumption that the capacity is unconstrained. All simulation results are collected for $L_{z}=3$.

Figure 4.11 gives the throughput performance of the $2 \times 2 \mathrm{RC} / \mathrm{QC}-\mathrm{MIMO}$ transceivers. What is most interesting, at first glance, is the difference in between the experimental and theoretical throughput performance curves. The ASGMD and UCD algorithms are not as dominant as they were in theory; and the MMSE maximum codeword length and did incremental redundancy by puncturing all non-systematic bits and then unpuncturing $L_{b}$ bits after each decoder failure. In reality, if the number of bits transmitted in one channel use is greater than $L_{b}$, puncturing should be done on a larger block size. We recognize that more accurate results could be obtained if we punctured the blocks based on the actual number of bits transmitted in one interval. 
transceiver performs far worse than the SVD-based transceivers, which is not the case in Figure 4.7. The MAI cancellation abilities of each transceiver has a large impact on the throughput performance of the MMSE, GMD-based and UCD transceivers. Recalling the BER performance curves given in Chapter 3 for the IT transceivers, we saw that the GMD-MMSE transceiver had better performance than the UCD transceiver for larger constellation sizes; in one case outperforming it for SNRs of up to $29 \mathrm{~dB}$. Thus, RC/QC-UCD is outperformed by RC/QC-GMD and RC/QC-ASGMD. In addition, the MMSE theoretical constrained capacity curves were overly optimistic - and they should be due to the perfect MAI cancellation assumption. The RC/QC-MMSE transceiver performs the worst of all transceivers because it has no diversity and, as a result, has more difficulty in resolving the MAI in its decision variables. The SVD-based transceivers do not experience MAI and as a result do not lose performance as a result of it. RC/QC-ASGMD is the best non-SVD based MIMO system. At low SNRs it outperforms both SVD-based transceivers because it most often uses one subchannel and as a result experiences no MAI. As the SNRs become larger it begins to choose larger sets of subchannels. This leads to a greater chance of MAI irresolvability, and as a result, the RC/QC-ASGMD curve begins to "dip" for mid-range SNRs, in the range where it starts using more subchannels.

An interesting result is that RC/QC-GMD converges on the system bit-rate maximum faster than RC/QC-ASGMD. This happens because the antenna selection algorithm of ASGMD is based upon the unconstrained capacity. At higher SNRs RC/QC-GMD and RC/QC-ASGMD are able to better resolve the MAI in its decision variables and again begin to outperform RC/QC-SVD and RC/QC-SVD-WFA due to its ability to converge on the maximum nominal bit-rate. RC/QC-SVD-WFA confirms the conjecture that the WFA has poor performance in a constrained communication system. At higher SNRs it is even outperformed by RC/QC-SVD. 


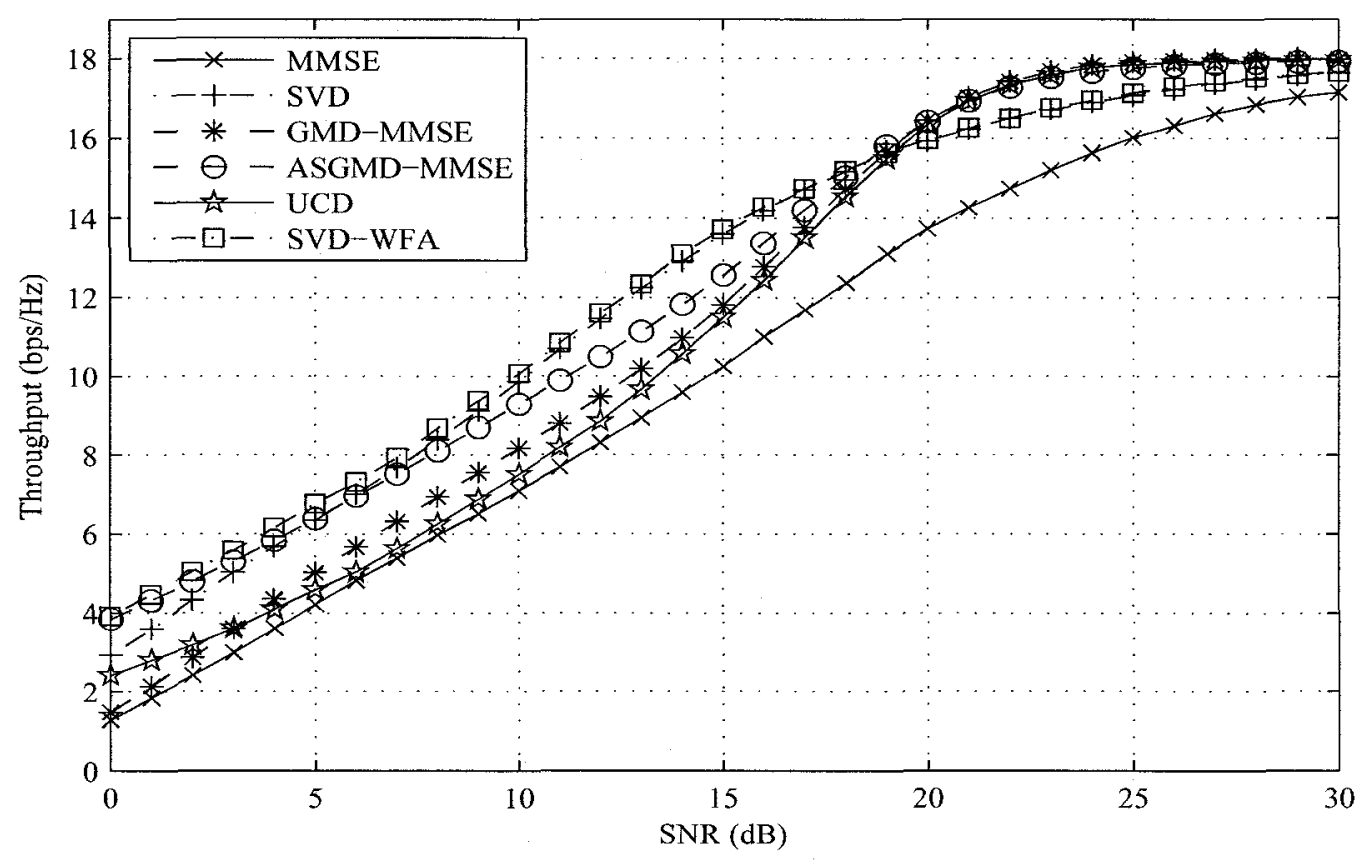

Figure 4.12: Experimental throughput performance of $3 \times 3 \mathrm{RC} / \mathrm{QC}-\mathrm{MIMO}$ with different transceiver designs.

Figures 4.12 and 4.13 give the performance of the $3 \times 3$ and $4 \times 4$ RC/QC-MIMO systems. RC/QC-SVD and RC/QC-SVD-WFA dominate at mid-range SNRs due to the fact that they do not have any MAI in their decision variables. For Figures 4.12 and 4.13 the effect of MAI is even more pronounced, as can be seen by the growth in the size of the "dip" that exists for MIMO transceivers with MAI at mid-range SNRs. RC/QC-MMSE struggles by comparison to the IT RC/QC-MIMO systems. For an SNR of $20 \mathrm{~dB}$ there is a spectral efficiency gain of roughly $2.1 \mathrm{bps} / \mathrm{Hz}$ and 2.8 bps $/ \mathrm{Hz}$ for the second worst performing RC/QC-MIMO transceiver over the $3 \times 3$ and $4 \times 4 \mathrm{RC} / \mathrm{QC}-\mathrm{MMSE}$ systems respectively. This result shows that the impact of MAI irresolvability grows with the antenna array size. It also clearly demonstrates the advantage of using an IT over an UT transceiver for bit-rate constrained MIMO. The UCD transceiver, even with its extra complexity does not perform well. This 


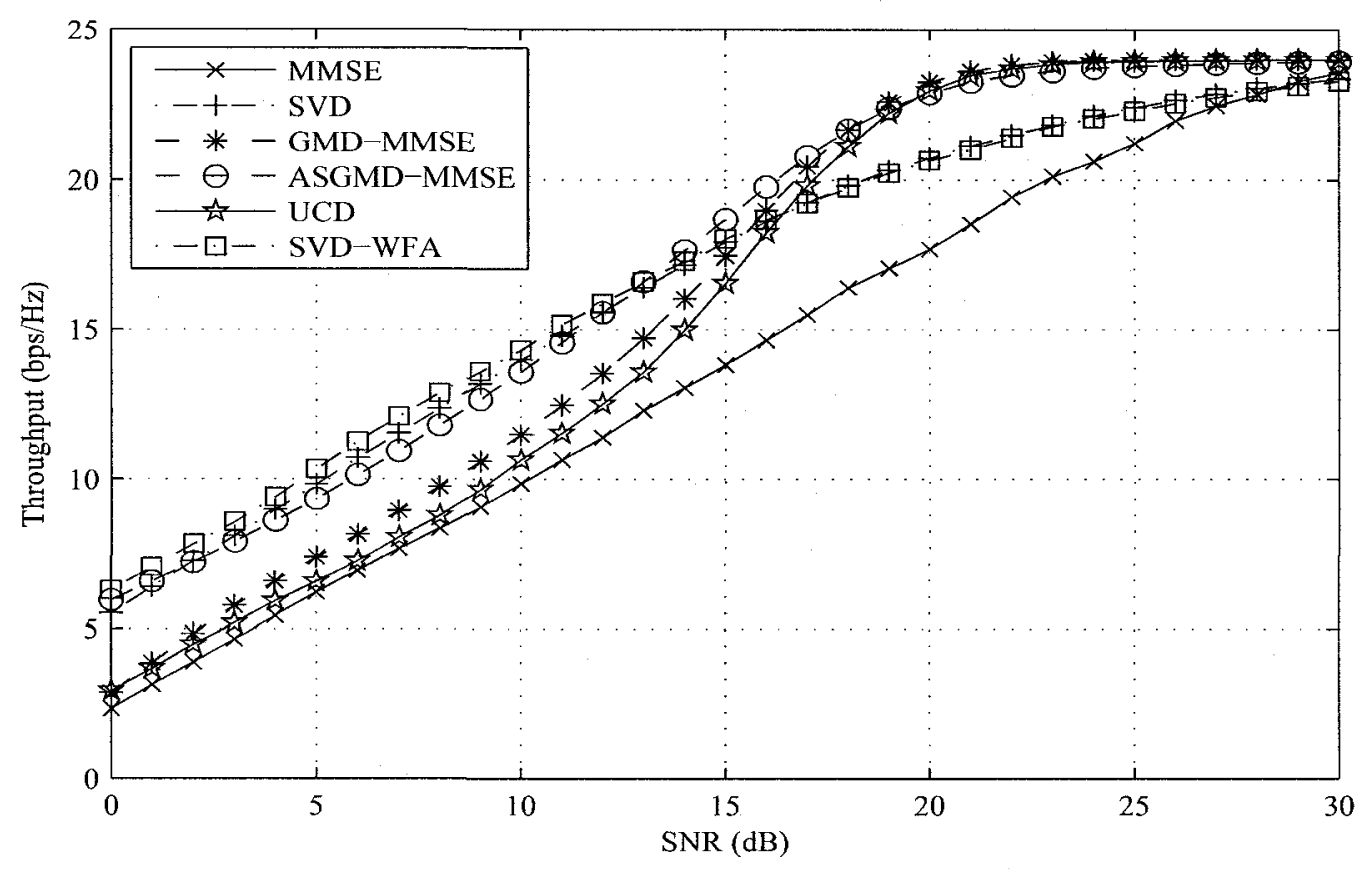

Figure 4.13: Experimental throughput performance of $4 \times 4 \mathrm{RC} / \mathrm{QC}-\mathrm{MIMO}$ with different transceiver designs.

was foreshadowed by its BER curves in Chapter 3 which demonstrated that it is outperformed by both the GMD- and SVD-based transceivers for low to mid-range SNRs, and the GMD-based transceiver for SNRs of up to $29 \mathrm{~dB}$. This shows that although a MIMO transceiver may be well designed for unconstrained capacity and even provide high diversity, it may not perform well when the bit-rate is constrained. The struggles of RC/QC-ASGMD to converge with the bit-rate maximum is even more evident for larger antennas arrays. In Figures 4.11 and 4.12 the SNR for which RC/QC-GMD begins to outperform RC/QC-ASGMD is about $17.9 \mathrm{~dB}$ and $20.7 \mathrm{~dB}$ for the $3 \times 3$ and $4 \times 4$ MIMO systems respectively; as opposed to $23.4 \mathrm{~dB}$ for the $2 \times 2$ MIMO systems. 


\section{Chapter 5}

\section{Power and Bit-Rate Allocation for the Rate-Constrained MIMO Subchannel}

In Chapter 4 we implemented RC/QC-MIMO systems that did not use adaptive bitrate allocation. That is, the transmitter could only send 64-QAM constellations on $L$ subchannels. While RC/QC LDPC codes are capable of adapting the data rate without regard to the type of modulation used, it has a lower rate limit of

$$
\eta_{\min }=6 L R_{c}^{(\min )}
$$

where $\eta_{\min }$ is the nominal lowest rate experienced a RC/QC MIMO system. For our implementation of RC/QC LDPC codes described in Appendix B, $R_{c}^{(m i n)}=\frac{2}{11}$. This makes lowest possible nominal rate $\eta_{\min }=\frac{12}{11}$ for the case when only one subchannel is used. This means that the channel capacity only needs to be below $1 \mathrm{bps} / \mathrm{Hz}$ to make it unlikely that our detector will be able to decode the codeword. Furthermore, for multiple subchannel transmissions, the bit-rate that each subchannel is capable of supporting may be different. However, a 64-QAM constellation is assigned to all $L$ subchannels, regardless of their constrained capacity. For a SCW implementationbased RC/QC-MIMO system, where the SINR of all subchannels has an impact on the entire codeword, it is better to assign a bit-rate to each subchannel that reflects 
its instantaneous capacity.

The power-allocation algorithm of the RC/QC-MIMO system of Chapter 4 assigned powers to each subchannel using the WFA, uses the unconstrained capacity of a MIMO channel. However, the bit-rate on each subchannel is constrained. This means that if the power assigned to a subchannel by the WFA is such that the bit-rate that the WFA could assign is in excess of the maximum bit-rate, unnecessary energy is being allocated to that subchannel. By wasting energy the RC/QC-MIMO system will not able to achieve its potential throughput performance.

The RC/QC-MIMO system of Chapter 4 does not take advantage of adaptive modulation and fails to allocate energy to each subchannel using full knowledge of its operation limitations. In this chapter we propose two new WFAs that use adaptive modulation based upon a discrete set of constellations and do power allocation based on the constrained rate of each subchannel.

\subsection{CWFA: Power and Bit-Rate Allocation Algo- rithms}

The symbol mapper of Figure 4.1 is allowed to use adaptive modulation using BPSK, 4-QAM, 16-QAM and 64-QAM. As in Chapter 4 the maximum constellation size is 64 . This means that the set of choices for the constellation size in doing bit-rate allocation on each subchannel is $\mathcal{B}=\{2,4,16,64\}$. Given a system with IT or even PIT the transmitter is able to assign a constellation of size $M_{i} \in \mathcal{B}$, to the $i^{\text {th }}$ subchannel based upon feedback relayed from the receiver, depending upon the SINRs of each subchannel. 


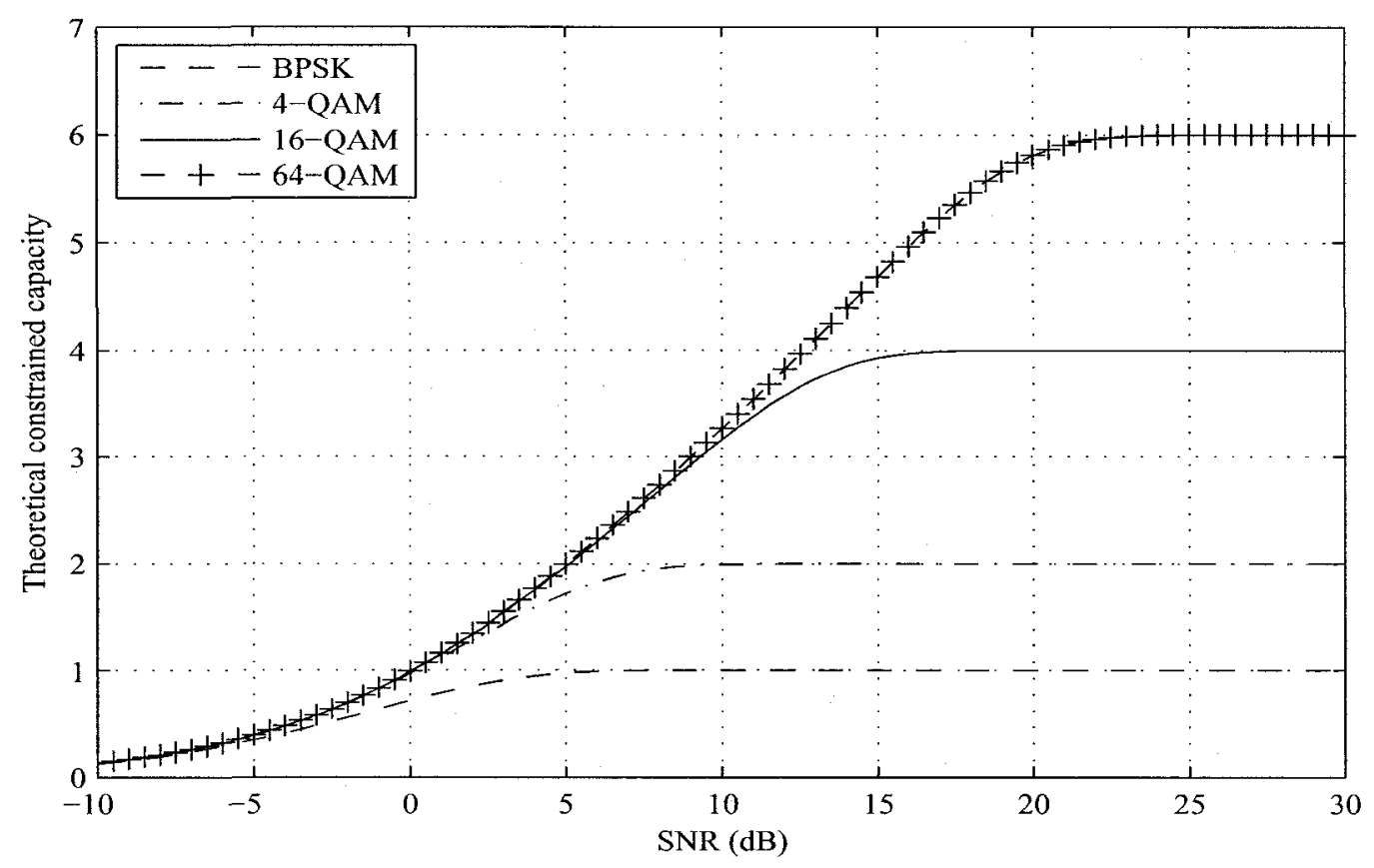

Figure 5.1: Theoretical constrained capacity of $M$-QAM in an AWGN channel.

For the case of a MIMO system with unconstrained rate, powers and bit-rates are assigned using the WFA. However, in the case where rate is constrained the WFA is no longer the best method for power- and bit-loading. For the constrained MIMO system, a new CWFA is designed that attempts to maximize the throughput of the MIMO system. Given that the MIMO system uses QAM constellations with sizes from the set $\mathcal{B}$, the CFWA should do its optimization based upon the actual constrained capacities of these QAM constellations. Theoretically, these capacities are computed using (4.15) and shown in Figure 5.1. It can be seen that all $M$-QAM capacity curves overlap each other until they approach their respective maximum bitrates. This means that the maximum size constollation (64-QAM) will theoretically always give better constrained capacity performance than all other constellations in $\mathcal{B}$. Therefore, we only need consider the 64-QAM capacity curve when doing power allocation. However, since we use RC/QC LDPC codes the bit-rate that each 


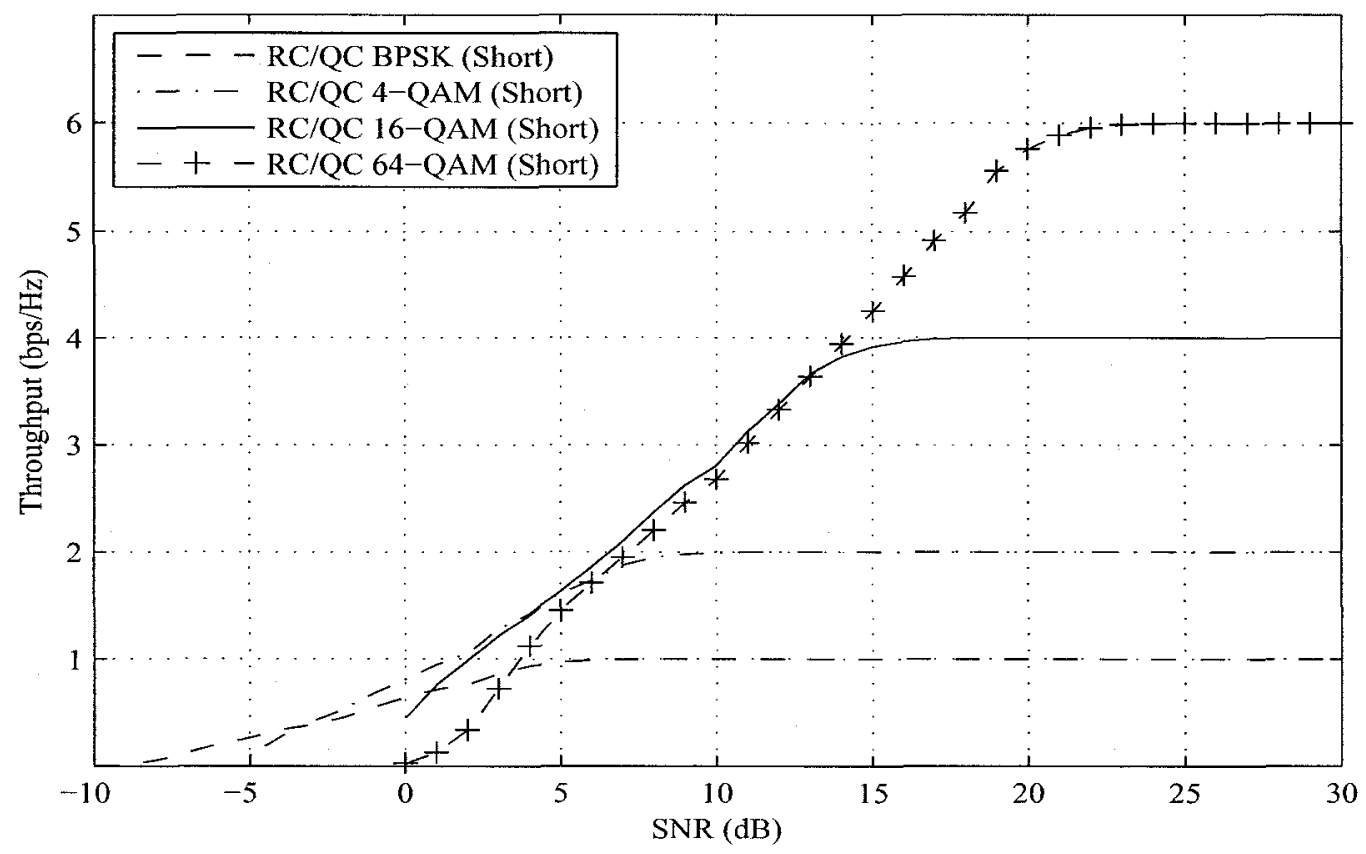

Figure 5.2: Experimental throughput of 'short' RC/QC LDPC codes with different $M$-QAM constellations in an AWGN channel.

subchannel can support is different from the result given by (4.15). Figure 5.2 gives the RC/QC LDPC code performance for different constellation sizes in an AWGN channel. Figure 5.2 not only demonstrates that there is a loss in throughput for $\mathrm{RC} / \mathrm{QC}$ LDPC codes when compared to the theoretical constrained capacity, but that the throughput performance of 64-QAM does not dominate at all SNRs. Thus, it is better to do power and bit-rate allocation for a RC/QC-MIMO system using the results given in Figure 5.2.

\section{Numerical CWFA (NCWFA)}

A power allocation matrix $\Phi$ can be determined numerically by maximizing the sum of the constrained capacities of all of the subchannels. We call this algorithm numerical CWFA (NCWFA). For the theoretical MIMO system, the algorithm attempts to find

$$
\boldsymbol{\Phi}=\arg \max _{\mathbf{\Phi}} \sum_{j=1}^{L} \mathrm{C}_{64}\left(\phi_{j} \rho_{j}\right)
$$


where $\phi_{j}$ is the fraction of power assigned to subchannel $j$ with SINR $\rho_{j}$. The numerical maximization algorithm is defined by a $N_{t}$-dimensional search space, which can be reduced to a $N_{t}-1$ search space using the energy constraint defined in Section 2.1. For example, if $N_{t}=2$ the powers $\phi_{1}$ and $\phi_{2}$ are related by the following

$$
\phi_{2}=\frac{1-\alpha}{\alpha} \phi_{1}
$$

where $\phi_{1}=\alpha E_{s} N_{t}$. The search can then be done using only $\alpha$, reducing the search space from two to one dimension. The NCWFA is feasible for a small number of transmit antenna $\left(N_{t} \leq 3\right)$, however for larger numbers of transmitting antennas it quickly becomes computationally exhaustive. Here we have designed the NCWFA for the theoretical-constrained-capacity MIMO system. It could also be designed using the experimental throughput curves of Figure 5.2.

\section{Simple CWFA (SCWFA)}

To achieve decent throughput, while keeping the computational cost of a CWFA low, we propose a modification on the existing WFA. This recursive power and bit-rate allocation algorithm assigns powers using a capped version of the unconstrained capacity and chooses an appropriate rate based upon the experimental throughput curves of Figure 5.2. The modified version of the WFA - called simple CWFA (SCWFA) attcmpts to resolve the power allocation problem that the WFA suffers from when implemented in constrained rate MIMO systems. Instead of unnecessarily wasting additional power on subchannels that can already achieve the maximum bit-rate, SCWFA re-assigns that power to other subchannels that do not have enough power to reach the maximum rate. The SCWFA can be viewed as a recursive WFA, where if some of the power allocations assigned using the WFA are wasteful, a power is assigned to the subchannel with the largest SINR that allows it to reach the maximum 
rate and the number of subchannels with assigned powers, $a$, is increased by one. The power allocation portion of the WFA is then recomputed using the remaining subchannels with unassigned powers, $b$, and the remaining unallocated power, $P_{r}$. The algorithm runs until every subchannel has a power allocated to it and then does bit-rate allocation based on the power allocated to and SINR of each subchannel using a look-up table based on Figure 5.2. The algorithm is as follows:

1. Order the SINRs, $\left\{\rho_{i}\right\}$, in descending order for $i \in\{1, \ldots, K\}$.

2. Set $a=0, b=K, P_{r}=N_{t}$.

3. Calculate $\mu=\frac{1}{b}\left(P_{r}+\sum_{i=1}^{b} \frac{1}{\rho_{i}}\right)$ and $\phi_{i}=\mu-\frac{1}{\rho_{i}} \forall i \in\{a, \ldots, K\}$

4. If $\phi_{b} \leq 0$ or $\mathrm{C}_{64}\left(\phi_{b} \rho_{b}\right)<R_{c}^{(\min )} ; b=b-1$ and go to 3$)$. Else go to 5$)$.

5. If $\phi_{a}>\left(2^{6}-1\right) \frac{1}{\rho_{a}}$; set $\phi_{a}=\left(2^{6}-1\right) \frac{1}{\rho_{a}}, b=K, P_{r}=P_{r}-\phi_{a}, a=a+1$ and go to 3$)$. Else go to 6$)$.

6. $P_{r}=P_{r}-\sum_{i=a}^{K} \phi_{i}$

7. If $P_{r}>0$ redistribute remaining power: $\phi_{i}=\phi_{i}+\frac{P_{r}}{b} \forall i \in\{1, \ldots, b\}$

8. Set $L=b$.

9. Choose a constellation size for each subchannel using $M_{i}=\mathrm{R}_{T}\left(\left(\frac{\phi_{i}}{N_{i}}\right)_{d B}\right)$.

where $R_{T}\left(\gamma_{d B}\right)$ is a rate-list look-up table that assigns a constellation in $\mathcal{B}$ based upon a SNR, $\gamma_{d B}$, in $\mathrm{dB}$. The rate-list look-up table uses the experimental throughput performance of a 'short' $\mathrm{RC} / \mathrm{QC} \mathrm{LDPC}$ for the constellations of $\mathcal{B}$ in an AWGN channel. The rate-list look-up table chooses an appropriate value from $\mathcal{B}$ using the 
following:

$$
R_{T}\left(\gamma_{d B}\right)= \begin{cases}0 & \text { if } \gamma_{d B}<-6.125 \mathrm{~dB} \\ 2 & \text { if }-6.125 \mathrm{~dB} \leq \gamma_{d B} \leq-3.323 \mathrm{~dB} \\ 4 & \text { if }-3.323 \mathrm{~dB} \leq \gamma_{d B} \leq 4.53 \mathrm{~dB} \\ 16 & \text { if } 4.53 \mathrm{~dB} \leq \gamma_{d B} \leq 13.19 \mathrm{~dB} \\ 64 & \text { otherwise }\end{cases}
$$

Note that $-6.125 \mathrm{~dB}$ is approximately the SNR where the rate that can be supported on the AWGN channel is equal to $R_{c}^{(m i n)}$. This is a suboptimal CWFA because it does not use the appropriate capacity curve to do power allocation. The SCWFA, being based upon a capped version of the unconstrained capacity curve, overestimates the throughput performance of the subchannel and removing some power from the best subchannels can actually reduce their throughput below the maximum as a result. A modification can be made such that step five is changed to higher a power assignment to $\phi_{a}=\gamma_{\max } \frac{1}{\rho_{a}}$; where $\gamma_{\max }$ is the SNR for which the AWGN throughput of the 64-QAM RC/QC LDPC code is roughly six. Doing this ensures that the best conditioned channels are always able to achieve the maximum throughput, should they be capable. This adaptation is used for our implementation of SCWFA in this thesis.

The bit loading of the SCWFA is based upon the AWGN experimental throughput performances of the QAM constellations and, as a result, the assigned bit-rate does reflect the capacity of the subchannel for the given SINR and power assignment. We note that because the UCD transceiver does power allocation based on the WFA, it cannot use the power allocation portion of the CWFAs. This exception does not apply to any of the other MIMO transceivers described in Section 2.3 of Chapter 2. 


\subsection{Rate-Constrained Antenna Selection Algo- rithm for ASGMD}

The ASGMD transceiver of Chapter 2 selects the appropriate number of subchannels to transmit on, so as to increase the unconstrained capacity of a GMD-based transceiver. As we have already mentioned earlier in this chapter, the RC/QC-MIMO system has constrained rate and as a result the antenna selection algorithm of the ASGMD transceiver is not appropriate. Thus, we adapt to the antenna selection algorithm of the ASGMD transceiver to reflect the rate-constraint of the RC/QC-MIMO system. The ASGMD is made to select the appropriate number of antennas using

$$
L=\max _{L \in[1, K]} \sum_{j=1}^{L} \mathrm{~T}_{\tau_{j}}\left(\frac{N_{t}}{L} \rho_{j}\right)
$$

where $\tau_{j}=\mathrm{R}_{T}\left(\left(\frac{N_{t}}{L} \rho_{j}\right)_{d B}\right)$; and $\mathrm{T}_{\tau_{j}}$ is a look-up table giving the rate obtained using a $\tau_{j}$-QAM constellation at a given SNR. These look-up tables are created from the throughput performance of the different QAM constellation given by Figure 5.2. We call this algorithm the constrained capacity antenna selection algorithm (CCAS).

\subsection{Performance Evaluation of CWFAs}

In this section we evaluate the theoretical MIMO and experimental RC/QC-MIMO throughput performance when transceivers use the CWFAs.

\subsubsection{Theoretical Throughput Performance of CWFAs}

Two new CWFAs were introduced in Section 5.1. The theoretical capacity of a MIMO transceiver is based upon the SINRs of and powers allocated to each subchannel. This means that the theoretical throughput will not be able to give 
any idea of the efficacy of the bit-rate allocation portion of the CWFA. Most of the transceivers (SVD,GMD-MMSE,ASGMD-MMSE,UCD) introduced in Chapter 2 do not make use of a CWFA to assign their powers, since their powers are either fixed for the number of selected subchannels or their powers are allocated using a different algorithm. This means that the only transceiver for which we can evaluate the theoretical efficacy of the power allocation portion of the CWFAs is SVD-WFA. Note that in this evaluation, the power threshold of SCWFA, $\gamma_{\max }$, is chosen such that $\mathrm{C}_{64}\left(\gamma_{\max }\right) \approx 6$. This is done to reflect the fact that we are evaluating the SCWFA for a theoretical MIMO system. In addition, CCAS is adapted to use $\mathrm{C}_{64}(\cdot)$ to do antenna selection for the theoretical rate-constrained MIMO system.

Figure 5.3 demonstrates the performance improvement seen when using a CWFA versus a regular WFA for a $2 \times 2$ MIMO system. The best of the CWFAs is the NCWFA as should be expected since it based on numerical optimization. It is encouraging to see that the SCWFA does not lose too much in throughput performance compared to the NCWFA. The use of the capped unconstrained capacity curve explains the small loss in performance, since this curve gives overly optimistic predictions about the throughput of each subchannel. As a result the SCWFA has a tendency to overestimate the performance of a subchannel for higher SNRs and make power assignments that are not as precise as the NCWFA. At a SNR of $20 \mathrm{~dB}$ the SWCFA has only a $0.1 \mathrm{bps} / \mathrm{Hz}$ loss in spectral efficiency, compared to a $0.3 \mathrm{bps} / \mathrm{Hz}$ loss for the regular WFA algorithm. The WFA struggles to achieve the same theoretical constrained throughput because it is allocating power on subchannels that have already achieved the maximum rate, while the CWFAs use this extra power to improve the rates of other subchannels. The NCWFA becomes computationally exhaustive for larger numbers of antennas and is closely matched in throughput performance by the less computationally 


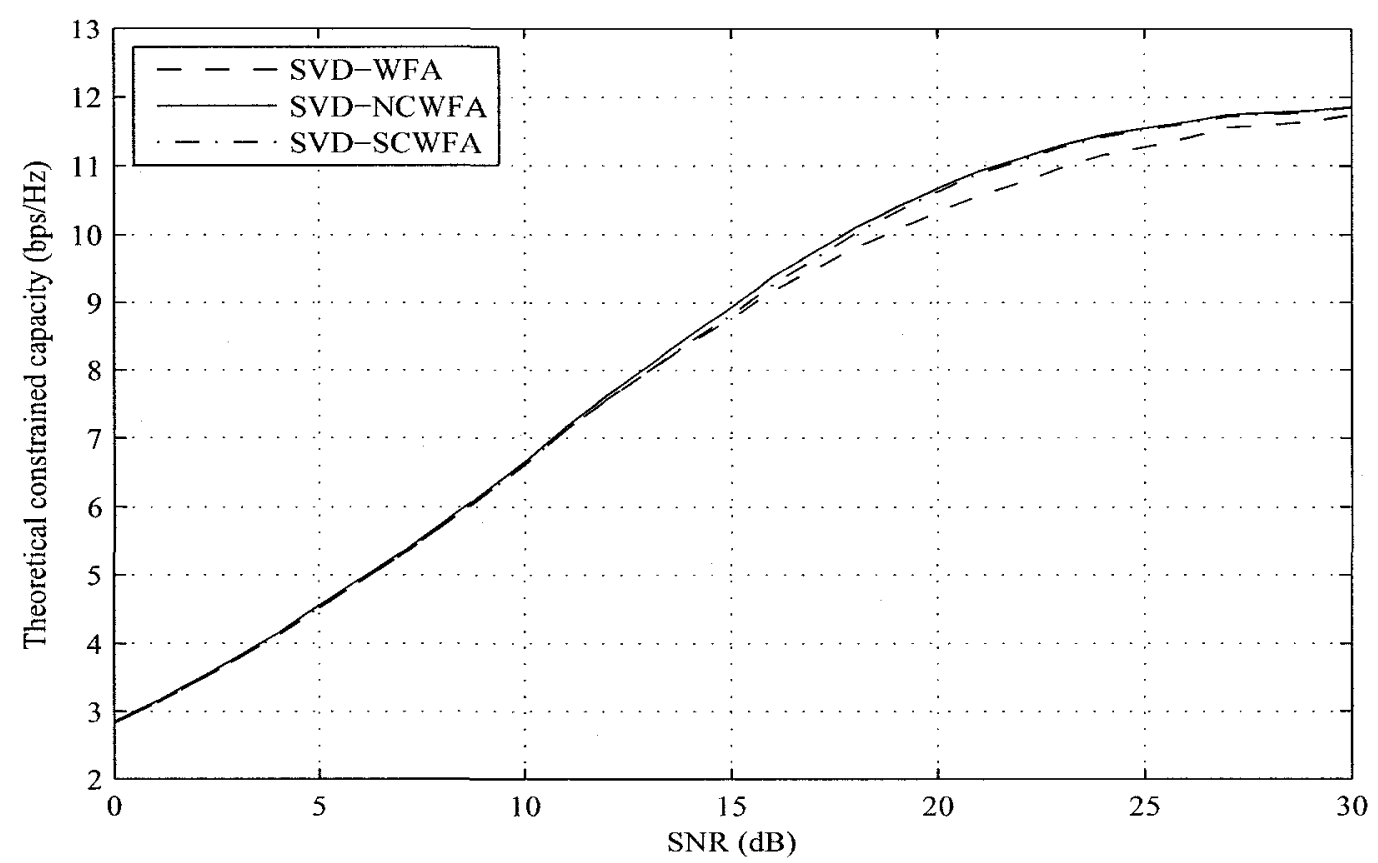

Figure 5.3: Theoretical constrained capacity of $2 \times 2$ SVD-based transceivers using different power and bit-rate allocation algorithms.

complex SCWFA. As a result, we implement the SCWFA in all simulations for the remainder of this thesis. From this point on, we refer to the SCWFA simply as CWFA.

Figures 5.4 and 5.5 give the theoretical constrained capacities of the SVD-based transceivers for the $3 \times 3$ and $4 \times 4$ MIMO systems. The trend seen here is the same as was in Figure 5.3; the CWFA performs better than the regular WFA at higher SNRs. There is a gain in spectral efficiency for the CWFA over the WFA of about $0.45 \mathrm{bps} / \mathrm{Hz}$ and $0.5 \mathrm{bps} / \mathrm{Hz}$ at a SNR of $20 \mathrm{~dB}$ for the $3 \times 3$ and $4 \times 4$ transceivers respectively.

Also introduced in this chapter is a new antenna selection algorithm for the ASGMD transceiver. The CCAS selects the appropriate number of subchannels to transmit on using the constrained capacity as opposed to the unconstrained capacity-based 


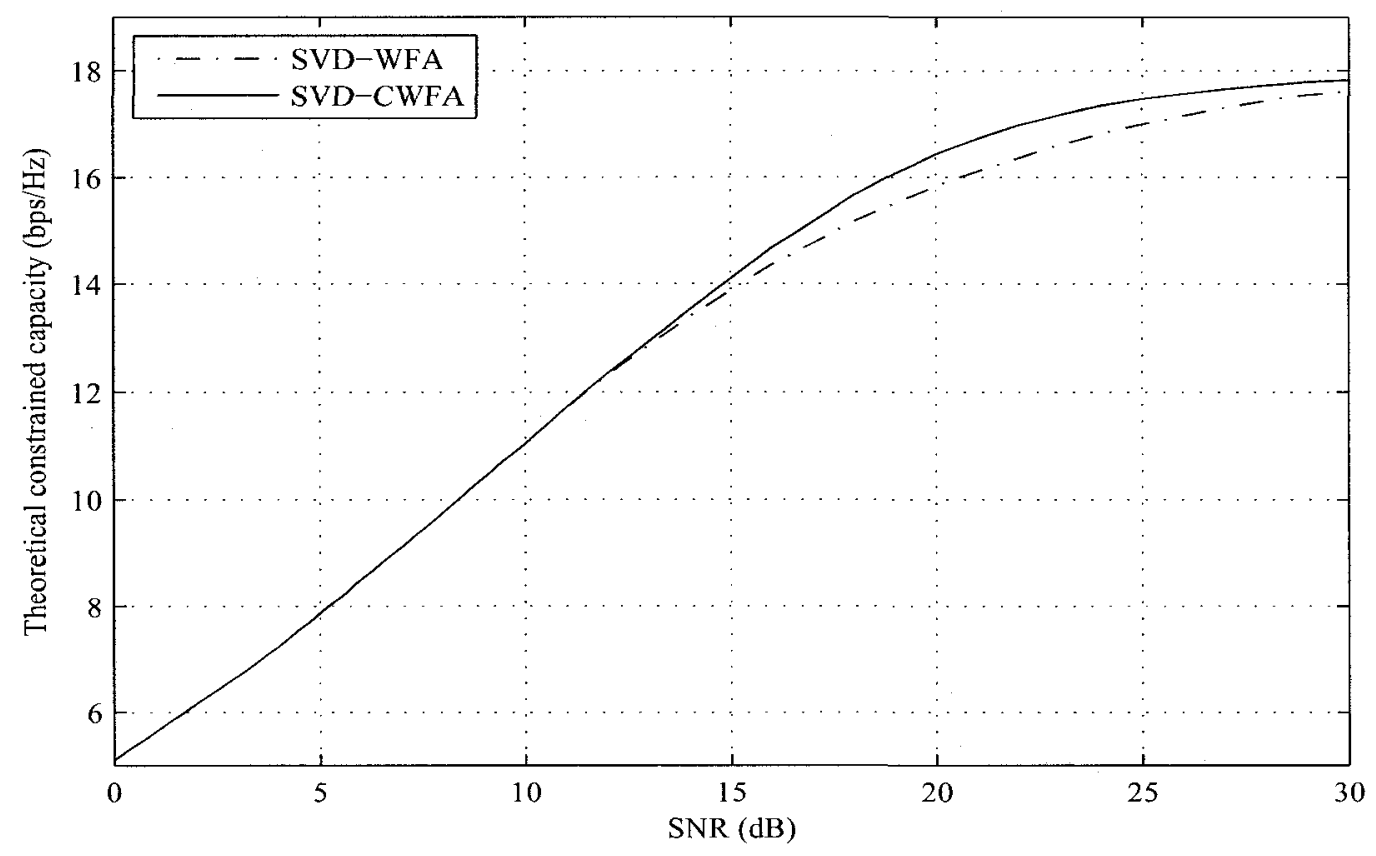

Figure 5.4: Theoretical constrained capacity of $3 \times 3$ SVD-based transceivers using different power and bit-rate allocation algorithms.

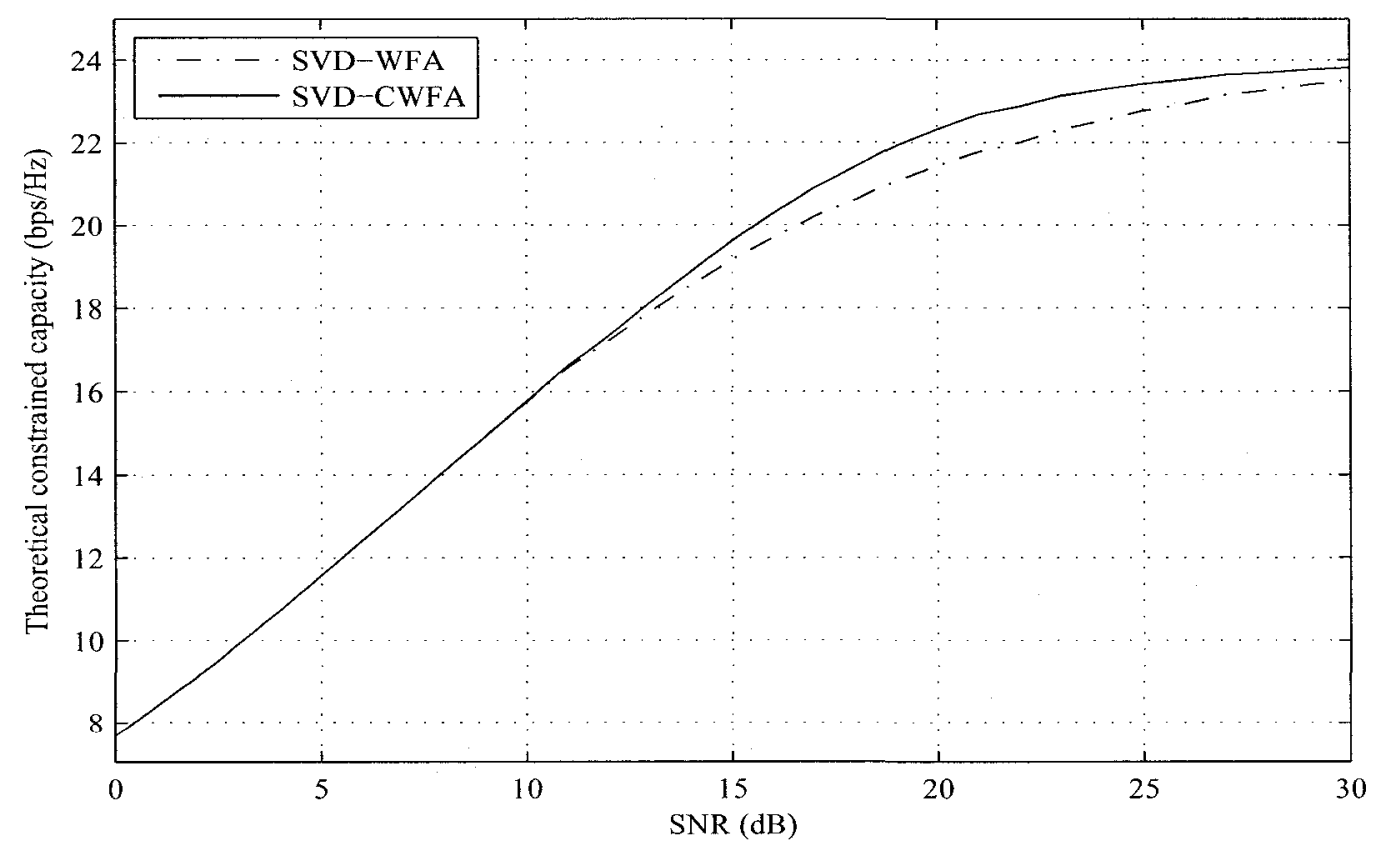

Figure 5.5: Theoretical constrained capacity of $4 \times 4$ SVD-based transceivers using different power and bit-rate allocation algorithms. 


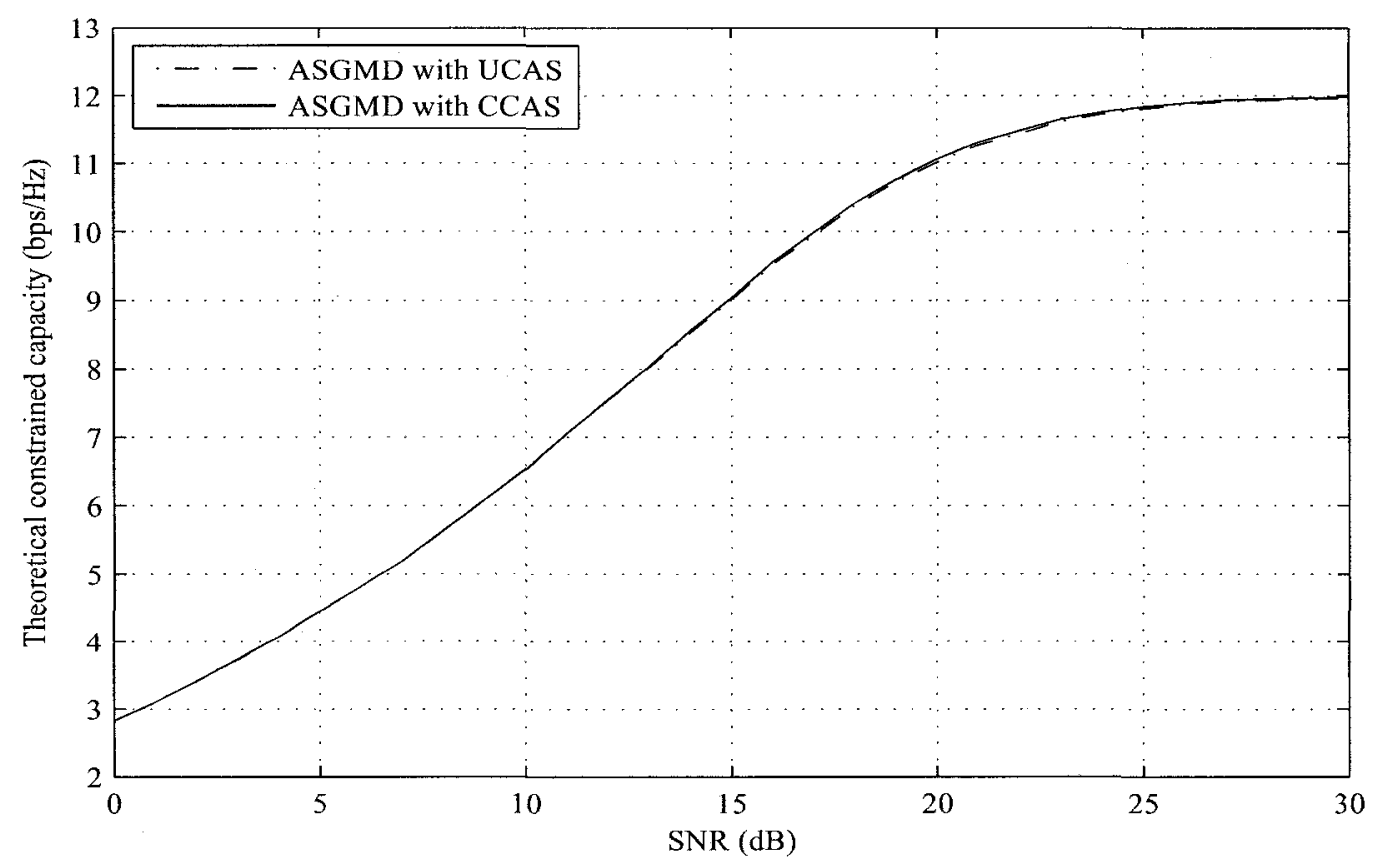

Figure 5.6: Theoretical constrained capacity of $2 \times 2$ ASGMD-based transceivers using different antenna selection algorithms.

antenna selection algorithm (UCAS) for ASGMD introduced in Chapter 2. Figure 5.6 shows the performance of both antenna selection algorithms. While CCAS outperforms UCAS at higher SNRs the loss for using UCAS in a $2 \times 2$ MIMO system is not that significant. Figure 5.7 gives the theoretical constrained throughput performance of $3 \times 3$ ASGMD transceivers. Again, CCAS outperforms UCAS at higher SNRs, but for the loss in throughput performance is more significant than it is for the $2 \times 2$ transceivers. At a SNR of $20 \mathrm{~dB}$ the gain in spectral efficiency of CCAS over UCAS is about $0.1 \mathrm{bps} / \mathrm{Hz}$. Figure 5.8 gives the theoretical constrained throughput performance of $4 \times 4$ ASGMD transceivers. CCAS outperforms UCAS at higher SNRs and an even greater gain is seen for the larger antenna array. At a SNR of $20 \mathrm{~dB}$ the gain in spectral efficiency of CCAS over UCAS is about $0.2 \mathrm{bps} / \mathrm{H} \%$. The results of Figures 5.6 - 5.8 show that there is a small throughput gain in using CCAS instead of UCAS for the ASGMD transceiver. These results also show that the throughput 


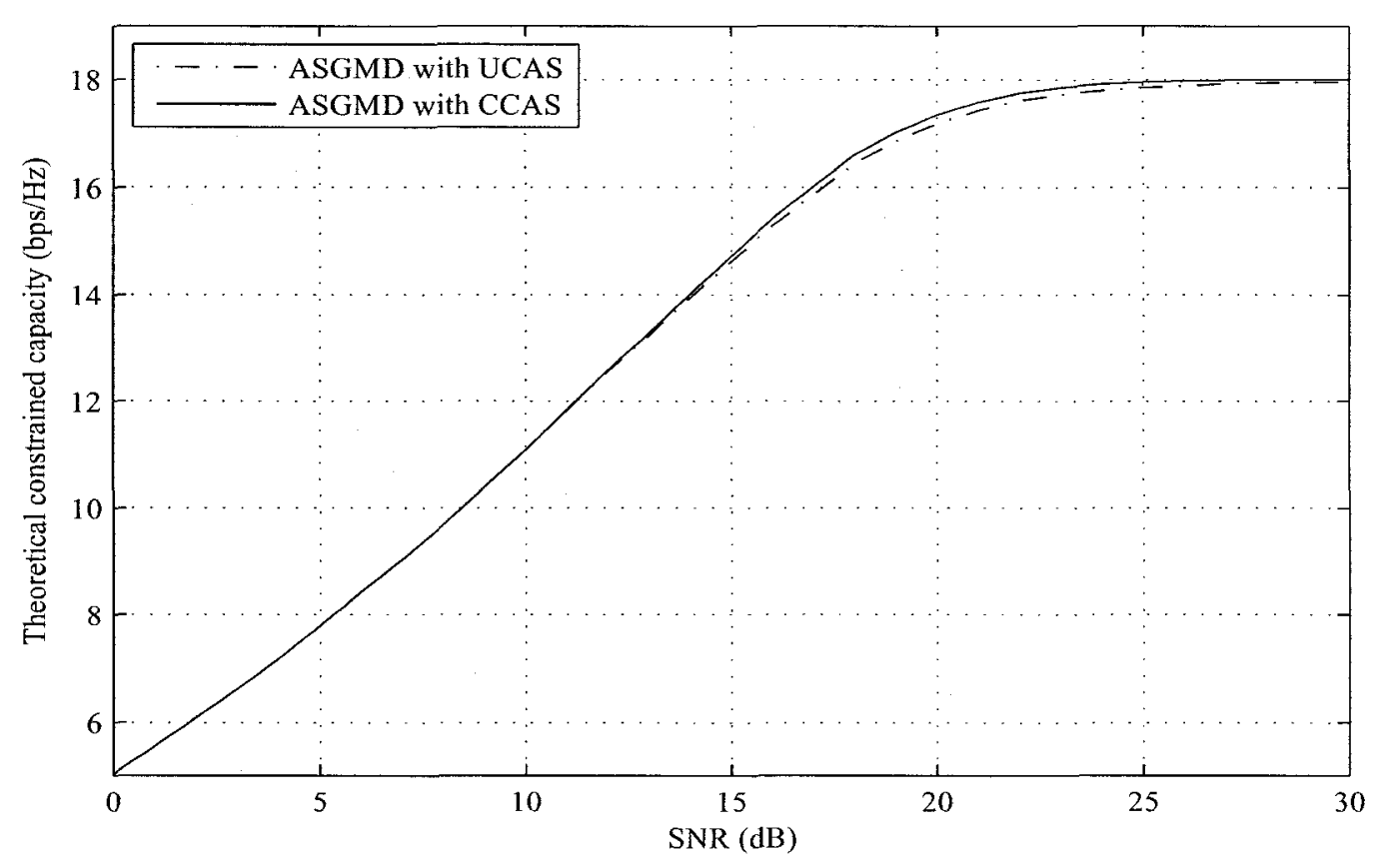

Figure 5.7: Theoretical constrained capacity of $3 \times 3$ ASGMD-based transceivers using different antenna selection algorithms.

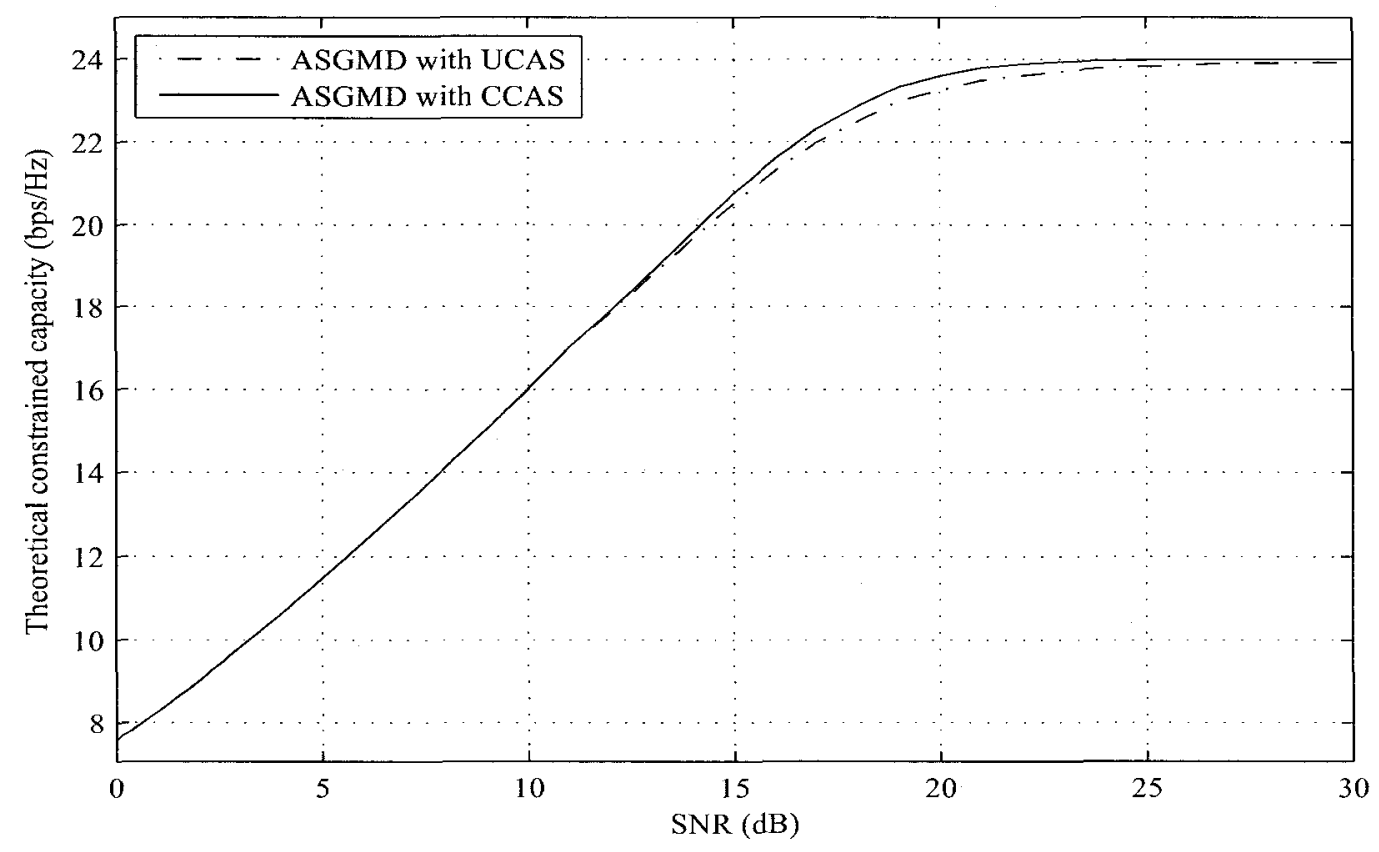

Figure 5.8: Theoretical constrained capacity of $4 \times 4$ ASGMD-based transceivers using different antenna selection algorithms. 
gain increases with the number of antennas used. All simulations from this point on in this thesis use CCAS for antenna selection.

\subsubsection{Experimental Throughput Performance of RC/QC- MIMO with CWFA}

In this chapter we introduced a suboptimal CWFA to do bit-rate and power allocation and CCAS to do antenna selection for the ASGMD transceiver. In Section 5.3.1 the power-allocation portion of the CWFA and the CCAS of ASGMD where evaluated using the theoretical constrained throughput. In this subsection we demonstrate the benefits of the bit-rate allocation portion of CWFA. All evaluated RC/QC-MIMO systems use the CWFA to do bit-rate allocation, and all systems except RC/QC-SVD-CWFA do not use the power allocation portion of the CWFA. The assumptions for the detector of Chapter 4 remain the same. All results are collected for $L_{z}=3$.

Figure 5.9 gives the experimental throughput performance of the $2 \times 2 \mathrm{RC} / \mathrm{QC}$ MIMO transceivers. The same behaviour is displayed by the MIMO transceivers as was shown in Figure 4.11. The SVD-based transceivers dominate at mid-range SNRs on the virtue that they do not have to resolve any MAI. The GMD-based and UCD transceivers have a "dip" at mid-range SNRs as they are not as able to resolve the MAI that comes with larger constellation sizes. An improvement in performance is seen for RC/QC-SVD-CWFA, which outperforms RC/QC-SVD. This was not the case in Chapter 4 when RC/QC-SVD-WFA made use of the regular WFA. This result shows that the theoretical increase in performance seen for SVD using the CWFA also carries over into the experimental results. The performance of the RC/QC-MIMO transceivers that use only the bit-rate allocation portion of 


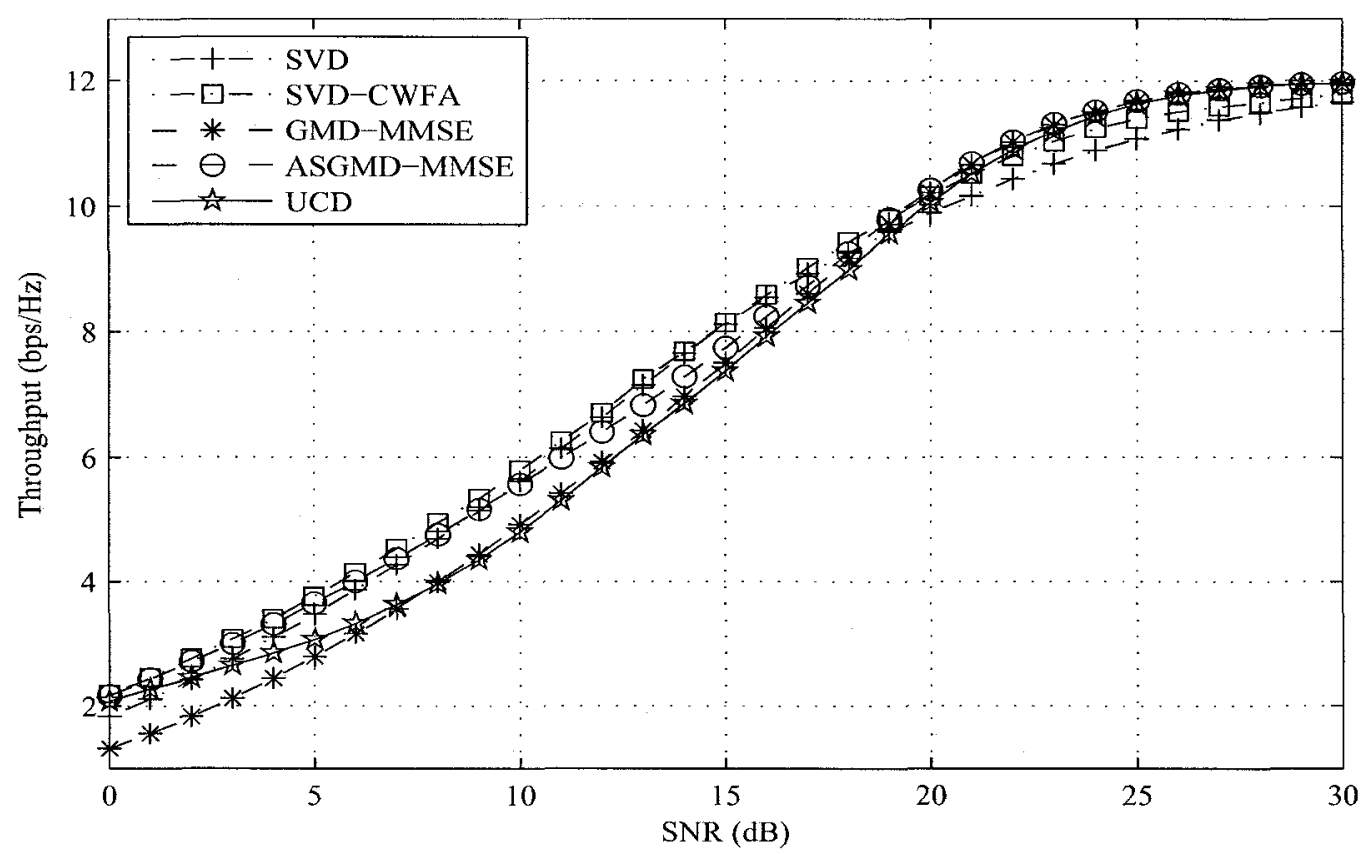

Figure 5.9: Experimental throughput of $2 \times 2 \mathrm{RC} / \mathrm{QC}-\mathrm{MIMO}$ transceivers with different transceiver designs.

the CWFA are shown to perform better than when it is not used; especially at low SNRs. For example, the spectral efficiency of RC/QC-SVD at SNRs of $5 \mathrm{~dB}, 15 \mathrm{~dB}$ and $20 \mathrm{~dB}$ in Chapter 4 is $3.18 \mathrm{bps} / \mathrm{Hz}, 7.79 \mathrm{bps} / \mathrm{Hz}$ and $10.15 \mathrm{bps} / \mathrm{Hz}$ respectively; whereas it is $3.47 \mathrm{bps} / \mathrm{Hz}, 8.12 \mathrm{bps} / \mathrm{Hz}$ and $9.89 \mathrm{bps} / \mathrm{Hz}$ respectively, in Chapter 5. As another example, the spectral efficiency of RC/QC-GMD in Chapter 4 at those same SNRs is $2.6 \mathrm{bps} / \mathrm{Hz}, 7.28 \mathrm{bps} / \mathrm{Hz}$ and $10.15 \mathrm{bps} / \mathrm{Hz}$ respectively; whereas it is $2.79 \mathrm{bps} / \mathrm{Hz}, 7.5 \mathrm{bps} / \mathrm{Hz}$ and $10.21 \mathrm{bps} / \mathrm{Hz}$ respectively, in Chapter 5. Since the RC/QC-MIMO systems of Chapter 4 can only use 64-QAM constellations, it is more likely that there will be error propagation in the layered detector than for the adaptable CWFA, which chooses constellation sizes that are appropriate for the SINRs of each subchannel. This has a larger impact at lower SNRs because of the 64-QAM constellation's poor low-SNR performance, as was demonstrated in Section 3.3. Though the low-SNR improvement is more noticeable, all $2 \times 2 \mathrm{RC} / \mathrm{QC}-\mathrm{MIMO}$ 


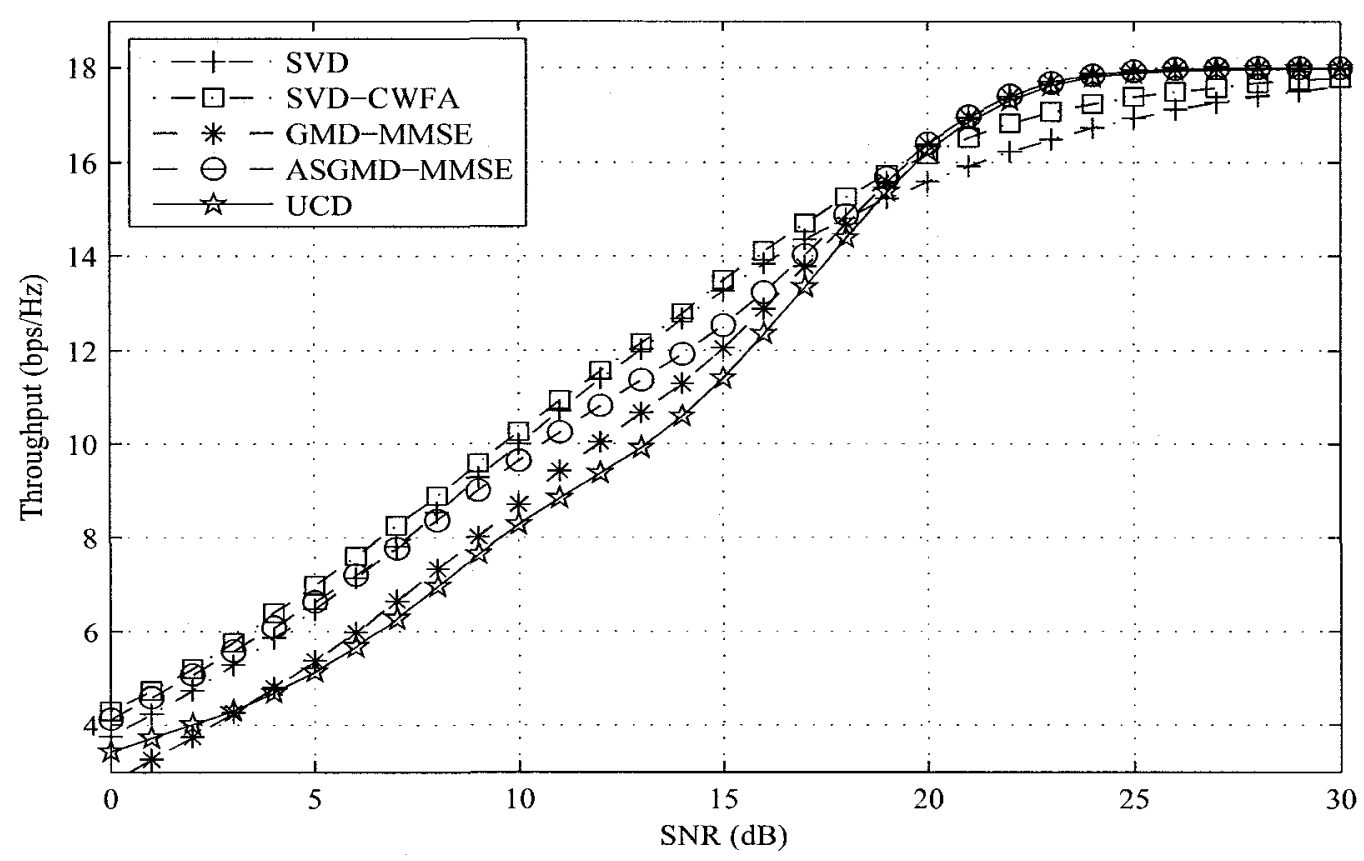

Figure 5.10: Experimental throughput of $3 \times 3 \mathrm{RC} / \mathrm{QC}-\mathrm{MIMO}$ transceivers with different transceiver designs.

systems have better throughput performance, for all SNRs, with the bit-rate allocation algorithm of the CWFA.

Figure 5.9 demonstrates the throughput performance advantage that the RC/QC-ASGMD with CCAS has over the RC/QC-ASGMD with UCAS The spectral efficiency of RC/QC-ASGMD at $5 \mathrm{~dB}, 15 \mathrm{~dB}$ and $25 \mathrm{~dB}$ in Chapter 4 is 3.44 bps $/ \mathrm{Hz}, 7.65 \mathrm{bps} / \mathrm{Hz}$ and $11.6 \mathrm{bps} / \mathrm{Hz}$; whereas it is $3.65 \mathrm{bps} / \mathrm{Hz}, 7.75 \mathrm{bps} / \mathrm{Hz}$ and $11.68 \mathrm{bps} / \mathrm{Hz}$ respectively, in Chapter 5. Figure 5.9 also shows that unlike UCAS RC/QC-ASGMD, CCAS RC/QC-ASGMD is able to converge faster with maximum nominal rate.

Figures 5.10 and 5.11 give the experimental throughput performance of the $3 \times 3$ and $4 \times 4$ RC/QC-MIMO transceivers respectively. Figures 5.10 and 5.11 dis- 


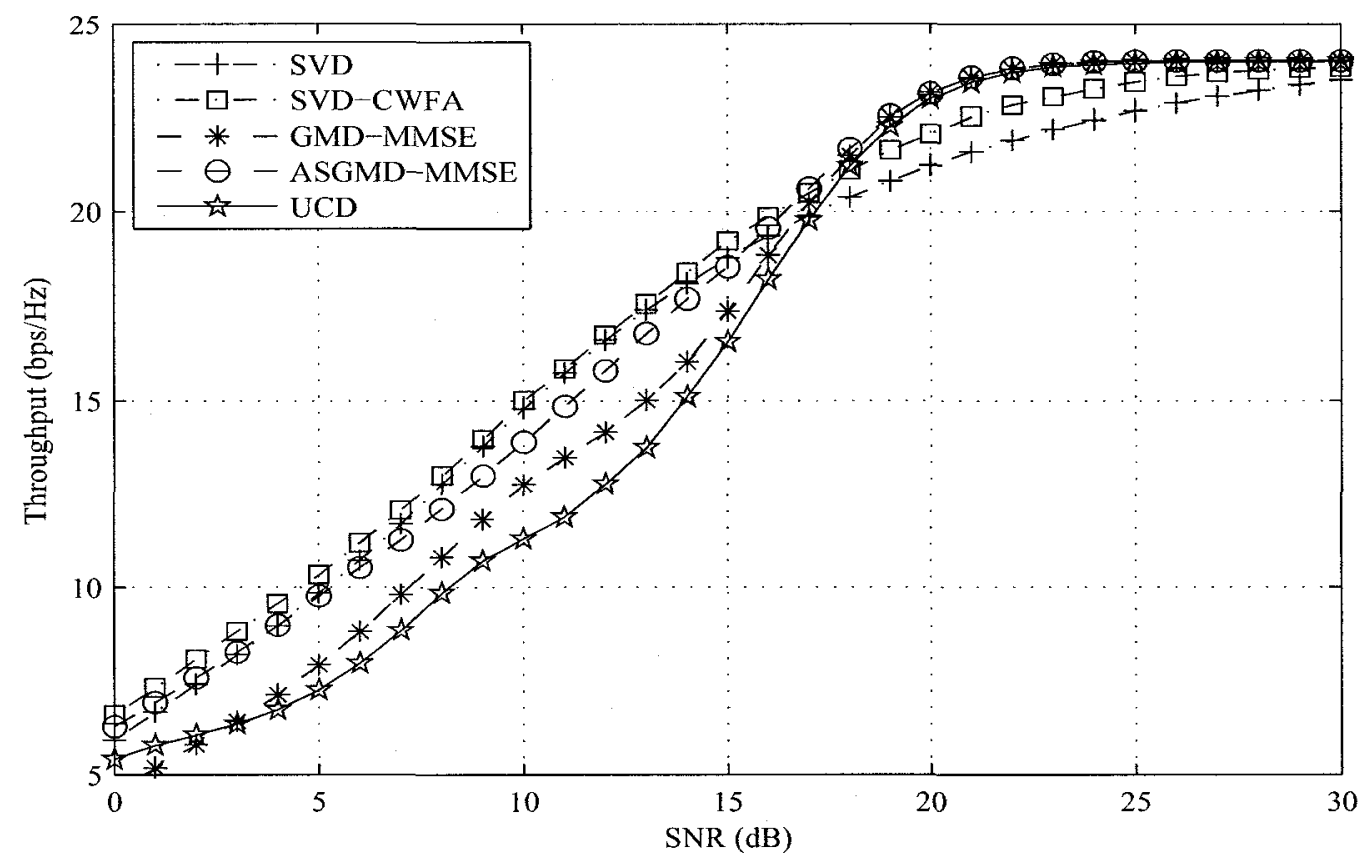

Figure 5.11: Experimental throughput of $4 \times 4 \mathrm{RC} / \mathrm{QC}-\mathrm{MIMO}$ transceivers with different transceiver designs.

play the same behavior discussed for Figure 5.9, in that throughput performance gains are more noticeable at low SNRs, RC/QC-SVD-CWFA has better performance than RC/QC-WFA and CCAS RC/QC-ASGMD is able to converge faster with the system's maximum nominal rate. 


\section{Chapter 6}

\section{Tunable Soft-Symbol Detection for Layered BLAST Detection}

The detector of Chapter 4 creates soft-symbol and LLR estimates with the assumption that the residual MAI, left uncancelled in the decision variable, is Gaussian. Given the actual symbol statistics and the law of large numbers this is may be a good assumption when a sufficiently large number of transmit antennas and constellation size is used; however, especially for smaller constellation sizes and antenna arrays, modelling the residual MAI as a Gaussian random variable is not precise. In this chapter we derive a SS/LLRE based on the probability density function (pdf) of the MAI of all interferers. In addition, we design an adaptable SS/LLRE that considers the statistics of a subset of the MAI, while assuming the MAI outside that subset is a Gaussian random variable. The latter of the two SS/LLREs is then evaluated in terms of its BER and experimental throughput performance, as well as its computational complexity. 


\subsection{The Fully-Tuned SS/LLRE}

In this section we derive a SSE and LLRE using the pdf statistics of the residual MAI for any given detector iteration. The soft-symbol estimates, $\left\{\bar{s}_{i}\right\}$, and variances, $\left\{\sigma_{s_{i}}^{2}\right\}$, are obtained during the layered detection portion of the detector described in Section 4.1 and are used to update the statistics of each symbol "on the fly". The LLRE gives the estimates for the LCUs of Figure 4.1. A SSE creates a symbol estimate by using the most up-to-date statistics on the desired symbol, the MAI and the Gaussian noise. The estimate of the symbol is its expected value. We start by re-organizing (4.5) component-wise as

$$
\begin{aligned}
z_{i} & =\mathbf{d}_{i}\left(\mathbf{y}-\sum_{j=1, j \neq i}^{L} \mathbf{g}_{j} \hat{s}_{j}\right) \\
& =\mathbf{d}_{i} \mathbf{g}_{i} s_{i}+\mathbf{d}_{i} \sum_{j=1, j \neq i}^{L} \mathbf{g}_{j}\left(s_{j}-\bar{s}_{j}\right)+\mathbf{d}_{i} \nu \\
& =t_{i} s_{i}+\sum_{j=1, j \neq i}^{L} t_{j}\left(s_{j}-\bar{s}_{j}\right)+\tilde{\nu}_{i}
\end{aligned}
$$

where $t_{j}$ is a constant that results from the product of vectors $\mathbf{d}_{i}$ and $\mathbf{g}_{j}$; and $\tilde{\nu}_{i}$ is the filtered noise. We see that $z_{i}$ is composed of three components: the desired symbol; a random variable composed of the noise and MAI; and a deterministic component used in cancelling the MAI. We rewrite (6.1) as

$$
z_{i}=t_{i} s_{i}+q_{i}-a_{i}
$$

where $q_{i}$ is a random variable representing the MAI and noise

$$
\begin{aligned}
q_{i} & =\sum_{j=1, j \neq i}^{L} t_{j} s_{j}+\tilde{\nu}_{i} \\
& =I_{i}+\nu_{i}
\end{aligned}
$$

and $a_{i}$ is a deterministic variable based upon the current MAI estimates

$$
a_{i}=\sum_{j=1, j \neq i}^{L} t_{j} \bar{s}_{j} .
$$


Let $\tilde{\mathbf{s}}_{i, l}=\left[\tilde{s}_{1, l}, \ldots, \tilde{s}_{i-1, l}, \tilde{s}_{i+1, l}, \ldots, \tilde{s}_{L, l}\right]$ be one possible realizations of $\mathbf{s}_{i}=$ $\left[s_{1}, \ldots, s_{i-1}, s_{i+1}, \ldots, s_{L}\right]$. There are $N_{e}=\prod_{j=1, j \neq i}^{L} M_{j}$ possible realizations in total. Because the transmitted symbols are independent, the probability that $\tilde{\mathbf{s}}_{i, l}$ was transmitted is

$$
\operatorname{Pr}\left\{\mathbf{s}_{i}=\tilde{\mathbf{s}}_{i, l}\right\}=\prod_{j=1, j \neq i}^{L} \operatorname{Pr}\left\{s_{j}=\tilde{s}_{j, l}\right\}
$$

The noise, $\tilde{\nu}_{i}$, is Gaussian with pdf

$$
f_{\tilde{\nu}_{i}}\left(\tilde{\nu}_{i}\right)=\frac{1}{\pi \sigma_{\tilde{\nu}_{i}}^{2}} \exp \left(\frac{\left|\tilde{\nu}_{i}\right|^{2}}{\sigma_{\tilde{\nu}_{i}}^{2}}\right)
$$

where $\sigma_{\tilde{\nu}_{i}}^{2}$ is the noise variance. The pdf of $q_{i}$

$$
\begin{aligned}
f_{q_{i}}\left(q_{i}\right) & =\sum_{l=1}^{N_{e}} f_{q_{i}}\left(q_{i} \mid \mathbf{s}_{i}=\tilde{\mathbf{s}}_{i, l}\right) \operatorname{Pr}\left\{\mathbf{s}_{i}=\tilde{\mathbf{s}}_{i, l}\right\} \\
& =\sum_{l=1}^{N_{e}} f_{\tilde{\nu}_{i}}\left(\tilde{\nu}_{i}\right)\left(q_{i}-\chi_{l}\right) \operatorname{Pr}\left\{\mathbf{s}_{i}=\tilde{\mathbf{s}}_{i, l}\right\}
\end{aligned}
$$

where

$$
\chi_{l}=\sum_{j=1, j \neq i}^{L} t_{j} \tilde{s}_{j, l}
$$

From (6.7) the likelihood function of $z_{i}$ given $s$ was transmitted on subchannel $i$ is defined as

$$
\begin{aligned}
f_{z_{i}}\left(z_{i} \mid s_{i}=s\right) & =f_{q_{i}}\left(z_{i}-t_{i} s+a_{i}\right) \\
& =\sum_{l=1}^{N_{e}} \operatorname{Pr}\left\{\mathbf{s}_{i}=\tilde{\mathbf{s}}_{i, l}\right\} f_{\tilde{\nu}_{i}}\left(z_{i}-t_{i} s-\chi_{l}+a_{i}\right) \\
& =\frac{1}{\pi \sigma_{\tilde{\nu}_{i}}^{2}} \sum_{l=1}^{N_{e}} \operatorname{Pr}\left\{\mathbf{s}_{i}=\tilde{\mathbf{s}}_{i, l}\right\} \exp \left(\frac{\left|z_{i}-t_{i} s-\chi_{l}+a_{i}\right|^{2}}{\sigma_{\tilde{\nu}_{i}}^{2}}\right) .
\end{aligned}
$$

Having (6.8) we can determine the LLRs for each transmitted bit using (4.12). Substituting (4.8) into (4.12) the LLR of a code bit can be found as

$$
\lambda_{i}^{(k)}=\ln \left(\frac{\sum_{s \in \mathcal{S}_{i}} f_{z_{i}}\left(z_{i} \mid s_{i}=s\right) \operatorname{Pr}\left\{s_{i}=s\right\} \operatorname{Pr}\left\{c_{i}^{(k)}=1 \mid s_{i}=s\right\}}{\sum_{s \in \mathcal{S}_{i}} f_{z_{i}}\left(z_{i} \mid s_{i}=s\right) \operatorname{Pr}\left\{s_{i}=s\right\} \operatorname{Pr}\left\{c_{i}^{(k)}=0 \mid s_{i}=s\right\}}\right) .
$$


Furthermore, we assume no a priori knowledge of the desired symbol, $s_{i}$. Thus, we can drop the $\operatorname{Pr}\left\{s_{i}=s\right\}$ factors from (6.9) since these probabilities are all equal and will cancel out of the equation. This gives a new LLR calculation formula, which is

$$
\lambda_{i}^{(k)}=\ln \left(\frac{\sum_{s \in \mathcal{S}_{i}} \sum_{l=1}^{N_{e}} \operatorname{Pr}\left\{c_{i}^{(k)}=1 \mid s_{i}=s\right\} \operatorname{Pr}\left\{\mathbf{s}_{i}=\tilde{\mathbf{s}}_{i, l}\right\} \exp \left(\frac{\left|z_{i}-t_{i} s-\chi_{l}+a_{i}\right|^{2}}{\sigma_{\tilde{\nu}_{i}}^{2}}\right)}{\sum_{s \in \mathcal{S}_{i}} \sum_{l=1}^{N_{e}} \operatorname{Pr}\left\{c_{i}^{(k)}=0 \mid s_{i}=s\right\} \operatorname{Pr}\left\{\mathbf{s}_{i}=\tilde{\mathbf{s}}_{i, l}\right\} \exp \left(\frac{\left|z_{i}-t_{i} s-\chi_{l}+a_{i}\right|^{2}}{\sigma_{\tilde{\nu}_{i}}^{2}}\right)}\right) .
$$

To obtain the symbol estimates we require the probability of the desired symbol being a specific symbol mapping. This can be obtained by substituting (6.8) into (4.8) to get the a posteriori probability of each symbol

$$
\begin{aligned}
\operatorname{Pr}\left\{s_{i}=s \mid z_{i}\right\} & =\frac{1}{K_{z}} f_{z_{i}}\left(z_{i} \mid s_{i}=s\right) \\
& =\frac{1}{K_{z}} \sum_{l=1}^{N_{e}} \operatorname{Pr}\left\{\mathbf{s}_{i}=\tilde{\mathbf{s}}_{i, l}\right\} \exp \left(\frac{\left|z_{i}-t_{i} s-\chi_{k}+a_{i}\right|^{2}}{\sigma_{\bar{\nu}_{i}}^{2}}\right)
\end{aligned}
$$

and using the a posteriori probabilities, a symbol estimate and variance are obtained using (4.3) and (4.4). It is possible to reduce to the total number of computations for (6.10) by splitting it into its real and imaginary portions as is mentioned in Section 4.1.3.

The major drawback to this solution is that the number of computations required to calculate all $N_{e}$ possible realizations of the MAI, $\chi_{l}$, grows exponentially with the size of the constellations used and number of symbols transmitted. In fact, the LLR estimate expressed in (6.10) has computational complexity growth similar to a ML detector. In previous chapters we noted that the ML detector is not practical for systems with medium to high spectral efficiency. Left unchanged, the solution suggested in this section would also not be implementable. The SS/LLRE tunes in to the MAI in order to cancel it, so we call it a tunable SS/LLRE. A SS/LLRE that considers all tones of the MAI is called a Fully-Tuned (FT-SS/LLRE). 


\subsection{Tunable SS/LLRE}

To reduce the number of tones considered we allow the Tunable SS/LLRE the ability to make some assumptions about the statistics of some of the interferers, while still retaining their contribution to the total MAI. In this section we design a method that reduces the number of MAI realizations considered by modelling a subset of interferers as a Gaussian random variable.

Largest interferers tuning A strategy for reducing the number of tones considered is to pick the $N_{I}$ largest interferers contributing to the MAI at a given detection layer. Let $\mathcal{C}$ be the subset of largest interferers. In this case, we regard all the MAI components that are not considered part of subset $\mathcal{C}$ as Gaussian noise. Restructuring (6.3), $q_{i}$ is decomposed as follows

$$
\begin{aligned}
q_{i} & =\sum_{j \in \mathcal{C}} t_{j} s_{j}+\sum_{j \notin \mathcal{C}} t_{j} s_{j}+\nu_{i} \\
& =I_{i}+\sum_{j \notin \mathcal{C}} t_{j} s_{j}+\nu_{i}
\end{aligned}
$$

so that now $I_{i}$ is based upon the subset $\mathcal{C}$, or the set of largest interferers. The largest interferers are determined by the residual energy left by any uncancelled post-filtering interference. The energy contributed to the MAI by the $j^{\text {th }}$ interferer is

$$
\varepsilon_{j}=\sigma_{s_{j}}^{2}\left|t_{j}\right|^{2}
$$

The interferer energies are then used in the following rule to determine the set of largest interferers

$$
\mathcal{C}=\arg \max _{j \in \omega} \varepsilon_{j}
$$

where $\omega$ is the set including all interferers that make up the MAI (i.e. $\omega=\{1, \ldots, i-1, i+1, \ldots, L\})$. The interferers in $\mathcal{C}$ make up the random variable, $I_{i}$ (i.e. $I_{i}=\sum_{j \in \mathcal{C}} t_{j} s_{j}$ ), and this variable is used in (6.5) - (6.10) to determine LLRs of each 
bit. The contribution of the remaining $|\omega|-N_{I}$ interferers is determined from the statistics calculated at the current layer of BLAST detection. Since we consider the MAI not in set $\mathcal{C}$ as Gaussian, we need only obtain their variance and mean to model their statistics. The sum of unconsidered MAI and noise is modelled as a Gaussian random variable, $v_{i}$, with zero-mean and a variance $\sigma_{v_{i}}^{2}=\sigma_{\tilde{\nu}_{i}}^{2}+\sum_{j \notin \mathcal{C}} \varepsilon_{j}$. Recognizing that $a_{i}$ of (6.4) already contains the current means of the unconsidered MAI, we can modify $a_{i}$ to only include the interferers that are in the set $\mathcal{C}$. That is,

$$
a_{i}=\sum_{j \in \mathcal{C}} g_{i, j} \bar{s}_{j}
$$

The number of possibilities for the MAI, $N_{e}$, has been reduced to $N_{e}=\prod_{j \in \mathcal{C}} M_{j}$ and the LLRs can be calculated using (6.10), except that the $\tilde{\mathbf{s}}_{i, l}$ used for the T-SS/LLRE only contain symbols corresponding to interferers in $\mathcal{C}$ and $\sigma_{v_{i}}^{2}$ is substituted for $\sigma_{\tilde{\nu}_{i}}^{2}$.

The largest interferer method still has exponential growth with each additional interferer that is added; however, it has the flexibility to reduce or increase the number of considered interferers for each new set of received samples. This is particularly advantageous when adaptive modulation is employed by the transmitter. This means more interference could be tuned into when the bit-rate is relatively low, and fewer tones could be considered when the bit rate is higher. The FT-SS/LLRE described in Section 6.1 is subsumed by the largest interferer method as a special case when $N_{I}=L$. When $N_{I}=0$ T-SS/LLRE is the all Gaussian SS/LLRE of Chapter 4.

We note that we could use many different methods to further reduce the number of computations required for T-SS/LLRE. One example is to use only those possibilities with a probability larger than a certain threshold $P r^{(t h)}$. That is only accept the possible realization of $\tilde{\mathbf{s}}_{i, l}$, if $\operatorname{Pr}\left\{\mathbf{s}_{i}=\tilde{\mathbf{s}}_{i, l}\right\} \geq \operatorname{Pr}{ }^{(t h)}$. The computational complexity 
of T-SS/LLRE can be further reduced by recognizing that for the case where no $a$ priori information is available about an interferer, that specific interferer should be ignored when building set $\mathcal{C}$. This reduces the number of considered interferers on the first iteration of our detector. For example, on the first detection layer of the first iteration the set of possible interferers is always zero.

\subsection{BER Performance of the MMSE-ISF Detector with T-SS/LLRE}

The previous two section discussed methodologies for creating soft-symbol and LLR estimates from decision variables containing MAI. This section is dedicated to evaluating the BER performance of an uncoded MIMO system implementing the MMSE-ISF detector introduced in Chapter 4 with no ECC and a T-SS/LLRE. We use the MMSE-SIC detector with HSE to provide a benchmark with which to compare the gains of the MMSE-ISF T-SS/LLRE detector for a variety of antenna array sizes using 16-QAM and 64-QAM constellations. The T-SS/LLRE is implemented with $\operatorname{Pr}^{(t h)}=10^{-10}$, and only considering interferers for which a priori information is already known.

Figure 6.1 gives the BER performance of the $2 \times 2$ MIMO system using a 16-QAM constellation. The performance gain of the MMSE-ISF using a T-SS/LLRE with $N_{I}=0$ over the MMSE-SIC detector is evident even on the first iteration, though it is very small (roughly $0.3 \mathrm{~dB}$ ). However, when the detector is allowed to continue on to iterations 2 and 3 we see that the SNR performance gain is much larger. On the second iteration the SNR gain is about $1.8 \mathrm{~dB}$ and $1.85 \mathrm{~dB}$ on the third. This 


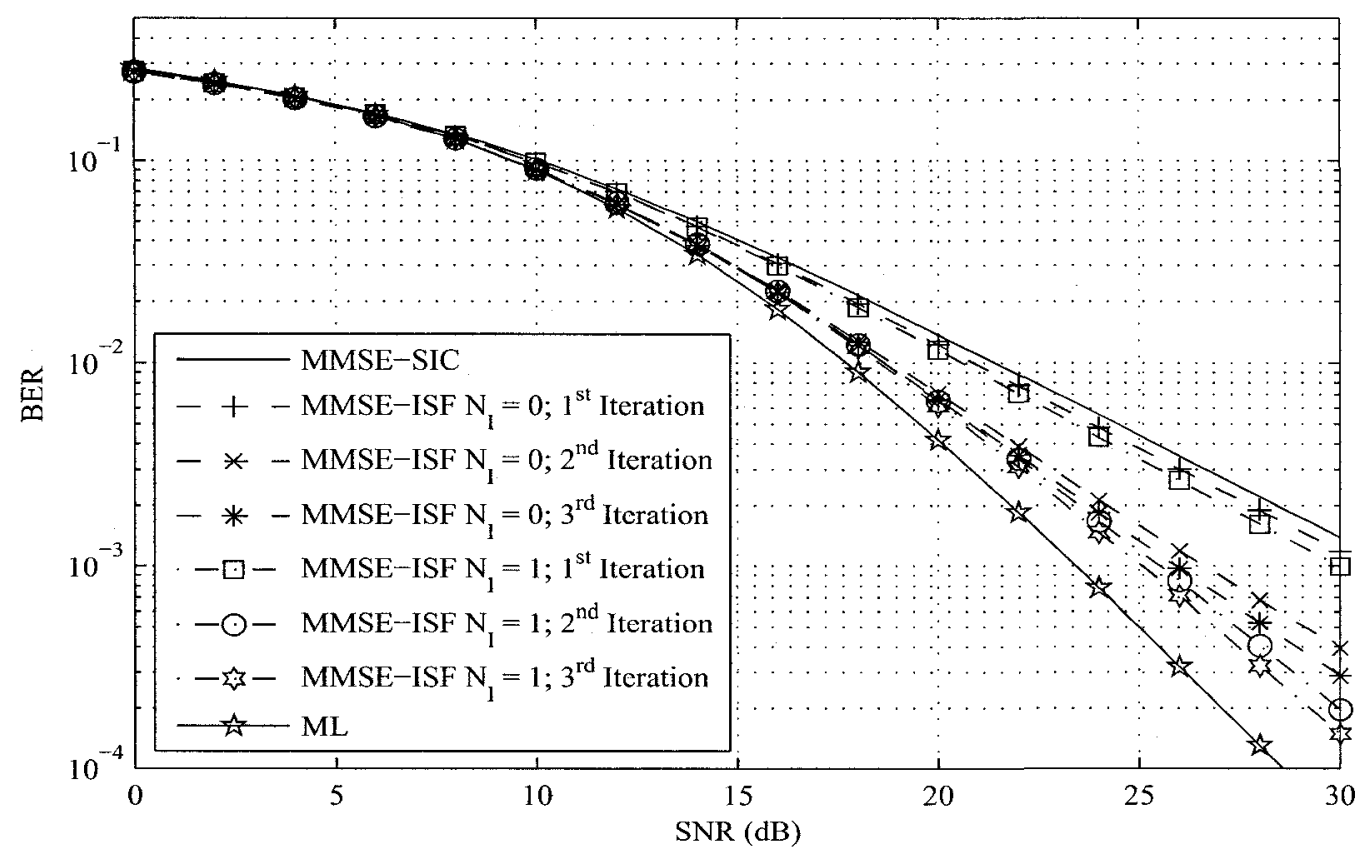

Figure 6.1: BER performance of an uncoded $2 \times 2$ MIMO system using MMSE detectors and 16-QAM.

indicates a diminishing return as we increase the number of iterations. When the T$\mathrm{SS} / \mathrm{LLRE}$ is tuned to $N_{I}=1$ the performance gains between each iteration are even more pronounced. Between the all Gaussian interferer T-SS/LLRE and T-SS/LLRE with $N_{I}=1$ there is an SNR gain of $1.4 \mathrm{~dB}$ at a BER of $2 \times 10^{-3}$ for the first iteration; $4.7 \mathrm{~dB}$ at $10^{-3}$ for the second iteration; and $5 \mathrm{~dB}$ at $10^{-3}$ for the third iteration. Even more impressive is the diversity gain seen by all iterations of the MMSE-ISF for $N_{I}=1$. Even on the first iteration we see that the curve of T-SS/LLRE begins to diverge from the first iteration of the all Gaussian SS/LLRE. This stands to reason, since the $N_{I}=1$ T-SS/LLRE considers up to two symbols simultaneously in detection, much in the same way a ML would for the $2 \times 2 \mathrm{MIMO}$ transceiver.

Figure 6.2 gives the average number of computations that T-SS/LLRE with $N_{I}=1$ must do in order to attain the performance shown in Figure 6.1. It can be 


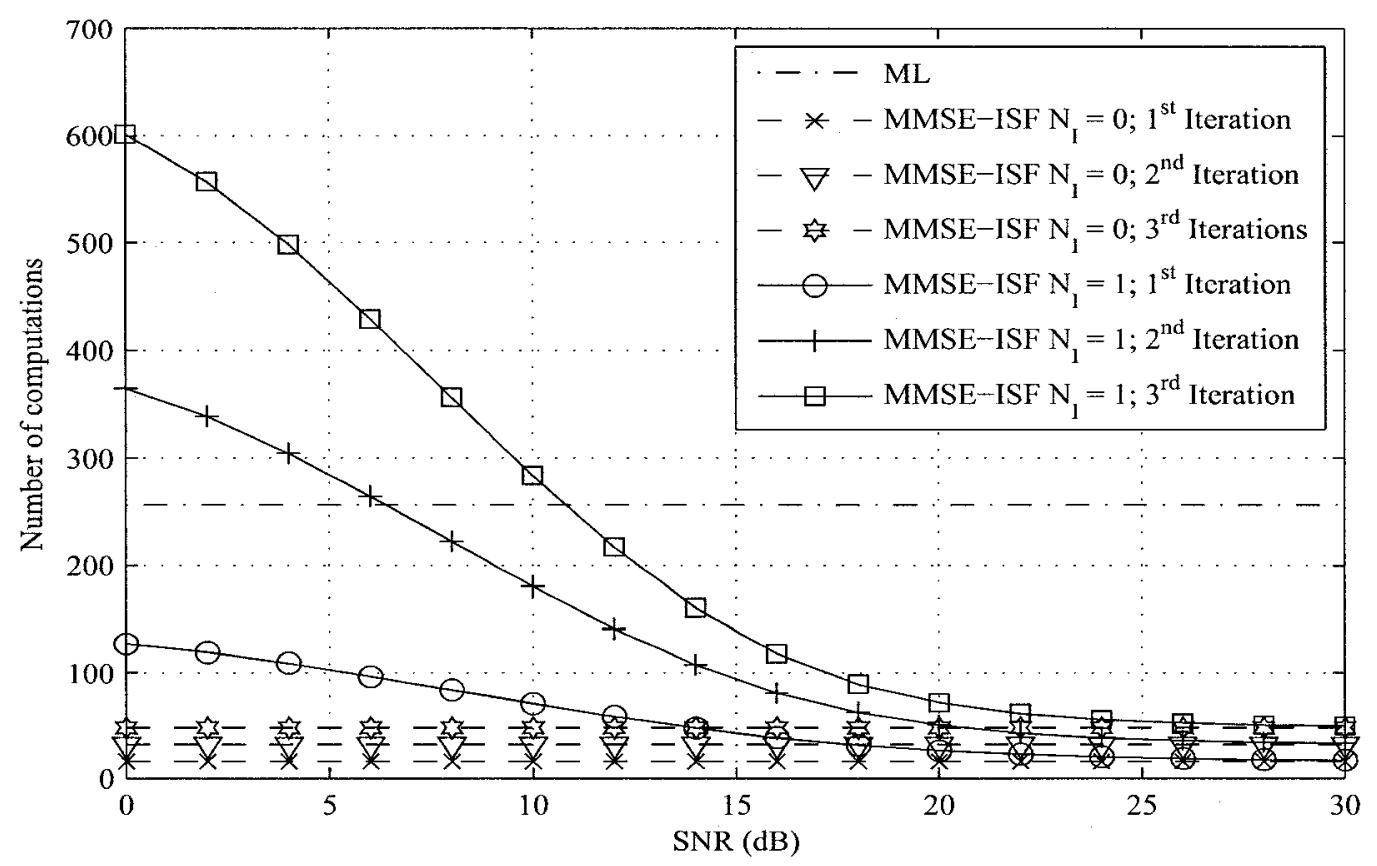

Figure 6.2: Number of exponential computations performed by the $2 \times 2 \mathrm{MIMO}$ system using T-SS/LLRE and MMSE-ISF detector and 16-QAM for $N_{I}=1$.

seen that the first iteration requires considerably less computations than the second and third iterations at lower SNRs. This is due to the fact that in the first layer of the first iteration, no a priori information is known about any interferers. Figure 6.2 demonstrates that for lower SNRs the MMSE-ISF with T-SS/LLRE is more computationally complex than an ML detector, even though it is able to split LLRs into their real and imaginary parts to reduce computational complexity - something that cannot be done with MIMO ML detectors transmitting constellations larger than 4-QAM. Indeed, with the extra computations of nulling vectors this makes the $2 \times 2$ MMSE-ISF using T-SS/LLRE undesirable for $N_{I}=1$. However, for mid-range SNRs and higher SNRs the average number of computations is considerably less than that of an ML detector, which makes its use justifiable. For a probability of error of $10^{-3}$ there is a SNR loss of $1.6 \mathrm{~dB}$ for the third iteration of MMSE-ISF with T-SS/LLRE and $N_{I}=1$ on the ML detector. This BER occurs at $25 \mathrm{~dB}$ for 
the MMSE-ISF. At a SNR of $25 \mathrm{~dB}$ the average total number of computations required of the MMSE-ISF with T-SS/LLRE is about 60 compared to 256 for the ML detector. This is a large reduction in the number of computations and is close to the total number required for T-SS/LLRE with $N_{I}=0$. Furthermore, as was already mentioned, at a BER of $10^{-3}$, T-SS/LLRE with $N_{I}=1$ has a $5 \mathrm{~dB}$ gain on T-SS/LLRE with $N_{I}=0$. In most wireless systems a BER of at least $10^{-3}$ is required to ensure proper operation, which means that we will not likely be operating T-SS/LLRE in those lower SNR ranges. However, should the instantaneous SNR be low, a compromise could be to use the T-SS/LLRE with $N_{I}=0$ since its performance is nearly the same as for T-SS/LLRE with $N_{I}=1$. This is the strength of T-SS/LLRE: adaptability. Being adaptable means that T-SS/LLRE can consider the number of interferers necessary to compute LLRs giving good performance, while still keeping computational complexity relatively low.

Figures 6.3 and 6.4 show the BER performance of T-SS/LLRE for $3 \times 3$ and $4 \times 4$ MIMO transceivers using 16-QAM respectively. For Figure 6.3 we see that for $N_{I}=0$ and one iteration there is already a $0.6 \mathrm{~dB}$ gain on MMSE-SIC. This is a greater difference than when the $2 \times 2$ transceiver was used, and can be explained by the fact that there are more layers in which error propagation can occur, meaning that a ISF detector will add less MAI when an incorrect decision is made than a SIC detector. At a BER of $10^{-3}$ there is an SNR gain of $2.6 \mathrm{~dB}$ from the first iteration to the third iteration of the MMSE-ISF using T-SS/LLRE with $N_{I}=0$. T-SS/LLRE with $N_{I}=1$ with has an SNR gain of $7 \mathrm{~dB}$ over the first iteration of T-SS/LLRE with $N_{I}=0$. These gains are much larger than those made with the $2 \times 2$ transceiver. Figure 6.4 shows the BER performance for T-SS/LLRE for a larger range of $N_{I}$. This is meant to show the benefits of increasing the number of interferers considered by T-SS/LLRE. When $N_{I}=0$ the SNR gain of the MMSE-ISF using T-SS/LLRE 


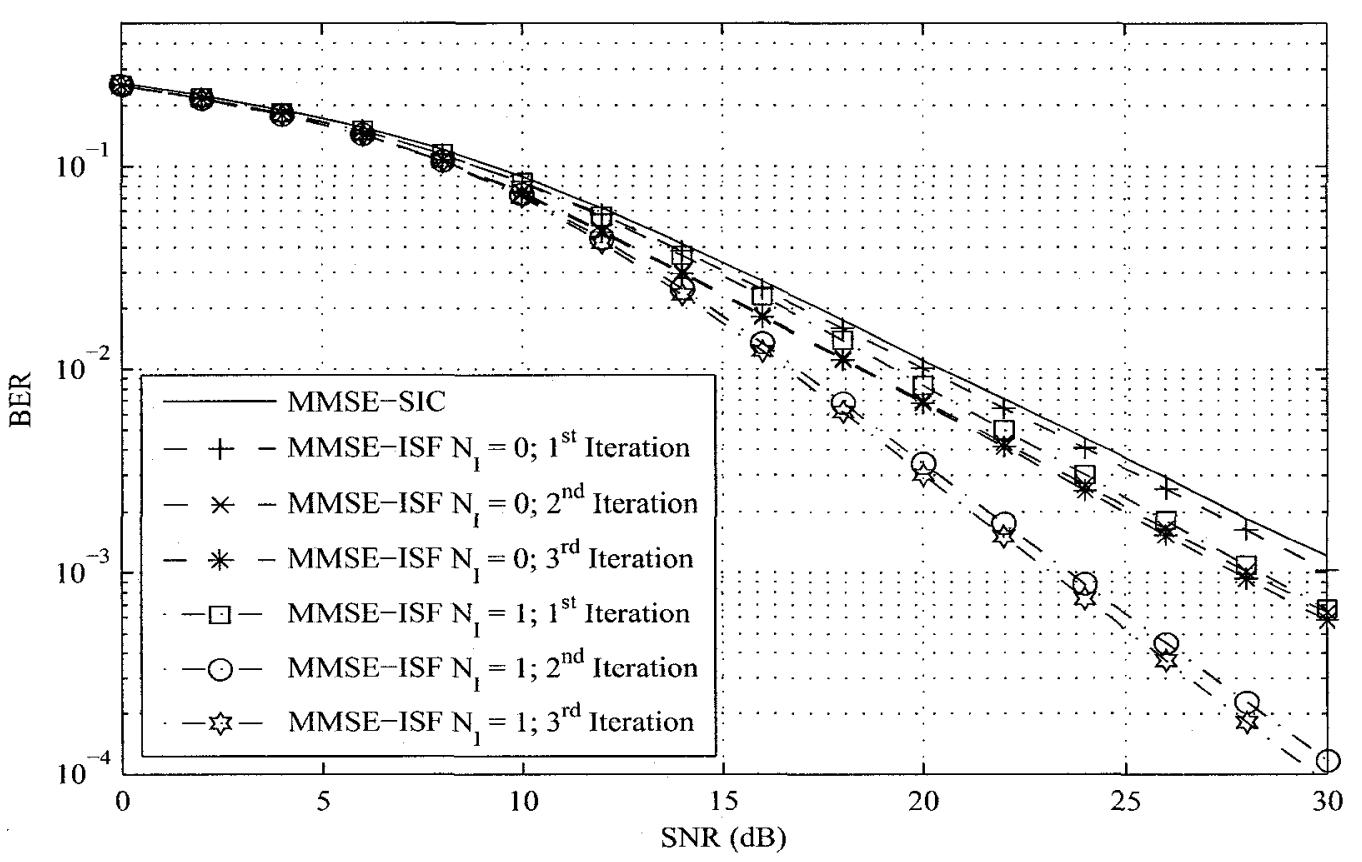

Figure 6.3: BER performance of an uncoded $3 \times 3$ MIMO system using MMSE detectors and 16-QAM.

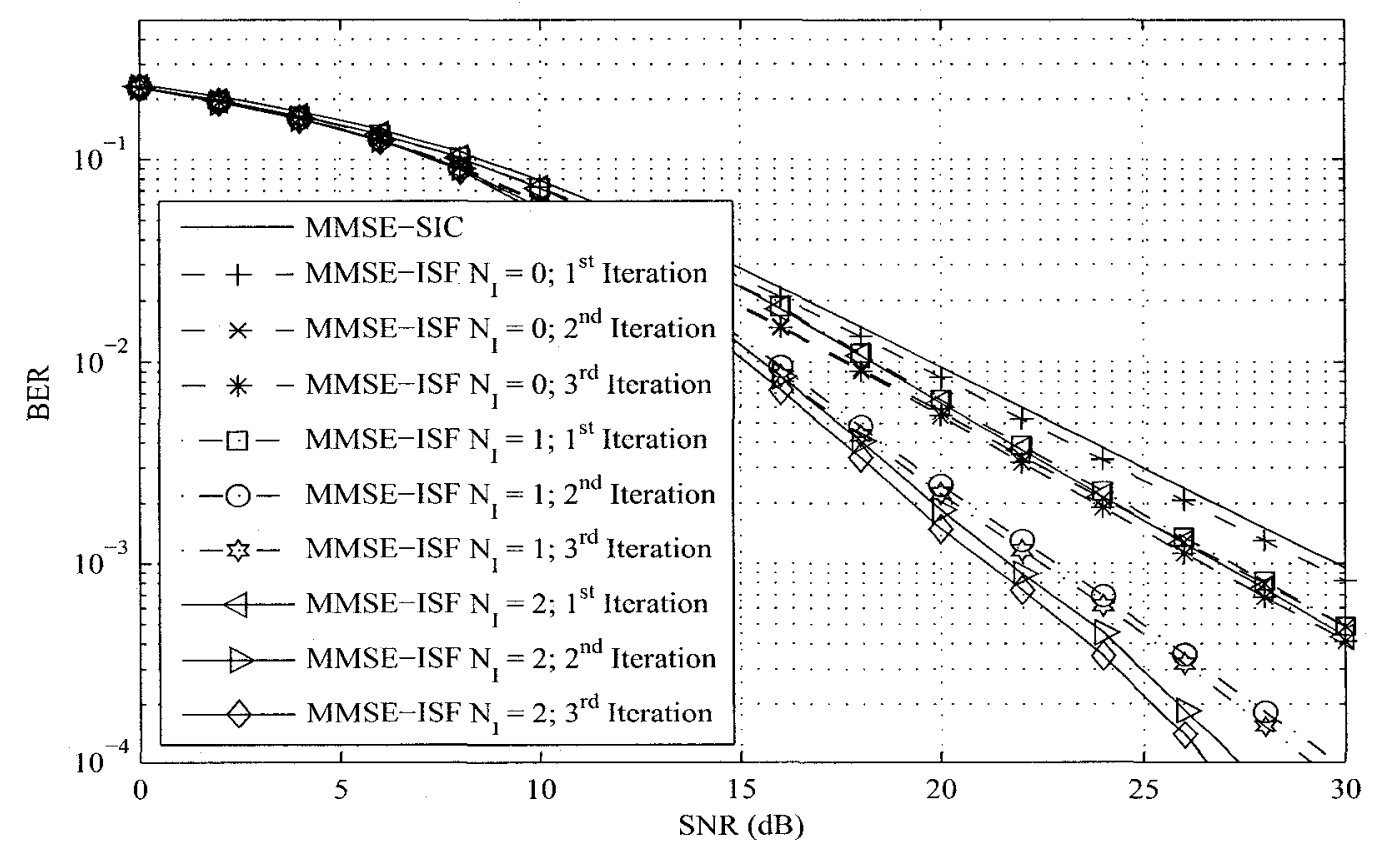

Figure 6.4: BER performance of an uncoded $4 \times 4$ MIMO system using MMSE detectors and 16-QAM. 


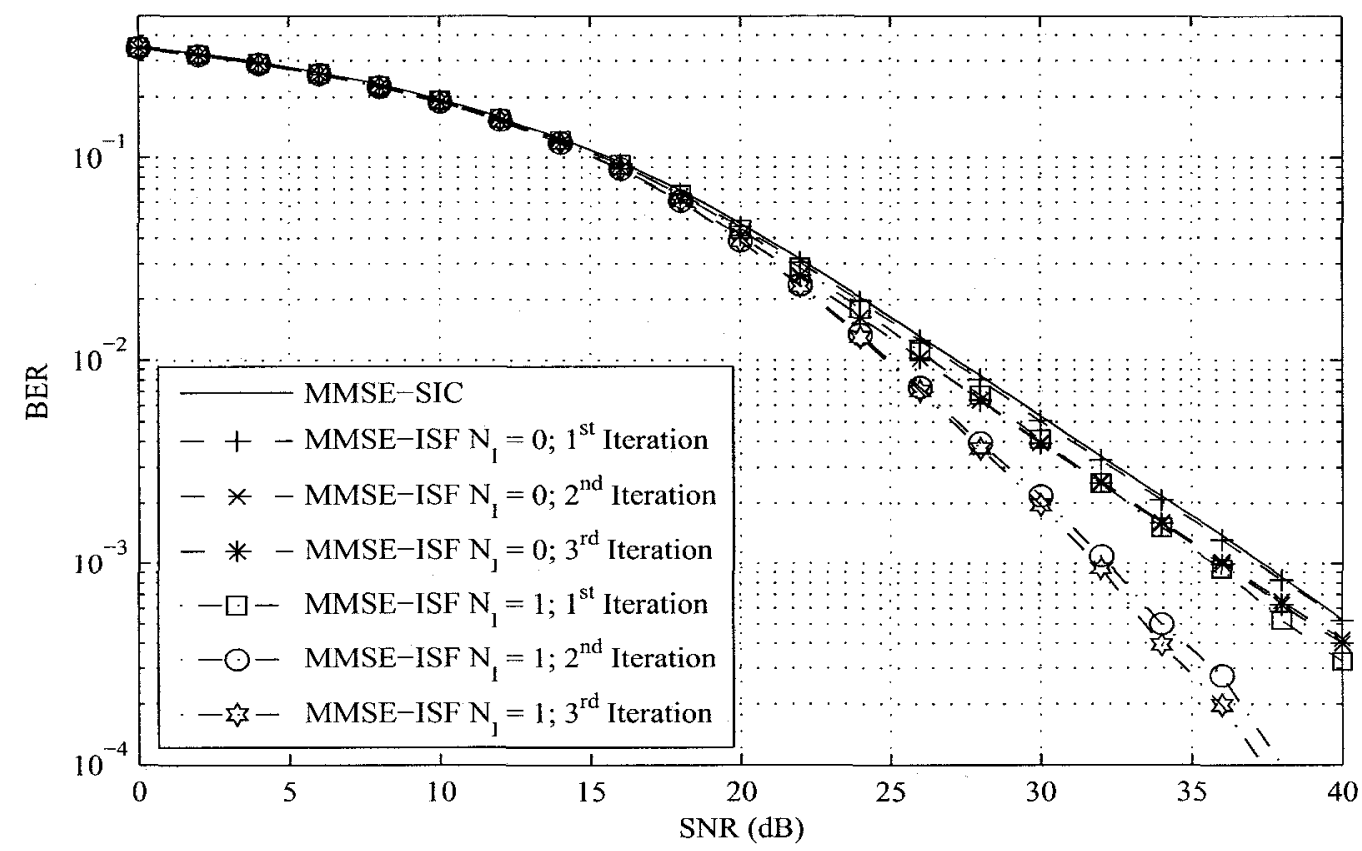

Figure 6.5: BER performance of an uncoded $2 \times 2$ MIMO system using MMSE detectors and 64-QAM.

over MMSE-SIC is $0.7 \mathrm{~dB}$ in the first iteration. By the third iteration the gain over the first is $3.1 \mathrm{~dB}$ at a BER $10^{-3}$. Additionally, when the number of interferers increase to $N_{I}=1$ and $N_{I}=2$ the SNR gain at a BER of $10^{-3}$ is $7.2 \mathrm{~dB}$ and 8.8 $\mathrm{dB}$ respectively. Again, increasing antenna array size increases the gain seen by the MMSE-ISF over the MMSE-SIC detector. The performance of MMSE-ISF using T-SS/LLRE does improve with a larger $N_{I}$, but the gain from $N_{I}=1$ to $N_{I}=2$ is much smaller than that seen from $N_{I}=0$ to $N_{I}=1$, pointing to a diminishing return as $N_{I}$ grows larger. The slope of the BER curves increase as $N_{I}$ increases. This indicates a diversity gain for each additionally considered interferer. That is, we conjecture that the diversity of MMSE-ISF with T-SS/LLRE is $N_{I}+1$.

Figure 6.5 gives the BER performance for the $2 \times 2$ MIMO system when a 64-QAM constellation is transmitted on all subchannels. Aside from the typical 
degrade in performance seen when larger constellations are employed we see that in the first iteration for $N_{I}=0$, MMSE-ISF T-SS/LLRE has at best a $0.25 \mathrm{~dB}$ SNR gain over the MMSE-SIC detector. The SNR gain at $10^{-3}$ of going from the first iteration to the third is $1.3 \mathrm{~dB}$, which is $0.3 \mathrm{~dB}$ less than the case where 16-QAM is used. When $N_{I}=1$ the SNR gain on the third iteration over the first iteration for $N_{I}=0$ at a BER of $10^{-3}$ is $4.25 \mathrm{~dB}$, which is less than the $5 \mathrm{~dB}$ gain when the MIMO system transmits a 16-QAM constellation. These results suggest that the gains made by the MMSE-ISF T-SS/LLRE are smaller for larger constellations. This is explained by the higher probability of error at each layer because a larger constellation has been used. This figure also shows the diversity gain that the $N_{I}=1 \mathrm{~T}$-SS/LLRE has over the $N_{I}=0 \mathrm{~T}$-SS/LLRE as the curve for the first iteration of the $N_{I}=1 \mathrm{~T}$-SS/LLRE crosses the curve for the $N_{I}=0 \mathrm{~T}$-SS/LLRE.

Figure 6.6 is included to give the reader an appreciation of how much T-SS/LLRE is able to reduce the computational complexity required for detection at higher SNR, while still maintaining good BER performance. In order to obtain a BER of $10^{-3}$ MMSE-ISF T-SS/LLRE with $N_{I}=1$ requires an SNR of $31.75 \mathrm{~dB}$. At $31.75 \mathrm{~dB}$ MMSE-ISF T-SS/LLRE requires a total average of about 110 computations - only 14 more than that required by the MMSE-ISF T-SS/LLRE with $N_{I}=0$ - which is much less than the 1024 required by the ML detector. Indeed, for higher SNRs T-SS/LLRE provides good performance for a low average computational cost.

Figures 6.7 and 6.8 give MMSE-ISF T-SS/LLRE BER performance for a $3 \times 3$ and $4 \times 4$ MIMO transceiver transmitting 64-QAM from each transmit antenna. The figures show have the same behaviour as was described for Figures 6.3 and 6.4 with respect to the diversity gains of MMSE-ISF T-SS/LLRE and the SNR gain over MMSE-SIC increasing for larger antenna arrays. 


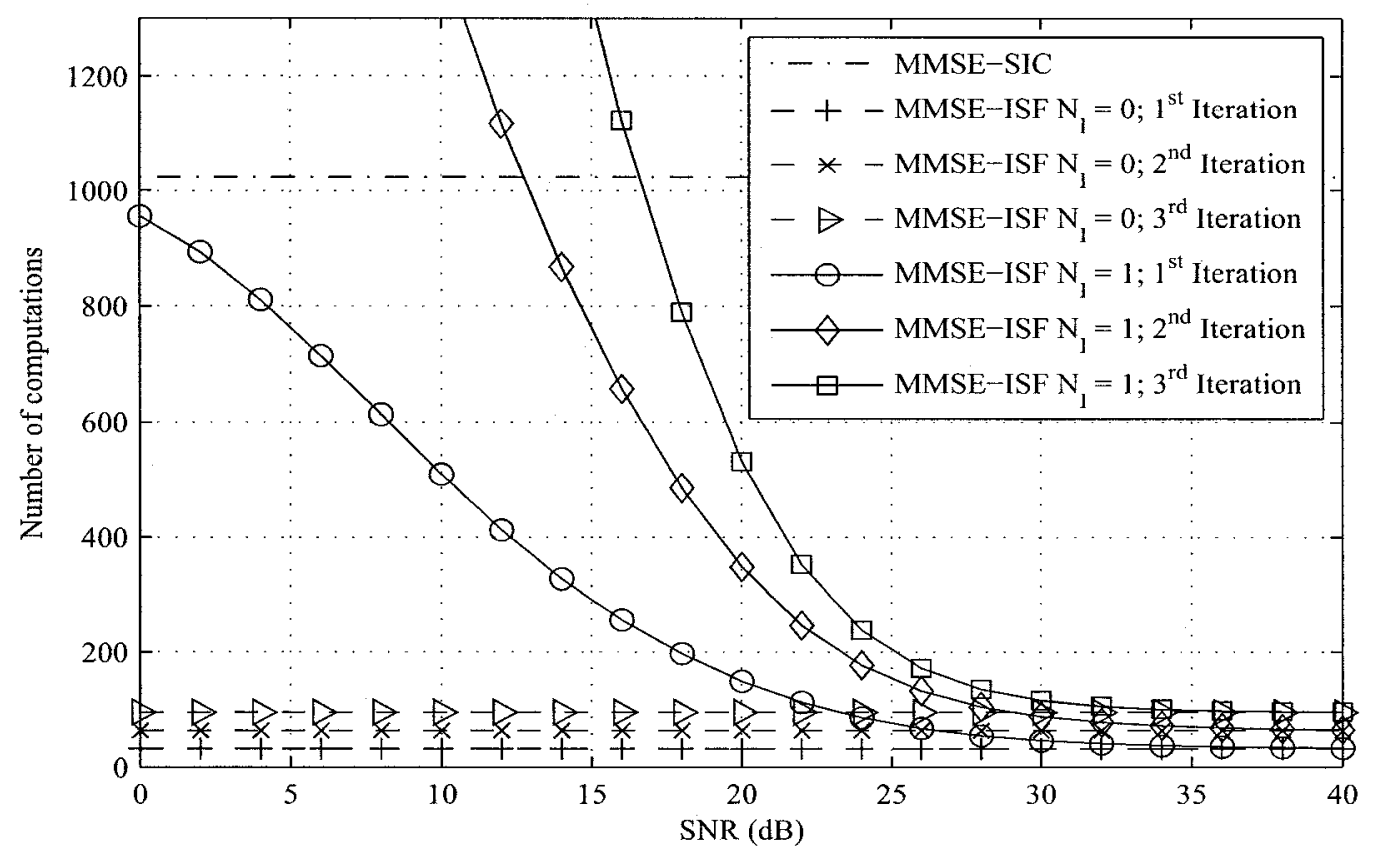

Figure 6.6: Number of exponential computations performed by the $2 \times 2$ MIMO system using the T-SS/LLRE and MMSE-ISF detector and 64-QAM for $N_{I}=1$.

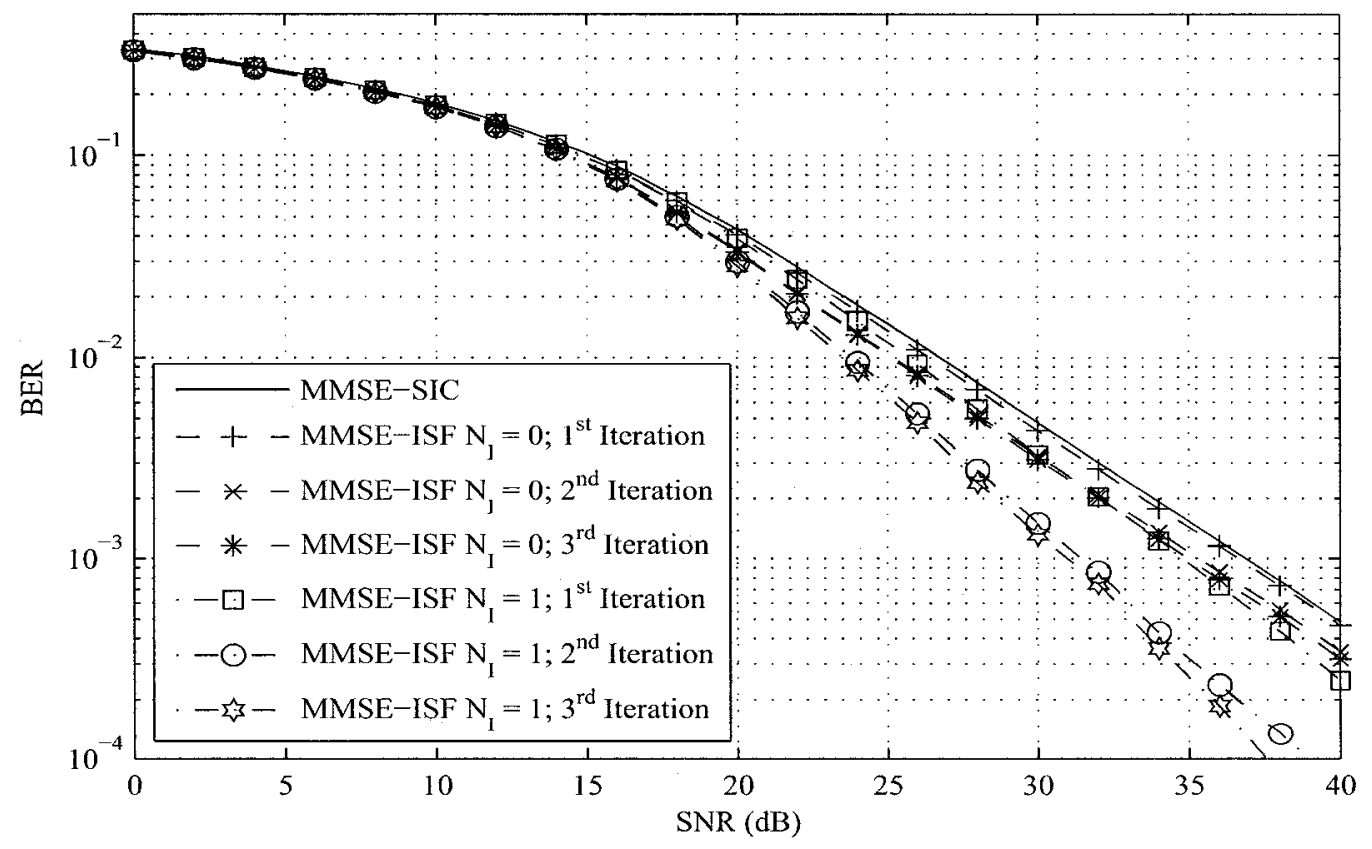

Figure 6.7: BER performance of an uncoded $3 \times 3$ MIMO system using MMSE detectors and 64-QAM. 


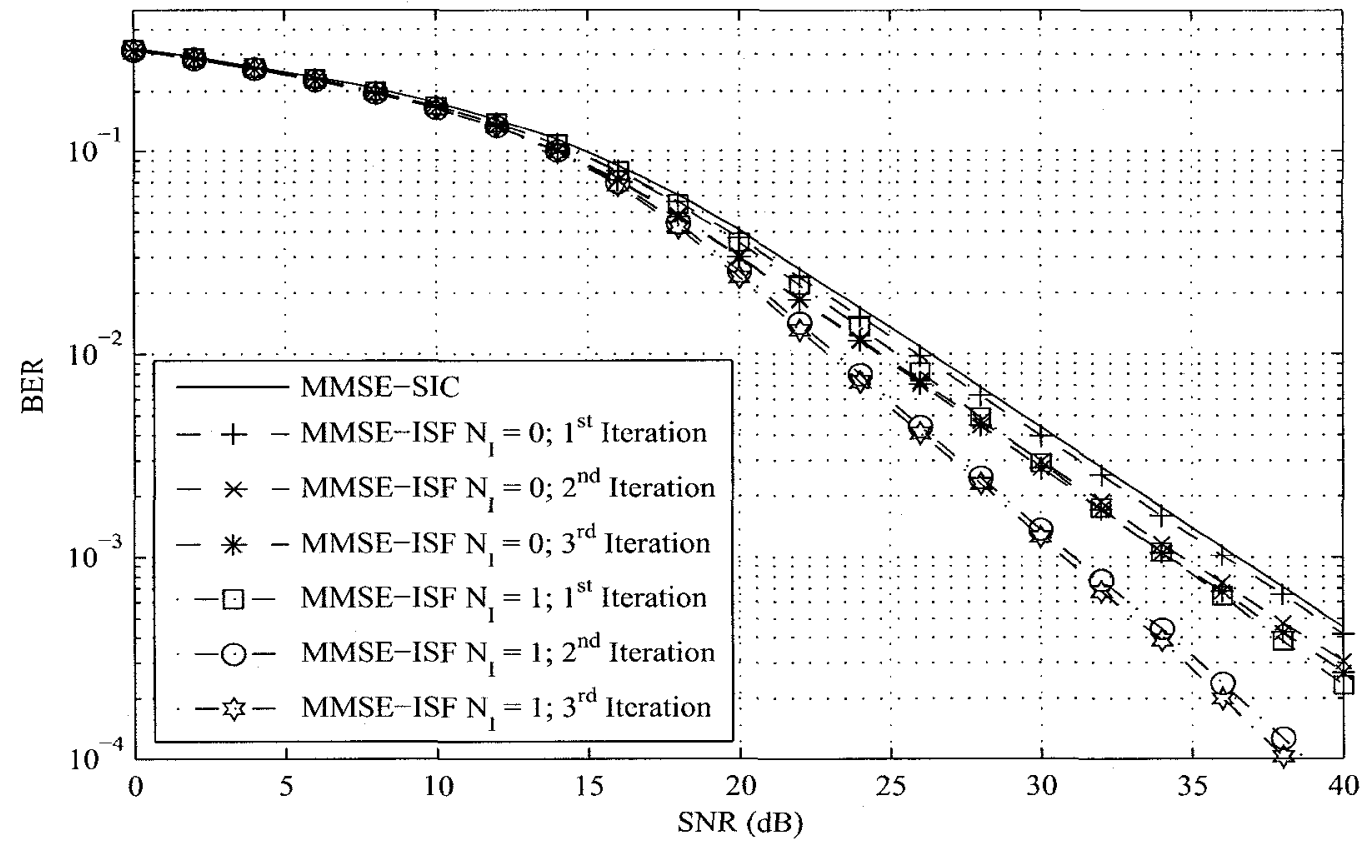

Figure 6.8: BER performance of an uncoded $4 \times 4$ MIMO system using MMSE detectors and 64-QAM.

These results demonstrate that not only is the MMSE-ISF with a T-SS/LLRE able to bring significant SNR gains to the MIMO system, but that the T-SS/LLRE is an excellent addition for improving BER by adding diversity to the linear MIMO detector at a low average computational cost for mid-range to high SNRs.

\subsection{Experimental Throughput Performance of RC/QC-MIMO with T-SS/LLRE}

In this section we give simulation results that demonstrate the throughput performance of RC/QC-MIMO transceivers with a T-SS/LLRE. For all transceivers, bit-rates are allocated using CWFA; the MMSE-ISF detector uses T-SS/LLRE with $N_{I}=1$ (except SVD and SVD-CWFA as they have no MAI) and $P r^{(t h)}=10^{-10}$; 


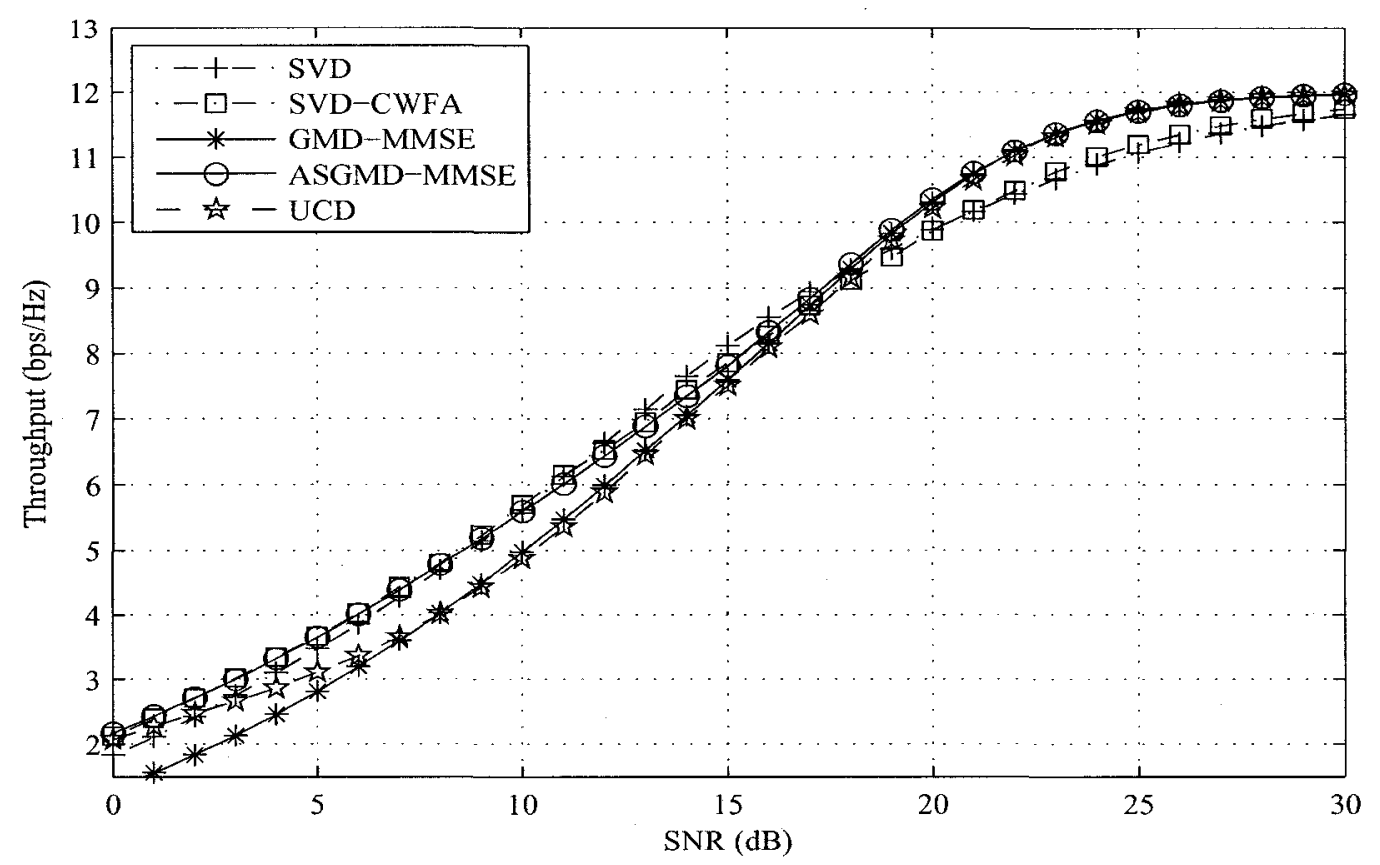

Figure 6.9: Simulated throughput performance of $2 \times 2$ IT MIMO systems when coupled with RC/QC LDPC codes.

and $L_{z}=3$.

Figure 6.9 gives the performance of a $2 \times 2 \mathrm{RC} / \mathrm{QC}-\mathrm{MIMO}$ system with $\mathrm{T}$ SS/LLRE $N_{I}=1$. We see that the same behaviour that was observed for each MIMO transceiver in Chapter 5. For the transceivers of Chapter 5 all MAI was considered Gaussian, corresponding to a T-SS/LLRE with $N_{I}=0$. The difference between the results presented in this chapter and those in Chapter 5 is an improvement in the performance of the RC/QC-MIMO transceivers that suffer from MAI. The SVD-based RC/QC-MIMO systems will not see any gains because they do not experience MAI. For example, the RC/QC-ASGMD using T-SS/LLRE $N_{I}=0$ has a spectral efficiency of $3.65 \mathrm{bps} / \mathrm{Hz}, 7.75 \mathrm{bps} / \mathrm{Hz}$ and $10.26 \mathrm{bps} / \mathrm{Hz}$ at SNRs of $5 \mathrm{~dB}$, $15 \mathrm{~dB}$ and $20 \mathrm{~dB}$ respectively. By contrast the RC/QC-ASGMD using T-SS/LLRE $N_{I}=1$ has a spectral efficiency of $3.66 \mathrm{bps} / \mathrm{Hz}, 7.81 \mathrm{bps} / \mathrm{Hz}$ and $10.36 \mathrm{bps} / \mathrm{Hz}$ for 


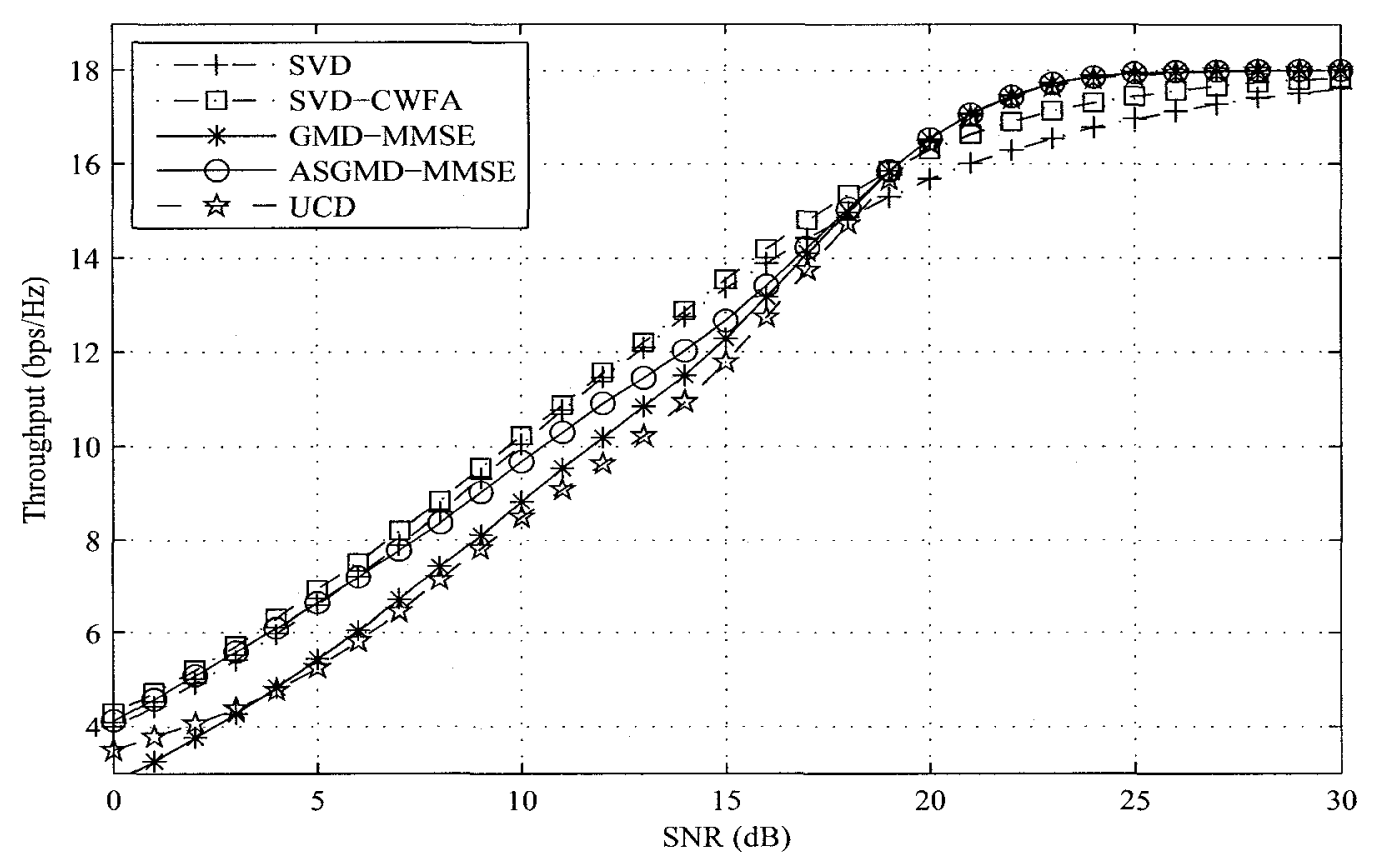

Figure 6.10: Simulated throughput performance of $3 \times 3$ IT MIMO systems when coupled with RC/QC LDPC codes.

those same SNRs. As another example, the RC/QC-GMD using T-SS/LLRE with $N_{I}=0$ has a spectral efficiency $2.79 \mathrm{bps} / \mathrm{Hz}, 7.5 \mathrm{bps} / \mathrm{Hz}$ and $10.21 \mathrm{bps} / \mathrm{Hz}$ at those same SNRs compared with $2.81 \mathrm{bps} / \mathrm{Hz}, 7.6 \mathrm{bps} / \mathrm{Hz}$ and $10.32 \mathrm{bps} / \mathrm{Hz}$ for $N_{I}=1$.

The results show that there is more gain at higher SNRs where the spectral efficiency is higher and these systems have more difficulty dealing with MAI. These results also show that there is not much to be gained for the IT RC/QCGMD-based systems. This is likely due to the fact that GMD-based transceivers already achieve receive diversity and as result no new diversity is gained with the T-SS/LLRE. This, however does not mean that a PIT RC/QC-GMD-based system - which experiences more MAI - would not benefit from the use of a T-SS/LLRE.

Figures 6.10-6.11 give the throughput performances of the $3 \times 3$ and $4 \times 4$ LDPC- 


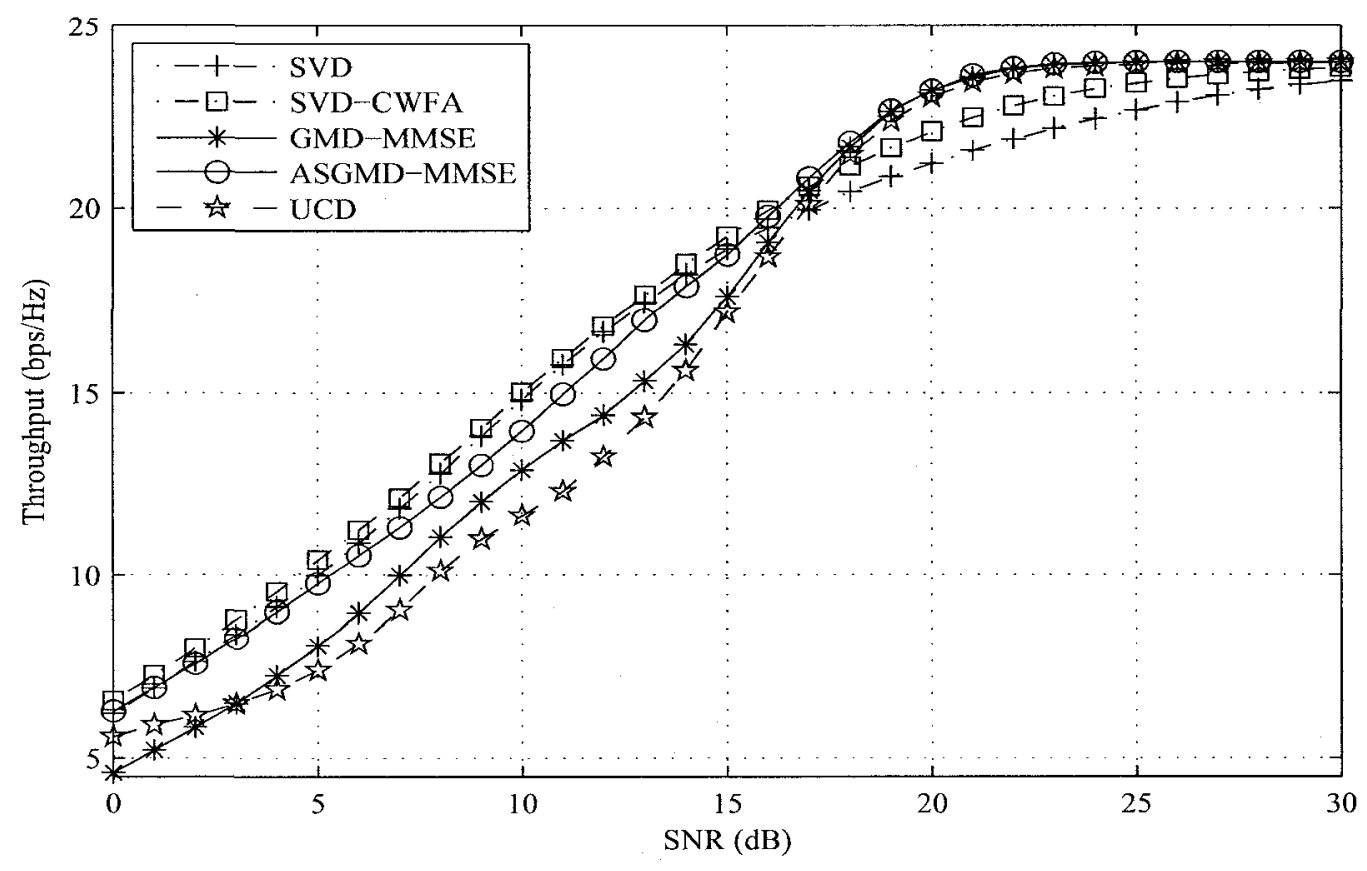

Figure 6.11: Simulated throughput performance of $4 \times 4$ IT MIMO systems when coupled with RC/QC LDPC codes.

MIMO systems. In these figures we view the same behaviour as in Figure 6.9. That is, the throughput performance of RC/QC-MIMO improves for the T-SS/LLRE with $N_{I}=1$.

With better ability to resolve and estimate MAI, the RC/QC-MIMO systems that experience MAI and are implemented using T-SS/LLRE with $N_{I}>0$ have better throughput performance than those that assume the MAI is Gaussian. 


\section{Chapter 7}

\section{Compromising Between SVD and GMD: A Hybrid Transceiver}

In this chapter a new Hybrid transceiver comprised of the SVD and ASGMD transceivers is introduced and evaluated. This Hybrid MIMO system selects between the SVD and GMD transceiver and chooses a subset of subchannels to transmit on.

\subsection{Hybrid Transceiver Design}

We have seen in Chapters 4 - 6 that the GMD transceiver is throughput-limited at low- to mid-range SNRs by its inability to adequately cancel the MAI in the upper triangular portion of its equivalent channel matrix. Also demonstrated was the slow convergence of the SVD-based transceivers to the system's maximum nominal rate. The ASGMD transceiver was shown capable of delivering the low SNR performance that the GMD transceiver could not; however, it suffered from the same difficulties as the GMD transceiver did for mid-range SNRs where the ASGMD begins to use more subchannels. This gives ASGMD the same MAI resolvability issue that the GMD suffers from for mid-range SNRs. Indeed, it would be vory useful to have a MIMO transceiver that combined the low- and high-SNR performance of ASGMD 
with the mid-range SNR performance of SVD. This wish inspired a simple solution: create a Hybrid SVD/ASGMD transceiver.

The Hybrid transceiver operates by choosing between the GMD and SVD transceivers, and a number of subchannels, $L$, on which to transmit. This requires the creation of a new transceiver that does antenna selection based on the SVD transceiver, which we call ASSVD. The ASSVD uses (5.2) to do CCAS. The receiver is tasked with selecting the transceiver, ASSVD or ASGMD, which gives the best throughput for the Hybrid RC/QC-MIMO transceiver. Intuitively, a good solution may be to select the transceiver that gives the best instantaneous constrained capacity using (4.16); however in Section 4.2.3 we saw that in theory ASGMD will always have better theoretical constrained capacity than SVD-CWFA, due to its assumptions that there is no error propagation in layered detection. Since ASSVD does discrete power allocation it will often perform worse than SVD-CWFA, and ASGMD, as a result. However, this was disproven by the experimental results of the last three chapters. This means that we cannot rely on the total constrained rate determined using (4.16) to select the appropriate transceiver.

The optimal solution would be to run an infinite number of simulations for each transceiver for the current channel realization to find out which one has the highest throughput. Of course, this solution is not tractable and cannot be feasibly implemented. If we cannot choose the proper transceiver using the simulated performance of ASSVD and ASGMD for an instantaneous channel realization, we can still do so for the average case. That is, we choose the proper transceiver and number of subchannels using the simulated average throughput performance of each transceiver. For a given pre-filtering SNR we wish to choose the transceiver with the largest average 


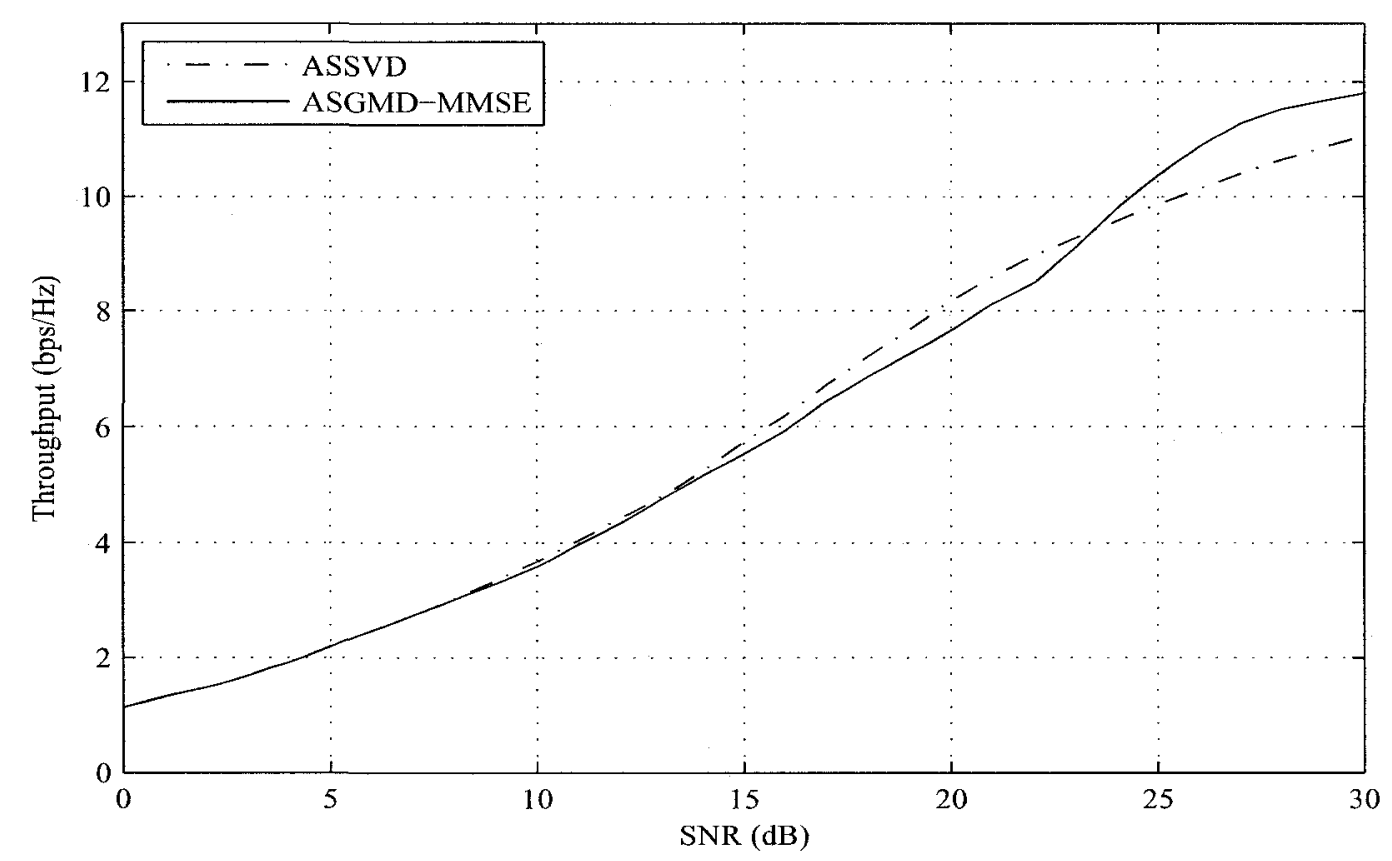

Figure 7.1: Simulated throughput performance of $2 \times 2$ RC/QC-ASSVD and -ASGMD for a normalized channel.

throughput. To obtain the pre-filtering SNR for a given channel realization we use

$$
\mathrm{SNR}=\frac{E_{s}}{N_{0}} \operatorname{Tr}\left\{\mathbf{H H}^{+}\right\}
$$

In order to run simulations for the throughput of each transceiver realization at a given fixed SNR, we generate normalized realizations of the channel matrix

$$
\mathbf{H}^{\prime}=\frac{\mathbf{H}}{\sqrt{\operatorname{Tr}\left\{\mathbf{H H}^{+}\right\}}}
$$

where $\mathbf{H}^{\prime}$ is a normalized channel matrix realization. Now the channel model of Chapter 2 uses $\mathbf{H}^{\prime}$ instead of $\mathbf{H}$ as its channel matrix. That is, (2.2) becomes

$$
\mathbf{r}=\mathbf{H}^{\prime} \mathbf{x}+\mathbf{n}
$$

Figure 7.1 shows the average throughput performance of the $2 \times 2$ RQ/QC-ASSVD and -ASGMD systems for a normalized channel matrix. The T-SS/LLRE $N_{I}=1$ is used by RC/QC-ASGMD, both transceivers employ adaptive modulation and use 
$L_{z}=3$. We see that each transceiver realization has a SNR region were it dominates. That is, ASSVD transceiver has the highest throughput performance in the SNR range $[-\infty, 23.22] \mathrm{dB}$, and the ASGMD has better performance outside that range. Using this information a lookup table can be made using the following selection rule

$$
\mathrm{H}_{L}(\gamma)= \begin{cases}\text { ASSVD } & \text { if } \gamma<23.42 \mathrm{~dB} \\ \text { ASGMD } & \text { otherwise }\end{cases}
$$

where $\mathrm{H}_{L}(\gamma)$ is a look-up table that chooses the appropriate transceiver for the $2 \times 2$ Hybrid transceiver for a SNR $\gamma$. Hybrid transceivers for larger antenna arrays can be generated using this method of generating look-up tables based on the average throughput performance of ASSVD and ASGMD in a normalized channel.

The Hybrid transceiver is simple to implement due to the similarities between the SVD and GMD transceivers. The GMD transceiver uses the SVD matrix decomposition to compute its matrix decomposition, so the computation of the SVD and GMD can be done in tandem. Both transceivers require the same type of feedback information: A semi-unitary matrix and a set of bit-rate allocations for each subchannel. This means that the receiver can feedback the CSI required for the Hybrid transceiver without needing to inform the transmitter which transceiver was selected. This means that the Hybrid transceiver can be implemented with no additional complexity.

\subsection{Experimental Throughput Performance of the Hybrid Transceiver}

This section demonstrates the experimental throughput performance of the $\mathrm{RC} / \mathrm{QC}$ Hybrid transceiver described in the previous section. For all experimental results, the RC/QC-ASGMD and -Hybrid transceivers use the T-SS/LLRE with $N_{I}=1$; 


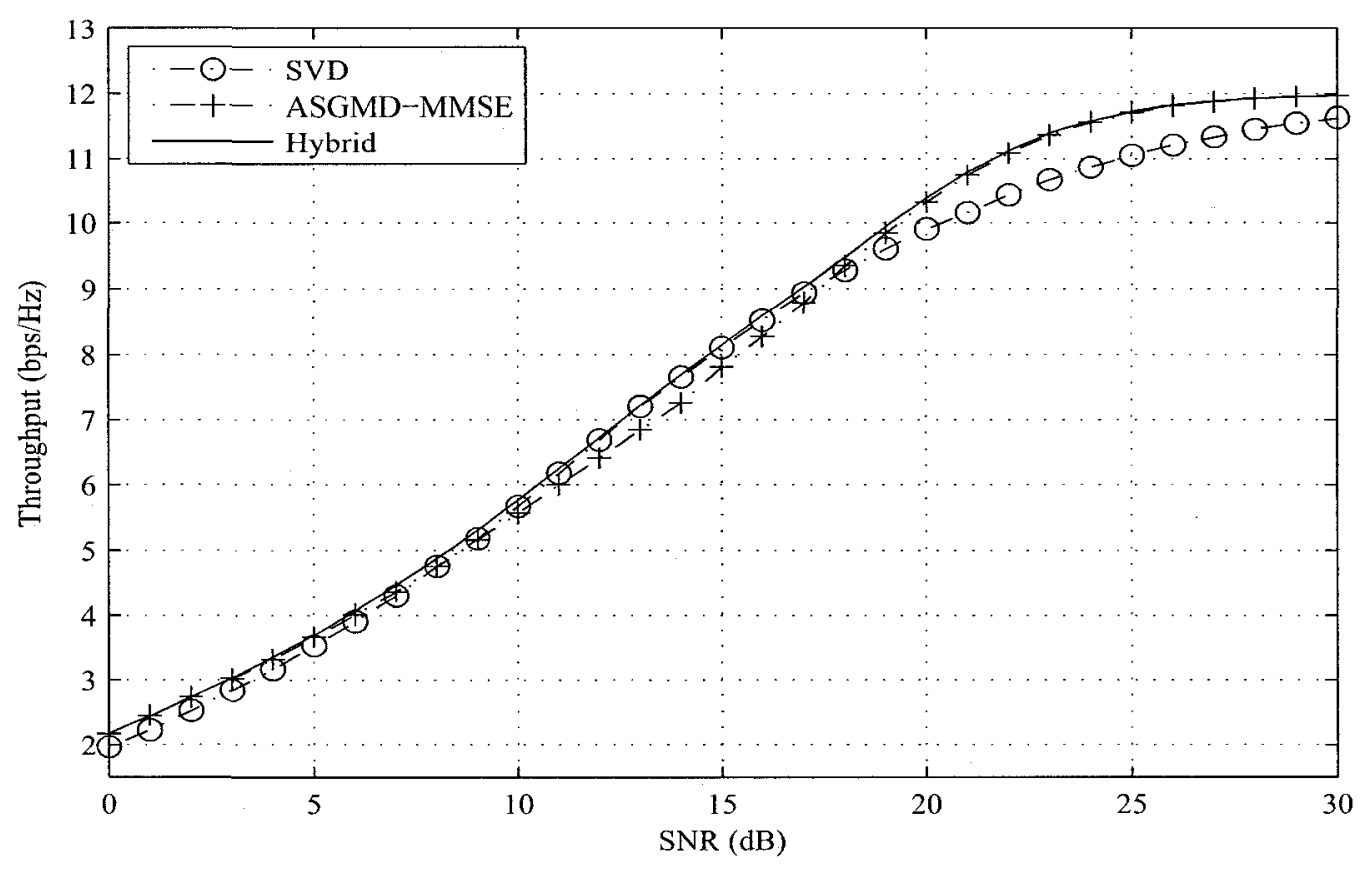

Figure 7.2: Experimental throughput performance of $2 \times 2$ IT Hybrid transceiver when coupled with RC/QC LDPC codes.

adaptive modulation based on the CWFA is used; and $L_{z}=3$.

Figure 7.2 gives the performance of the $2 \times 2$ RC/QC-Hybrid system when compared with its parent transceivers (SVD and ASGMD). The RC/QC-Hybrid system delivers throughput performance that is either equal to or better than that given by RC/QC-ASGMD and RC/QC-SVD.

Figures 7.3 and 7.4 demonstrate the throughput performance of the $3 \times 3$ and $4 \times 4 \mathrm{RC} / \mathrm{QC}-H y b r i d$ systems respectively. The throughput performance is similar to that described of Figure 7.2, except that the throughput performance gain at lower SNRs of RC/QC-Hybrid over RC/QC-ASGMD become greater as the size 


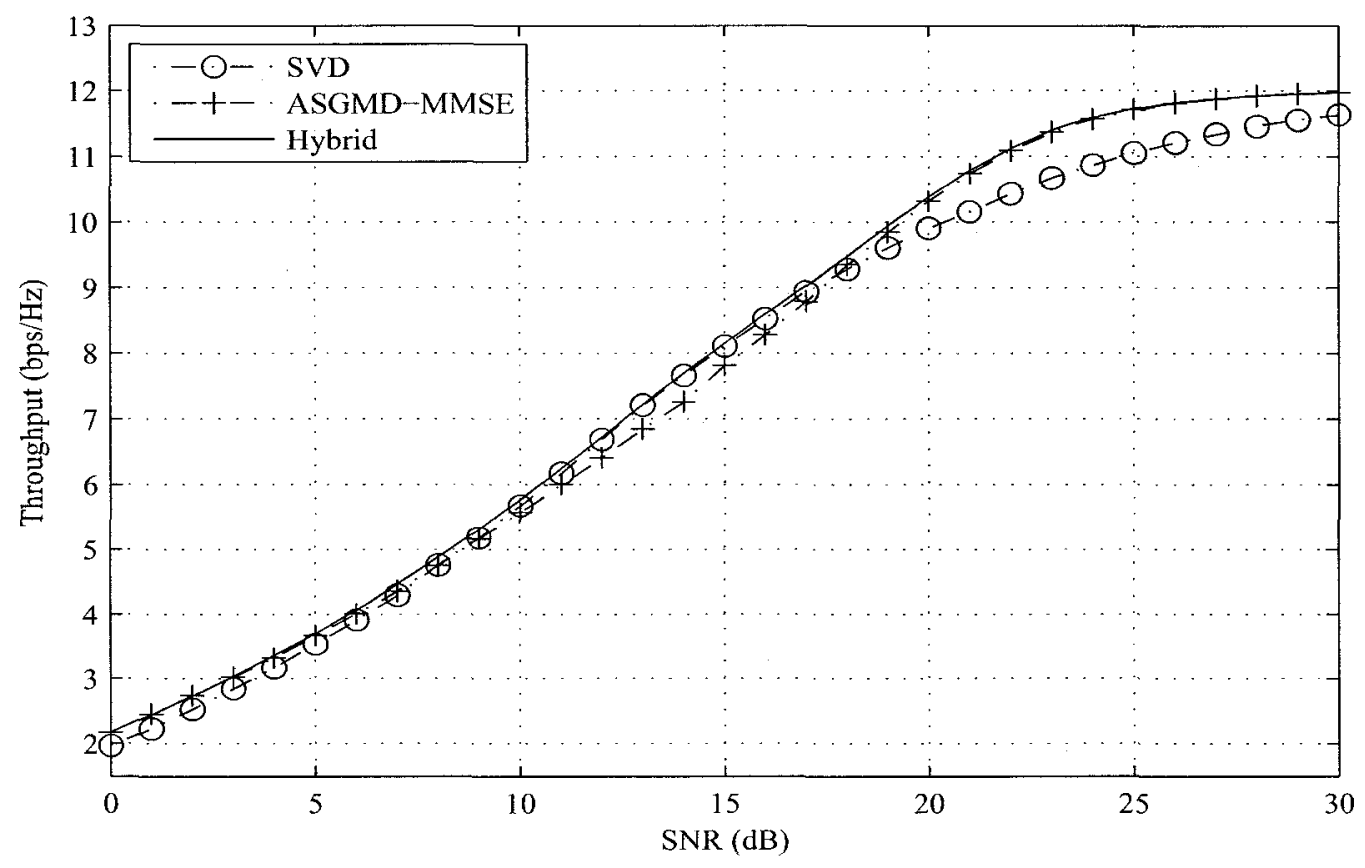

Figure 7.3: Experimental throughput performance of $3 \times 3$ IT Hybrid transceiver when coupled with RC/QC LDPC codes.

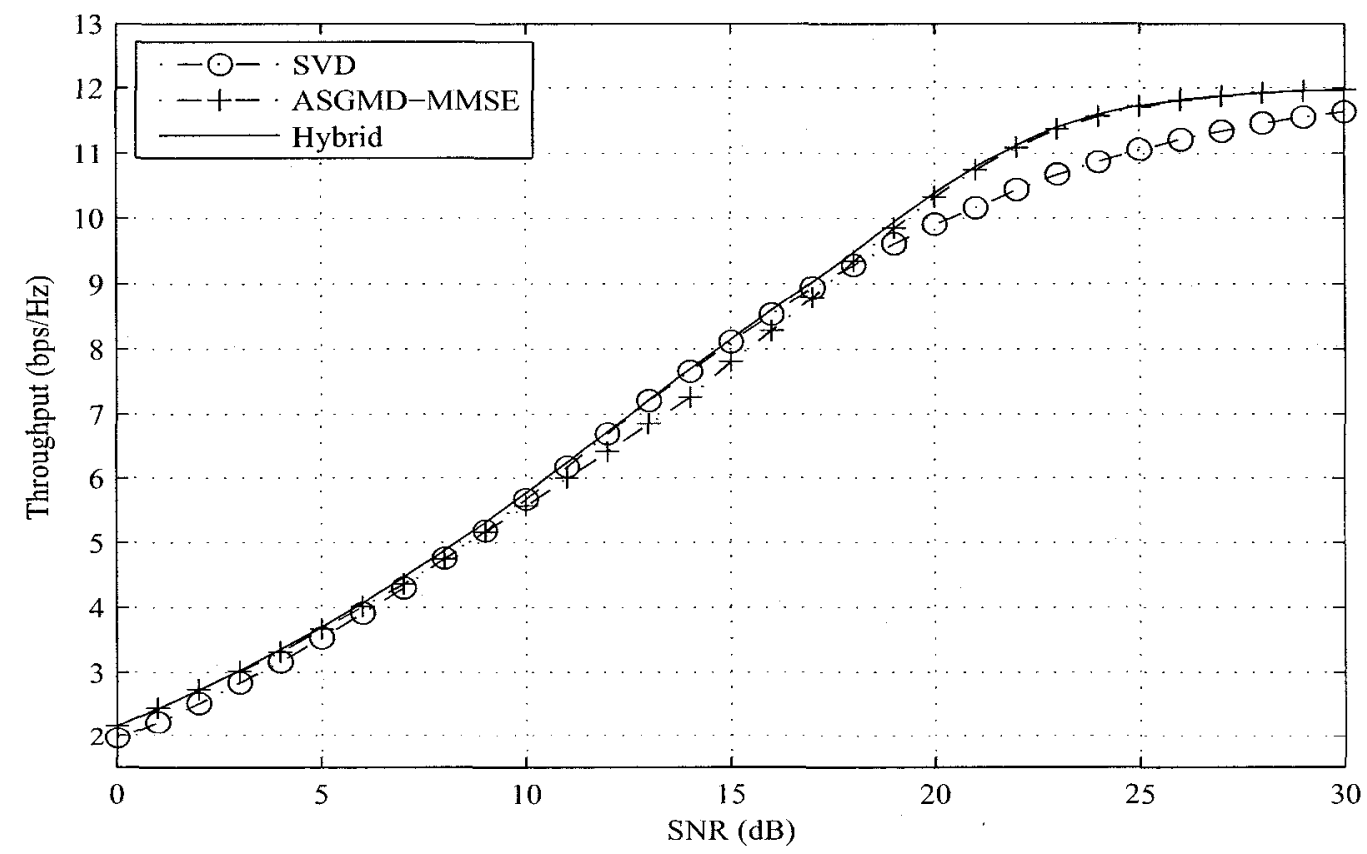

Figure 7.4: Experimental throughput performance of $4 \times 4$ IT Hybrid transceiver when coupled with RC/QC LDPC codes. 
of the antenna array is increased. These results show that the simple RC/QCHybrid transceiver is capable of delivering the low/high SNR performance of RC/QCASGMD, while achieving the mid-range SNR performance of RC/QC-SVD. 


\section{Part III}

\section{MIMO Transceiver Design for}

\section{Systems with Partial CSI Feedback}




\section{Chapter 8}

\section{Practical CSI feedback for the Partially Informed Transmitter}

There are some cases, such as TDD wireless systems, where the state of the channel is at least partially known to the transmitter, however there are many wireless systems that will require some sort of feedback information in order to have CSI available to the transmitter. So far we have discussed MIMO systems for which all CSI is known to the transmitter and have called this system IT. The IT is more of an academic model which allows us to have a benchmark with which to compare performance. Realistically, a MIMO system which requires CSI to be fed back to the transmitter will have to make due with a limited amount of CSI. This is because it would be impossible to relay the infinite amount of information required to enable a wireless system with perfect channel knowledge at the transmitter. It is more practical to send back a limited amount of channel information and choose from a set of quantized parameters. Systems with partial CSI at the transmitter were first dubbed as PIT in Chapter 3 and will be the main focus of this chapter. 


\subsection{Classification of the Types of Quantizable In- formation and the Amount of Feedback Re- quired by each MIMO Transceiver}

In relaying a limited amount of CSI back to the transmitter a set of parameters must be chosen by which to represent the information that is pertinent to the transmitter. Intuitively, it may seem simple to relay the channel magnitude and phase parameters $\left(\left|h_{i j}\right|\right.$ and $\left.\angle h_{i j}\right)$ back to the transmitter to reconstruct the channel; from there the transmitter could compute the precoding parameters. However, this method would be difficult to implement since the magnitude parameters vary over a large range $([0, \infty])$. It is better to find parameters that can be quantized over a shorter interval as this will reduce the quantization error. Since the precoders of the transceivers described in this thesis are composed of semi-unitary and diagonal matrices it is simpler to quantize the parameters of each matrix independently. In our study there are three types of quantizable information: semi-unitary precoder matrices, diagonal power allocation matrices and the bit-rate used in adaptive modulation. In this section we will investigate the cost and difficulties that come with quantizing each type of information with respect to each MIMO transceiver described in Section 2.3 and the one proposed in Chapter 7 .

\subsubsection{Feedback Requirements of SVD-CWFA}

The precoder matrix, F, of SVD-CWFA is composed of a $N_{t} \times N_{t}$ complex semiunitary matrix, $\boldsymbol{\Omega}_{1}$, and a $N_{t} \times L$ diagonal power allocation matrix. That is, $\mathbf{F}=\mathbf{V} \boldsymbol{\Phi}$. In addition to this, SVD-CWFA uses adaptive modulation. With each subchannel possibly having a different SINR, they will require separate bit-rates. The bit-rate allocated to each subchannel reveals some information about the semi-unitary and 
power allocation matrix of SVD-CWFA. Any zero bit-rates also mean a zero-power allocation. A zero-power allocation for subchannel $i$ means that there is no need to send back information about this power. That is, $\Phi$ is represented by the feedback of $L$ power allocations. For the semi-unitary matrix of SVD-CWFA a zero bit-rate for subchannel $i$ means that information about column vector $i$ of $\mathbf{V}$ does not need to be fed back. Therefore, there are $L N_{t} \times 1$ column vectors required to represent $\Omega_{1}$. Information about the semi-unitary matrix, $\Omega_{1}$, power allocation matrix, $\boldsymbol{\Phi}$, and bit-rate allocation for each subchannel of SVD-CWFA must be sent back to the transmitter.

\subsubsection{Feedback Requirements of SVD}

The SVD precoder matrix is composed of a $N_{t} \times N_{t}$ complex semi-unitary matrix, $\Omega_{1}$, and a $N_{t} \times K$ power allocation matrix. That is, $\mathbf{F}=\mathbf{V} \boldsymbol{\Phi}$. Additionally, SVD uses adaptive modulation, with each subchannel possibly requiring a separate bitrate allocation. SVD uses all $K$ active subchannels, which are those that are assigned non-zero bit-rates. This also means that $K$ of the $N_{t}$ column vectors of $\mathbf{V}$ need to be represented at the transmitter. Those column vectors are identified by a non-zero bitrate for their respective subchannels. For SVD, the transmitter requires information about the semi-unitary matrix and individual bit-rates for each subchannel.

\subsubsection{Feedback Requirements of GMD}

The GMD precoder matrix is composed only of a $N_{t} \times N_{t}$ complex semi-unitary matrix, $\Omega_{1}$, and a $N_{t} \times K$ power allocation matrix, $\boldsymbol{\Phi}$. That is, $\mathbf{F}=\mathbf{P} \boldsymbol{\Phi}$. Additionally, GMD uses adaptive modulation, with each subchannel possibly requiring a separate bit-rate, if MMSE filtering is used. Without MMSE nulling vectors, a regular GMDSIC is used, and since equal gain subchannels are assumed the bit-rate is the same 
on all subchannels. We are more interested in GMD-MMSE, since it is shown to have better throughput and BER performance than GMD-SIC, as is demonstrated in Chapter 3. The power on each employed subchannel is assigned using (2.24), where $K$ is obtained from the number subchannels with non-zero bit-rates. Additionally, the GMD requires only the $K$ column vectors from $\mathbf{P}$, which correspond to subchannels with a non-zero bit-rates. So, for GMD, the transmitter requires only information about a semi-unitary matrix and individual bit-rates for each subchannel.

\subsubsection{Feedback Requirements of ASGMD}

The ASGMD transceiver, being based upon the GMD has exactly the same precoder matrix structure (i.e. $\mathbf{F}=\mathbf{P} \Phi$ ) and, as a result, feedback requirements. This is because ASGMD is GMD, but using a subset of $L$ GMD subchannels. As was described in the previous subsection, the number of subchannels used is determined by the number of non-zero bit-rates. So, ASGMD requires the feedback of a $N_{t} \times L$ semi-unitary matrix, and the bit-rate of each subchannel.

\subsubsection{Feedback Requirements of UCD}

The UCD precoder matrix is composed of two complex semi-unitary matrices, $\Omega_{1}$ and $\boldsymbol{\Omega}_{2}$, and a power allocation matrix, $\boldsymbol{\Phi}$. That is, $\mathbf{F}=\mathbf{V} \boldsymbol{\Phi} \boldsymbol{\Omega}_{2}$. The UCD transceiver gives equal SINR subchannels and, as a result, the bit-rate on all subchannels is the same. Since the implementation of the UCD algorithm in Chapter 2 uses $L=N_{t}$, information is required about every parameter. That is, the UCD transmitter requires feedback information about $\mathbf{V}, \boldsymbol{\Omega}_{2}, \boldsymbol{\Phi}$ and the bit-rate, which is the same for all subchannels. 


\subsubsection{Feedback Requirements of Hybrid}

The Hybrid transceiver proposed in Chapter 7 selects between the ASSVD and ASGMD transceivers. The ASSVD and ASGMD precoders are both composed of a semi-unitary matrix, $\Omega_{1}$, and a power allocation matrix, $\boldsymbol{\Phi}$. That is, $\mathbf{F}=\Omega_{1} \boldsymbol{\Phi}$. The bit-rate can be different on each subchannel the number of non-zero bit-rates indicates the number of subchannels, $L$, used. Since ASGMD and ASSVD have the same precoder requirements, the transmitter does not need to be aware of which of ASSVD or ASGMD the Hybrid transceiver has selected. Thus, to create the Hybrid precoder, the transmitter requires information about the semi-unitary matrix, $\boldsymbol{\Omega}_{\mathbf{1}}$, and the bit-rate allocated to each subchannel.

\subsubsection{Comparison of the Feedback Requirements of the Dif- ferent MIMO Transceivers}

In Sections 8.1.1 - 8.1.6 we discussed the feedback requirements of the MIMO transceivers described in Section 2.3 and the Hybrid transceiver proposed in Chapter 7. In this subsection we discuss the amount of feedback that is required for each type of feedback and compare the transceivers using this information.

\section{Semi-unitary Matrix Feedback}

A $N \times M$ complex semi-unitary matrix, $\mathbf{V}$, can be described by $N M$ real magnitude and phase parameters each representing one of the $N M$ entries of $\mathrm{V}$. A simple quantization technique is used where all magnitude and phase parameters of $\mathbf{V}$ are quantized independently and using the same number of feedback bits, $x_{q}$. This simple, but inefficient technique requires a total of $2 x_{q} N M$ to quantize matrix $\mathrm{V}$. 
Table 8.1: Comparison of the instantancous feedback requirements of different MIMO transceivers.

\begin{tabular}{|l||c|c|c|}
\hline Parameter & SVD-CWPA & SVD & GMD \\
\hline \hline Bit-rate Allocation & $N_{t} \log _{2}(5)$ & $N_{t} \log _{2}(5)$ & $N_{t} \log _{2}(5)$ \\
\hline Power Allocation & $x_{p} L$ & N/A & N/A \\
\hline Semi-Unitary Matrix/ces & $2 x_{q} N_{t} L$ & $2 x_{q} N_{t} K$ & $2 x_{q} N_{t} K$ \\
\hline Parameter & ASGMD & UCD & Hybrid \\
\hline Bit-rate Allocation & $N_{t} \log _{2}(5)$ & $\log _{2}(5)$ & $N_{t} \log _{2}(5)$ \\
\hline Power Allocation & $\mathrm{N} / \mathrm{A}$ & $x_{p} N_{t}$ & N/A \\
\hline Semi-Unitary Matrix/ces & $2 x_{q} N_{t} L$ & $2 \times\left(2 x_{q} N_{t}^{2}\right)$ & $2 x_{q} N_{t} L$ \\
\hline
\end{tabular}

\section{Power Allocation Matrix Feedback}

A diagonal $N \times L$ power allocation matrix, $\boldsymbol{\Phi}$, has $L$ non-zero entries to represent. Let us suppose that all entries in the power allocation matrix are quantized independently using $x_{p}$ bits. This means that a total of $x_{p} L$ bits are required to quantize $\Phi$.

\section{Bit-Rate Allocation Feedback}

When adaptive modulation is used, each subchannel is assigned a constellation size. In this thesis, the set of constellations, $\mathcal{B}$, used for adaptive modulation has five choices (if we include a size-0 QAM constellation as an option). This means that $\log _{2}(5)$ bits are needed to select a bit-rate for each subchannel. If there are $N$ subchannels, each requiring a different bit-rate, the total number of bits required to do bit-rate allocation is $N \log _{2}(5)$ bits. For the case were all subchannels are assigned the same bit-rate, the number of feedback bits required to do bit-rate allocation is $\log _{2}(5)$ bits.

Table 8.1 lists the instantaneous feedback requirements of each MIMO transceiver based on the description of their precoders, given in Sections 8.1.1 - 8.1.6. From 
the simplistic quantization strategies described in this subsection, it appears that the most expensive component of the precoder to feedback is a semi-unitary matrix. The semi-unitary requires at most $x_{q} N_{t}^{2}$ feedback bits to represent it (i.e. when $L=N_{t}$ ). This means that for the quantization technique described in this subsection the number of bits used to quantize a semi-unitary matrix has quadratic growth with respect to $N_{t}$. However, a semi-unitary matrix is required by all transceivers and, as a result, is a necessity. The next most expensive parameter depends upon which of $\log _{2}(5)$ and $x_{q}$ is greater. Although, both the power allocation matrix and subchannel bit-rates have linear growth with respect to $N_{t}$, except for UCD where the number of feedback bits required to assign bit-rates is constant.

Table 8.1 reveals that the SVD, GMD, ASGMD and Hybrid transceivers all have very similar instantaneous feedback requirements. All four need to feedback a semi-unitary matrix, and a bit-rate for each subchannel. They do not require a power allocation matrix, which is required by the SVD-CWFA and UCD transceivers. The bit-rate allocation of all transceivers has linear growth with the number of subchannels, except for the UCD transceiver. This means that for bit-rate allocation, the UCD transceiver has an advantage over all other transceivers. However, the UCD transceiver has two semi-unitary matrices to quantize, which negates the bit-rate allocation advantage since quantizing an additional semi-unitary matrix will require more bits of feedback that an additional $N_{t}-1$ bit-rate allocations. On average, because of antenna selection, the SVD-CWFA, ASGMD and Hybrid transceivers require the least amount of semi-unitary matrix feedback, since at lower SNRs they will use less subchannels. However, the SVD-CWFA also requires the feedback of a power allocation matrix, making it a less attractive option. Overall, in terms of limiting the number of bits of feedback required it appears that ASGMD and Hybrid are our best options. 
In the next sections we study PIT MIMO systems that used use adaptive modulation and require the feedback of a semi-unitary matrix, but not of a power allocation matrix. So we drop the SVD-CWFA and UCD transceivers from all future discussions in this chapter.

\subsection{The Impact of Quantization Errors and Detec- tor Design for a PIT MIMO System}

Given that the semi-unitary precoder matrix - referred to as a precoder matrix from this point on, for brevity - is constructed using a limited amount of precoder feedback information, there will be quantization errors in the equivalent matrix. Our simulation results demonstrate that left unchecked, these quantization errors can severely limit the performance of the PIT MIMO system. This section demonstrates the impact that quantization errors have on the MAI cancelling capabilities of a detector using SIC and also proposes MMSE nulling filters that are matched to the equivalent channel matrix, which contains quantization errors.

\subsubsection{An Example of the Impact of Quantization Errors: PIT GMD-SIC}

The GMD-SIC transceiver was designed assuming that perfect CSI is available at the transmitter. The receiver creates an ideal equivalent channel matrix, $\mathbf{G}_{I T}$, which it uses to do SIC detection. However, when using a PIT the equivalent channel matrix, $\mathbf{G}_{P I T}$, is not the same. That is

$$
\mathbf{G}_{P I T} \neq \mathbf{G}_{I T}
$$


The difference between the two matrices is due to the quantization error that occurs when using partial CSI to quantize a precoder matrix. When we quantize the precoder matrices, quantization errors can either increase or - more often - decrease the SINR of a subchannel significantly, due to additional MAI. For illustration we use the GMD algorithm with PIT. Suppose the quantized precoder matrix $\mathbf{F}_{P I T}=\mathbf{P}+\Delta$ is used instead of the appropriate precoder matrix $\mathbf{F}_{I T}=\mathbf{P}$; where $\Delta$ is the quantization error matrix. The equivalent channel matrix is

$$
\begin{aligned}
\mathbf{G}_{P I T} & =\mathbf{W H F}_{P I T} \\
& =\mathbf{Q}^{+} \mathbf{H}(\mathbf{P}+\mathbf{\Delta}) \\
& =\mathbf{R}+\mathbf{Z} \\
& =\mathbf{G}_{I T}+\mathbf{Z}
\end{aligned}
$$

where $\mathrm{Z}$ is the resulting error matrix. When using the GMD-SIC we expect the equivalent matrix to be upper triangular. This is not so with the quantization errors added by the error matrix, $\mathbf{Z}$. This means that the lower triangular portion of $\mathbf{G}_{P I T}$ is not zero and the first layer to be detected will see MAI, which is not experienced when using a GMD-SIC with an IT. The $i^{\text {th }}$ decision variable for GMD-SIC is created from the spatially filtered received samples and is expressed as

$$
\begin{aligned}
z_{i} & =y_{i}-\sum_{j=1, j \neq i}^{L} g_{i, j}^{I T} \hat{s}_{j}+\nu_{i} \\
& =g_{i, i}^{P I T} s_{i}+\sum_{j=1, j \neq i}^{L}\left(g_{i, j}^{P I T} s_{j}-g_{i, j}^{I T} \hat{s}_{j}\right)+\nu_{i}
\end{aligned}
$$

where $\left\{g_{i, j}^{I T}\right\}$ is an entry in $\mathbf{G}_{I T} ;$ and $\left\{g_{i, j}^{P I T}\right\}$ is an entry in $\mathbf{G}_{P I T}$. If we assume that the symbol estimation is perfect (i.e. $\hat{s}_{j}=s_{j}$ ) and noise is negligible due to a high 
SNR, the decision variable of (8.2) becomes

$$
\begin{aligned}
z_{i} & =g_{i, i}^{P I T} s_{i}+\sum_{j=1, j \neq i}^{L}\left(g_{i, j}^{P I T}-g_{i, j}^{I T}\right) s_{j} \\
& =g_{i, i}^{P I T} s_{i}+\sum_{j=1, j \neq i}^{L} z_{i, j} s_{j}
\end{aligned}
$$

where $\left\{z_{i, j}\right\}$ is an entry in $\mathbf{Z}$. This means that no matter how high the SNR of the received signals, there will be an error floor if we do not account for the quantization error created at the precoder. A better SIC will match to the equivalent channel $\mathbf{G}_{P I T}$ to do MAI cancelling.

\subsubsection{Equivalent Channel Matrix-Matched MMSE-SIC and MMSE-ISF Filters for PIT MIMO Transceivers}

As was discussed in the previous section, if a detector for a PIT MIMO system does not use the equivalent channel matrix to find its decision variables, it will have difficulty doing symbol detection due to additional MAI created by quantization errors. In Chapters 2 and 4 we described how to build MMSE filters for symbol detection after the received samples have been filtered by the spatial filter, $\mathbf{W}$. The nulling vectors of these filters are given for the case where there is perfect CSI available at the transmitter. The solution to this problem is quite simple. That is, we match the nulling vectors of calculated from (2.16) and (4.7) to $\mathbf{G}_{P I T}$. However, in doing this we will lose some of the performance gains we profited from in implementing a precoded MIMO system. This is a small sacrifice that must be made to ensure good detector performance.

MMSE-SIC Filters for PIT The MMSE-SIC detector described in Section 2.2.3 makes use of a $\mathrm{QR}$ decomposition to find its nulling vectors. The nulling vectors, 
once chosen, do not change until the next channel realization. The nulling vectors for MMSE-SIC are computed using (2.13) - (2.16) matched to the ideal equivalent channel matrix, $\mathrm{G}_{I T}$. To match the MMSE-SIC nulling vectors to the equivalent channel matrix, $\mathbf{G}_{P I T}$, we simply substitute $\mathbf{G}_{P I T}$ for $\mathbf{G}_{I T}$ in (2.13) - (2.16).

MMSE-ISF Filters for PIT The MMSE-ISF nulling vectors are calculated using (4.7) in Chapter 4. In Chapter 4 the MMSE-ISF nulling vectors are computed with the assumption of an IT. That is, the nulling vectors are computed using $\mathbf{G}_{I T}$. With the knowledge that the equivalent channel matrix is actually $\mathbf{G}_{P I T}$, the detector should match the MMSE-ISF nulling vectors to $\mathbf{G}_{P I T}$. The appropriate MMSE nulling vectors that minimize the MSE between each decision variable and transmitted symbol vector for a PIT are computed as

$$
\mathbf{d}_{i}=\mathbf{g}_{P I T, i}^{+}\left(\mathbf{G}_{P I T} \mathbf{Q}_{s} \mathbf{G}_{P I T}^{+}+\mathbf{I}_{L} N_{0}\right)^{-1}
$$

where $\mathbf{g}_{P I T, i}$ is the $i^{t h}$ column vector of $\mathbf{G}_{P I T}$.

\subsection{Quantization of a Semi-Unitary Matrix}

In all MIMO channel decompositions presented in Section 2.3 one or two semi-unitary matrices are required. Given a PIT MIMO system, a methodology must be developed to quantize these semi-unitary precoder matrices. In this section we propose a system for quantizing a semi-unitary matrix, while trying to limit the amount of feedback required. 


\subsubsection{Characterizing a Semi-Unitary Matrix by a Set of Pa- rameters}

Any $N \times M$ complex matrix is defined by $N M$ phase and magnitude parameters. However, we can use some of the characteristics of a semi-unitary matrix to reduce the number of required parameters. In particular, we exploit the fact that $\mathbf{V}^{+} \mathbf{V}=\mathbf{I}_{L}$ for any semi-unitary matrix, $\mathbf{V}$.

We begin by representing a $N \times M$ semi-unitary matrix

$$
\mathbf{V}=\left[\begin{array}{cccc}
\left|v_{11}\right| e^{j \phi_{11}} & \left|v_{12}\right| e^{j \phi_{12}} & \cdots & \left|v_{1 M}\right| e^{j \phi_{1 M}} \\
\left|v_{21}\right| e^{j \phi_{21}} & \left|v_{22}\right| e^{j \phi_{22}} & \cdots & \left|v_{2 M}\right| e^{j \phi_{2 M}} \\
\vdots & \vdots & \ddots & \vdots \\
\left|v_{N_{1}}\right| e^{j \phi_{N 1}} & \left|v_{N_{2}}\right| e^{j \phi_{N_{2}}} & \cdots & \left|v_{N M}\right| e^{j \phi_{N M}}
\end{array}\right]
$$

as the product of two matrices

$$
\mathrm{V}=\Upsilon \Psi
$$

where $\Upsilon$ is a $N \times M$ semi-unitary matrix; and $\Psi$ is a $M \times M$ diagonal unitary matrix with entries, $\psi_{i}$, which are phases that are removed from $\mathbf{V}$ in creating $\Upsilon$. As an example, for $\mathbf{V}$ being a $2 \times 2$ matrix, $\psi_{i}=e^{\phi_{1 i}}$. The effect of $\boldsymbol{\Psi}$ is that it only applies a phase rotation on each subchannel. This phase rotation can be corrected just as easily at the receiver as the transmitter, so there is no need to send it to the transmitter. At most only $N M-M$ parameters are needed to describe $\Upsilon$. The next step is to find a way to represent the magnitudes and phases in $\Upsilon$.

Since $\Upsilon$ is a semi-unitary matrix it can be decomposed using a set of Givens 
rotations, so

$$
\Upsilon=\prod_{j=1}^{L-1} \prod_{l=2}^{N_{t}-j} \Upsilon_{j l}
$$

where each submatrix $\Upsilon_{j l}$ has the form

$$
\mathbf{\Upsilon}_{j l}=\left[\begin{array}{cccccc}
\alpha_{j l} & 0 & \cdots & \sqrt{1-\alpha_{j l}^{2}} e^{j \beta_{j l}} & \cdots & 0 \\
0 & 1 & 0 & \ddots & \ddots & \vdots \\
\vdots & \vdots & \ddots & \ddots & \ddots & \vdots \\
\sqrt{1-\alpha_{j l}^{2}} e^{-j \beta_{j l}} & 0 & \cdots & -\alpha_{j l} & \ddots & \vdots \\
\vdots & \ddots & \ddots & \ddots & \ddots & 0 \\
0 & \cdots & \cdots & \cdots & 0 & 1
\end{array}\right]
$$

where $\Upsilon_{j l}$ is an identity matrix, but for four entries. These entries are $(j, j),(j, l),(l, j)$ and $(l, l)$. In those locations we place a rotation matrix which is characterized by a single real phase $\left(\beta_{j l}\right)$ and a non-negative magnitude $\left(\alpha_{j l}\right)$ parameter. The rotation contained in each $\boldsymbol{\Upsilon}_{j l}$ matrix corresponds to each rotation obtained through the use of the Givens rotation method used in characterizing matrix $\Upsilon$. Since the total number of Givens rotations is

$$
N_{g}=\sum_{j=1}^{M-1} \sum_{l=2}^{N-j} 1=\sum_{j=1}^{M-1}(N-j-1)=M(N-1)-\frac{M(M-1)}{2}=\frac{M}{2}(2 N-M-1)
$$

we can reduce the number of parameters used in characterizing a semi-unitary matrix $\mathrm{V}$ from $2 N M$ to $\frac{M}{2}(2 N-M-1)$. For example, for a $3 \times 3$ semi-unitary matrix, the number of phase and magnitude parameters can be reduced from 18 to 6 . Using a Givens rotation characterization we have in effect cut down the amount of feedback required to quantize the matrix parameters by one-third in this example. 
With $N=N_{t}$ and $M=L$, we characterize the reduction of feedback information as

$$
G_{f}=\frac{L\left(2 N_{t}-L-1\right)}{2 L N_{t}}
$$

where if $N_{t}$ is allowed to tend toward infinity, $G_{f} \rightarrow 1$. Of course, for the purpose of this thesis we limit the number of transmit antennas to a finite number since we envision the implementation of our MIMO system for space limited devices such as laptops and wireless LANs, to name a few. For example, if $N_{t}=4, G_{f}$ ranges between

$\frac{3}{8}$ for $L=4$ and $\frac{3}{4}$ for $L=1$. It is clear that there is a diminishing return in $G_{f}$ as we increase the number of subchannels used, especially when the number of subchannels selected, $L$, is small. For the case where $L$ is small, this is not a drawback, as the amount of feedback required in total is also small. When $L \approx N_{t}, G_{f}$ is less than 0.5 for the case where we limit $N_{t}=4$. This means that the Givens rotation method of reducing the number of feedback parameters is useful for implementation into our PIT MIMO system.

\subsubsection{Parameter Quantization Strategy for a Semi-Unitary Precoder Matrix}

To quantize each of the $N_{g}$ submatrices, a real phase and magnitude must be represented using a finite set of values. By using statistical analysis, we found that for a small number of antennas $\left(N_{t} \leq 5\right)$ the phase and magnitude parameters of all Givens rotations were either negligibly or loosely correlated [16]. As a result, we assume that all phase and magnitude parameters are independent and so can be quantized independently.

Due to the unitary property of each Givens rotation the range of values that any phase or magnitude parameter can take on is fixed. All phases are distributed 
over the range $[0,2 \pi]$ and all magnitudes have range $[0,1]$. For simplicity, a uniform quantizer is used for each phase and magnitude parameter, with $\Theta$ feedback bits used to quantize each phase, and $\Omega$ feedback bits used for each magnitude.

To further reduce the number of feedback bits, the quantization range for each magnitude parameter can be reduced because of a symmetry property that exists for the Givens rotation. The non-one and -zero entries in (8.5) can be described in terms of $\alpha_{j l}$ and $\beta_{j l}$ or $\tilde{\alpha}_{j l}=\sqrt{1-\alpha_{j l}^{2}}$ and $\beta_{j l}$. We note that there is a symmetric relationship between $\alpha_{j l}$ and $\tilde{\alpha}_{j l}$ in that if $\alpha_{j l}>\frac{1}{\sqrt{2}}$ then $\tilde{\alpha}_{j l}<\frac{1}{\sqrt{2}}$ and vice versa. Using this property we can reduce the quantization range for all magnitude parameters by always transmitting the lesser of $\alpha_{j l}$ or $\tilde{\alpha}_{j l}$. The transmitted magnitude $\left(\alpha_{j l}\right.$ or $\left.\tilde{\alpha}_{j l}\right)$ is quantized using $\Omega-1$ bits, uniformly quantized over $\left[0, \frac{1}{\sqrt{2}}\right]$. An additional bit is used to indicate which of $\alpha_{j l}$ or $\tilde{\alpha}_{j l}$ was transmitted, so $\Omega$ bits are still used for each magnitude. It may seem that this extra bit increases the amount of feedback required to quantize each magnitude parameter; but on the contrary, the use of a shorter quantization interval at the expense of one bit will improve performance. This is due to the assumption that all magnitudes have uniform distribution. In reality, the magnitudes are distributed very unevenly over $[0,1]$ and a uniform quantizer over this range must use a large number of bits to represent each magnitude with sufficient accuracy. On the other hand, the magnitudes tend to be fairly uniformly distributed over $\left[0, \frac{1}{\sqrt{2}}\right]$, and only a few bits will suffice for a uniform quantizer over this range. As a result we expect much better quantizer performance using this quantization strategy. We call the parameterized quantization strategies $\mathrm{PQL}$ for the longer quantization interval and $\mathrm{PQ}$ for the shorter one. 


\subsection{Uncoded BER Performance of PIT MIMO}

For the PIT MIMO system, the desire is to reduce the amount of CSI feedback required while still maintaining good operating performance. That is, we wish to feed back as little information as possible while achieving close to the performance provided by an IT MIMO system. In the previous section we introduced a method to quantize semi-unitary precoder matrices using a set of Givens rotations. We also derived appropriate MMSE filters that are matched to the quantization errors that occur for a PIT. In this subsection we examine the how much feedback is necessary to achieve near-perfect CSI BER performance.

Three quantization strategies for representing a $N_{t} \times L$ semi-unitary matrix have been discussed: one where all entries are quantized (AEQ); one where the semi-unitary matrix is represented using a set of Givens rotations and quantization is done on each parameter in each rotation where the magnitude range is taken to be $[0,1](\mathrm{PQL})$; and one which is $\mathrm{PQL}$, but with a quantization strategy that reduces the range of quantization for each magnitude (PQ). In Section 8.2.2, we also introduced some adaptations to the hard and soft MMSE nulling vectors. We required that the nulling vectors of the detector be matched to the equivalent channel matrix, which has quantization errors, as opposed to the ideal equivalent channel matrix that would result for an IT. In this subsection we explore the uncoded BER performance of each quantization strategy with and without the matched MMSE nulling vectors and strive to determine how to best choose the amount of feedback required for acceptable system performance. We evaluate the BER performance of the PIT GMD transceiver using 16-QAM and $L=N_{t}$.

Figure 8.1 shows the performance of AEQ with a $2 \times 2$ GMD-SIC transceiver. The 


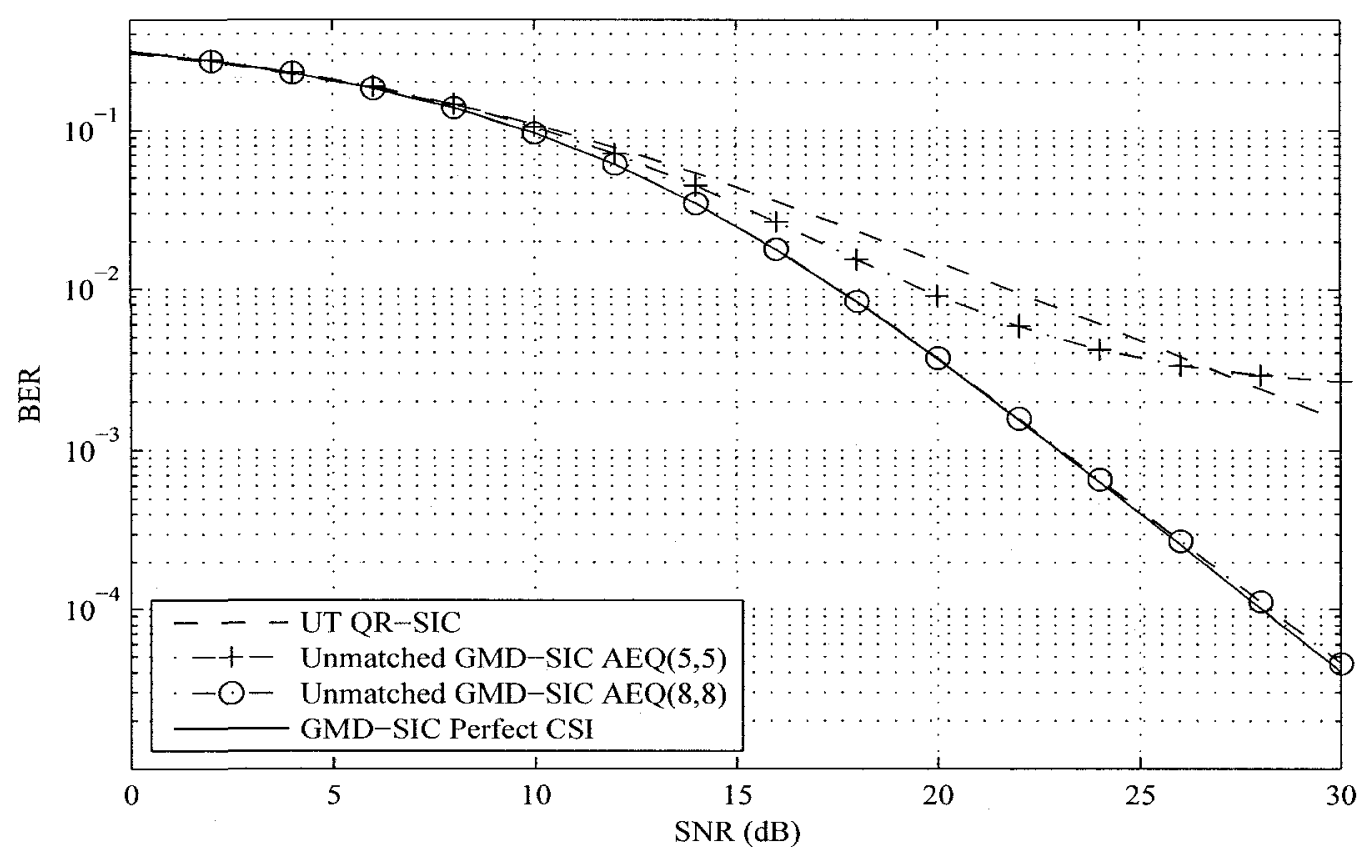

Figure 8.1: BER performance of $2 \times 2$ MIMO systems with different quantization strategies and SIC done using the ideal equivalent channel matrix.

GMD-SIC transceiver does not use nulling vectors and assumes that the equivalent channel is $\mathbf{G}_{I T}$ and, as a result, is not able to match to the equivalent channel matrix and properly do SIC. The bracketed numbers stand for the number of feedback bits used to quantize each magnitude and phase parameter. That is, $(\Omega, \Theta)$ bits. For a $2 \times 2$ MIMO system, $\operatorname{AEQ}(5,5)$ requires 40 bits to quantize the precoder matrix. Even with such a large amount of precoder feedback AEQ $(5,5)$ has terrible performance, as it barely performs better than the UT QR-SIC scheme at low SNRs and is much worse at high SNRs due to the presence of an error floor of about $3 \times 10^{-3}$. Near-IT performance can be attained when 64 bits are used to represent the precoder matrix as demonstrated by the AEQ $(8,8)$ curve in Figure 8.1. Of course, this is a lot of feedback and, for larger precoder matrices, will grow quickly. For example, supposing 8 bits are needed to get near-IT performance for any number of transmit and receive antennas, the total amount of quantization information rises to 144 and 256 bits for 


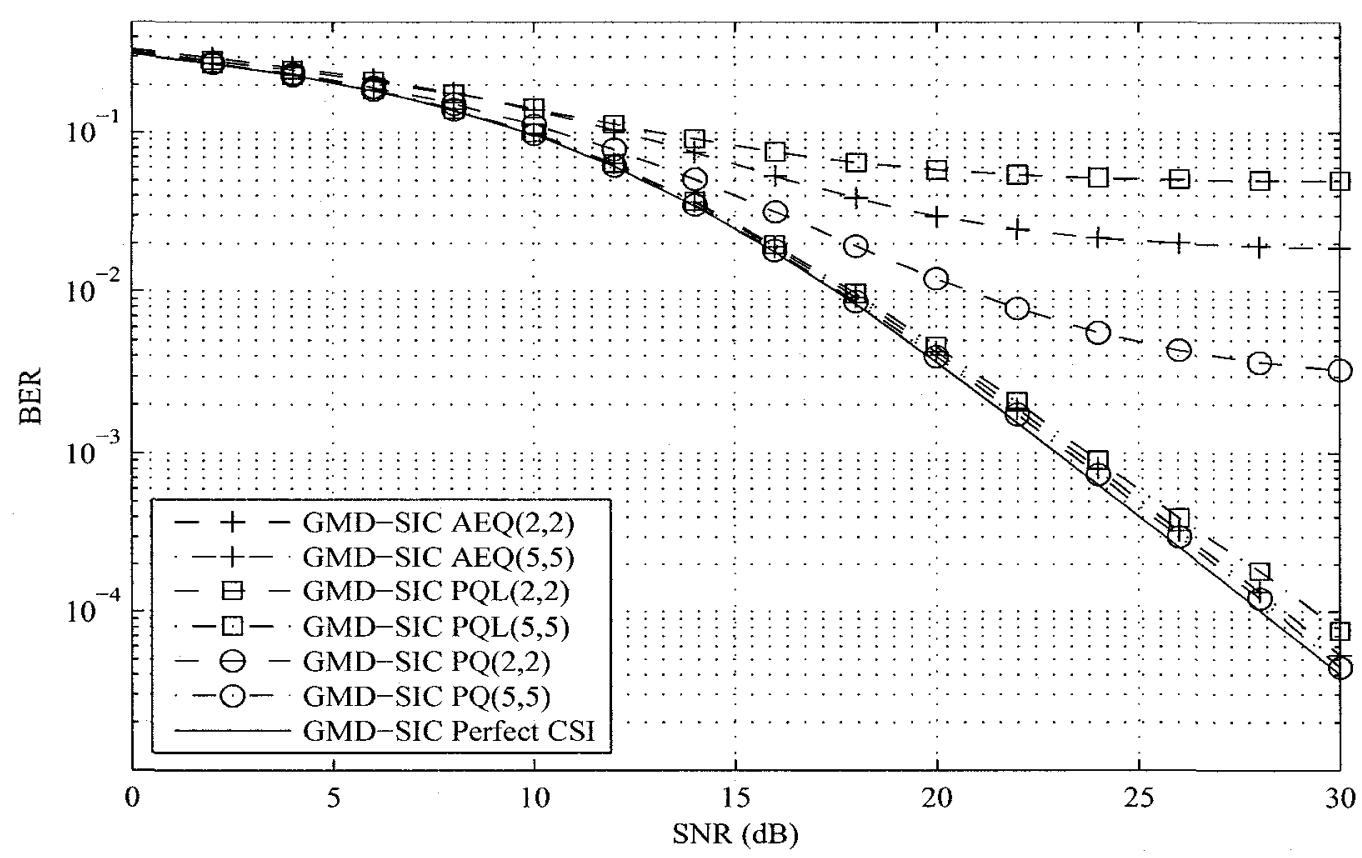

Figure 8.2: BER performance of a $2 \times 2$ GMD-SIC transceiver with different quantization strategies and SIC done using the equivalent channel matrix.

3 and 4 transmitters respectively.

A more efficient and intelligent quantizer design coupled with a detector matched to the non-ideal equivalent channel matrix reduces the amount of feedback required to achieve near-IT BER performance. Although the GMD-SIC detector is not able to null the MAI added by quantization errors, it can do better SIC when the detector matches to the equivalent matrix, $\mathbf{G}_{P I T}$. Comparing the $\operatorname{AEQ}(5,5)$ curves from Figures 8.1 and 8.2 shows that just the addition of a matched detector greatly improves the performance of the PIT MIMO system. With this addition, the $\mathrm{AEQ}(5,5)$ is able to come within $0.58 \mathrm{~dB}$ of IT BER performance at a BER of $10^{-4}$, whereas before it exhibited an error floor of $3 \times 10^{-3}$. Figure 8.2 also demonstrates that the Givens-rotation-based quantizers are able to reduce the amount of feedback information required. Though $\mathrm{PQL}(2,2)$ has a higher error floor than $\mathrm{AEQ}(2,2)$, 


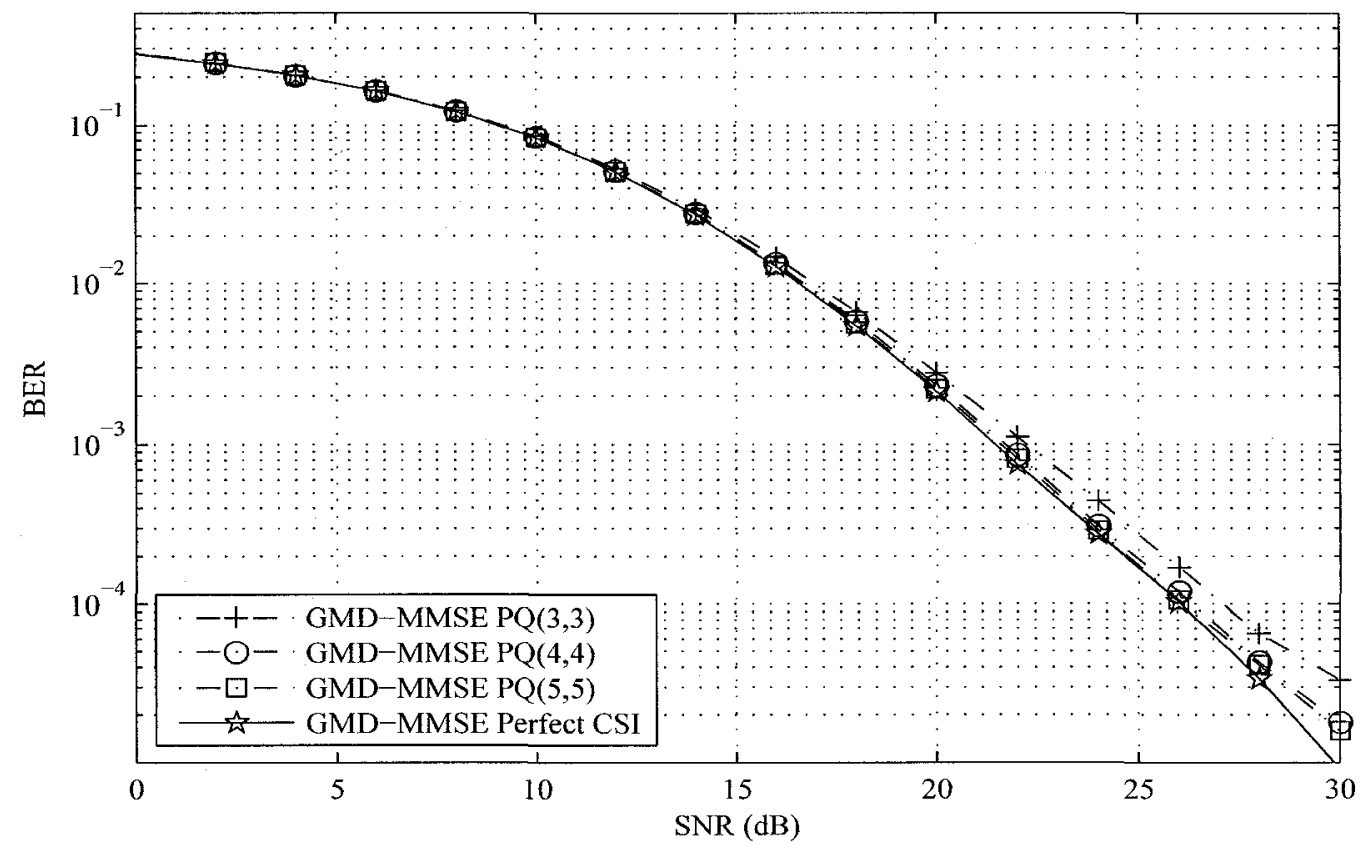

Figure 8.3: BER performance of a $2 \times 2$ GMD-MMSE with different levels of quantization with hard MMSE-SIC nulling vectors matched to the equivalent channel matrix.

it still achieves this performance with less feedback information. PQL $(2,2)$ only requires 4 bits for its quantizer, whereas $\operatorname{AEQ}(2,2)$ requires 16. A fairer comparison can be made between PQL(5,5) and AEQ $(2,2)$ (10 bits vs. 16 bits). PQL(5,5) provides a BER of $10^{-4}$ that has a $1.5 \mathrm{~dB}$ loss on the IT system. Clearly, PQL is a big improvement on AEQ. Figure 8.2 also confirms that PQ provides an additional gain over PQL. The PQ $(2,2)$ quantizer has a lower error floor than both the AEQ $(2,2)$ and PQL(2,2), and the PQ(5,5) quantizer allows the PIT MIMO system to achieve a BER of $10^{-4}$ at a SNR that is $0.33 \mathrm{~dB}$ greater than the IT MIMO system with only 10 bits of feedback. This is much less than the 40 bits required by the AEQ quantizer, and the $1.5 \mathrm{~dB}$ loss of the PQL quantizer. The PQ quantizer with matched detector is the best quantizer choice of these three options and is used in all further simulations.

Figure 8.3 shows the BER performance of PQ for a $2 \times 2$ GMD-MMSE transceiver 


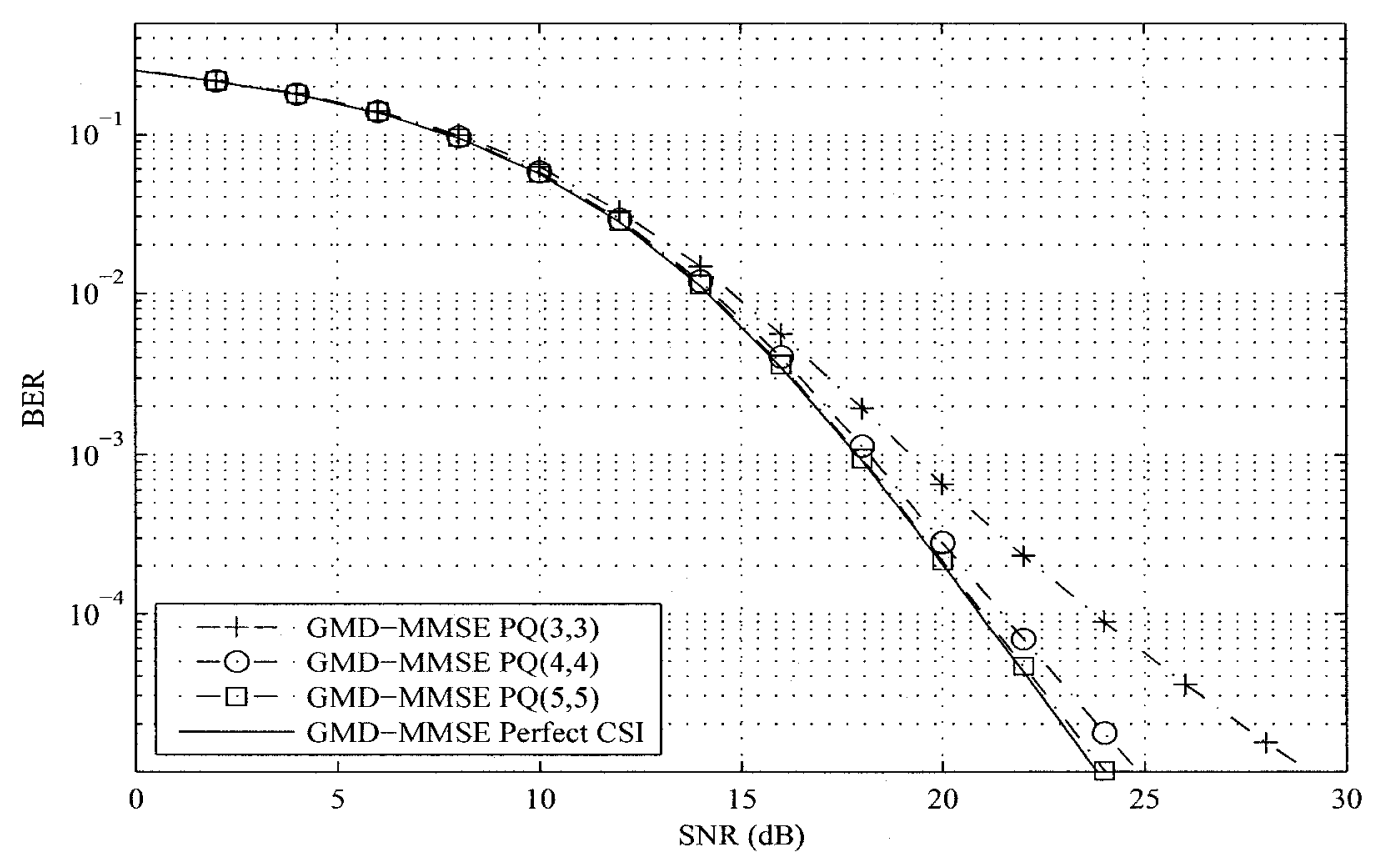

Figure 8.4: BER performance of a $3 \times 3$ GMD-MMSE with different levels of quantization with hard MMSE-SIC nulling vectors matched to the equivalent channel matrix.

with MMSE-SIC nulling vectors matched to $\mathbf{G}_{P I T}$. The figure demonstrates that using MMSE nulling vectors and SIC matched to the equivalent channel matrix removes the error floor exhibited when SIC detection is used without MAI nulling. This is because the MMSE nulling vectors are able to suppress the added interference created by quantization errors. We see that a matched MMSE-SIC detector with a PQ quantizer has an SNR loss at $10^{-4}$ on the IT MIMO system of about $1.08 \mathrm{~dB}$ for $\mathrm{PQ}(3,3) ; 0.25 \mathrm{~dB}$ for $\mathrm{PQ}(4,4)$; and $0.08 \mathrm{~dB}$ for $\mathrm{PQ}(5,5)$.

Figure 8.4 gives the BER performance for the $3 \times 3$ PIT GMD-MMSE transceiver. The SNR performance loss at $10^{-4}$ on the IT GMD-MMSE transcciver is about $3.2 \mathrm{~dB}$ for $\mathrm{PQ}(3,3) ; 0.6 \mathrm{~dB}$ for $\mathrm{PQ}(4,4)$; and $0.1 \mathrm{~dB}$ for $\mathrm{PQ}(5,5)$. The amount of feedback bits required to quantize a $3 \times 3$ semi-unitary matrix is now $3 \times(\Omega+\Theta)$, whereas for the $2 \times 2$ semi-unitary matrix it was only $\Omega+\Theta$. 


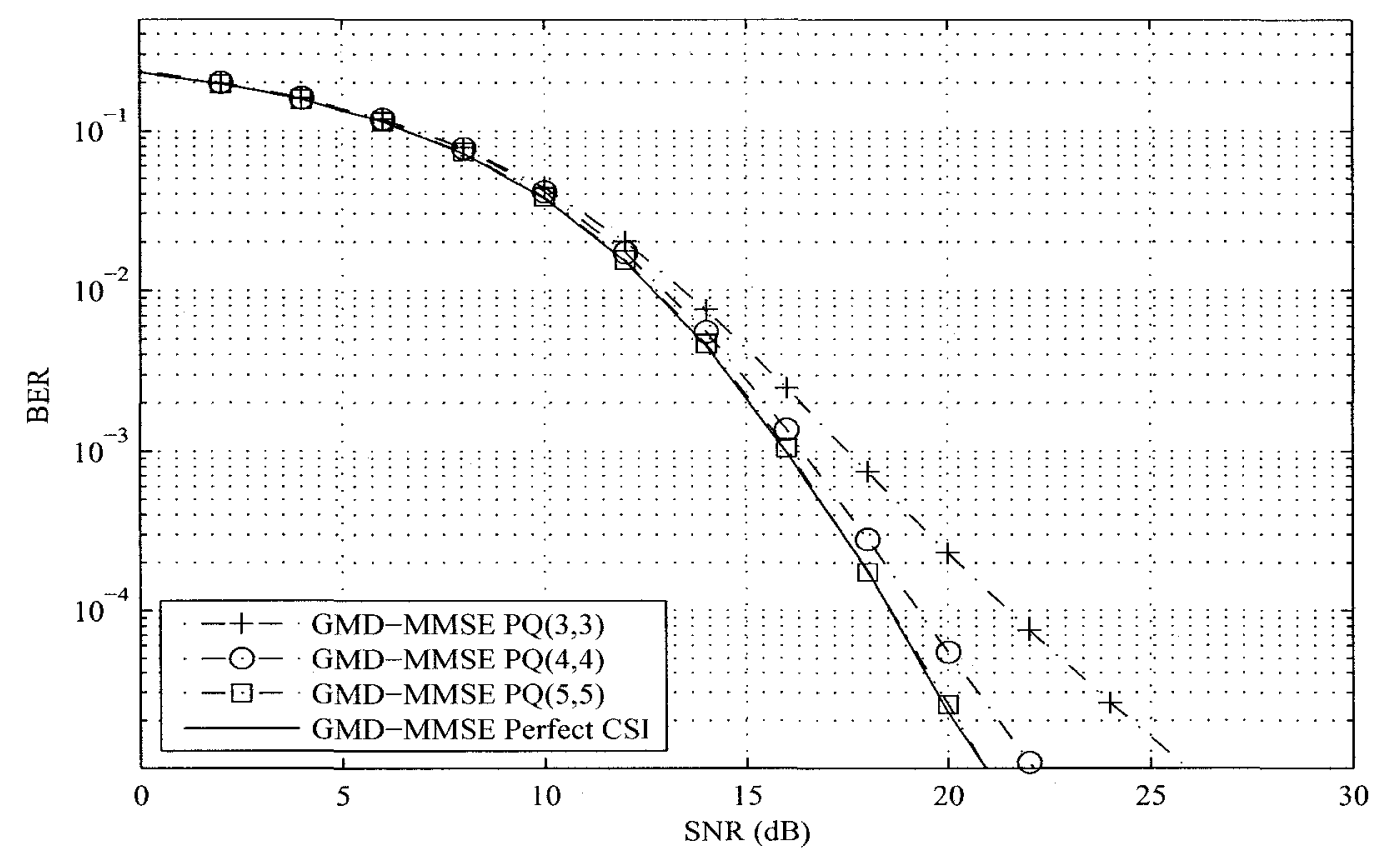

Figure 8.5: BER performance of a $4 \times 4$ GMD-MMSE with different levels of quantization with hard MMSE-SIC nulling vectors matched to the equivalent channel matrix.

Figure 8.5 shows the BER performance for the $4 \times 4$ GMD-MMSE transceiver for different amounts of feedback information. The SNR loss at a BER of $10^{-4}$ on the IT GMD is $3.2 \mathrm{~dB}$ for $\mathrm{PQ}(3,3) ; 0.8 \mathrm{~dB}$ for $\mathrm{PQ}(4,4)$; and is negligible for $\mathrm{PQ}(5,5)$. This means that for four transmit and receive antennas the PQ $(5,5)$ quantizer is essentially able to achieve IT performance. In the case of the $4 \times 4$ transceiver we must feed back $6 \times(\Omega+\Theta)$ bits, which is double the number of bits for the $3 \times 3$ transceiver.

The BER performance of the PQ quantizer with matched detector has been determined for a variety of numbers of bits; however, the importance of each parameter - the magnitude and phase - has not. The question is whether or not more feedback information should be put into one parameter or whether it is better to use the same amount of feedback information for both. 
Figures 8.6 - 8.8 are used to give an indication of how to assign feedback bits in characterizing a semi-unitary matrix. The main purpose of the magnitude information is to steer the matrix energy into the appropriate rows and columns of the equivalent matrix in order to create the desired structure of the matrix. If there is quantization error in the magnitude, there will be energy left unsteered in parts of the equivalent channel matrix that create MAI. The purpose of the phase is to ensure that entries become real in the equivalent channel matrix. If a phase is off, the next Givens rotation that is done on the matrix will also be off, creating more MAI due to quantization errors. A difference between the magnitude and phase parameters is that they have different quantization ranges, with the phase having the larger of the two. This may suggest that using more phase information is more important due to the large range over which it must be quantized.

Figure 8.6 gives the performance of the $2 \times 2$ transceiver for $\operatorname{PQ}(2,4), \operatorname{PQ}(3,3)$ and PQ(4,2), or when 6 bits are allocated to each Givens rotation. It is easy to see that PQ(3,3) performs the best. Second best is PQ(4,2), suggesting that the magnitude information is more important for the $2 \times 2$ GMD-MMSE transceiver.

Figure 8.7 gives the BER performance of a $3 \times 3$ GMD-MMSE transceiver for the same set of parameter as in Figure 8.6. Again, the best performer is $\mathrm{PQ}(3,3)$, but this time the second best is $\mathrm{PQ}(2,4)$, which suggests that phase information is more important for the $3 \times 3$ transceiver.

Figure 8.8 gives the BER performance of a $4 \times 4$ PIT GMD-MMSE transceiver. Using the same parameters as in Figures 8.6 and 8.7 we see that the best performer is $\mathrm{PQ}(3,3)$. The second best performer is $\mathrm{PQ}(2,4)$, but in this figure the gap between 


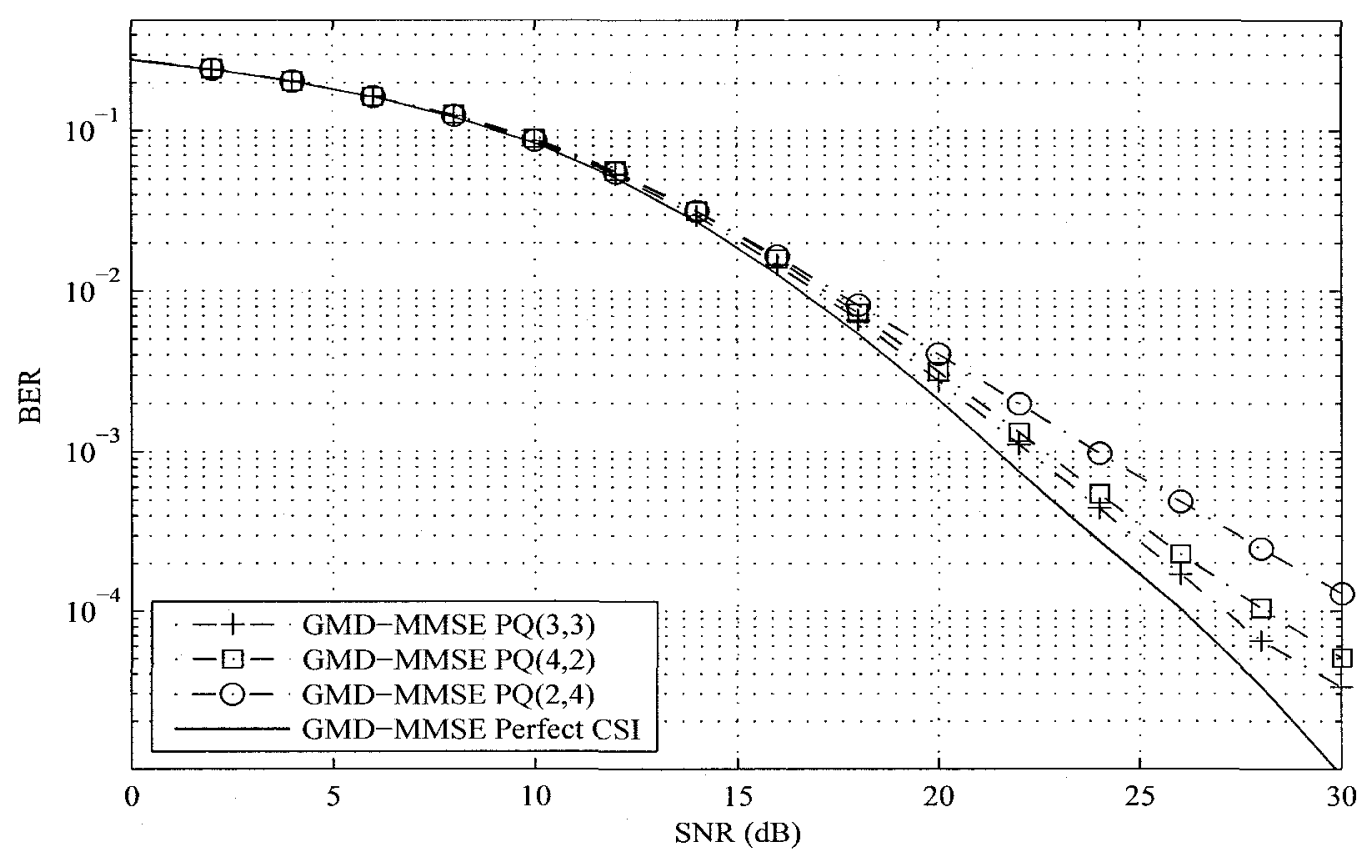

Figure 8.6: BER performance of a $2 \times 2$ GMD-MMSE with a MMSE-SIC detector matched to the equivalent channel, comparing the importance of magnitude versus phase information.

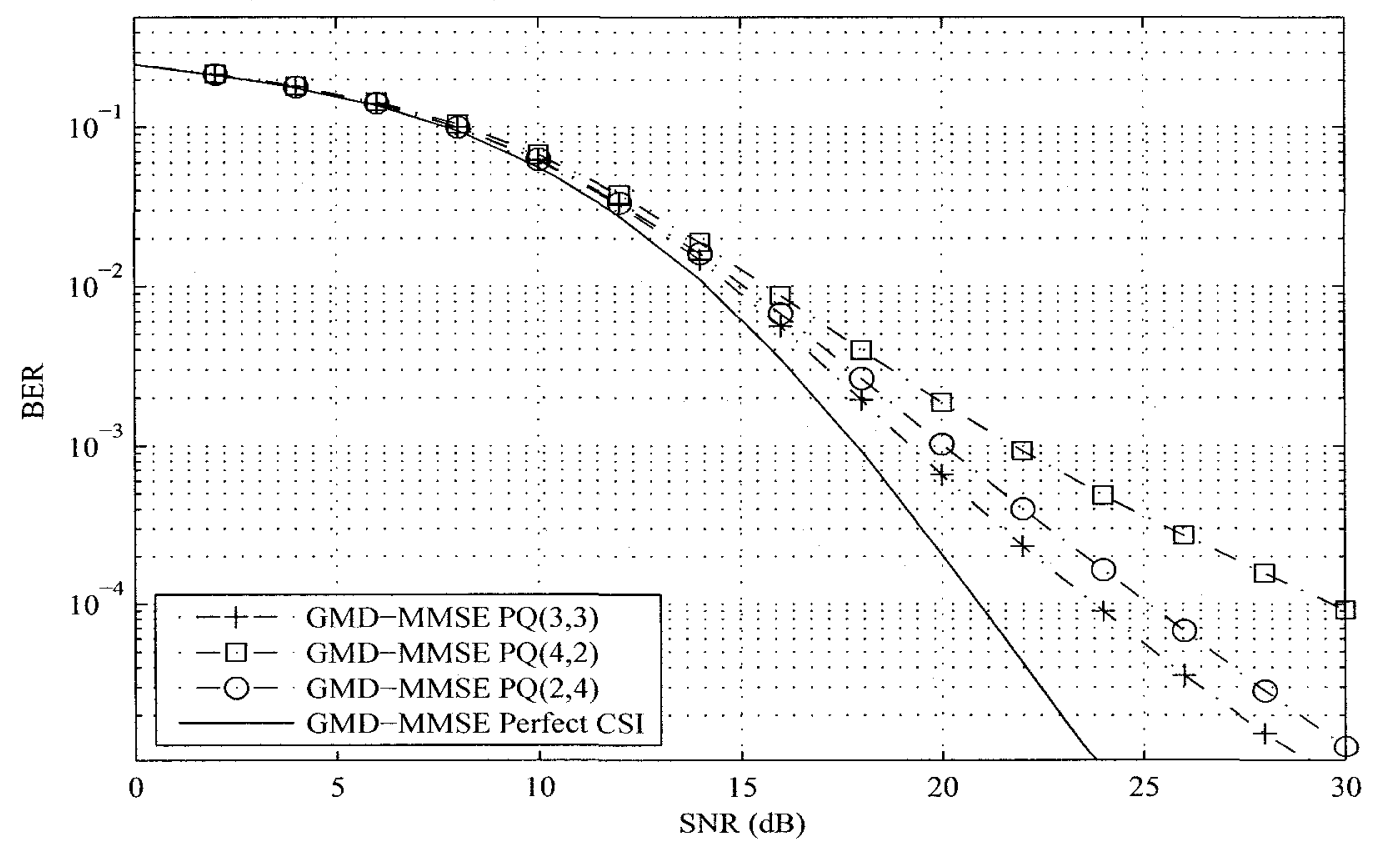

Figure 8.7: BER performance of a $3 \times 3$ GMD-MMSE with a MMSE-SIC detector matched to the equivalent channel, comparing the importance of magnitude versus phase information. 


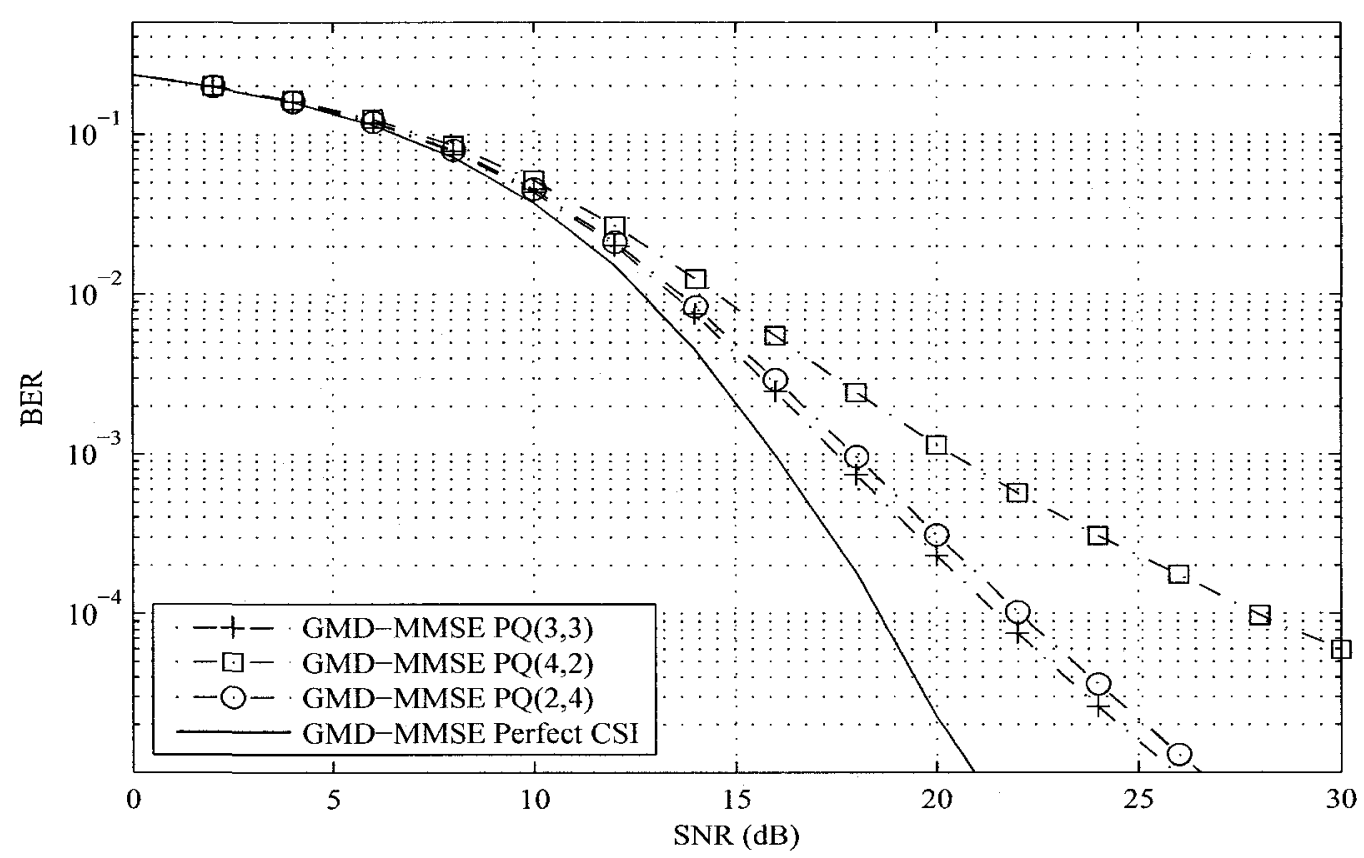

Figure 8.8: BER performance of a $4 \times 4$ GMD-MMSE with a MMSE-SIC detector matched to the equivalent channel, comparing the importance of magnitude versus phase information.

the best and second best performer has closed and the gap between the best and worst performer has widened. These results suggest that for PIT GMD-MMSE transceivers with the same number of transmit and receive antennas, and with this number being greater than two, the phase information is the most important. However, from all figures it is clear that if we can divide the number of feedback bits evenly, we should. An explanation for the behaviour of the $2 \times 2$ PIT GMD-MMSE is that the phase information does not affect part of the equivalent channel matrix. That is, because the phases in the first Givens rotation does not impact any other Givens rotations as there is only one applied rotation for the $2 \times 2$ case - there is more impact from the magnitude. Of course, the equivalent channel matrix, $\mathbf{G}_{P I T}$, will not be perfect, as the phase, once chosen, does impact the choice of magnitude. Without the proper phase, some energy is not steered to the desired locations and MAI is still created. 


\subsection{Experimental Throughput Performance of PIT RC/QC-MIMO}

In Section 8.4 we demonstrated the BER performance of the PQ quantizer for various amounts of feedback and showed that near-perfect CSI performance can be approached with five bits per quantization parameter for a GMD transceiver matched to the equivalent channel. This transceiver was just one of four of the remaining transceivers that we are still evaluating at this point in our study. We have yet to evaluate the performances of the SVD, ASGMD and Hybrid transceivers for limited amounts of precoder feedback. This section gives simulation results for the aforementioned transceivers (including GMD) in order to see the impact that a PIT using PQ with MMSE-ISF nulling vectors matched to the equivalent channel matrix has on the throughput performance of each system. All transceivers use the CWFA to do bit-rate allocation on each subchannel, a T-SS/LLRE with $N_{I}=1$ and $L_{z}=3$ unless specified otherwise. For RC/QC-Hybrid systems look-up tables are generated using the throughput performances of RC/QC-ASSVD PQ $(3,3)$ and RC/QC-ASGMD PQ(3,3) for a normalized channel.

Figure 8.9 demonstrates the throughput performance of RC/QC-SVD transceiver with PQ(3,3). A loss in performance is seen for the SVD transceiver when perfect CSI is not available. On the third iteration of detection, there is a $2 \mathrm{~dB}$ gain over the first for a spectral efficiency of $10 \mathrm{bps} / \mathrm{Hz}$. The fact that there is a gain between the first and third iteration means that there is a loss in performance when a PQ quantizer is used for the SVD transceiver. Additionally, these results show that the MMSE-ISF is capable of regaining some of the performance that is lost when using a PIT RC/QC-MIMO system. 


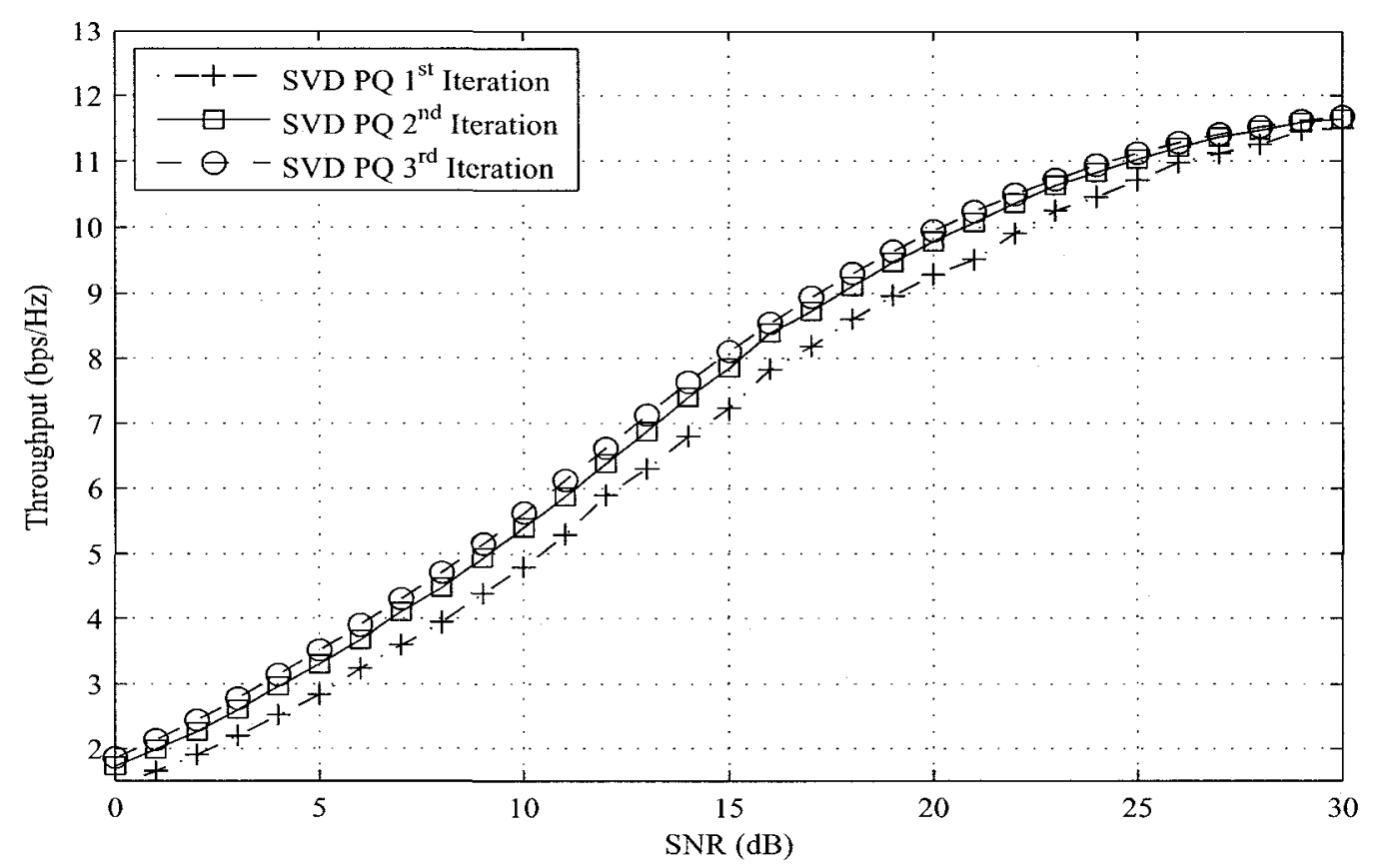

Figure 8.9: Experimental throughput performance of $2 \times 2$ SVD throughput performance with PQ quantizer.

Figure 8.10 demonstrates the performance loss of the $2 \times 2$ SVD, GMD-MMSE and ASGMD-MMSE transceivers when the $P Q$ quantizer is used to quantize their precoder matrices with three bits per magnitude and phase parameter. The RC/QC-SVD, RC/QC-GMD and RC/QC-ASGMD transceivers do not incur any significant loss when coupled with the $\mathrm{PQ}(3,3)$ quantizer as all PIT curves hug the IT performance curves. These results a very encouraging. They suggest that even less than five bits of feedback per magnitude and phase parameter can be used in characterizing the semi-unitary precoder matrix, while still achieving near-IT performance.

Figure 8.11 gives the throughput performance of $2 \times 2$ RQ/QC-Hybrid with 


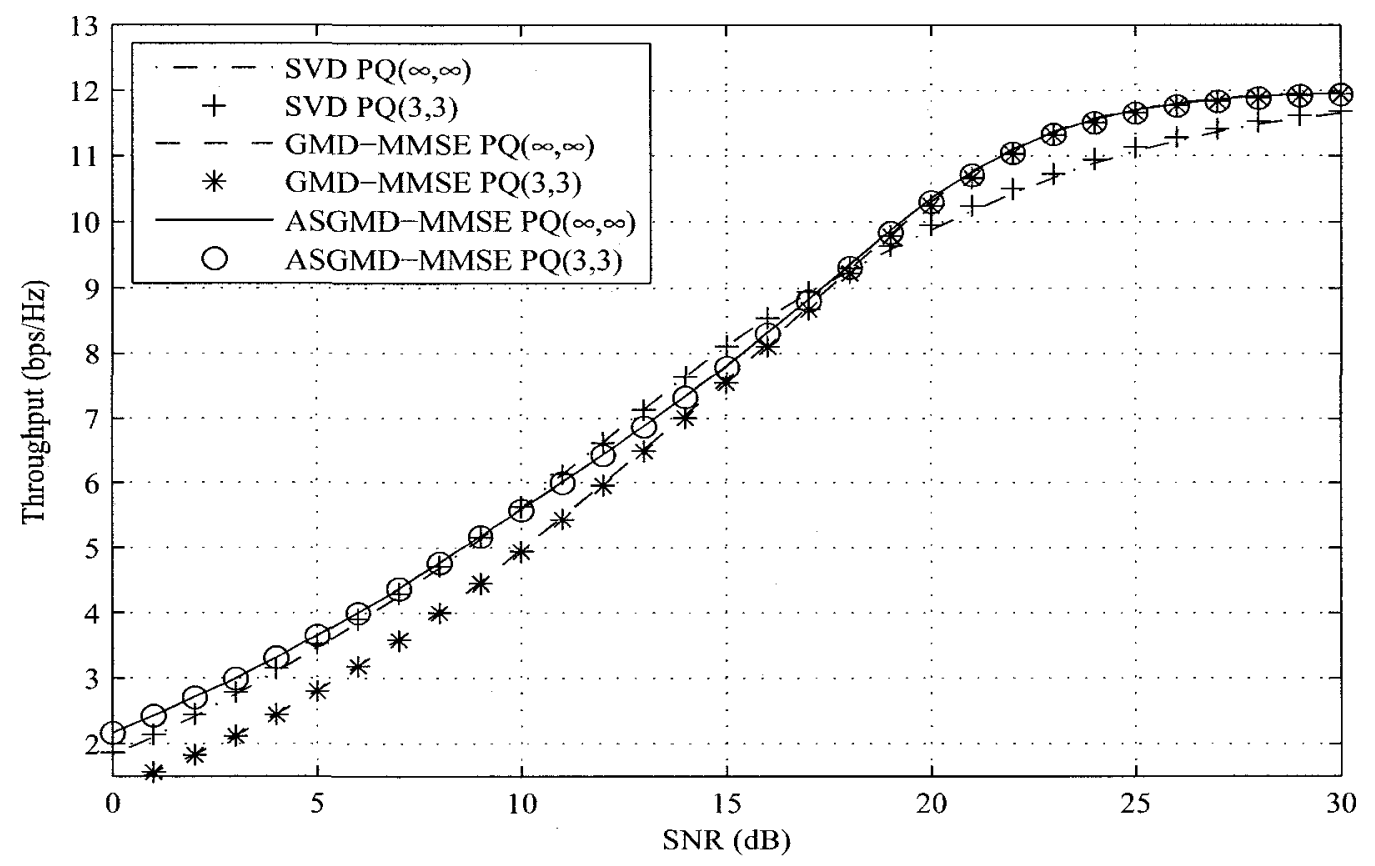

Figure 8.10: Performance degradation for $2 \times 2$ transceivers using PQ quantizer.

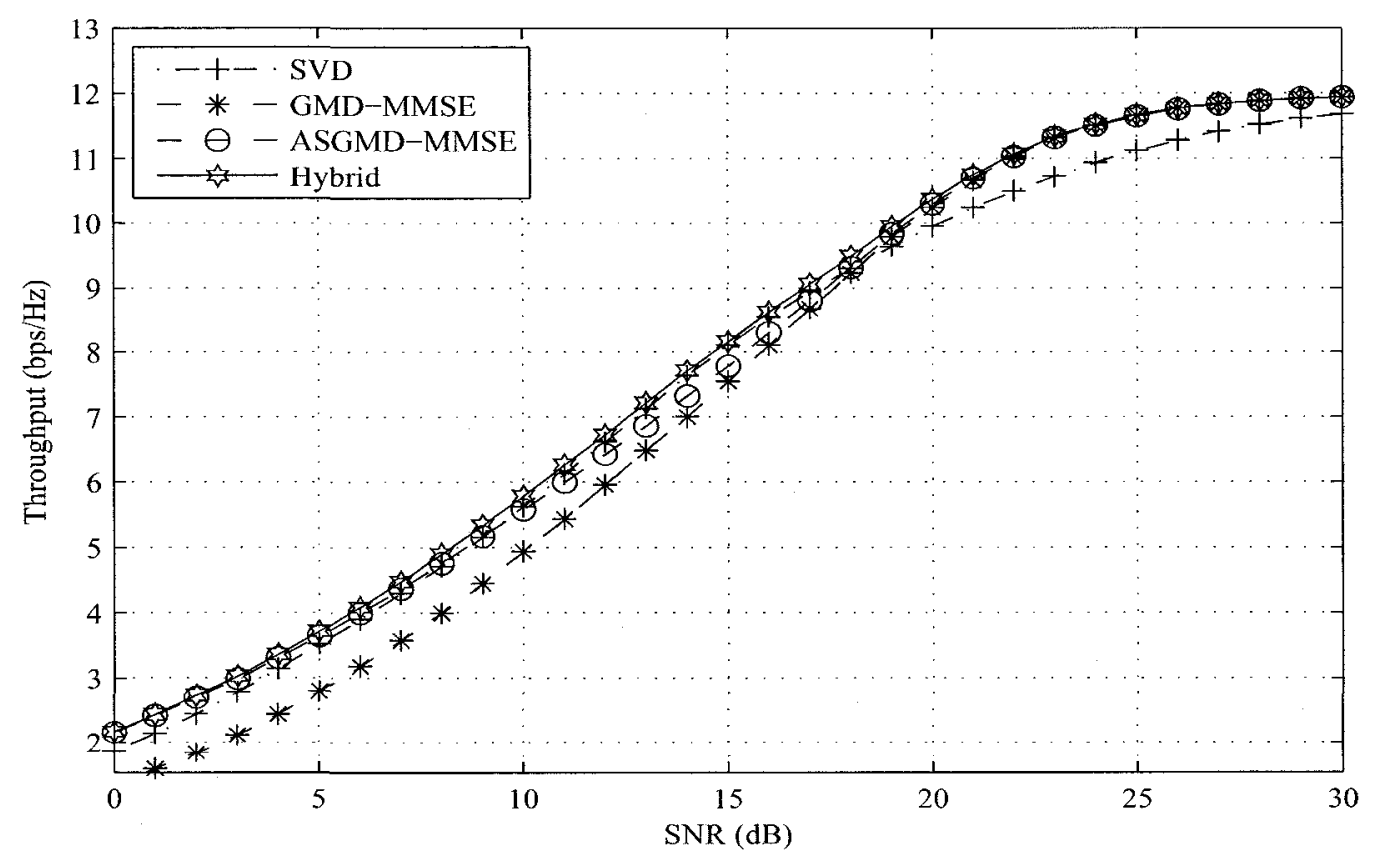

Figure 8.11: Experimental throughput performance of $2 \times 2$ transceivers using $P Q$ quantizer. 


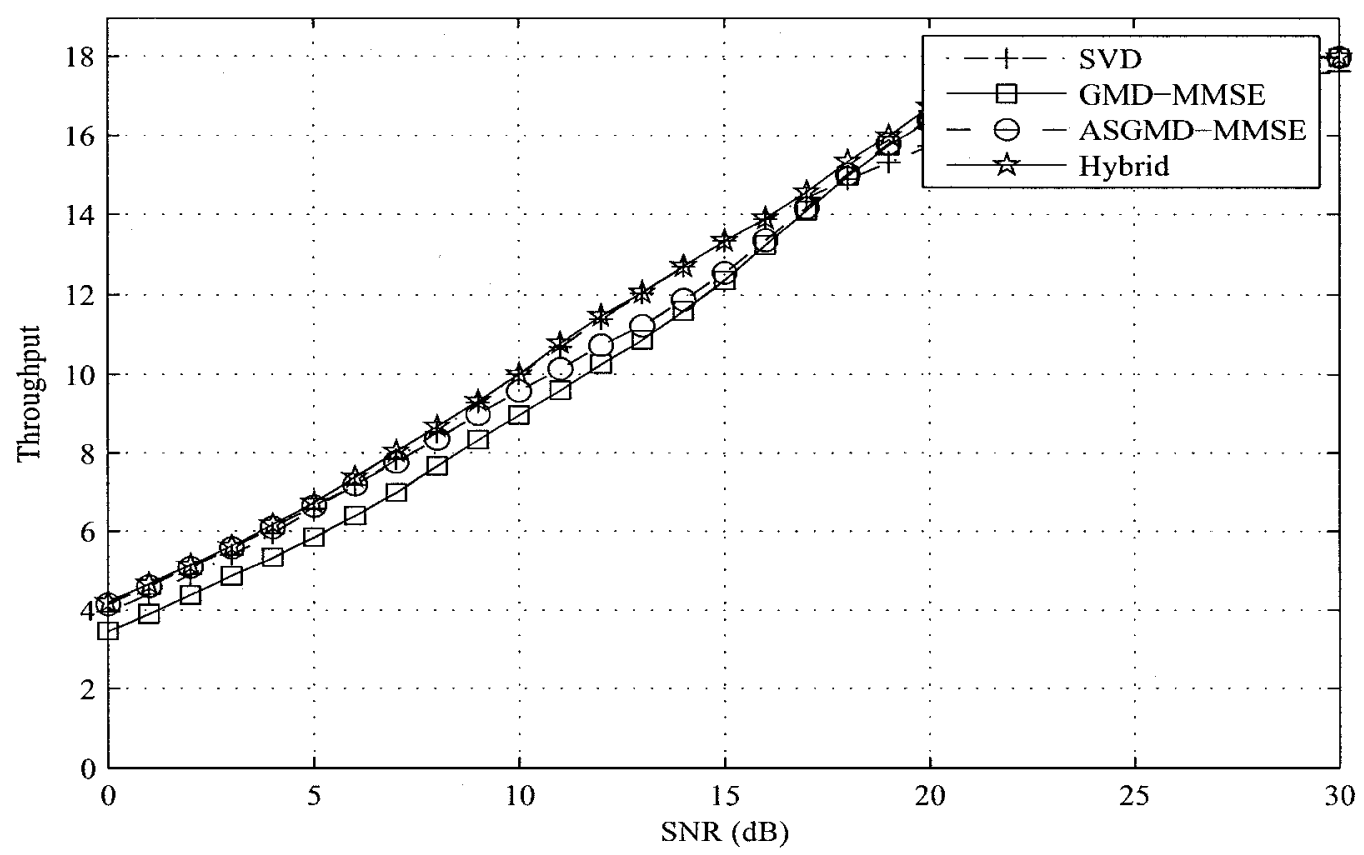

Figure 8.12: Experimental throughput performance of $3 \times 3$ transceivers using $\mathrm{PQ}$ quantizer.

PQ(3,3). We see that, as it did in Chapter 7, the RC/QC-Hybrid has better throughput performance than RQ/QC-SVD and RC/QC-ASGMD-MMSE for all SNRs. Figure 8.12 gives the throughput performance of the $3 \times 3 \mathrm{RC} / \mathrm{QC}-\mathrm{MIMO}$ system, with $\mathrm{PQ}(3,3)$. The results shown in this figure complement those given in Figure 8.11. 


\section{Chapter 9}

\section{Conclusions}

\subsection{Conclusions}

This thesis studies an implementation of RC/QC LDPC codes with rate-constrained linear MIMO transceivers. We presented a comprehensive study of channel decomposition-based MIMO transceivers to better understand their performance capabilities. While some of these systems have been studied for one or two of these criteria simultaneously, to our knowledge they have not all been compared together using all three criteria. This study revealed that although a MIMO transceiver may have good unconstrained capacity and diversity at higher SNRs it may not have good constrained throughput.

$\mathrm{RC} / \mathrm{QC}-\mathrm{MIMO}$ is the implementation of a wireless communication system that combines the MIMO transceivers described in this thesis with RC/QC LDPC codes. $\mathrm{RC} / \mathrm{QC}-\mathrm{MIMO}$ as implemented in this thesis also includes the implementation of the contributions listed below. RC/QC-MIMO is a useful system that allows a wireless communication system to enjoy the high spectral efficiency of MIMO, while still being able to adapt to the channel fluctuations of the wireless medium with low overhead. 
The WFA is appropriate to use for power and bit-rate allocation for communication systems with unconstrained throughput; however this was not to be true for rate-constrained MIMO systems. The CWFA was designed to do power and bit-rate allocation for the RC/QC-MIMO system and was shown to provide better throughput performance than when the WFA is used. In addition, the CWFA bit-rate allocator gave better experimental throughput performance than when only a 64-QAM constellation is used to modulate the code bits.

A new Tunable SSE/LLRE was designed for layered BLAST detection. TSSE/LLRE is adaptable to the MAI contained in a decision variable and is capable of considering the statistics of a variable number of interfering symbols in determining the soft-symbol and LLR estimates. T-SSE/LLRE was shown capable of achieving diversity for $N_{I}>1$. Furthermore, T-SSE/LLRE is adaptable and can be adjusted to reduce its computational complexity for a given instantaneous SNR. The experimental throughput performance results showed that T-SSE/LLRE has better performance than when a SSE/LLRE that assumes all MAI to be Gaussian is used.

The experimental throughput results for RC/QC-MIMO showed that RC/QCASGMD has good low/high SNR performance and that RC/QC-SVD has good mid-range SNR performance. These results prompted the design of the Hybrid MIMO transceiver, which was implemented for no additional computational complexity. This Hybrid transceiver was designed using the combination SVD and GMD transceivers coupled with antenna selection. This transceiver, when combined with RC/QC LDPC codes, achieved the best overall throughput performance of all those studied. 
This thesis also considered the RC/QC-MIMO for the case were limited CSI is available at the transmitter. A new parameterized quantization method, PQ, for a semi-unitary precoder was proposed. PQ operates using a set of Givens rotations to represent a semi-unitary precoder matrix. Results demonstrated that near-perfect CSI performance can be achieved by using at most five bits per phase and magnitude parameter for an $N \times N$ MIMO transceiver. The experimental throughput results also showed that the RC/QC-GMD-based systems have a smaller spectral efficiency loss than RC/QC-SVD-based systems do for a limited amount of precoder feedback.

A constrained-rate antenna selection algorithm was proposed for ASGMD. The CCAS was shown to perform better with RC/QC-ASGMD than the UCAS algorithm. This was shown in terms of the theoretical constrained throughput and the experimental throughput results.

\subsection{Future work}

This section describes suggested future work that could be done to further this study.

- The RC/QC-MIMO system was studied using the average realizable rate. It would be useful to compare the performance of the RC/QC-MIMO systems using outage capacity.

- Future studies could compare the performance of a MCW implementation with the SCW implementation.

- A study could be done to find the best way to allocation rate and power to each MIMO subchannel for the SCW implementation.

- An optimal transceiver has yet to be derived for the rate-constrained RC/QCMIMO system. 
- A study of the effects of a practical CRC code on the throughput performance of LDPC-MIMO could be done. A real CRC code could be implemented in tandem with the $\mathrm{BP}$ decoder to give a better idea of the impact that a CRC has on RC/QC-MIMO throughput.

- A quantizer for the power allocation matrix still needs to be derived.

- A quantizer based upon the pdf of each magnitude and phase parameter of each Givens rotation can be found. This would be useful for large antenna arrays, where the amount of feedback required to quantize a semi-unitary matrix becomes large. 


\section{List of References}

[1] I. Telatar, "Capacity of multiple antenna gaussian channels," ATEST Technical Memorandum, June 1995.

[2] S. Alamouti, "A simple transmit diversity technique for wireless communications," IEEE Journal on Selected Areas in Communications, vol. 16, pp. 14511458, October 1998.

[3] V. Tarokh, N. Seshadri, and A. Calderbank, "Space-time codes for high data rate wireless communications: Performance criterion and code construction," IEEE Transactions on Information Theory, vol. 44, pp. 744-765, March 1998.

[4] V. Tarokh, A. Naguib, N. Seshadri, and A. Calderbank, "Combined array processing and space-time coding," IEEE Transactions on Information Theory, vol. 47, pp. 199-207, February 1999.

[5] V. Tarokh, H. Jafarkhani, and A. Calderbank, "Space-time block codes from orthogonal design," IEEE Transactions on Information Theory, vol. 45, pp. 1456 1467, July 1999.

[6] G. Foschini, Jr., "Layered space-time architecture for wireless communication in a fading environment when using multi-element antennas," Bell Labs Tech. Journal, vol. 10, no. 6, pp. 585-595, 1995.

[7] G. Foschini, Jr., G. Golden, R. Valenzuela, and P. Wolniansky, "Simplified processing for high spectral efficiency wireless communication employing multipleelement arrays," IEEE Journal on Selected Areas in Communications, vol. 17, pp. 1841-1852, November 1999.

[8] L. Zheng and D. Tse, "Diversity and multiplexing: A fundamental tradeoff in multiple-antenna channels," IEEE Transactions on Information Theory, vol. 49, pp. 1073-1096, May 2003. 
[9] G. Raleigh and J. Cioffi, "Spatio-temporal coding for wireless communication," IEEE Transactions on Communications, vol. 46, pp. 357-366, March 1998.

[10] Y. Jiang, Transceiver design for MIMO communications: a channel decomposition perspective. $\mathrm{PhD}$ thesis, University of Florida, April 2005.

[11] M. Sellathurai and S. Haykin, "Turbo-BLAST for wireless communications: Theory and experiments," IEEE Transactions on Signal Processing, vol. 50, pp. 2538-2545, October 2002.

[12] H. Futaki and T. Ohtsuki, "Low-density parity-check (LDPC) coded MIMO systems with iterative turbo decoding," in IEEE 58th Vehicular Technology Conference, vol. 1, (Orlando, FL), pp. 342-346, October 2005.

[13] Z. He, S. Roy, and P. Fortier, "Adaptive LDPC codes for MIMO transceiver with adaptive spectral efficiency," in 24th Biennial Symposium on Communications, pp. 120-123, June 2008.

[14] Y. Zhang, D. Yuan, T. Han, and F. Zhang, "Cross layer adaptive transmission in communication systems," in Chinese Control and Decision Conference 2008, pp. 3486-3489, July 2008.

[15] 3GPP2: Physical Layer for Ultra Mobile Broadband (UMB) Air Interface Specification, 2007. Available [Online]: http://www.3gpp2.org/Public html/Misc/C.P0084- 001 v1.088 PHY clean VV Due 23 Aug-2007.pdf.

[16] M. Dorrance and I. Marsland, "Robust transceiver design for geometric mean decomposition systems with limited precoder feedback," in CCECE 2008, pp. 951956, May 2008.

[17] D. Tse and P. Viswanath, Fundamentals of Wireless Communications. Cambridge Press, 2005.

[18] K. Liu and A. Sayeed, "An iterative extension of BLAST decoding algorithm for layered space-time signals," IEEE Transactions on Communications, vol. 53, pp. 1764-1761, October 2005.

[19] B. Hassibi, "A fast square-root implementation for BLAST," in Thirty-Fourth Asilomar Conf. on Signals and Computers, pp. 1255-1259, November 2000.

[20] Y. Jiang, J. Li, and W. Hager, "Joint transceiver design for MIMO communications using geometric mean decomposition," IEEE Transactions on Signal Processing, vol. 53, pp. 3791-3803, October 2005. 
[21] Y. Jiang, J. Li, and W. Hager, "Uniform channel decomposition for MIMO communications," IEEE Transactions on Signal Processing, vol. 53, pp. 42834294, November 2005.

[22] Y. Jiang, W. Hager, and J. Li, "The geometric mean decomposition," Linear Algebra and Its Applications, pp. 373-384, February 2005.

[23] H. Li, "A comparison of rateless codes for wireless communication systems," Master's thesis, Carleton University, 2007.

[24] J. Pearl, Probabilistic reasoning in intelligent systems. San Francisco, CA: Morgan Kaufmann Publishers Inc, 1998.

[25] R. M. Tanner, "A recursive approach to low complexity codes," IEEE Transactions on Information Theory, vol. IT-27, pp. 533-547, 1981.

[26] D. M. Mandelbaum, "An adaptive-feedback coding scheme using incremental redundancy," IEEE Transactions on Information Theory, vol. IT-20, pp. 388389, May 1974.

[27] E. Choi, S.-B. Suh, and J. Kim, "Rate-compatible puncturing for low-density parity-check codes with dual-diagonal parity structure," in IEEE 16th International Symposium on Personal, Indoor and Mobile Radio Communications (PIMRC), (Berlin, Germany), pp. 2642-2646, 2005.

[28] J. Ha, J. Kim, D. Klinc, and S. McLaughlin, "Rate-compatible punctured lowdensity parity-check codes with short block lengths," IEEE Transactions on Information Theory, vol. 52, pp. 728-738, February 2006. 


\section{Appendix A}

\section{UCD Properties and Algorithm}

\section{A.1 Reduced Complexity UCD Algorithm}

We present a reduced complexity method for computing the UCD algorithm that is given in [21]. The use of the reduced complexity algorithm means that less matrix operations are needed to find the UCD of a channel matrix. The computational complexity degree order remains three due to the use of SVD. The reduced complexity UCD algorithm is employed in the simulations that were used to test the performance of UCD in this thesis.

1. The first step in computing the UCD of a matrix is to compute the channel's SVD as specified in (2.17). The unitary pre-coder matrix is set to equal the right-hand unitary matrix of the SVD $\left(\boldsymbol{\Omega}_{1}=\mathrm{V}\right)$.

2. The second step is to use the WFA based upon (2.19) to find the power allocation for each subchannel. This sets the matrix components of $\Phi$.

3. Once we have the singular value matrix of the channel and the power allocation matrix, we compute their product (i.e. $\Sigma=(\Lambda \Phi)^{\frac{1}{2}}$ ).

4. Calculate the matrix $\tilde{\boldsymbol{\Sigma}}$ using (A.4) 
5. The GMD of the channel power matrix is obtained using(2.23). We then set the second pre-coder matrix to be the right-hand unitary matrix of the GMD $\left(\Omega_{2}=\mathbf{P}\right)$

6. Find the resulting pre-coded channel matrix before spatial filtering (i.e. $\Gamma=\mathbf{H F})$

7. Using (2.14) - (2.16) the MMSE filter for matrix $\Gamma$ is found.

\section{A.2 UCD SINR Properties}

In this appendix section some matrix properties given in [21] are presented. The matrix properties are necessary for obtaining the SINR properties of the UCD transceiver.

Property A.2.I: For any $N_{r} \times N_{t}$ matrix $\mathbf{B}$ with rank $K$ and singular values $\left\{\sigma_{1}, \sigma_{2}, \cdots, \sigma_{K}\right\}$ with augmented matrix

$$
\mathbf{A}=\left[\begin{array}{c}
\mathbf{B} \\
\sqrt{N_{0}} \mathbf{I}_{N_{t}}
\end{array}\right]
$$

have their singular values are related by

$$
\sigma_{A, i}=\sqrt{\sigma_{B, i}^{2}+N_{0}}
$$

and their unitary matrices of their singular value decompositions are related by

$$
\mathbf{U}_{A}=\left[\begin{array}{l}
\mathbf{U}_{B} \Lambda_{\mathbf{B}}^{\frac{1}{2}} \Lambda_{\mathbf{A}}^{\frac{-1}{2}} \\
\sqrt{N_{0}} \mathbf{V}_{B}^{+} \Lambda_{\mathbf{A}}^{\frac{-1}{2}}
\end{array}\right] \text { and } \mathbf{V}_{A}=\mathbf{V}_{B}
$$


This means that the SVD of $\mathbf{J}$ as defined in (2.17) is

$$
\mathbf{J}=\left[\begin{array}{c}
\mathrm{U} \Sigma \tilde{\Sigma}^{-1} \\
\sqrt{N_{0}} \tilde{\Sigma}
\end{array}\right] \tilde{\Sigma} \mathbf{I}_{K}
$$

where $\tilde{\Sigma}$ is an $L \times L$ diagonal matrix with the following property

$$
\tilde{\sigma}_{i}=\sqrt{\phi_{i} \lambda_{i}+N_{0}}
$$

Applying a GMD to $\tilde{\Sigma}$ we obtain

$$
\mathbf{J}=\left[\begin{array}{c}
\mathrm{U} \Sigma \tilde{\mathbf{\Sigma}}^{-1} \\
\sqrt{N_{0}} \tilde{\Sigma}
\end{array}\right] \mathbf{Q}_{J} \mathbf{R}_{J} \mathbf{P}_{J}^{+}
$$

The matrices to the left of $\mathbf{R}_{J}$ give part of the $\mathbf{Q}_{\Gamma_{a}}$ and $\Omega_{2}$ is determined as usual by setting it equal to the unitary matrix on the right-hand side of $\mathbf{R}_{J}$. The diagonal entries of $\mathbf{R}_{J}$ are equal to

$$
r_{J, i i}=\left(\prod_{j=1}^{K} \tilde{\sigma}_{j}\right)^{\frac{1}{K}}=\left(\prod_{j=1}^{K} \sqrt{\sigma_{j}^{2}+N_{0}}\right)^{\frac{1}{K}}
$$

Property 2.3.4.II: The diagonal of the augmented upper triangular matrix computed to get a QR-based MMSE filter using (2.14) - (2.16) has diagonal elements such that:

$$
r_{G a, i i}^{2}=\left(1+\rho_{i}\right) N_{0}
$$

These properties are useful in evaluating the capacity of the UCD transceiver. 


\section{Appendix B}

\section{RC/QC LDPC Codeword Puncturing}

\section{Technique}

The RC/QC LDPC code has a mother parity-check matrix $\mathbf{H}_{m c}$, which is the minimum rate parity-check matrix. The structure of this parity check matrix is such that

$$
\mathbf{H}_{m c}=\left[\begin{array}{ll}
\mathbf{H}_{m c}^{\prime} & \emptyset \\
\mathbf{H}_{e m c}^{\prime} & \mathbf{I}
\end{array}\right]
$$

where $\emptyset$ is an all zero matrix and $\mathbf{I}$ is an identity matrix. The parity check matrix can be viewed as a base parity check matrix $\mathbf{H}_{m c}^{\prime}$ followed by its extended version composed of $\mathbf{H}_{e m e}^{\prime}$ and $\mathbf{I}$. The parity bits that are encoded using the identity matrix have degree one and as a result should be punctured first, starting with the bottom $L_{b}$ rows of $\mathbf{H}_{m c}$ and moving upwards. This feature of the $\mathrm{QC} / \mathrm{RC}$ codes allows for simple codeword puncturing for rates between $R_{c}^{(\min )}$ and $R_{c}^{(m i d)}$; where $R_{c}^{(m i n)}=\frac{K_{c}}{N_{c}^{(m a x)}}$ and $R_{c}^{(m i d)}=\frac{K_{c}}{N_{c}^{(m i d)}}$; and $N_{c}^{(m i d)}$ is the row length of $\mathbf{H}_{m c}^{\prime}$. Puncturing is done in blocks of size $L_{b}$ so that the complexity of the decoding algorithm can be reduced. If the required rate is larger than $R_{c}^{(m i d)}$, the puncturing pattern is not so straight forward. We use a puncturing rule that follows the $\mathrm{k}$-SR node concept described in $[27,28]$. 
The message word size, of $K_{c}$ bits, determines the parameters $N_{c}^{(m a x)}, N_{c}^{(m i d)}, L_{b}$. It also determines whether the message should be extended. This may occur when the message length is not chosen to be of size compatible with those appropriate to the 3GPP2 parity check matrices. For the purpose of this thesis we always choose $K_{c}$ to be of appropriate size, removing the need for message word extension. The RC/QC LDPC codes of [15] require a parameter $L_{a}=2 L_{b}$. An extra parameter $k_{B}$ is specified such that $K_{c}=k_{B} L_{a}$, where $6 \leq k_{B} \leq 11$. In addition, $N_{c}^{m i d}=\left(k_{B}+\frac{K_{i}}{2}\right) L_{a}$. We use $k_{B}=6$ in simulations. The other system parameters, which are determined by the message word length, are specified as follows

$$
N_{c}^{(\max )}=n_{B} L_{a}=2 n_{B} L_{b}
$$

where $N_{c}^{(\max )}$ is the size of an unpunctured codeword, c. For $k_{B}=6: n_{B}=33$ and $K_{i}=12$. This means that the minimum rate, $R_{c}^{(m i n)}$, is $R_{c}^{(m i n)}=\frac{2}{11}$ and $R_{c}^{(m i d)}=\frac{1}{2}$. 